\title{
Visuo-vestibular interaction using rotatory chair and asynchronously moving visual target (normals, vestibular patients, whiplash patients)
}

Citation for published version (APA):

Konijnenberg, J. J. (1993). Visuo-vestibular interaction using rotatory chair and asynchronously moving visual target (normals, vestibular patients, whiplash patients). [Doctoral Thesis, Maastricht University]. Rijksuniversiteit Limburg. https://doi.org/10.26481/dis.19931208jk

Document status and date:

Published: 01/01/1993

DOI:

10.26481/dis.19931208jk

Document Version:

Publisher's PDF, also known as Version of record

Please check the document version of this publication:

- A submitted manuscript is the version of the article upon submission and before peer-review. There can be important differences between the submitted version and the official published version of record.

People interested in the research are advised to contact the author for the final version of the publication, or visit the DOI to the publisher's website.

- The final author version and the galley proof are versions of the publication after peer review.

- The final published version features the final layout of the paper including the volume, issue and page numbers.

Link to publication

\footnotetext{
General rights rights.

- You may freely distribute the URL identifying the publication in the public portal. please follow below link for the End User Agreement:

www.umlib.nl/taverne-license

Take down policy

If you believe that this document breaches copyright please contact us at:

repository@maastrichtuniversity.nl

providing details and we will investigate your claim.
}

Copyright and moral rights for the publications made accessible in the public portal are retained by the authors and/or other copyright owners and it is a condition of accessing publications that users recognise and abide by the legal requirements associated with these

- Users may download and print one copy of any publication from the public portal for the purpose of private study or research.

- You may not further distribute the material or use it for any profit-making activity or commercial gain

If the publication is distributed under the terms of Article $25 \mathrm{fa}$ of the Dutch Copyright Act, indicated by the "Taverne" license above, 
Visuo-vestibular interaction using rotatory chair and

asynchronously moving visual target (normals, vestibular patients, whiplash patients) 
- Jacobus Jannes Konijnenberg, Maastricht 1993

ISBN 90-9006755-8

Productie, lay-out en omslagontwerp: J.J. Konijnenberg Druk: Uniprint Maastricht 
Voor Lin, Dyan en Sander

Voor mijn ouders 
Het verschijnen van dit proefschrift werd mede mogelijk gemaakt door financiële steun van

- Stichting bevordering wetenschappelijk onderzoek $\mathrm{KNO}$-heelkunde, Maastricht

- Duphar Nederland B.V., producent van Betaserc $16^{\circ}$ 
Visuo-vestibular interaction using rotatory chair and asynchronously moving visual target (normals, vestibular patients, whiplash patients)

\section{PROEFSCHRIFT}

ter verkrijging van de graad van doctor aan de Rijksuniversiteit Limburg te Maastricht, op gezag van de Rector Magnificus, Prof. dr. H. Philipsen, volgens het besluit van het College van Dekanen, in het openbaar te verdedigen op woensdag 8 december 1993 om 14.00 uur

door

Jacobus Jannes Konijnenberg

geboren te Utrecht op 4 juni 1955 


\section{Promotor:}

Prof.Em. dr. E.H.M.A. Marres

\section{Co-promotor:}

Dr. H. Kingma

\section{Beoordelingscommissie:}

Prof. dr. J. Jolles, voorzitter

Dr. G.R. Barnes

Prof. dr. H. Collewijn

Prof. dr. F. Hendrikse

Prof. dr. J. Troost

Prof.Em. dr. E.H.M.A. Marres

Department of Ear, Nose and Throat medicine, State University of Limburg

Dr. H. Kingma

Deparment of Ear, Nose and Throat medicine, State University of Limburg

Dr. G.R. Barnes

Medical Research Council Human Movement \& Balance Unit, University of London

Prof. dr. H. Collewijn

Department of Physiology, Erasmus University of Rotterdam

Prof. dr. F. Hendrikse

Department of Ophtalmalogy, State University of Limburg

Prof. dr. J. Jolles, voorzitter

Department of Neuropsychology and Psychobiology, State University of Limburg

Prof. dr. J. Troost

Department of Neurology, State University of Limburg 


\section{Contents}

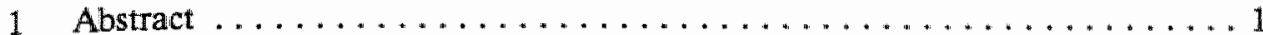

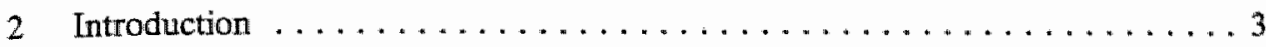

2.1 General introduction and aim of study $\ldots \ldots \ldots \ldots \ldots \ldots \ldots$

2.2 Introduction Visuo-Vestibular Interaction Test $\ldots \ldots \ldots \ldots \ldots \ldots$

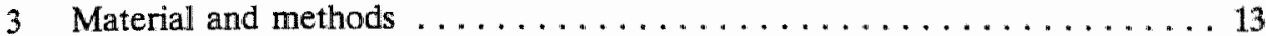

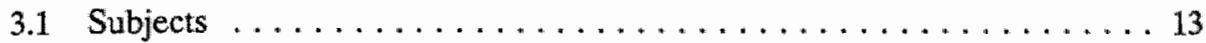

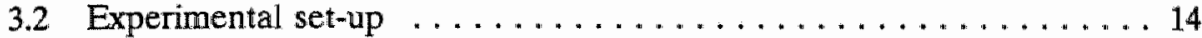

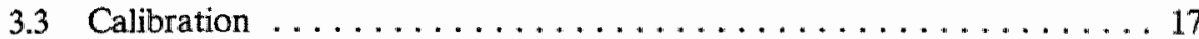

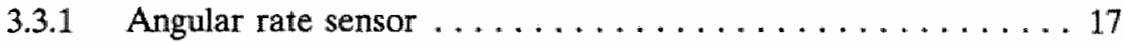

3.3 .2 Rotatory chair $\ldots \ldots \ldots \ldots \ldots \ldots \ldots \ldots \ldots \ldots \ldots \ldots$

3.3.3 Infra red reflection based detection of eye position $\ldots \ldots \ldots 19$

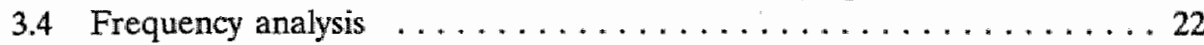

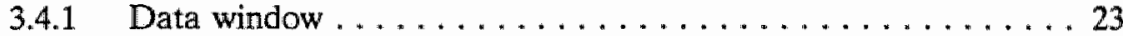

3.4 .2 Settings . . . . . . . . . . . . . . . . . . . 24

3.4 .3 Validation ........................... 26

4 Slow component characterization . . . . . . . . . . . . . . 29

4.1 One averaged velocity per slow component . . . . . . . . . . . 29

4.2 Replacement of fast components by using nonlinear filters . . . . . 30

4.2 .1 Median filter . . . . . . . . . . . . . . . . 31

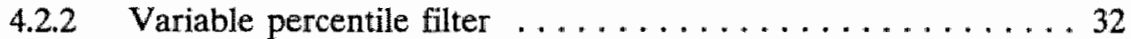

4.2 .3 Modal filter . . . . . . . . . . . . . . . . . . . . 34

4.2.4 Stimulus-ordered median filter . . . . . . . . . . . 35

4.2.5 Rank-trimmed moving-average filter for stimulus-ordered data . 37

5 Effect of asynchronous visual stimulation on the Vestibulo-Ocular Reflex

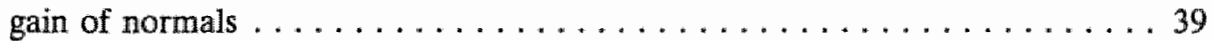

5.1 Abstract . . . . . . . . . . . . . . . . . . . . . . . 39

5.2 Introduction . . . . . . . . . . . . . . . . . . . 39

5.3 Material and methods . . . . . . . . . . . . . . 40

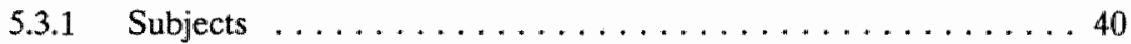

5.3 .2 Experimental set-up . . . . . . . . . . . . . . 41

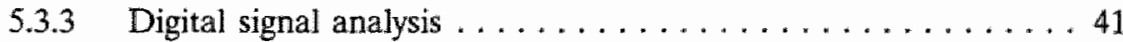

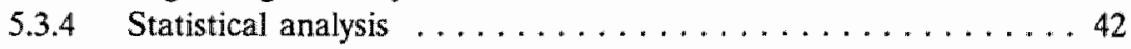

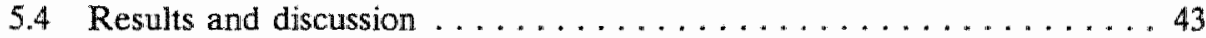

5.5 Appendix contrast ...................... 45 
6 Effect of asynchronous visual stimulation on the Vestibullo-Ocular Reflex

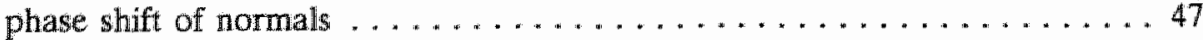

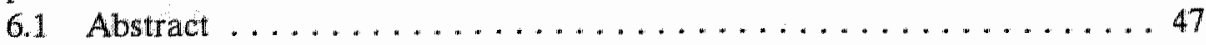

6.2 Introduction . . . . . . . . . . . . . . . . . . . . 47

63 Material and methods . . . . . . . . . . . . . . . . . 48

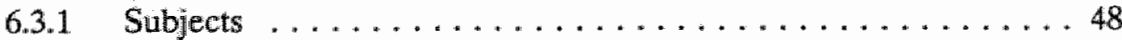

6.3 .2 Experimental set-up . . . . . . . . . . . . . . . . . . 49

6.3 .3 Digital signal analysis . . . . . . . . . . . . . . . 49

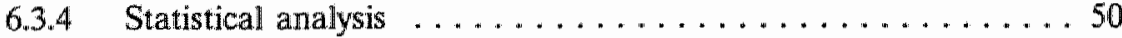

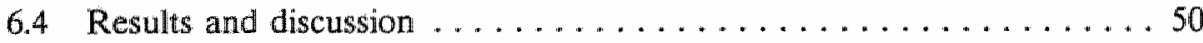

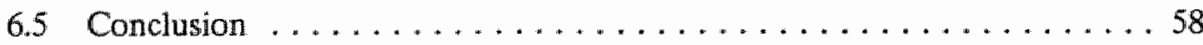

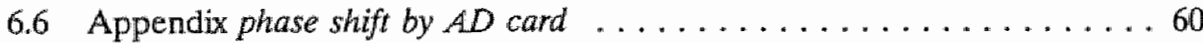

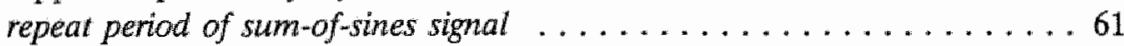

7 Effect of asynchronous visual stimulation on the Vestibulo-Ocular Reflex coherence of normals . . . . . . . . . . . . . . . . 65

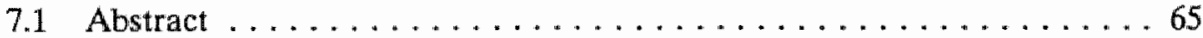

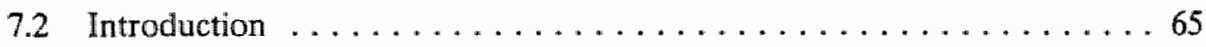

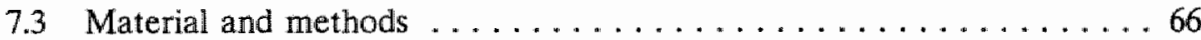

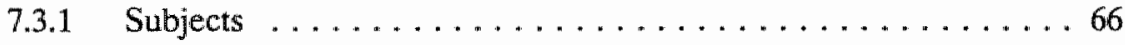

7.3 .2 Experimental set-up. . . . . . . . . . . . . . . 66

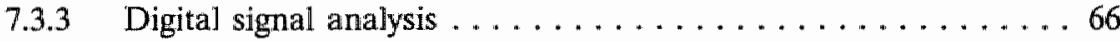

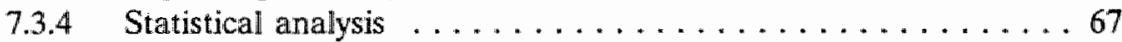

7.4 Results and discussion ...................... 67

8 Effect of asynchronous visual stimulation on the Vestibulo-Ocular Reflex gain of patients, compared to that of normals $\ldots \ldots \ldots \ldots \ldots \ldots 71$

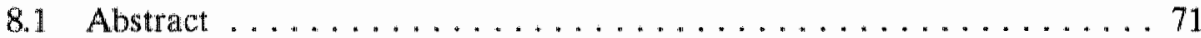

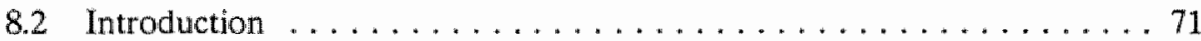

8.3 Material and methods . . . . . . . . . . . . . . . 72

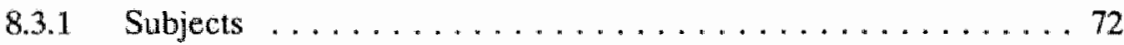

8.3.2 Experimental set-up ... . . . . . . . . . . . . 73

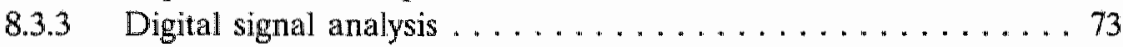

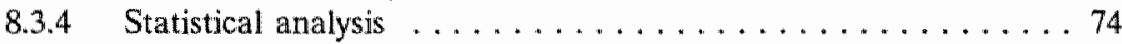

8.4 Results and discussion . . . . . . . . . . . . . . . . . . 74

8.5 Concllusion . . . . . . . . . . . . . . . . . . . . 85

8.6 Appendix Box-Cox transformation ................ 86 
9 Effect of asynchronous visual stimulation on the Vestibulo-Ocular Reflex phase shift of patients compared to that of normals . . . . . . . . 89

9.1 Abstract . . . . . . . . . . . . . . . . . . . . . . . . 89

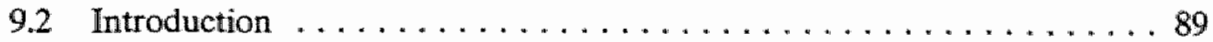

9.3 Material and methods . . . . . . . . . . . . . . . . . 90

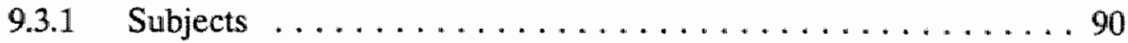

9.3 .2 Experimental set-up . . . . . . . . . . . . . . . . 90

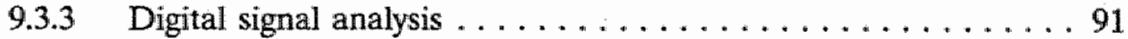

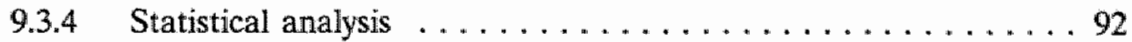

9.4 Results and discussion $\ldots \ldots \ldots \ldots \ldots \ldots \ldots \ldots \ldots \ldots \ldots \ldots . \ldots \ldots$

9.4.1 Visuo-vestibular interaction with an earth-fixed visual target . . 994

9.4.2 Visuo-vestibular interaction with an asynchronously moving

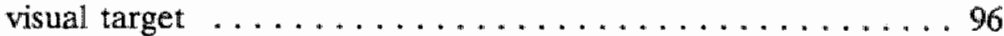

9.4.3 Individual contrasts of visuo-vestibular interaction with a visual target that is earth-fixed and with one that is moving

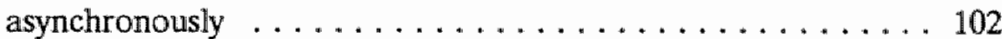

9.4 .4 Conclusions . . . . . . . . . . . . . . . . . 107

10 Effect of asynchronous visual stimulation on the Vestibulo-Ocular Reflex coherence of patients, compared to that of normals . . . . . . . 109 10.1 Abstract . . . . . . . . . . . . . . . . . . . . . . 109

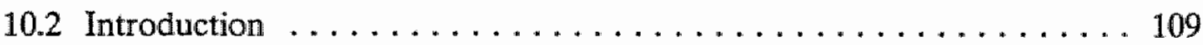

10.3 Material and methods . . . . . . . . . . . . . . . . 110

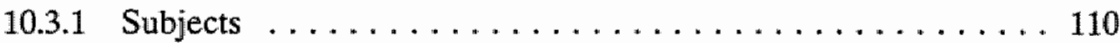

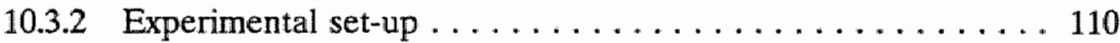

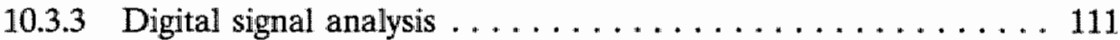

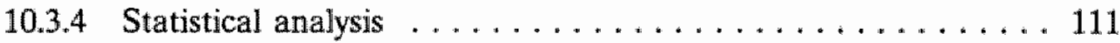

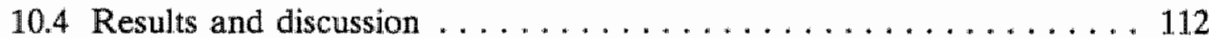

10.4.1 Visuo-vestibular interaction with an earth-fixed visual target . . 113

10.4.2 Visuo-vestibular interaction with an asynchronously moving

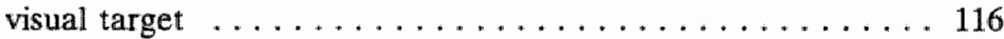

10.4.3 Individual contrasts of visuo-vestibular interaction with a visual target that is earth-fixed and with one that is moving

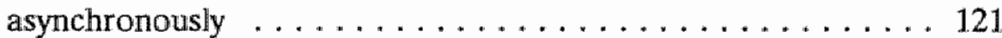

10.4 .4 Conclusions . . . . . . . . . . . . . . . . . . . 131 
11 Discriminating power of VVIT for type of pathology compared to those

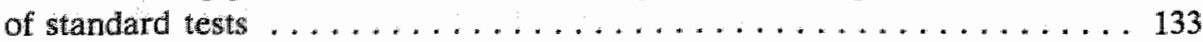

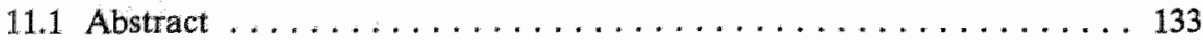

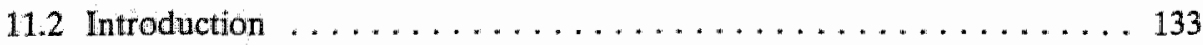

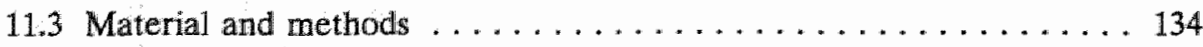

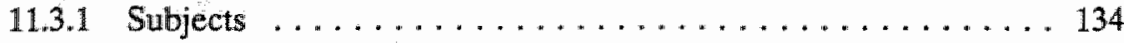

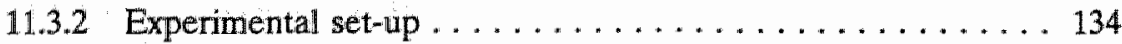

11.3 .3 Digital signal analysis . . . . . . . . . . . . . . 135

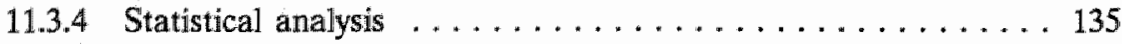

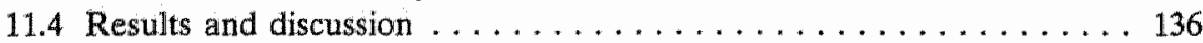

12 Discriminating power of VVIT for side of pathology, compared to that of

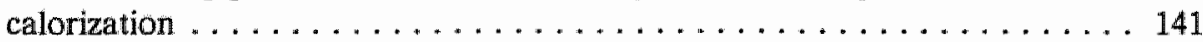

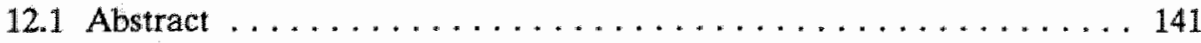

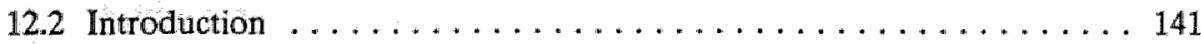

12.3 Material and methods . . . . . . . . . . . . . . . . . . 142

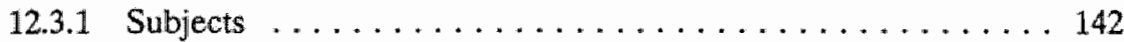

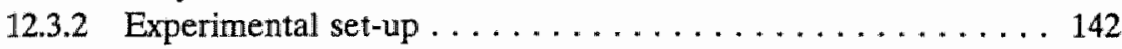

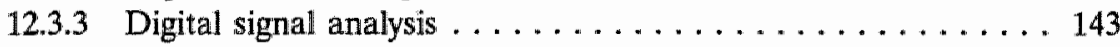

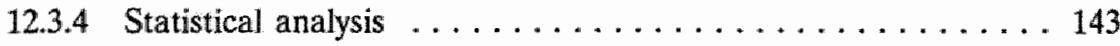

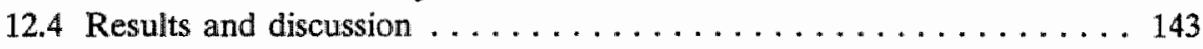

12.5 Appendix push-pull configuration $\ldots \ldots \ldots \ldots \ldots \ldots \ldots \ldots \ldots \ldots$

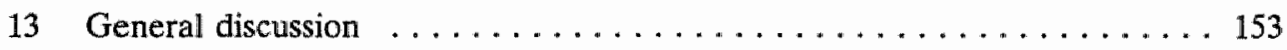

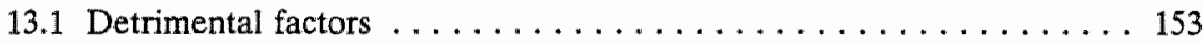

13.2 Group 3 might consist of 2 sub groups $\ldots \ldots \ldots \ldots \ldots \ldots \ldots \ldots \ldots$

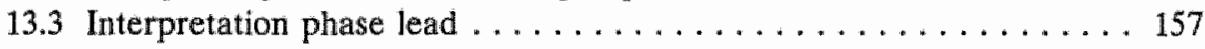

13.4 Separation of vestibular patients and normals by the Visuo-

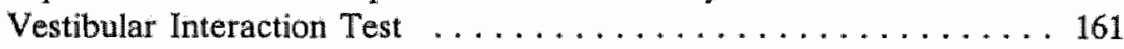

13.5 Conclusions . . . . . . . . . . . . . . . . . 164

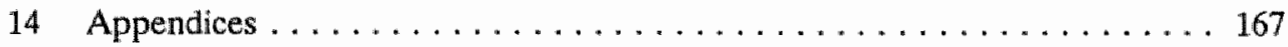

14.1 Sum-of-sines pseudo-random stimuli $\ldots \ldots \ldots \ldots \ldots \ldots \ldots \ldots \ldots . \ldots \ldots$

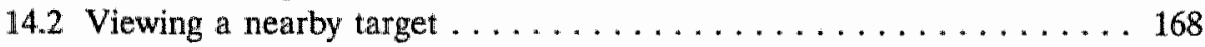

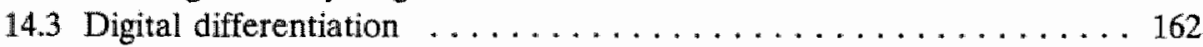

14.4 Multivariate analysis of variance (MANOVA) $\ldots \ldots \ldots \ldots \ldots 171$

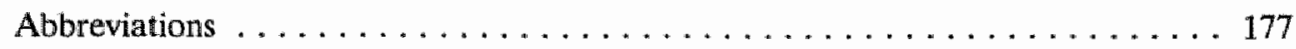

References . . . . . . . . . . . . . . . . . . . . . . . . . 179 


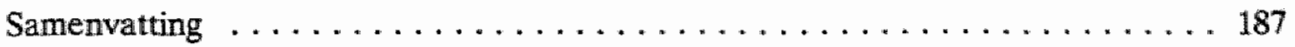

Dankwoord / Acknowledgements . . . . . . . . . . . . . . . . . . . . 189

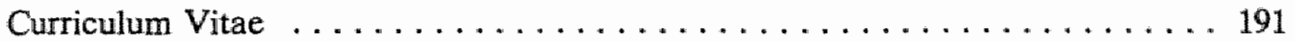




\section{Abstract.}

. vestibular function test (named Visuo-Vestibular Interaction Test: VVIT) is resented, in which a visual target may move asynchronously with a pseudo-randomly scillating rotatory chair.

s results allow better classification of the studied patients than the results of the sed standard tests (single-sine oscillation in the dark, calorization). Its specificity with sspect to normals only, is 1.00 for the six controls used, and its sensitivity is higher uan that of the used standard tests for Meniere patients, centrally poorly compenated peripheral vestibular patients, whiplash patients, and patients having benign aroxysmal positioning dizziness (BPPD), but not for centrally well compensated eripheral vestibular patients and patients for whom the causes of their complaints re unknown. Whiplash patients, Meniere patients, and BPPD patients are the groups or which the VVIT has the best combinations of sensitivity and specificity with espect to other patient groups as well as controls.

ix normals and 64 patients were examined, and of the latter, 51 remained for nalysis.

Jonsiderable effort has been put in finding and correcting measurement errors, which s described in some detail.

ignal processing included the use of rank filters to remove fast components and to ittenuate eye blink artefacts. The median filter was generalized into a "variable sercentile filter" to process nystagmoid eye signals. It was also used on stimulusrdered (and thus time-scrambled) signals to improve gain extraction by linear egression from pseudo-random signals without resorting to frequency analysis, hereby enabling to compare the gains for leftward and rightward movements.

ignal analysis was mainly done, however, by using frequency analysis. Gain, phase hift, and coherence were calculated in the frequency domain.

The results were statistically analysed by various forms of multiwariate analysis of rariance (MANOVA's), and by calculating sensitivity and specificity using standard, as vell as unbiased reference ranges. 


\section{Introduction.}

\section{General introduction and aim of study.}

rganisms detect their orientation with respect to their surroundings, and the agnitude and direction of their movements. Humans, as most vertebrates, do so in der to balance themselves and to avoid collisions. Much of the necessary inforation processing is done without making it available to the higher cortical areas, so lat we are little aware of it. Until, of course, the system does not function well and te consequences are met. For reasons given below, vestibularly function is studied a eye movements.

lwestigations of vestibularly and visually guided eye movements can be divided into vo fields. At the one hand, fundamental research aims to elucidate in what way the istems work, and at the other hand, medical diagnostics aims to assess in how far the istems work. Of course, neither field really can do without the other, but this ivision serves well to point out the scope of this thesis: medical diagnostics. We jught to develop a vestibular function test whose results of normals and patients rould generally agree with those from the commonly used tests, while correlating etter with the complaints, and which would have less variability. Our strategy was to ave the test stimuli mimic natural stimulation more closely, and to concentrate on nalyzing parameters having natural significance (performance parameters like gain, thase shift and coherence, as opposed to nystagmus-related parameters).

The vestibular system can be considered to be a complex system, because it receives is input from multiple sources, processes the information at diverse locations and ends its output to various targets (textbooks: Carpenter (1988), Howard (1986)). The nput comes from the vestibular end organs (semi-circular canals for detecting rotary novements, utriculus and sacculus for linear movements; all located in the middle ars), the eyes (providing visual information), proprioceptors (signaling the relative rositions and relative movements of the parts of the body), and miscellaneous eceptors like baroreceptors (signaling, e.g., the foot sole pressure, or the blood rressure in various parts of the body (Mittelstaed, 1991)). Processing and integration of information is done at various centers in the brain stem (see figures 2-1 and 2-2). inally, the resulting output is sent to the extra-ocular muscles (for gaze stabilization) ind to skeletal muscles (for balance and movement), thus completing several nechanical feedback loops, and some is also sent to higher areas in the cerebral ortex (for orientation and sensation).

When studying such a complex system, one would like to be able to measure input and output from each part separately. However, the vestibular end organs and the sentral nervous system are hard to reach, making it unethical with current techniques 


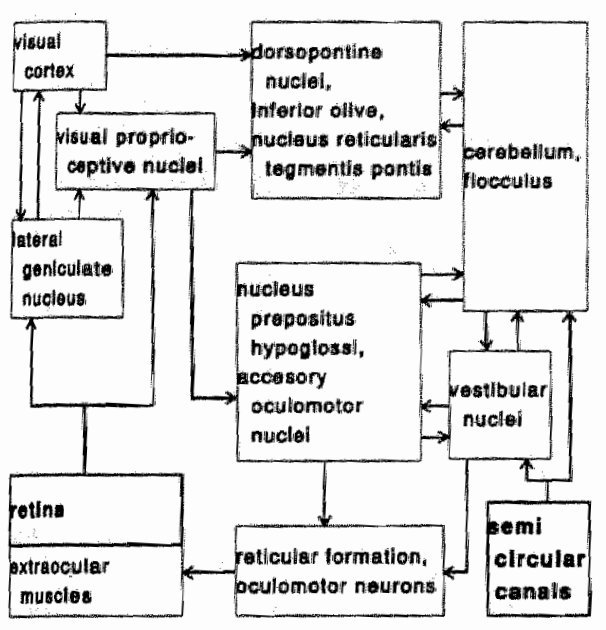

Figure 2-1. Main interactions for gaze bolding. Visual proproceptive nucdeil = pretectal auclei + accessiory optic system; accessory cocibmotor nuclei = interstitial, nuclei of Cajal + intershtial node of the medial fongitedinal las. ciculus + muelel of Darkschewitsch + nuclei of the posterfor commissure. (After Carpenter, 1988).



Figure 2-2 Appratimate locations in the brain and braimstem of some structures and muclei, relevant for gaze holding. Bilaterall enuties are projected on the shown mid. sagitual plane.

to do so in humans. Therefore, the behavior of larger units (i.c., ocular and postural reflexes, and sensations) is investigated. This study concerns the horizontal eye movements resulting from integrating visual and vestibular information, and it tries to extract from them information about vestibular functioning. The eye movements are restricted to horizontal ones, the visual information is restricted to a small target moving along a tangent, horizontal line (thus stimulating the fovea only, at almost one vergence angle), and the vestibular stimulation is restricted to passive rotary wholebody movements around the vertical axis with subjects sitting upright.

Two new' methods are introduced: vestibular stimulation with asynchronous visual target movement, and analysis using rank filters to remove fast components from the eye movement signal.

To validate the new method of analysis, it had first to be used on data from the stan dard tests. And to validate the new test, its results had to be compared with those o

\footnotetext{
1 After cowpteting this thesis, it was found that Baraes ef al. (1985) had reported on asynchronous visual and vestibul stumulation, and that Engeiken \& Stevens (1990) had published over using order statistic filters tor nystagmus anahyse.
} 
the standard tests. The first made it necessary, and the second desirable, to generalize the median filter into a variable percentile filter and to introduce a modal filter, in order to reject not only random spikes, but also the correlated nystagmus fast components.

\subsection{Introduction Visuo-Vestibular Interaction Test.}

Of the vestibulo-ocular reflexes (VOR's) the translational one (tVOR) will be ignored here, and of the remaining rotational ones (rVOR's) only that around an earthvertical axis ( $z$ ) is considered. So the prefix $r_{z}$ is dropped and it is to be understood that "VOR" stands for the horizontal rotational vestibulo-ocular reflex.

The frequency range in which the VOR functions, can be divided in low (f $<1 \mathrm{~Hz}$ ), where the visual system dominates (Benson \& Barnes, 1978; Barnes et al, 1978), intermediate $(1<\mathrm{f}<3 \mathrm{~Hz})$, where the visual and vestibular systems interact, and high ( $\mathrm{f}>3 \mathrm{~Hz}$ ), where the vestibular system dominates (Hydên et al., 1982).

In order to study the "pure" VOR in the low and intermediate frequency ranges, no visual information about his movements should be available to the subject. Usually, this is accomplished by rotating the subject in the dark, although Barr et al. (1976) have shown that opaque contact lenses work equally well, as did Goebel et al. (1991) with a featureless blank field (for the intermediate frequencies).

It seems, however, that the subject is moving his eyes according to his mental representation of the surroundings, rather than according to what he actually sees (Steinbach, 1976; Barnes \& Asselman, 1991; see also Bahill et al, 1980, referencing Stark et al,, 1962: "predictor", Dallas \& Jones, 1963: "long term learning process", Yasui \& Young, 1975: "percept tracker", McDonald and Bahil, 1980: "adaptive controller"; Bloomberg et al., 1991a, 1991b). In absence of visual information, different subjects can have different mental representations, introducing considerable variability (Barr et al., 1976). By giving specific instructions (either "imagine a stationary dot on the wall" or "imagine a dot moving with the chair"), the overall variability can be reduced, leaving only the extra variation due to differences in the capabilities to construct mental models.

The same instructions were found to influence results only in case of sinusoidal stimulation in the low and intermediate frequency ranges, but not during high frequency testing (Larsby et al, 1984). There is some disagreement with respect to this voluntary VOR modification during pseudo-random vestibular stimulation. Using a $0.2-4.5 \mathrm{~Hz}$ white-noise pseudo-random rotation, Larsby et al. (1984) found no effect of these instructions, while McKinley \& Peterson (1985) and Furst et al. (1987), using sum-of-sines pseudo-random stimulation in $0.25-1.9 \mathrm{~Hz}$ and $0.05-0.35 \mathrm{~Hz}$ frequency 
ranges, respectively, found that imagining a target is equally effective during single: sine and during pseudo-random stimulation. It has been suggested that the sum-of sines pseudorandom stimulation still allows prediction and thus permit the construc tion of mental models. While for purely visual stimuli, Barnes et al. (1987) have shown that the predictability of a smooth pursuit (SP) stimulus does not depend on its complexity, but on its highest-frequency component, for the VOR in the dar] Barnes \& Eason (1987) found no such an effect of the highest frequency when th subjects were discriminating auditory pitches and small effects when they wer imagining a head-fixed target.

In the low and intermediate frequency range, the VOR response can approximatel be characterized by the transfer function (Honrubia et al, 1982):

$$
\begin{aligned}
& H(s)=\frac{\dot{e}}{h}=\frac{s K}{1+s T} \\
& \text { e eye velocity } \\
& \text { h head velocity } \\
& \text { H transfer function } \\
& \text { K sensitivity constant } \\
& \text { s Laplace variable } \\
& \text { T dominant time constant }
\end{aligned}
$$

which has for sinusoidal stimuli a gain of:

$$
\begin{aligned}
& \operatorname{gain}(\omega)=|H(\omega)|=\frac{\omega K}{\sqrt{1+\omega^{2} T^{2}}} \\
& \text { H transfer function } \\
& \text { K sensitivity constant } \\
& \text { T dominant time constant } \\
& \omega \text { circular frequency }
\end{aligned}
$$

and so at low frequencies ( $\mathrm{f}<0.02 \mathrm{~Hz}$ ) the gain is proportional to stimulus freque and to the sensitivity constant (Baloh et al, 1984):

$$
\begin{aligned}
& g-\omega K \quad\left(\omega^{2} T^{2}<1\right) \\
& \begin{array}{l}
\text { g velocity gain } \\
\text { K. sensitivity constant } \\
\text { circular frequency }
\end{array}
\end{aligned}
$$


while at intermediate frequencies ( $\mathrm{f}>0.1 \mathrm{~Hz}$ ) the gain is independent of frequency, proportional to the sensitivity constant and inversely proportional to the dominant time constant:

$$
\begin{aligned}
& g-\frac{K}{T} \quad\left(\omega^{2} T^{2}>1\right) \\
& \text { g velocity gain } \\
& \mathrm{K} \text { sensitivity constant } \\
& \mathrm{T} \text { dominant time constant }
\end{aligned}
$$

In case of a diminished vestibular sensitivity, the system can adapt by decreasing the dominant time constant (ie., by shortening the memory of the neural integrator). This is known as the process of central adaptation. The gain remains then unaffected by the loss of sensitivity for the middle, but not for the low frequency range. This latter, though, will give little problems for the patient, as he will rely more heavily on his visual system which functions best at low frequencies.

From the above it will be clear, that low frequency testing of the VOR only allows detecting vestibular dysfunctioning when done without providing visual information. And it does so largely independent from the degree of central compensation. Test results can correlate poorly with the patients' complaints, however. Tests may detect abnormal overall gains, abnormal labyrinthine directional preponderances (i.e., asymmetries in gain for leftward and rightward rotations), and/or abnormal phase shifts for certain frequencies. Complaints may correlate with only some, or only some combinations, of these. Wolfe et al. (1982), e.g., concluded that decrements in phase shifts at very low frequencies correlate with the degree of peripheral impairment, and that the asymmetry correlates with subjective dizziness. Precht \& Dieringer (1985) stress that adaptation of the gain after unilateral peripheral loss is controlled independently from restoration of symmetry, and that the control sites are different. Which is not to say, however, that gain can not be controlled by the same mechanism that controls symmetry. Galiana (1985) argues that because the generally accepted scheme for commissural inhibition allows positive feedback, this can influence not only symmetry, but overall gain as well. In all, one complaint may result from multiple causes, and conversely, one cause may result in multiple complaints.

Complaints can be divided in two frequency-related categories.

Vertigo is thought to result from sensory conflicts (Claremont, 1931) and from conflicts between sensory information and centrally stored information (Reason, 1970, 1978). Vertigo originating from some pathology, then, might mostly occur in a frequency range where several systems produce relevant output. Because the visual 
ranges, respectively, found that imagining a target is equally effective during singlesine and during pseudo-random stimulation. It has been suggested that the sum-ofsines pseudo-random stimulation still allows prediction and thus permit the construction of mental models. While for purely visual stimuli, Barnes et al. (1987) have shown that the predictability of a smooth pursuit (SP) stimulus does not depend on its complexity, but on its highest-frequency component, for the VOR in the dark Barnes \& Eason (1987) found no such an effect of the highest frequency when the subjects were discriminating auditory pitches and small effects when they were imagining a head-fixed target.

In the low and intermediate frequency range, the VOR response can approximately be characterized by the transfer function (Honrubia et al, 1982):

$$
\begin{aligned}
& H(s)=\frac{\dot{e}}{\dot{h}}=\frac{s K}{1+s T} \\
& \text { e. eye velocity } \\
& \text { b head velocity } \\
& \text { H transfer function } \\
& \text { K sensitivity constamt } \\
& \text { s Laplace variable } \\
& \text { T dominant time constant }
\end{aligned}
$$

which has for sinusoidal stimuli a gain of:

$$
\begin{gathered}
\operatorname{gain}(\omega)=|H(\omega)|=\frac{\omega K}{\sqrt{1+\omega^{2} T^{2}}} \\
\mathbf{H} \quad \begin{array}{ll}
\text { transfer function } \\
\mathrm{K} & \text { sensitivity constant } \\
\mathrm{T} & \text { dominant time constant } \\
\omega & \text { circular frequency }
\end{array}
\end{gathered}
$$

and so at low frequencies ( $\mathrm{f}<0.02 \mathrm{~Hz}$ ) the gain is proportional to stimulus frequency and to the sensitivity constant (Baloh et al, 1984 ${ }^{2}$ ):

$$
g \sim \omega K \quad\left(\omega^{2} T^{2}<1\right)
$$

$$
\begin{array}{ll}
\mathrm{g} & \text { velocity gain } \\
\mathrm{K} & \text { sensitivity constant } \\
\omega & \text { circular frequency }
\end{array}
$$

\footnotetext{
Be aware of the change from gye velocity versus head acceteration to eye welocity versus head velocity in this reference
} 
while at intermediate frequencies ( $f>0.1 \mathrm{~Hz}$ ) the gain is independent of frequency, proportional to the sensitivity constant and inversely proportional to the dominant time constant:

$$
\begin{aligned}
& g-\frac{K}{T} \quad\left(\omega^{2} T^{2}>1\right) \\
& \begin{array}{ll}
\text { g velocity gain } \\
\mathrm{K} & \text { sensitivity constant }
\end{array} \\
& \text { T dominant time constant }
\end{aligned}
$$

In case of a diminished vestibular sensitivity, the system can adapt by decreasing the dominant time constant (i.e., by shortening the memory of the neural integrator). This is known as the process of central adaptation. The gain remains then unaffected by the loss of sensitivity for the middle, but not for the low frequency range. This latter, though, will give little problems for the patient, as he will rely more heavily on his visual system which functions best at low frequencies.

From the above it will be clear, that low frequency testing of the VOR only allows detecting vestibular dysfunctioning when done without providing visual information. And it does so largely independent from the degree of central compensation. Test results can correlate poorly with the patients' complaints, however. Tests may detect abnormal overall gains, abnormal labyrinthine directional preponderances (i.e., asymmetries in gain for leftward and rightward rotations), and/or abnormal phase shifts for certain frequencies. Complaints may correlate with only some, or only some combinations, of these. Wolfe et al. (1982), e.g., concluded that decrements in phase shifts at very low frequencies correlate with the degree of peripheral impairment, and that the asymmetry correlates with subjective dizziness. Precht \& Dieringer (1985) stress that adaptation of the gain after unilateral peripheral loss is controlled independently from restoration of symmetry, and that the control sites are different. Which is not to say, however, that gain can not be controlled by the same mechanism that controls symmetry. Galiana (1985) argues that because the generally accepted scheme for commissural inhibition allows positive feedback, this can influence not only symmetry, but overall gain as well. In all, one complaint may result from multiple causes, and conversely, one cause may result in multiple complaints. Complaints can be divided in two frequency-related categories.

Vertigo is thought to result from sensory conflicts (Claremont, 1931) and from conflicts between sensory information and centrally stored information (Reason, 1970, 1978). Vertigo originating from some pathology, then, might mostly occur in a frequency range where several systems produce relevant output. Because the visual 
system and the semicircular canal system (the otolith system is not considered here) both function in the middle frequency range, here conflicts could arise when one of the systems dysfunctions. After some time the central system adapts, presumably when it learns which one of the conflicting information flows is correct. We therefore used stimuli in the middle frequency range in order to study pathological vertigo.

Dynamic postural instability and oscillopsia, at the other hand, might mostly manifest themselves at high frequencies, where the visual system can not take over. At these frequencies, howewer, many mechanical problems hamper testing and so those complaints are not considered here.

Traditionally, VOR research, when being done in the dark, concentrates on ewoking nystagmus, and on suppression or enhancement of the VOR when studying visuovestibular interaction. But for test results to correlate best with complaints, we felt that test conditions should mimic natural conditions as close as possible (which, after all, provoke those complaints!).

Because the VOR has evolved to stabilize retinal images, the test obviously had to incorporate a visual target. In general, this means that only non-nystagmoid eye movements are relevant. For we analyze the signals assuming velocity control, which actually tries to stabilize the overall retinal image. If some threshold is surpassed, as with large-angle stimulation (vestibularly, as well as optokinetically), then a new fixation point is chosen by generating a saccade. Under natural conditions, however, one specific target has to stay centered on the fovea. This kind of position control can also be accomplished by velocity control, as long as no nystagmus occurs.

Although the VOR is produced by a feed-forward system, which acts independently from visual input, visual feedback is used to recalibrate its parameters. Recalibration can take place within minutes (Collewijn et al, 1983) and so might alter responses during testing. So the system parameters are well-defined when a visual target is present, but are unknown when testing in the dark. Mostly it is assumed that the SP overshadows the "pure" VOR and that little useful information about the vestibular system can be retrieved from the results of steady-state sinusoidal rotation with an earth-fixed visual stimulus. However, the warning to researchers of the SP system that "Whenever there is a discrepancy between the performance of pursuit and VOR cancellation, it is necessary to check that the VOR itself is functioning normally." (Leigh \& Zee, 1983, p 80), can be turned around. That discrepancy could be a measure of VOR dysfunctioning, when SP is normal.

Because it is becoming increasingly clear that the brain is using opportunistic strategies to optimize performance, instead of simply summing responses of linear systems, we doubt whether VOR cancellation (VOR with a head-fixed visual target) 
equals visuo-vestibular interaction (VVI). Several authors (e.g., Ödkvist et al, 1988) have reported that visual suppression of the VOR is done by a system different from the SP system. And because he found that the latency for cancellation is shorter than that for the visual system, Cullen et al. (1991) concluded it to be a non-visual mechanism. This makes the term "VOR cancellation" preferable to "visual suppression of the VOR". However, other results are also reported. Magenes et al. (1990) concluded from the visual suppression studies that the SP system probably plays the major role. Although this particular study was not discussed by Cullen et al, they explained the observed decline of non-visual VOR cancellation efficacy with increasing frequency and peak acceleration as an adaptive constraint. In this way the VOR responding to slow head movements due to gaze pursuit can be cancelled, while the VOR to quick ones due to walking, remains active. Magenes et al. had studied visually induced suppression of post-rotational nystagmus, which could mean that the stimuli were outside the physiological range of the VOR self-cancellation. Later, Huebner et al. (1992) concluded from their latency studies that "... an internal SP signal is the primary means by which the VOR is cancelled during passive combined eye-head tracking..." ( $P$ 1790), but also that "During passive rotation of subjects in a vestibular chair, however, a VOR self-cancellation mechanism might assume more importance." (p 1791). A remarkable result was reported by Bock (1982), who found no interaction at all between SP and VOR. Below $0.8 \mathrm{~Hz}$ the slow component of eye movement was in phase with the chair-fixed visual target (as expected for the SP), above $1.5 \mathrm{~Hz}$ in counter phase (as expected for the VOR), and at intermediate frequencies it flipped between these two states, with the time spent in the VOR state proportional to the stimulus frequency. The gain when in the SP state dropped linearly with frequency from 0.97 to 0.1 and when in the VOR state was 0.42 , independent from frequency. In general, VVI may consist of all mechanism, i.e., VOR, non-visual cancellation of the VOR (by different mechanisms during pursuit and during saccade generation (Laurutis \& Robinson, 1986)), opto-kinetic reflex (OKR) (Honrubia et al., 1992), and SP. Whether the VOR is cancelled, and if so, to what extent and by what mechanism, seems to depend on the context. When only cancellation is required, the SP does not need to interact with the VOR: switching-on self-cancellation conceivably can be triggered by any stimulus. Therefore, we use a visual target that moves independently from and asynchronously with the chair, thus forcing such an interaction.

In order to diminish mental influences, pseudo-random stimulation is used. Originally, we wanted to subtract the results of a SP from those of a visuo-vestibular interaction in order to obtain the vestibular contribution. But pseudo-random stimulation was not possible in our laboratory at that time; software limitations restricted us to sum-ofsines pseudo-randomness for vestibular stimulation and the limited spatial resolution of the screen to single-sine visual stimuli. Single-sine SP allows complete prediction, however, and so we opted for using a sum-of-sines visuo-vestibular interaction with an 
earth-fixed target (eftVVI, ie., a visually enhanced VOR) as a base line. By choosing the frequencies and amplitudes in such a way, that the peak velocity is around the upper limit of the SP system (circa $30 \%$; Honrubia et al., 1968), even the least responsive vestibular patient cam compensate chair movements allmost perfectly by visually tracking the apparent target movements. During the visuo-vestibular interaction with an asynchronously moving target (mtVVI), however, only a normal subject can move his eyes well. For his vestibular system compensates the chair movements sufficieritly, so that the SP system has to deal with the actual target movement only. Without vestibular gaze stabilization, the apparent target velocity exceeds the SP system's upper limit, and therefore we expect the vestibular patients to perform worse. So we ended up with the peculiar situation, that we subtract the responses to only chair movements from the responses to combined target and chair movements (leaving us with the visual contribution). We hypothesize that this can be interpreted as indicating the westibular functioning. The information about vestibular functioning is then assumed to be contained by the ratio of smooth and saccadic eye movements (which sum up to a gain of unity). By filtering the fast components out of the response signal, the smooth part remains, and it is for this part that the abovementioned arguments might hold.

This verbal account can be illustrated with the following models found in the literature.

A very general model of visuo-vestibular interaction without voluntary head movements is given in fig. 2-3. If the complex response of the vestibular part $G_{\mathrm{v}}$ is impaired (lower gain and/or greater phase shift), then without an increased visual feedback the retinal position error $\epsilon$ will grow. If possible, the retinal slip (velocity error) will first cause the gaze holding part to move the eyes. Whether it is possible for the visual system to do so smoothly, depends on how heavily it is already loaded by the movement of the visual target. Any remaining retinal position error will cause the gaze shifting part to catch up by generating saccades.

Simplifying this model by leaving out the gaze shifting part, by lumping together the vestibular response, the behavior of the extra-ocular eye muscles, and eye ball dynamics in $G$ at the one hand, and visual processing and the eye plant characteristics in $F$ at the other, and at the same time adding more details from the gaze holding part, the model depicted in fig. 2-4 is obtained.

This figure shows two limitations of the visual smooth pursuit system, compared to the vestibulo-ocular reflex arc. First, there is a delay (as a consequence of the higher number of synapses, necessary to do higher-level information processing). And second, the gain of the feedback loop is influenced in a saturating way by the magnitude of the velocity error. The first limitation puts an upper bound of about $2 \mathrm{~Hz}$ to the frequencies that can be optimally processed by the smooth pursuit 


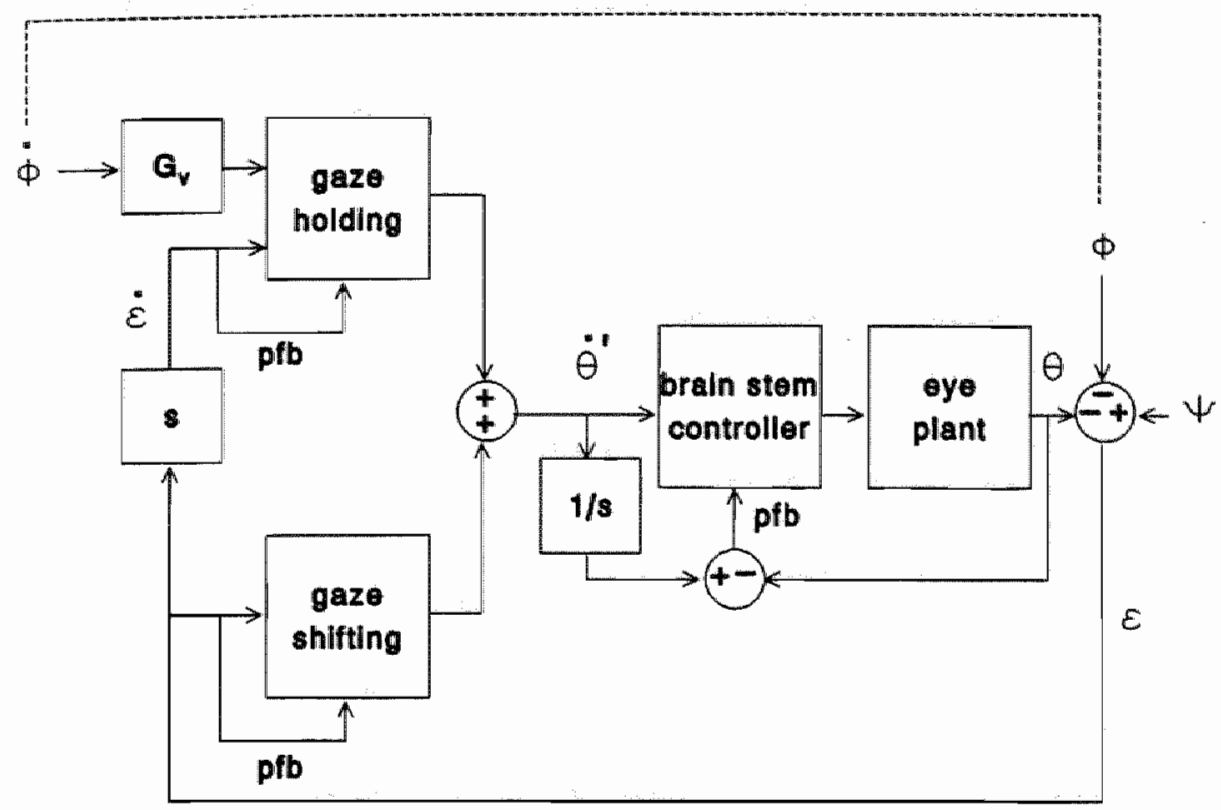

Fip. 2-3. General model of visto-vestibular inteltaction without active head mokements. $\dot{\phi}=$ head velocity; $\phi=$ head position;

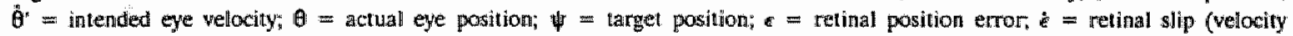
error); $\mathrm{G}_{\mathrm{V}}=$ transfer function of the vestibular system; $s=$ Laplace variable (single $\mathrm{s}$ is differentiation, $1 / \mathrm{s}$ is integration); $\mathrm{plb}=$ parametric feedback brain stem controler includes neural integrator. (After Carpenter, 1988, $\mathrm{p}$ 376).

system. This is here of no consequence, as our highest frequency is only $1.15 \mathrm{~Hz}$. The second limitation, however, is supposed to be pivottal to the Visuo-Vestibular Interaction Test. We assume that if we fully use the smooth pursuit capability by presenting a moving target with its speed at the upper limit, any deficiency of the vestibular part can only be compensated by the gaze shifting part through saccades, not by the gaze holding part through smooth pursuit movements. Further, we presume that by moving the chair and the target asynchronously, we will be able to study in the frequency domain the effects of the visual and vestibular system independently from each other.

It is assumed here, that vestibular stimulation will result in only smooth eye movements, not in saccadic ones. Bloomberg et al. (1991a, 1991b), however, reported that 


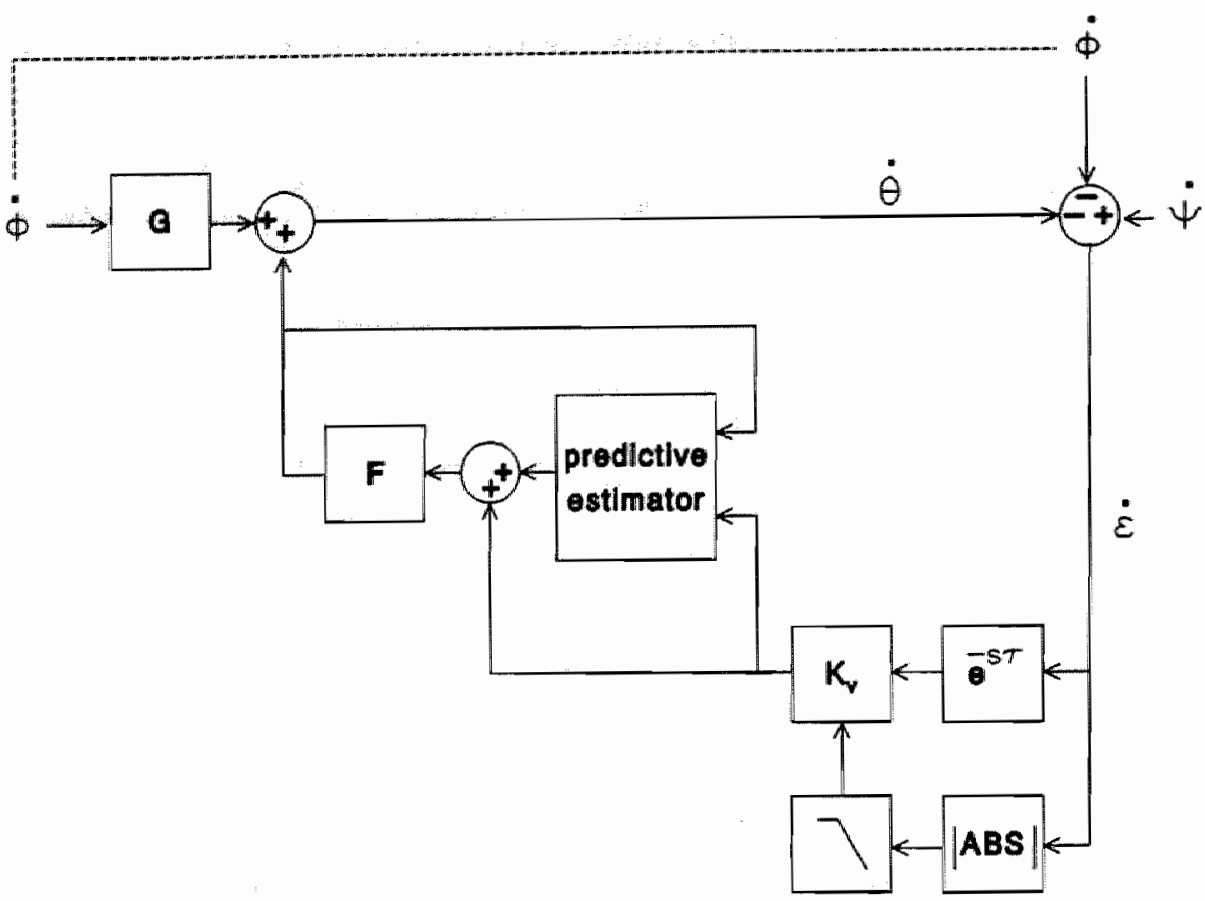

Firg. 2-4. A (still general) detail of a modiel for visuo-vestibular interaction without active heac movements. $\dot{\phi}=$ head velocity; 0 eye velocity, $*$ retinal slip (velocity error); $\pi=$ wisual processing dellay; $K_{v}=$ gain of visuall feedback loop (value is determined by the magnihude of the veloeiry error in a nonlinear way): $F=$ a lumped parameler representation of visual system, extranocular muscles, and ball dymamic behavior, $G=$ a lumped parameter representation of semicircular canals, central westibulat processing, waracular muscles, and eye ball dynamic behawior. (After Barnes, 1993).

subjects who tried to Jook in the dark towards a remembered position while being rotated, used both smooth and saccadic compensatory eye movements in a synergistic way. As long as their ratio remains constant, this would only diminish the VVIT's sensitivity, but it would not bias the results.

The sines constituting the pseudo-random stimulus are so chosen that they are not only harmonically unrelated to each other (in order to increase the period length of the sum; Stark et al, 1962), but that they are also unrelated to their pairwise sums and differences (to enable the calculation of a coherence function; see appendix 14.1; Maki, 1986, 1988). 


\section{Material and methods}

\subsection{Subjects}

Fifty-one vestibular patients were examined with the visuo-vestibular interaction test (VVTT), as well as with standard tests. An experienced vestibulologist (dr. H. Kingma), routinely diagnosed the patients according to their anamneses and their performance at standard tests, prior to being informed about their VVIT outcomes. Resulting diagnoses and numbers of patients per group, together with gender imbalances and median ages, are given in table 3-1. Age distributions are given in more detail in figure 3-1 as box-and-whisker plots. Also six controls without any history of vestibular complaints, were examined.

Apart from the whiplash patients, table 3-1 probably gives a fairly typical cross section of our patient population, as only those for whom anamnesis strongly suggested other causes than horizontal semicircular canal functioning and related central processing, were excluded. In a parallel multi-center study, recent whiplash victims were followed in time, and some were also tested using the VVIT. It was noted that they scored very differently from the others, and so more of them were refered to us by dr. J. Patijn from the Foundation for Manual Therapy in Eindhoven. Because only women were included in that whiplash study, this caused the gender imbalance for this group. No explanations are available for the severe imbalances for

Table 3-1. Group code number, diegnosis and descriptors of the srudied groups. Analyzed groups are printed in bold.

\begin{tabular}{|c|c|c|c|}
\hline diagnosis & $\begin{array}{l}\text { number of } \\
\text { subjects }\end{array}$ & $\begin{array}{l}\operatorname{sex} \\
\text { imbalance }(*)\end{array}$ & median age \\
\hline 0. consituls & 6 & 0.0 & 30 \\
\hline 1.. initial stages of Meniere's syndmome & 4 & o.s & 32 \\
\hline 2 peripheral westibular dysfunction, well compensated & 8 & $\mathbf{0 3}$ & 56 \\
\hline 3. peripheral westibular dystumetion, poorty compensatod & 13 & -0.1 & 45 \\
\hline 4. Whiplussh traumm & 10 & 0.2 & 45 \\
\hline 5. hyperventilation & 2 & 0.0 & 41 \\
\hline 6. benign paroxysanal positionings dizziness & 3 & -03 & 32 \\
\hline 7. vestibular complaints from unknown causes & 4 & 0.0 & 46 \\
\hline 8. excessive central inhibition & 1 & - & 37 \\
\hline 9. cerebelllar dysfunctioning & 1 & - & 26 \\
\hline 10. impaired adaptation & 2 & sete & 39 \\
\hline 11. impaired thabituation & 1 & - & 47 \\
\hline 12. multiple dystunctionings & 2 & $\ldots$ & 67 \\
\hline
\end{tabular}




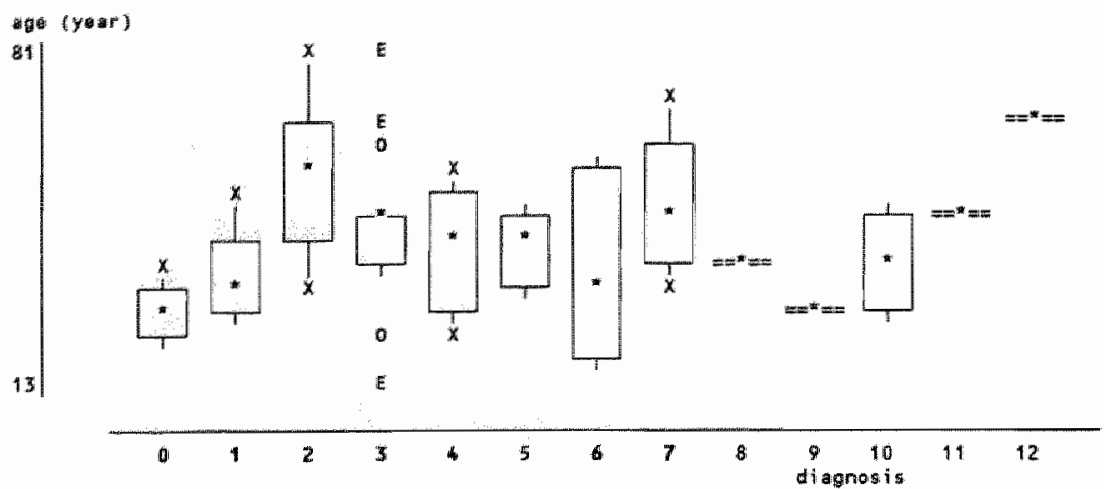

Irigure 3-1. Box plots showting age distributions of the diagnostically defined groups. Diagnostic codes of groups containing three or more subjects are analyzed and are printed in bold (see table 1 for explanation of the diagnostic codes). Vertical places may deviate from grtuall: wallues, because the program SPSS generated this graph as an ASCI character plot. - median (50\%), lower/upper quartile $(25 \% / 75 \%), \mathrm{X}=$ low/high (less than one interquartile range from lower/upper quartile), $0=$ outlier (any value more than one interguartile range, but less than one-and-analf, from lover/upper quarile), $E$ = extreme (any value more than one-and-a-half interquartîle rangesi from lower/upper quartilej.

the patients having initial stages of Meniere's syndrome, for those having well compensated, peripheral vestibular dysfunctioning, and for those having benign paroxysmal positionings vertigo. They probably deviate from the average by change, due to the extremely small sample sizes. The lower median age from the controls stems from recruiting them from our students. Figure 3-1 shows that the age distribution of group 3 (partly compensated, peripheral vestibular dysfunction) is markedly non-Normal: it has one of the smallest inter-quartile distances and at the same time the biggest range (making it leptokurtic).

None of the subjects had problems with visually guided eye movements. All subjects, even those usely wearing glasses, could locate the visual target without any optical correction, and were therefore tested that way.

\subsection{The experimental set-up.}

The experimental set-up was composed of an IBM compatible PC-286/287, a visual stimulator (AMIGA 500C (Commodore, Germany) with $73 \mathrm{~cm}$ TV-monitor), a rotatory chair (Pro, Jaeger/Tönnies, Würzburg, Germany), an eye-position detection system either based on DC-electronystagmography or on infra-red reflection (IRIS, Skalar, Delft, The Netherlands), an angular rate sensor (100\% full scale, ARS-C1311A, Watson Industries, Eau Claire, USA). The PC was equipped with an AD-con- 


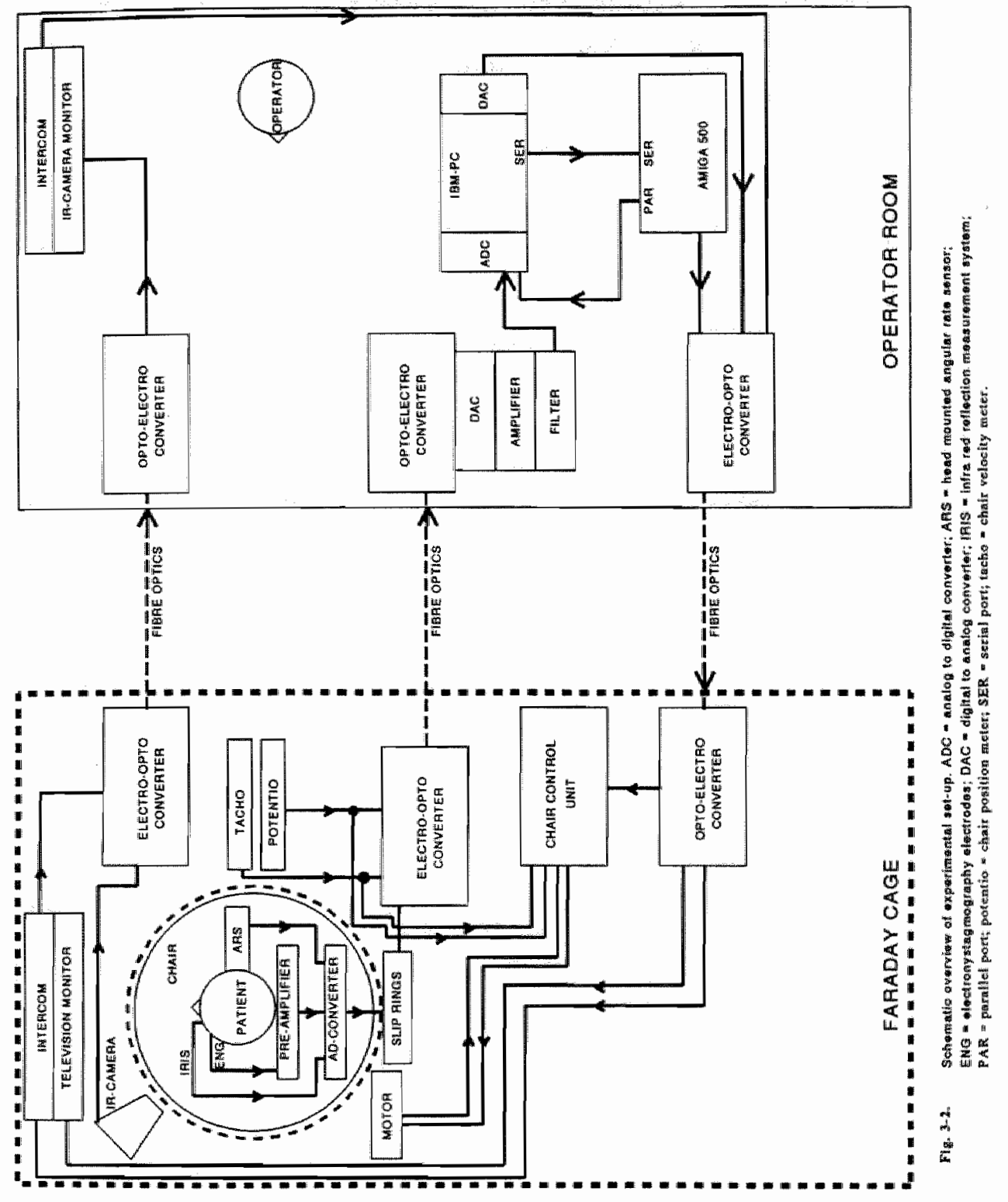


verter ( 8 channel, 12 bits, Burr-Brown PCI-20089W-1, Burr-Brown Corporation, Tucson, Arizona, USA) and a DA-converter (8 channel, 12 bits, Burr-Brown PCI20093W-1). Figure 3-2 shows an overview of the experimental set-up.

Special software (Borland $\mathrm{C} / \mathrm{C}++$ ) was developed for data-acquisition, analysis and stimulus control. The software is commercially available under the name of "Nystagliner" (Jaeger/Tönnies, Würzburg, Germany) and has been used for standard vestibular examination of patients in our clinic since 1990. The IBM-PC acted as the master to control stimulation (chair and TV-monitor), data-acquisition and analysis, while the AMIGA acted as a slave unit of which the local software program (written in Amiga $C$, version 1.3) received instruction through a serial interface from the master and subsequently generated the visual stimulus required. The horizontal and vertical position or velocity of the video-stimulation was generated by the AMIGAsoftware, DA converted from the printer port output and fed into the computer $(A D C)$. The patient was seated on the chair which was placed in a Faraday cage (4 $\mathrm{m} \times 4 \mathrm{~m}$ ). The room was shielded to enable various electro-physiological experiments and because of the unfavorable location of our balance department exactly below the department of radiology. The room could be darkened completely (light-tight). The operator was seated in the adjacent room, where the data-acquisition and stimulus control equipment was located. The patient was illuminated by infra red light and observed by the operator through an infra-red video camera on a monitor in the operator's room; communication was achieved by an intercom system or through instruction displayed on the TV monitor. To preserve optimal shielding all data transfer between the two adjacent rooms was achieved after $\mathrm{AD}$ convertion (16 channel, sample frequency $5000 \mathrm{~Hz}, 12$ bits), conversion into optic signals and transferred through glass fibre optics. Then after passing the shielding the digitized signals were subsequently converted back into electrical pulses and $\mathrm{AD}$ converted into analog signals again.

Because this conversion process was provided as a general feature in the hospital for all Faraday cages, this solution -at least in our case-was inefficient, because the ultimate analog signal had to be converted again using the $A D$ converter in the Nystagliner system.

In case of horizontal and vertical eye position recordings by DC-electronystagmography, general vestibular screening), the biological signals were preamplified $(500 \mathrm{x})$.

All measurements in this thesis were performed immediately after the equipment was installed into the new hospital after removal from the old location. As described in section 3.3 "Calibration" of this chapter, this introduced several complications with respect to the measurements. Partly because of this work all problems have been recognized, located and solved. Several corrections had to be made after a thorough analysis of the data and is described in detail to allow a reliable judgement of the results. 
The rotatory chair was steered by two analog signals generated in the PC (master) and fed towards the rotatory chair control unit via the DAC. One signal was used to set the chair motor electronics to position or velocity control, the second represented either the user defined preprogrammable position or velocity profile as a function of time for generation of the desired chair movement.

Horizontal eye position OD and OS was detected by IRIS (see section 3.3 .3 "Infra red reflection based detection of eye position" of this chapter), amplified (bandwidth $0-30 \mathrm{~Hz}$ ), and fed into the computer through the $A D$ converter.

Chair position was detected by an axis mounted potentiometer, chair velocity was measured by an axis mounted tachometer. Both signals were used for chair movement control. Head movement (which does not necessarily equal chair movement) was detected by a head mounted calibrated angular rate sensor. Also, the position or velocity of the visual target derived from the video output of the AMIGA was fed into the computer through the $\mathrm{AD}$ converter.

All signals were first filtered ( $10 \mathrm{~Hz}$ low pass to prevent aliasing) and fed into the computer through the $\mathrm{ADC}$ using a sample frequency of $50 \mathrm{~Hz}$ (IRIS measurements) or $100 \mathrm{~Hz}$ (electronystagmography).

\subsection{Calibration.}

To make sure that stimuli were given correctly, and that recordings reflected events accurately, the key components were calibrated.

\subsubsection{Angular rate sensor.}

The angular rate sensor is a calibrated device, but because of the unreliable data transmission from sensor to data-acquisition, the complete system had to be recalibrated. This was complicated by the fact that the amplitudes of the chair position and the tachometer signals could not be depended upon, and by that the actual chair movement differed from the PC-instructed one (see below). It was solved by directly recording actual position amplitude of the chair (by fixing a pencil to its foot rest, measuring the length of the written curve and measuring the radius) and by determining the frequency from the angular rate sensor signal. Position amplitude and frequency allowed calculation of the actual maximum velocity, with which the total system could be calibrated. 


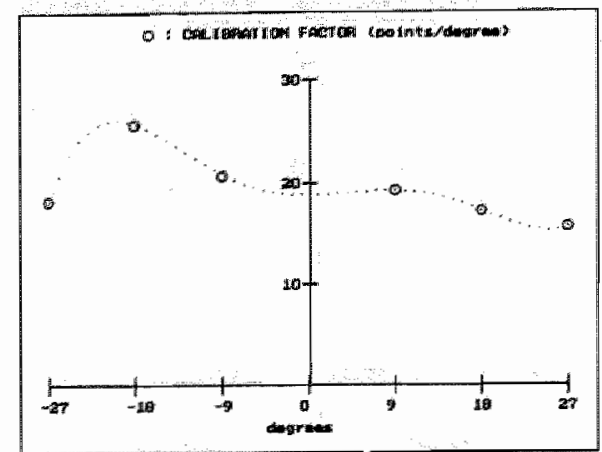

FICAN ENE:

Fugure 3-7. Eye postition dependent callibration factor (neciprocal gain) for the olld IRIS model, immediately after adjustment.

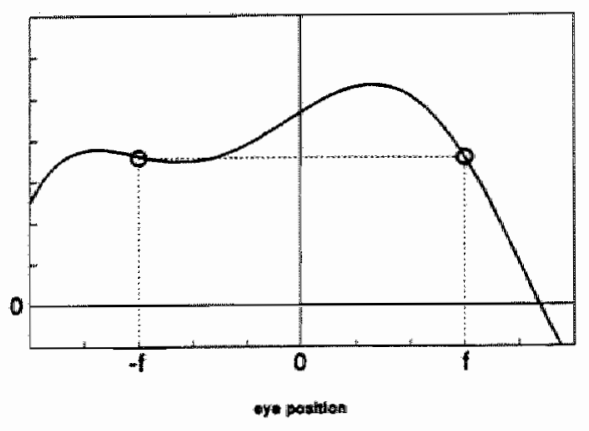

Figure 3-8. During installation/calibration, owtput is made symmetrical when the subject gazes all symmtrically placed fixation points $\left(-f_{*}, D\right)$. This may for the older IRIS model lead to an imoorfect horizontal positioning of the detectors.

$$
\begin{aligned}
& f(x)=\frac{v \times c}{x} \\
& c \begin{array}{l}
\text { cut-off angle } \\
v
\end{array} \\
& x \text { value of calibration factor in flat range } \\
& \text { eyition }
\end{aligned}
$$

Figures 3-5 and 3-6 show how the calibration factor might actually depend on the gaze angle. In figure 3-5 the linearities are shown from measurements with model 6500 at the start, and in figure 3-6 at the end of a session. Initially, the linearity is quite good, but at the end the situation has worsened. In figure 3-7 an initial linearity using the old IRIS model, is shown. This is also an actual setting used for measurements and it is fairly typical for the older model. Three kinds of deviation are seen. First, the two peaks are of unequal height, which can be remedied by shifting the detectors horizontally. Second, the peaks are placed asymmetrically around zero ( $=$ looking straight ahead). This results when the detector is not perpendicular to the line of sight when looking straight ahead. The IRIS is not meant to have this degree of freedom, but due to its construction (tight-coupled joints instead of fixed joints), it does permit such rotation after some weeks of use. The recommended installation procedure gives no clue about this type of misplacement, and, in fact, its exact profile 
can be recognized only after analyzing a registration.

Third, the peaks are not connected by a straight line. So even after making the peaks of equal height, there still is no level range. No way of positioning the detectors could influence this behavior, so presumably it depends on how wide the subject's eyes are opened, in combination with the electronic weighing of the responses of the left and right parts within each detector. The newer version was much improved in this respect.

The combination of the asymmetrically placed peaks and the curved connection between the peaks in the calibration curve, made it impossible to adjust the IRIS correctly by following the recommended installation procedure (figure 3-8). Repeating the procedure for another pair of symmetrically placed fixation points, however, will reveal whether or not the peaks are laterally displaced; if they are, responses will be asymmetrical again. After having gained some experience, reiterating the procedure of calibrating for two different pairs of targets and rotating the detectors a little around their $z$-axes, will ultimately lead to a correct positioning of the detectors.

Calculating alinearity by:

$$
\begin{aligned}
& a=\frac{c_{\max }-\bar{c}}{\bar{c}} \\
& \frac{a}{c}
\end{aligned}
$$

with the old model, we could achieve a linearity of within $25 \%$ in the $[-25 \ldots+25]$ degrees gaze range, and with model 6500 initially within $5 \%$, but over a complete session within $10 \%$. (The range covered by the VVIT is $[-24 \ldots .24]$ degrees: the chair's amplitudes are $10.0^{\circ}, 3.8^{\circ}$, and $1.4^{\circ}$, and the visual target's amplitude is $\left.8.5^{\circ}\right)$.

IRIS responses were not influenced by using an infra red spot light, tungsten spot lights or fluorescent lights (data not shown).

During the measurement period, the total configuration was repeatedly and often without notice, altered by hardware and software specialists. With some delay, a number of such changes were detected because of their effect on the measurement results. In case of altered transmission gain, analysis was first tried with the new calibration factors, and if this still lead to improbable biological gain values for the less recent measurements, "self calibration" was used. In "self calibration", the measurement data themselves are used to derive a calibration factor. Time blocks were defined in such a way, that the subjects' maximum vestibular gains from measurements made within those blocks were most alike, while those measured in adjacent 
blocks were most dissimilar (this was done "on sight", no statistical techniques were employed). For each time block, a calibration factor was chosen, so that the upper quartile of the subjects" maximum responses equaled 1.10 (the theoretical value for a perfect gain when viewing at a distance of $1 \mathrm{~m}$; see appendix 14.2 "Viewing a nearby target ${ }^{\prime \prime}$.

This "self calibration" reduces variation in measured gains, but leaves the calculated gain contrasts (see chapter 5.5), and the measured phase shifts and coherences largely unaffected. In case of altered stimulation, the measurement data were excluded from the study.

\subsection{Frequency analysis.}

Of the digitally stored data, head velocity, visual target position, and left and right eye horizontal eye position were analyzed offline. Target position was differentiated with a 33 data points $(=0.66 \mathrm{~s}$ ) wide window (equivalent to differentiating adjacent points, followed by unweighted averaging over 32 data points) and eye position was differentiated with a three points $(=0.06 \mathrm{~s}$ ) wide window to obtain smoothed velocities. After converting $\mathrm{AD}$-card units to degrees and to degrees per second, stimulus velocity was calculated by subtracting head velocity from visual target velocity. (Subtraction is necessary, as head movements elicit compensatory eye movements, while target

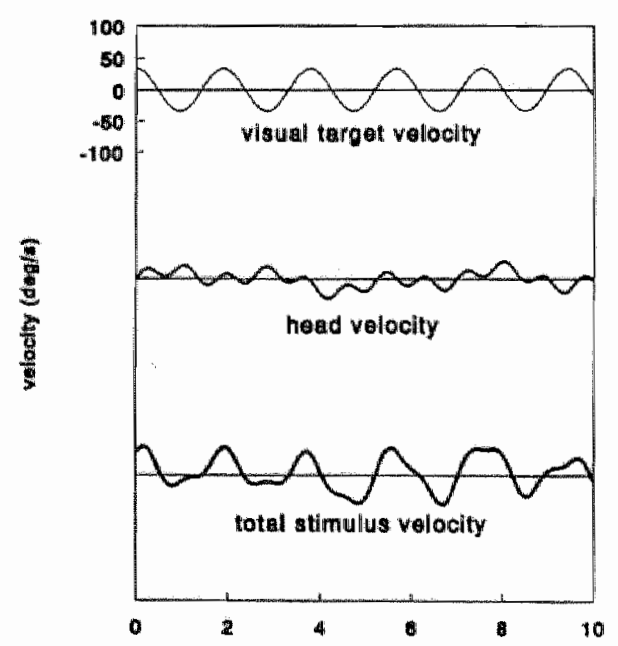

Figure 3-9. Total stimulus (extrat fat line) is composed of singlestine visual target motion (thin line) anta surm-of-sine hesd utotion (intermediste (at linc)

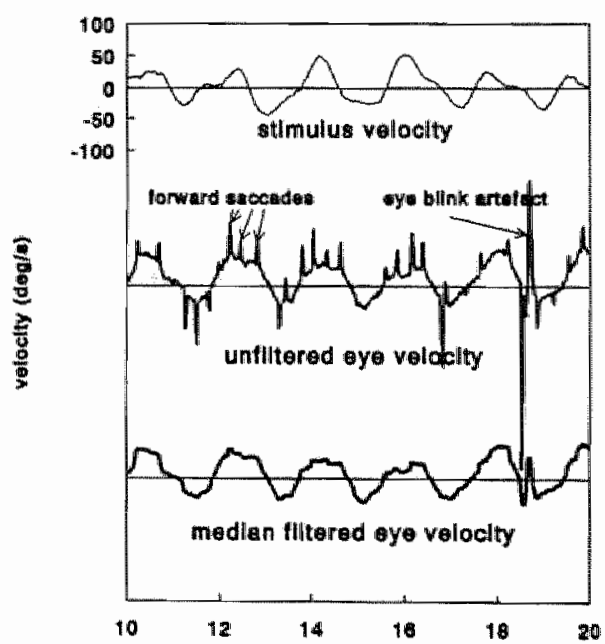

Figure 310. Sum-of-sines stimulus (thin line) and resulting eye velocity (unfiltered: unnermediate tal line; mediats flltered: exra tat line). Forward saccades and an eye blink artefact are indicated. 
movements make the eyes to move in the same direction. One could subtract the target velocity from the head velocity to keep in line with the usual calculations done for rotary experiments in the dark, but in the presence of a visual target the resulting 180 degree phase lead or lag would be rather illogical.) Figure 3-9 shows visual target velocity, head velocity and apparent stimulus velocity.

Although the signals were filtered analogously $\left(f_{c}=10 \mathrm{~Hz}\right)$ before analogue-to-digital (AD) conversion to prevent wrap-around, low frequency parts of the frequency spectrum could still be influenced by fast components and/or eye blink artifacts, necessitating further digital filtering. Earlier attempts to write software for recognizing and removing fast components in the position signal had been unsatisfactory (unpublished results), so it was chosen to smooth the velocity signal. Smoothing by averaging, however, would alter the slow component velocity (and thus the gain) as well, and so median filtering was used instead. The optimal window width was determined experimentally (see appendix "Median filter").

Median filtering the eye velocity signals had two effects. First, the eye blink induced artifacts were reduced, and second, fast components of the eye movements were almost completely deleted. Normally, the fast components were made up of forward saccades only, as the used stimuli did not evoke nystagmus. In case of asymmetrical forward saccades, removing the fast components introduces a shift of the signal. average. The resulting peak at the first frequency bins of the spectrum might obscure the sought response at the lowest stimulus frequency, and therefore any offset of the mean was neutralized before transformation. Figure 3-10 shows apparent target velocity (actual target velocity minus head velocity), unfiltered eye velocity, and median filtered eye velocity, with some forward saccades and an eye blink artefact indicated.

\subsubsection{Data window.}

Fourier analysis assumes signals to be repetitive and of indefinite length. The latter is, of course, impossible in practice. A finite signal can still be analyzed, however, because it is equivalent to the product of an infinite signal with an infinite window function. From the definition of Fourier transformation as an infinite integral of a power function, and of convolution as an infinite integral of a multiplication of two functions, it follows that a multiplication in the time domain is transformed to a convolution in the frequency domain and vice versa. Also from those definitions, it follows that Fourier transformation is distributive and associative. So transforming a finite signal gives the same result as convoluting the transformed window function with the 


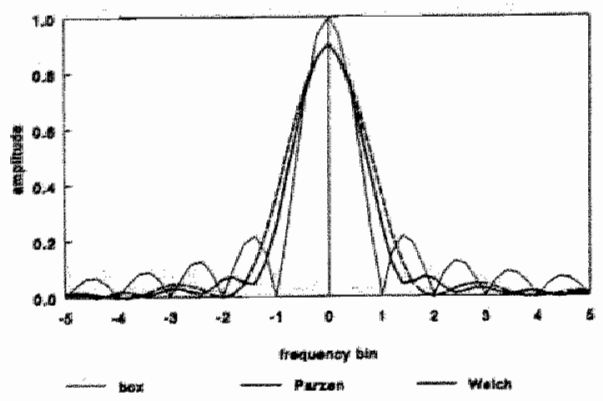

Migune 311. Amplitude spectra of box, Parzen and Welch window functions. The Welch window contributes the least. to neightboring frequency bíns and has a main peak widu in between linose of the box window and the Parzen window. transformation of the endlessly repeated original signal.

The transformation of the box window function is given by the sinc function $(\sin (x) / x)$, which has non-zero elements from minus infinity till plus infinity. This results in poor spectra, as each frequency present in the signal, contributes to almost all the frequency estimates in the spectrum. Therefore, a different window shape is preferable.

Numerous data windows are available, but for most applications (including the cur-
ess important then its width. Welch (1978) rent one), the type of window is much less important then its width. Welch (1978)
recommended to use either a Parzen or a Welch window, which is supported by Press et al. (1988, $\mathrm{p}$ 443). Because the Welch window has less leakage from an offset of three frequency bins on than the Parzen window, as well as a narrower main peak (figure 3-11), the former was chosen.

A Welch window is defined as:

$$
\begin{aligned}
& f_{w}(i)=1-\left(\frac{2 \times i+0.5-w_{w}}{0.5+w_{w}}\right)^{2} \\
& \text { i data point number } \\
& f \text { window factor } \\
& w_{y} \text { window width (in number of data points) }
\end{aligned}
$$

\subsubsection{Settings.}

Frequency analysis of the filtered signals was done by using the fast Fourier transform (FFT) on 2048 data points with a Welch data window to reduce leakage. Having realvalued data only, stimulus and response could be transformed together in one pass.

Median filtering and FFT were different for calculating gain and coherence at the one hand, and for calculating the phase shift at the other.

For calculating gain and coherence, the median filter width was $0.15 \mathrm{~s}$ (nearest higher odd number of data points is 9). Because frequency-specific gain and coherence are calculated using power spectra, it is possible to segment the data, transform each 
segment and to average the results. Further, as data windowing makes less use of the data off center, efficiency can be increased by letting the consecutive segments overlap each other and in case of a Welch window, half overlapping segments are optimal (Welch, 1978). Here, VVIT signals were recorded for 125 seconds at a $50 \mathrm{~Hz}$ sampling rate, so 5 half overlapping segments were available for transformation. Gain was calculated from the averaged power spectra. Coherence was calculated after further frequency averaging was done untill the product of averaged segments and averaged frequency intervals equaled or exceeded 40 (Bendat \& Piersol, 1971, p 191194).

Phase shift is calculated from the real and imaginary parts of the frequency spectrum, and so no spectral averaging is possible. In order to calculate the phase shift over the same time length as gain and coherence were, the digitally stored signal was resampled, so that the total time length fitted in the 2048 points available for FFT. The resulting lower sampling rate brought as an additional advantage a higher frequency resolution (at the cost of a lower frequency maximum, which was unimportant for the VVIT).

The resampling made a different width of the median filter optimal, for at small numbers of data points, median filter effect depends on number of data points as well as on time length. The width was $0.35 \mathrm{~s}$ (nearest higher odd number of resampled data points is 7 for a recording time of 125 seconds).

The gain, phase and coherence at the response frequencies were extracted automatically from the spectra, using preset criteria.

First of all, the program decided whether the spectrum had sufficient relief: the highest value should be more than 100 times the lowest value. This was necessary to prevent analyzing a level (but noisy) spectrum, as the peak threshold (see below) was not an absolute value.

Next, any local (ie., current position \pm 1 bin) maximum was required $a$. to be above

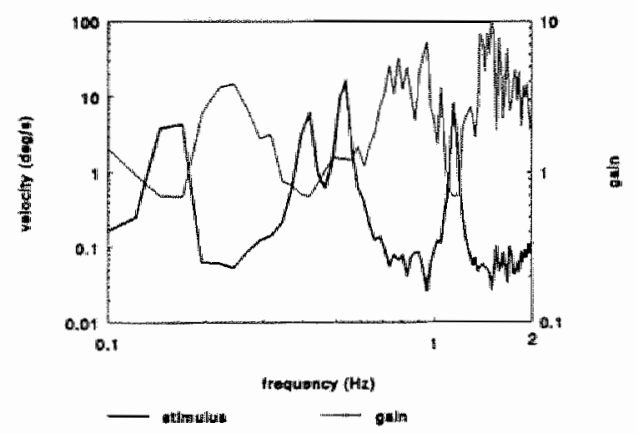

Figure 3-12 Spectrum oblained by power spectrum averag ing. showing suimulus welocity (fat line) and subject's gain (thin line). Chair three equal stimulus preaks at $0.16,0.42$ and $1.15 \mathrm{~Hz}$; visual target: higher one all $0.53 \mathrm{~Hz}$

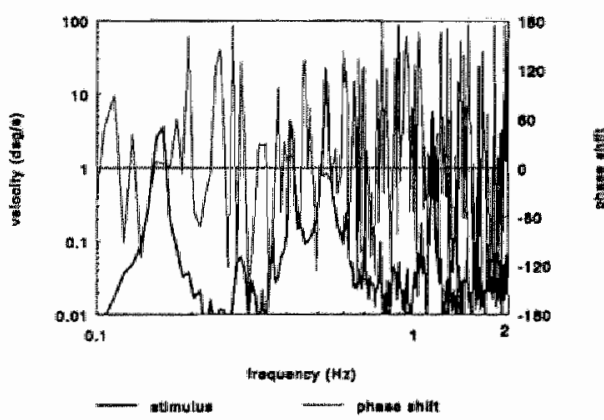

Fügure 3-13. Spectrum from resamplled, recorded data, showing stimulus (fal line) and please shift (thin line). Same signal as in figures $3-12$ and $3-114$. 


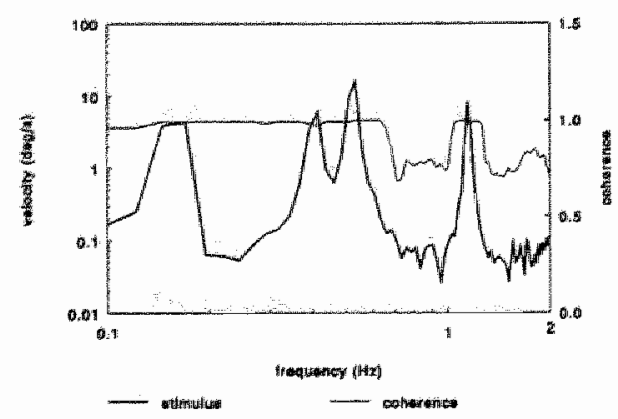

Fiture 3-14. Spectrun obtanded by power spectrim averag. ing, showhing shimatus (fal line) and frequency-averaged subject's coherence (thin line). Same signal as in Ggures 3 . 12 and $3=13$.

the threshold of $10 \%$ of the spectrum's maximum, and either $\underline{B l}$. to be at least twice as high as the local (ie., current position \pm 5 bins) minima at both sides, or b2. to be at least twice as high as one side's local minimum and to be of about the same height (next highest $>0.8 \times$ highest) as the other side's local maximum. Condition $a$ selects against noise peaks, and condition b1 against noise shoulders, while condition b2 retains twin peaks nevertheless.

The remaining peaks were selected further using their corresponding gain and coherence values. Gain had to lie within the $[0.05$ . 1.5] range, and coherence within $[0.1$.. 1.5$]$. The gain criterium removed most noise-based peaks from the selection, especially those at the higher frequencies, while the coherence criterium had little effect.

Finally, it was checked whether the so found peaks were at or near the frequencies delivered during stimulation. Normally, those were the only ones found, but occasionally other frequencies appeared. (In case other frequencies appeared instead of the expected ones, subsequent inspection of the original data showed that in those cases chair and/or visual target movement had been different indeed, and they were excluded from analysis. And in the last quarter of the study an extra peak appeared in the lowest frequency bin. This could be traced to a chance in hardware: the amplifier for the angular rate sensor had been replaced by one with a non-correctable offset. This offset was digitally removed as described above.) Figures 3-12, 3-13 and 3-14 show gain, phase shift and coherence spectra, respectively, with the stimulus spectrum. It can be seen that at the frequencies where is stimulated, the calculated parameters gain and phase shift attain values within the expected range, while in between those frequencies they do not, due to noise correlations. The coherence, however, is never lower than 0.6 , even at frequencies where noise dominates, suggesting that perhaps $\gamma^{2}$ should be used instead of $\gamma$, as is done here, or that frequency-averaging should not be done over more bins than the width of the range of interest. This has not been investigated further.

\subsubsection{Validation.}

The software for Fourier analysis was adapted from Press et al. (1988), and was 


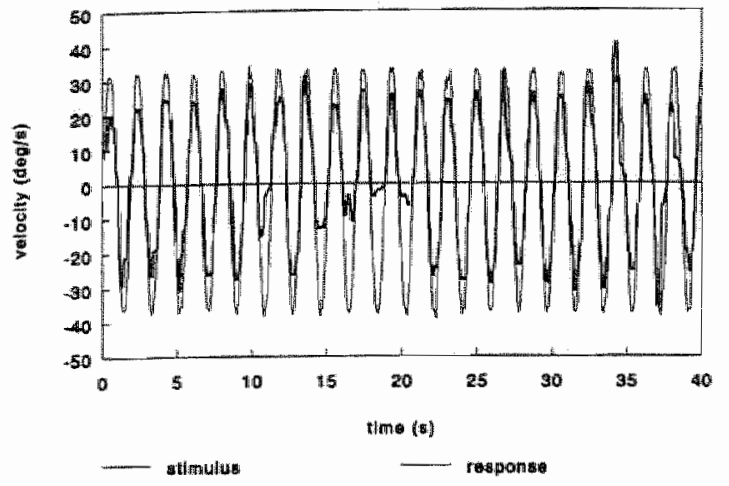

Fyque 3-15. Stimulus (thin line) and nesponse (thich line) vetocities of a sinussoidally ( $=0.53 \mathrm{HL}, \mathrm{A}=10$ degres) moving target, viewed by a normal, but tires, subject. Phase shift is negligible and gain varies in time petween 0.55 and 0.80 .

supplemented with the calculation of the coherence as given by Bendat \& Piersol (1971, p 337-339). It was tested on artificial data and was found to estimate the gain and phase shift accurately (data not shown). Without having checked the coherence values, we think their relation (but not the absolute values themselves; see above) is reliable too, for two reasons. First, the coherence is derived from the same spectra as are used to calculate gain and phase, which were found to be correct. And second, for rotary chair experiments, the obtained values fall within the expected range of $[0.8$ .. 1.0』 (for the head shake experiments, much lower values were also obtained, which is not surprising, considering the sometimes very severe non-stationarity and nonperiodicity).

The complete analysis (median filtering, FFT, gain and phase calculations) was also tested on actual data. Because it would be rather difficuit to check the overall analysis of pseudo random actual data, a simple sinusoidal visual stimulus of $0.53 \mathrm{~Hz}$ and 10 degrees was used. Routine calibration was done by the measuring program (averaging five saccades for $10^{\circ}$ position steps) and the routine settings for the analyzing software were used.

The obtained phase shift of -2 degrees (corresponding time shift of 0.01 second is less than the sample period of 0.02 seconds) agrees well with the visual impression of no phase shift (figure 3-15). The obtained gain of 0.74 agrees reasonably well with the gain (0.69) derived by minimizing the root mean square error (RMSE) and very well with the gain ( 0.73$)$ derived by minimizing the mean absolute error (MAE; error was defined as the observed eye velocity minus the product of the gain with stimulus velocity) (figure 3-16). Because the signal was not stationary (figure 3-15) and the RMSE weighs large errors heavily, it is not surprising that minimizing the RMSE led to a gain that is somewhat lower than the one produced by frequency analysis. This is illustrated in figure 3-17 which shows the scatter plot of eye velocity versus target velocity, with the two regression lines. Clearly the regression by means of the least 


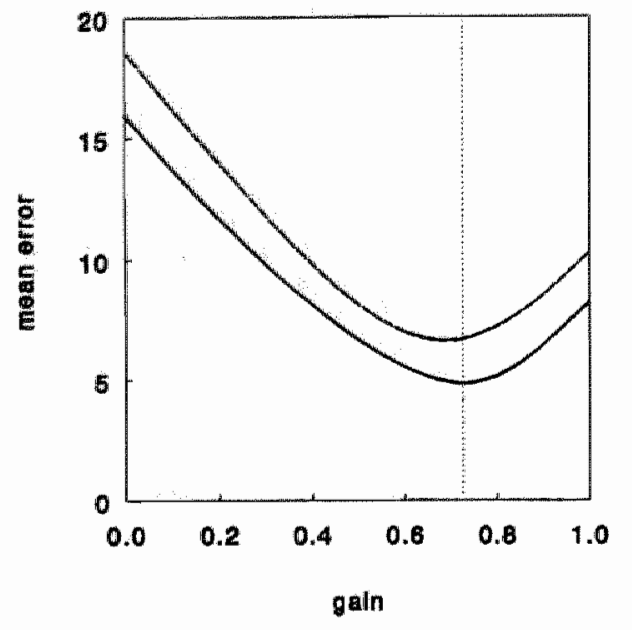

Wugure 3-16. Minimizing the root mean square error (RMSE) and the mean absolute fror (MAE) produces gains of 0.69 and 0.73 , respectively, which are somewhal lower than the walue 0.74 . (indicated by the cloted line) fownd by frequency analysis.

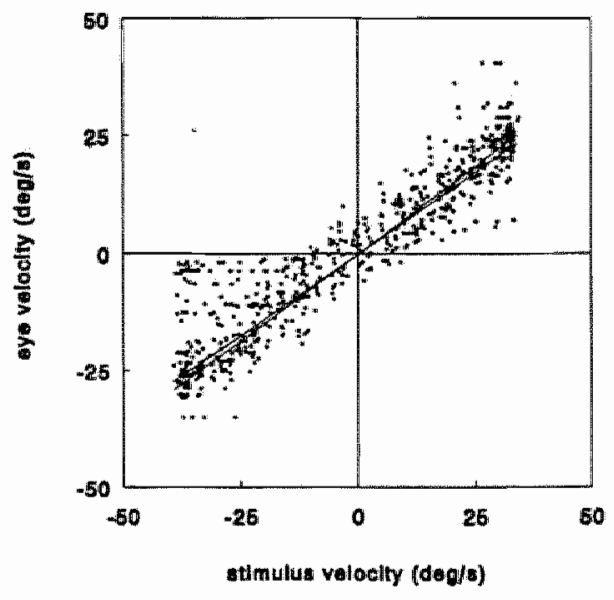

Figure 3-17. Scatter plon of eye velocily versus visual target wellocity of the same sipnal as used for figures $3-15$ and $3-16$. with regression lines based on the lleast squares methout (sllope 0.69 ) and on the absolute errors (slope $=0.73$ ).

squares method is strongly influenced by the deviant values in the upper part of the lower left quadrant, while the regression line based on the absolute values of the errors follows the main course (area with highest density of values) more faithfully. It is concluded that the calculation of the gain in the Fourier domain after median filtering in the time domain of data obtained by single frequency stimulation produces reliable estimates, even if not all requirements for Fourier transformation (stationarity and periodicity) are met. It is assumed without further checking that the same will hold for data obtained by multiple frequency stimulation. 


\section{Slow component characterization.}

Standard vestibular tests are used to compare the new Visuo-Vestibular Interaction Test (VVIT) with. And because standard tests provoke nystagmoid eye movements, this made an algorithm desirable for the automated characterization of slow components.

Two strategies are available. Per slow component a single averaged velocity can be computed, resulting in irregularly spaced data through which a function might be fitted. Alternatively, fast components can be replaced by an estimate of the slow component.

\subsection{One averaged velocity per slow component.}

First the separate components have to be distinguished. We use a modified version of the min-max method (Owen Black \& Wall III, 1984, partly based on Honrubia et al., 1971). Originally, in this method a window steps over the data in such a way, that in each position its left border conjoin with the right border of its previous position. In each position, the current lowest value is remembered for the next position, if the previous extreme was a maximum, or conversely, the highest value is remembered if the previous extreme was a minimum. As long as the lowest values keep getting lower, or the highest values higher, the window steps on. When a change in trend appears, the remembered value is stored as an extreme. It is labeled to be a maximum for a change from higher to lower, or a minimum for lower to higher. We have made five changes. First, the window is repositioned with its left border on a new found extreme. This speeds up processing, as no superfluous searching for the opposite extreme is done in front of the one last found. Second, the velocity of a component (i.e., the slope of the part between two extremes) is approximated by linear regression (instead of using the slope of the line connecting the two extremes), as used by Juhola et al. (1985) in their adaptive filter. This gives a less biased estimate in case of noise, and it allows a fidelity check (see below). Third, labeling of a component as fast, is based on comparison of the multiplicative products of velocity and position amplitude for two adjacent components, instead of comparing only velocity. Although the original way is attractive for its conceptual simplicity, using the product gives better results for signals with a varying degree of noisiness, because it makes the amplitude threshold less critical. Fourth, if two adjacent components both are marked to be fast, then both are removed. In this way, no separate spike detection algorithm is needed, and noise influences at the extreme eye positions (the 
maxima and minima of the sinusoid cumulative eye response) are diminished. Fifth, the distributions of the velocities and the correlation coefficients of the three previous and the three following potential slow components is used to judge the current one. This one is rejected if it digresses more than two standard deviations from the means. Obviously, this method can work only when the eye movements are nystagmoid, and it provides sparse data, but those few data points are rather reliable.

\subsection{Replacement of fast components by using nonlinear filters.}

Our earlier attempts to construct a cumulative eye position signal by removing fast components failed, because they could not be identified 100\% correctly (unpublished results). Therefore, we now concentrated on filtering the velocity signal. For the VVIT signals, infrequent spikes caused by eye blinks and saccades had to be removed, suggesting non-linear filtering would be appropriate.

An important class of non-linear filters is formed by rank filters. They do not perform some linear operation on the data contained within the window (as do linear filters like the running-average filter), but they use rank information to produce output. First, the generally used median filter is described, and next our generalization of it: the variable percentile filter.

In statistics, three measures are well known to describe the center of a set of data: the mean, the median, and the modus. And so it is only natural after having seen a running-average and a median filter, to try a filter that produces the modal value as its output.

The asymmetry study of chapter 12 "Discriminating power of the Visuo-Vestibular Interaction Test" suggested another way of median filtering, namely of stimulusordered data. Because of the hysteresis present in the response to a steady-state sinusoidal stimulus, a thybrid ranking-averaging filter (a rank-trimmed moving-average filter) was also tried. 


\subsubsection{Median filter.}

Median filtering is a nonlinear technique much used in digital image processing. Depending on its window width, such a filter rejects spikes, follows ramps faithfully and sharpens steps (among others, Hodgson et al, 1985).

Median filtering is done by moving a window over the data. At each window position, the data values are ordered in magnitude, and the middle value is output as the new data value. In case the filter width is an even number of data points, the output is formed by the average of the two middle values (but this introduces a small phase shift which might become important when the filter is applied repeatedly). In order to prevent recursion, a buffer the size of the filter is used to hold a shifting subset of the original data points.

Depending on the situation, three scales are appropriate for evaluating the width of a median filter.

For very small widths, the behavior of the filter is heavily influenced by the number of data points, making this a good scale. For intermediate widths, it should be several times the (fixed) duration of the fast components that have to be rejected, irrespective of the sample frequency. And so time would be better than number of data points in describing filter width. For very large widths, finally, the ratio of the filter width and the (variable) period of the stimulus determines the effect, as clipping of the wanted signal might occur. From this it follows that the width of the filter is a compromise between conflicting demands. It should be large, so that extreme values are excluded well, and at the same time it should be small, so that even the highest stimulus frequency is not attenuated. Systematically varying the width for filtering actual measurement data, showed that a window size of about one-tenth to one-fifth of the shortest stimulus period is optimal (figure 4-1) for our data. With smaller ones the gain can be disturbed by eye blinks, while with larger ones the gain drops below its actual value because the slow component itself gets clipped. This latter effect, however, probably can be cancelled by filtering (and thus clipping) the stimulus signal in the same way as the response signal, but this might affect the phase shift and/or the coherence in an unwanted way.

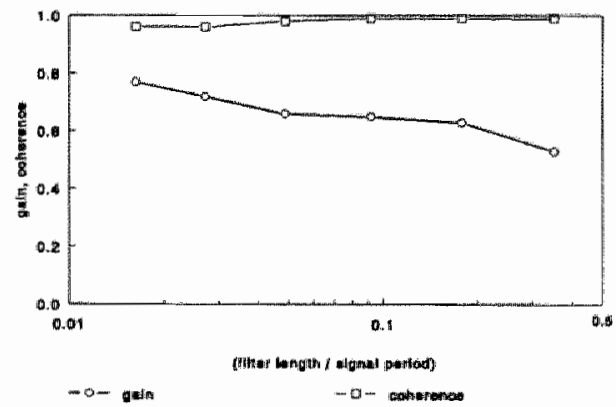

Figure 4-1. Effect of median filuer width on calculated gain and coherence for actual measurement dala. Beich a ratio of 0.04 , disturbances still influence gain, while above 0.15 , clipping of the signal occurs. 


\subsubsection{Variable percentile filter.}

For visuo-westibular interaction, the simple median filter suffices for removing saccades and attenuating eye blink artifacts, as both are relatively rare compared with the desired part of the eye velocity signal. But when eye movements in the dark (e.g., calorization, velocity step or torsion in the dark) are analyzed, fast components (saccades, nystagmus beats) may constitute a considerable part of the total signal, and so they can influence the filter output strongly. Furthermore, the disturbing fast comFugume 42 Companison of median filtered data (lower panel, continous line) with mystagmus-recognizing via min-max method
(lower panel, discnete points).

Upper panel (OD HOR) = horizontal right eye position; second panel (OD VER) = vertical right eye position; third panel (STOEL SNELFI) = chair velocity lower panel $(\mathrm{OD} H \mathrm{HOR})=$ calculated horizontal right eye velocity. 
ponents are not random, but are opposite to the wanted slow component eye velocity signal.

In these cases, two requirements of the median filter are violated: $\underline{a}$ a large part of the signal is corrupted and $\underline{b}$. within the filter window the deviations are highly asymmetric. If the asymmetry is consistent, then it can be countered by making the filter opposite asymmetric, Le., by not outputting the median value, but an other percentile value like that of the first or third quartile (the median is here considered to be the second quartile). This works fine for the initial stages of the velocity step and for the cumulation period of the calorization, but when the nystagmus frequency slows down, the filter asymmetry should diminish too. In case of torsion in the dark, the situation is even more complicated, as the asymmetry reverses repeatedly.

Therefore, we generalized the median filter into a variable percentile filter. Choosing which percentile should give output, can be based on the data enclosed in the current filter window (giving an internal or self varying filter) or on external criteria (e.g., depending on the stimulus). The number of percentiles can be one (a fixed percentile filter, e.g., outputting the $50 \%$ value (median filter)), two (a bi-stable percentile filter, e.g., outputting the first or the third quartile) and so on, up to semi-continuous (not really continuous, as the filter width is an integer of discrete data samples). In order to retain the attractive property of being robust against wild outliers, it is advisable to limit the lower and the upper percentiles that may produce output.

When making a percentile filter self-varying, one has to specify a link function which links a percentile to the data characteristics. An obvious choice is to compare the values at two symmetrically placed percentiles with the median value to asses the amount of data asymmetry, just as is being done in a box-and-whisker plot (Tukey, 1977). We use the contrast (i.e., the relative difference) to make the link function:

$$
\begin{array}{r}
p=m+\frac{v_{d, 1}-v_{d, 2}}{v_{d, 1}+v_{d, 2}} \times w \\
v_{d, 1}=v_{m}-v_{q, 1} \\
v_{d, 2}=v_{q, 3}-v_{m}
\end{array}
$$




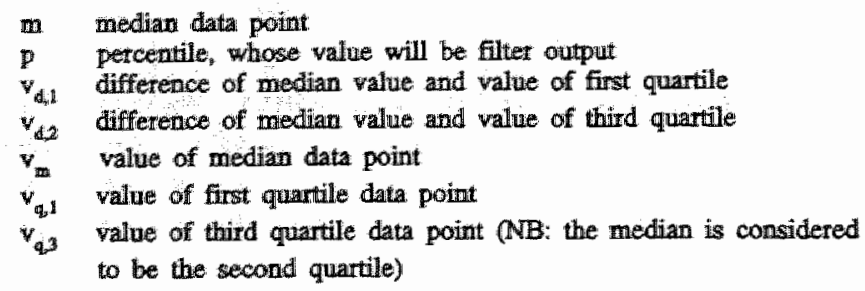

With the first and the third quartile as limits, this filter worked rather well on differentiated, $100 \mathrm{~Hz}$ sampled eye position signals. Occasionally, however, the filter incorrectly flipped shortly over from lower-than-median percentiles to higher-thanmedian percentiles or vice versa, producing a sharp spike. These occasional spikes can be removed afterwards by an additional pass of a simple median filter. Because percentile filters tend to produce staircase-like output when rejecting spikes on a slope, finally the signal is smoothed by a moving average filter.

In figure 4-2 the thus filtered signal is compared with slow component velocities obtained by recognizing a nystagmus pattern in the position signal and approximating the slow components by straight lines. As long as slow and fast components differ at any time, both methods produce similar values. The percentile filters, however, provide a continuous signal that includes the zero crossings, while the nystagmus recognizing algorithrn only can give values at discrete points at the nystagmus frequency. At the other hand, the nystagmus recognizing algorithm can still correctly characterize an occasional ambiguous slow and fast component pair, because it uses semantics from a larger region, or it can discard a pair as unreliable.

\subsubsection{Modal filter}

The self varying percentile filter adjusts itself to asymmetric data distributions by assessing the asymmetry and by outputting from a counter-asymmetric percentile. A similar effect should be obtainable in a more direct way by outputting the modal value. To do this, a histogram (with rather course classes; say, five to cover the whole data range) is made of the data enclosed in the filter window, and the mean or the median of the best filled elass is output.

Modal filters and variable percentile filters will work totally different, however, on signals with more than one modus per window. With an almost bimodal distribution in its window, a modal filter will still output from the best filled class, while the variable percentile filter will output the overall median value. This is exactly the situation referred to at the end of the previous paragraph: the absolute values of the 
slow and of the fast component are rather similar. Trials showed that the modal filter did indeed perform better than the variable percentile filter at very high nystagmus frequencies, but at the cost of a long processing time. This drawback will become less with more powerful computers.

\subsubsection{Stimulus-ordered median filter.}

In case of a linear system responding to a single-sine stimulus, the plot of response versus stimulus shows an oblique ellipse, of which the tangent of the angle of the main axis gives the gain and of which the width at zero stimulus is twice the product of gain, stimulus amplitude, and the sine of the phase shift:

$$
\begin{gathered}
{\left[\begin{array}{l}
x=A_{x} \cdot \sin (\omega \cdot t) \\
y=A_{y} \cdot \sin (\omega \cdot t+\varphi)
\end{array}\right.} \\
y=g \cdot x \cdot \cos \varphi \pm \sqrt{g^{2} \cdot A_{x}^{2}-g^{2} \cdot x^{2}} \cdot \sin \varphi \\
-A_{x} \leq x \leq A_{x} \\
g=\frac{A_{y}}{A_{x}} \\
y_{x=0}= \pm g \cdot A_{x} \cdot \sin \varphi
\end{gathered}
$$

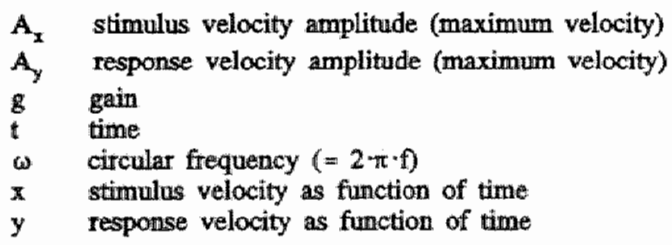

Figure 4-3 shows such a stimulus/response velocity ellipse, with position and acceleration information added to aid interpretation. An actual scatter plot of median filtered sum-of-sines data is given in figure 4-4. It is the widest plot obtained during the study, presumably because the initial filtering did not remove all fast components. This poses hardly any problems for subsequent frequency analysis, but it does seriously hamper analysis of left/right asymmetries because linear regression by the least squares method is very sensitive to outliers. Therefore, the stimulus-response pairs were sorted according to the stimulus velocities, and within these, according to the response velocities. These stimulus-ordered response velocities were median 


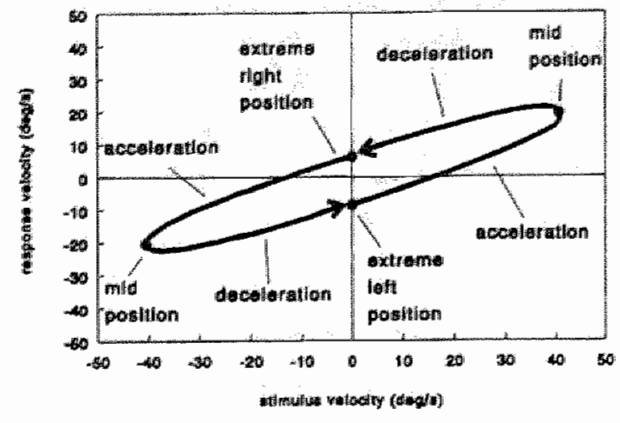

Figure 4-3. Phase shifted response vellocity $y=A, \sin (\omega)$ $+\varphi$ ) versus stimulus velocity $x=A_{x}$ sin tain. Position and acceleration information is included to facilitate interpreta: tion. Main axis: engle indicates gain, width indicates phase shift. filtered (with a width of 25 samples; perhaps an other width will be optimal). Linear regression on these filtered data allows accurate assessment of the asymmetry in left- and rightward velocities, and such results are reported in chapter "Discriminating powers of the VVIT compared to those of the standard tests".

Figure 4-5 gives a line diagram of the thus median filtered data after they had been brought back into their original (chronological) order. Here, the high-low alternations draw attention immediately. They result from the fact that other velocities have been sampled during acceleration than during deceleration, in combination with the median filter's sharpening properties. So they allow assessment of the (frequencyaveraged) phase shift by measuring their peak-valley distance. In this particular case, a distance of $18 \%$ is found, corresponding to a phase shift of about 19 degrees, which is in good agreement with the values obtained by Fourier analysis.

Currently, we have used this method on ordinary median filtered data only. But we expect it to work equally well on raw data, because all outliers removed by the first median filter surely should also be removable by the second median filter.

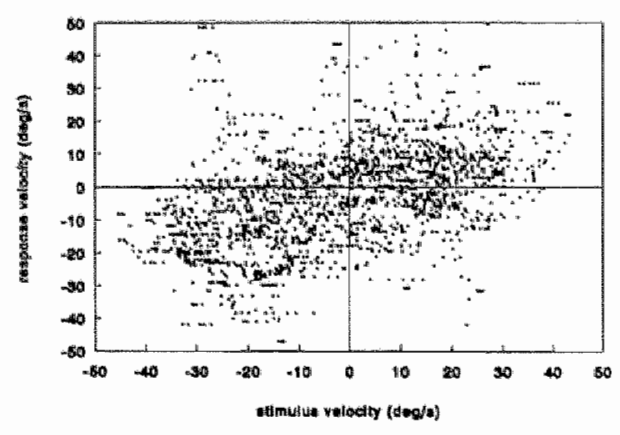

Figure 4-4. Scatter diagratin of a patient's median filtered response to $\mathrm{m}$ (VVTT. The wide variation in response velocities per stimullus welocity probxably resultis from incomplete removal of last components.

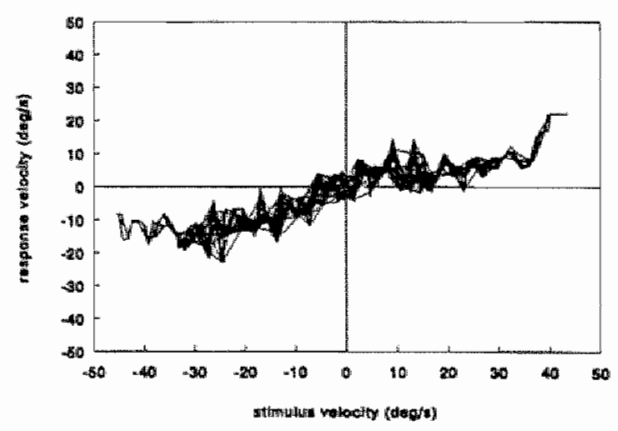

Figure 4-5. Line diagram of chronologically reordered results of median filtering stimulus-ordered response velocities (stme data as in figure 4-4). High-low alternations indicate (frequencyuarergiged) phase shift of 19 degrees. 


\subsubsection{Rank-trimmed moving-average filter for stimulus-ordered data.}

Figure 4-5 also suggests a new way of defining stimulus-dependent thresholds for filtering fast components out of eye velocity recordings. By filtering the ordered data with rather wide maximum- and minimum filters the envelope can be obtained, which can be used to derive the thresholds by adding an offset or by multiplying with a factor. Thus in rejecting the data outside the thresholds, the stimulus velocity too is used and this is in contrast to most rejection schemes, which use the response signal only. A faster way would be, to use a rank filter to define a sub set of data (say, between the first and the third quartile), and to output the average from this. 


\section{Effect of asynchronous visual stimulation on the Vestibulo-Ocular Reflex gain of normals.}

\subsection{Abstract.}

A vestibular function test (named Visuo-Vestibular Interaction Test: VVTT) is presented, in which a visual target may move asynchronously with a pseudo-randomly oscillating rotatory chair.

Here, normative data are given for the relative difference of gains obtained by stimulation with asynchronously moving visual target and with an earth-fixed visual target, while phase shift and coherence are considered elsewhere (chapters 6 and 7 , respectively). Intra-individual variation was found to influence responses much stronger than stimulus frequency or inter-individual differences, allowing one mean value to be calculated.

\subsection{Introduction.}

In order to study vestibular functioning, usually eye movements are measured while the subject's head is moved. One of the processes at work there, is the VestibuloOcular Reflex (VOR), which has evolved to stabilize retinal images by countering head movements through compensating eye movements. Other processes are the Smooth Pursuit (SP) and the OptoKinetic Reflex (OKR), both visually guided. Often to preclude the latter two, testing is done in the dark in an attempt to isolate the "pure" VOR. Two reasons have emerged, however, to study Visuo-Vestibular Interaction (VVI) and to awoid testing the VOR in the dark. First, the VOR in the dark is very strongly influenced by the subject's mental setting (Barr et al., 1976; Bahil er al., 1980; Bloomberg et al, 1991b; among many others). The gain can be changed at will from 0.35 to 0.95 , by imagining a visual target is moving with the head, or imagining it is earth-fixed, respectively. And second, VOR recalibration takes place within minutes (Collewijn et al., 1983), which keeps the system parameters well-defined when a visual target is present, but which might have unknown effects when testing in the dark.

Usually, VVI is studied by either using an earth-fixed visual target (eftVOR, or visually enhanced VOR), or using a chair-fixed visual target (cftVOR, visual suppression, or visual cancellation) (e.g., Barnes et al., 1978). Because the latency of VOR cancelation is shorter than that of the visual system (Cullen et al., 1991), however, it has been suggested that there might no interaction under those circumstances, but that a VOR self-cancellation might be switched on and off. Therefore, we use a visual target that moves asynchronously with the chair, thus hoping to force an interaction 
between the visual and the vestibular systems.

By choosing the frequencies and amplitudes in such a way, that the peak velocity of the chair is around the upper limit of the SP system (circa $30 \% / \mathrm{s}$; Honrubia $e t a L_{\text {, }}$ 1968), even the least responsive vestibular patient should be able to compensate chair movements almost perfectly by visually tracking the apparent target movements in case of an earth-fixed target. During the visuo-vestibular interaction with an asynchronously moving target (mtVVI), however, it is expected that only a normal subject can move his eyes well. For his vestibular system will compensate the chair movements sufficiently, so that the SP system has to deal with the actual target movement only: Without vestibular gaze stabilization, the apparent target velocity exceeds the SP system's upper limit, and therefore we expect the vestibular patients to perform. worse. So we subtract the responses to only chair movements from the responses to combined target and chair movements, which leaves us with the visual contribution. We hypothesize that this can be interpreted as indicating the vestibular functioning: The information about vestibular functioning is then assumed to be contained by the ratio of smooth and saccadic eye movements (which sum up to a gain of unity). By filtering the fast components out of the response sigmal, the smooth part remains, and it is for this part that the above-mentioned arguments might hold.

It is assumed here, that vestibular stimulation will result in only smooth eye movements, not in saccadic ones. Bloomberg et al. (1991a, 1991b), however, reported that subjects who tried to look in the dark towards a remembered position while being rotated, used both smooth and saccadic compensatory eye movements in a synergistic way. As long as their ratio remains constant, this would only diminish the VVIT's sensitivity, but it would not bias the results.

\subsection{Material and methods.}

\subsubsection{Subjects.}

Six controls ( 3 males of 23,33 and 37 years, and 3 females of 23,26 and 34 years) were recruited among ourselves and our students, resulting in a lower median age than that of the usual patient population for our clinic.

None of the subjects had any history of vestibular problems, and none of them had any difficulty with visually guided eye movements. All subjects, even those usely wearing glasses, could locate the visual target without any optical correction, and were therefore tested that way.

Three of then (two males of 23 and 37 years, and one female of 26 years) underwent the VVIT each at four different days in order to asses intra- and inter-subject variability. 


\subsubsection{Experimental set-up.}

Subjects were seated in a rotatory chair (P-chair, Tönnies/Jaeger, Würzburg, Germany), watching a dot (29 minutes of an arc in diameter) on a television screen (28CE6290, Philips, Eindhoven, The Netherlands) $100 \mathrm{~cm}$ in front of them. The chair was controlled by an IBM-compatable personal computer (PC), and moved in a pseudo-random way. Its velocity was the sum of three sinusoids of frequencies of 0.16 $\mathrm{Hz}, 0.41 \mathrm{~Hz}$, and $1.15 \mathrm{~Hz}$, with each having a maximum velocity of 10 degrees per second. The visual stimulus was generated by a micro computer (Amiga 500, Commodore, Germany), which was also under control of the PC. The dot moved in a sinusoidal way along a horizontal path with a frequency of $0.53 \mathrm{~Hz}$ and a maximum velocity of 32 degrees per second. Note that the frequencies are not only harmonically unrelated (Stark et al., 1962), but that also their pairwise sums (except one) and differences are no multiples of the fundamental frequencies (Maki, 1986, 1988). Apart from the television set, of which the background was set as low as possible, the room was completely dark.

Two measurements were made. First, the chair moved in a sum-of-sine fashion, while the visual stimulus was stationary with respect to the earth. This was called the earthfixed target Visuo-Vestibular Interaction (eftVVI). And second, the chair moved in exactly the same way as the first time, but now the dot moved asynchronously with the chair. This was called the moving target Visuo-Vestibular Interaction (mtVVI).

Head movements were measured by an angular rate sensor $(100 \%$ full scale, ARSC131-1A, Watson Industries, Eau Claire, USA), worn by the subjects using a tightly fitting head assembly. At the same assembly the IRIS system (Scalar, Delft, The Netherlands) was fixed, measuring eye positions by means of reflection of infra-red light (alinearity of 10\%).

Left and right horizontal eye position, head velocity, and visual stimulus position were sampled at a $50 \mathrm{~Hz}$ frequency for 125 seconds by a 12 bit analogue-to-digital (AD) converter (the first three after being filtered analogously with a cut-off frequency of $10 \mathrm{~Hz}$ ) and were digitally stored for offline analysis.

\subsubsection{Digital signal analysis.}

Target position and eye position signals were differentiated with a window 33 data points and 3 data points wide, respectively, to obtain smoothed velocities without introducing a phase shift. After converting $\mathrm{AD}$-card units to degrees and to degrees per second, stimulus velocity was calculated by subtracting head velocity from visual target velocity. 
Fast components and eye blink artefacts were renowed or attenuated by using a median filter with a width of 0.15 seconds (i.e., 9 data points). The median filtered signals were transformed using a 2048 data points wide Fast Fourier Transform (FFT) algorithm (Press et al., 1988, p 407-412, 445-446) after windowing with a Welch filter (Press et al, 1988, p 441-444). For each recording a gain spectrum was calculated from averaged power spectra, obtained by combining the power spectra from 5 half overlapping time segments (Press et al., 1988, p 445). The gain values at the response frequencies were extracted automatically from the spectrum using preset criteria. It was checked that these frequencies were the same ones as was stimulated at.

The individual contrasts (see appendix) of the eftVVI and the mtVVI measurements were calculated and these were also analyzed statistically.

\subsubsection{Statistical analysis.}

Responses were analyzed for stimulus frequency, subject, and the interaction between frequency and subject, by multivariate analysis of variance (MANOVA; repeated measurements design; Wijvekate, 1972, p 181-184). The limits of the reference intervals (Albert \& Harris, 1987) were calculated from the means and the standard deviations by:

$$
\begin{aligned}
& \bar{x}-t_{0.975, d f} \cdot s^{\cdot} \sqrt{1+\frac{1}{n}}<x_{n+1}<\bar{x}+t_{0.975, d f f^{*}} \cdot \sqrt{1+\frac{1}{n}} \\
& \begin{array}{l}
\text { group size } \\
\mathrm{s} \quad \text { estimated standard variance }
\end{array} \\
& \text { 60.973, af } 97.5 \text { percentile of the Student distribution with } \\
& \text { df cegrees of freedom } \\
& \bar{x} \quad \text { estimated mean (using } n \text { values) } \\
& x_{n+1} \text { independent, next value }
\end{aligned}
$$

Royston \& Matthews (1991), however, have argued that

$$
\begin{aligned}
& \bar{x}-z_{\alpha} s / \lambda<x_{n+1}<\bar{x}+z_{\mathrm{e}} s / \lambda \\
& \lambda \text { unbiasing factor (depending on group size) } \\
& \text { n group size } \\
& \text { s estimated standard variance } \\
& x_{\alpha} \text { the } 100^{\text {n }} \alpha^{\text {th }} \text { centile of the standard Normal distribution } \\
& \vec{x} \text { estimated mean (using } n \text { values) } \\
& x_{n+1} \text { independent, next value }
\end{aligned}
$$


gives unbiased estimates of the end points $\mathrm{C}_{\alpha}$ end $\mathrm{C}_{1-a x}$ and that

$$
\bar{x}-z_{\alpha} \cdot s<x_{n+1}<\bar{x}+z_{a} \cdot s
$$

results in a bias and a mean square error in between those of the formula giving unbiased estimates and one producing minimal mean square error. According to these formula's, the here used limits do not give a 5\% error probability, but one of only $0.83 \%$ and $0.55 \%$, respectively. Those authors explicitly disapprove, however, of calculating any reference range based on small sample sizes (e.g., less than 50 measurements).

\subsection{Results and discussion.}

Because gain values depend critically on calibration, but the contrast is largely independent from that, only the latter is used. The results are given in figures 5-1 and 5-2. Figure 5-1 shows the mean contrast \pm the standard error per frequency. A positive value means that the subjects performed better (i.e., had a higher gain) during the stimulation of visuo-vestibular interaction with an asynchronously moving target (mtVVI) than during the stimulation of visuo-vestibular interaction with an earth-fixed target (eftVVI). The fact that, on average, the subjects accomplished slightly better results in case of the more complex task of viewing an independently moving target than in case of the simple task with an earth-fixed target, is unex-

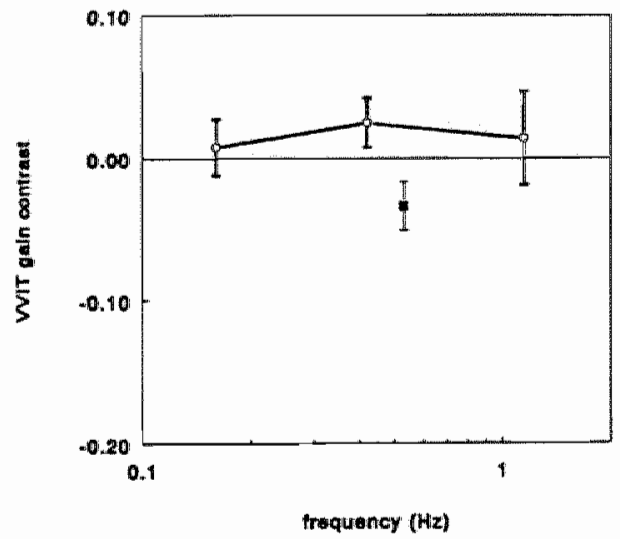

Fig. 5-1. Mean standard error of the relative difference of gains resulting from miVVl and eftVWl stimulation, depending on stimuius frequency.

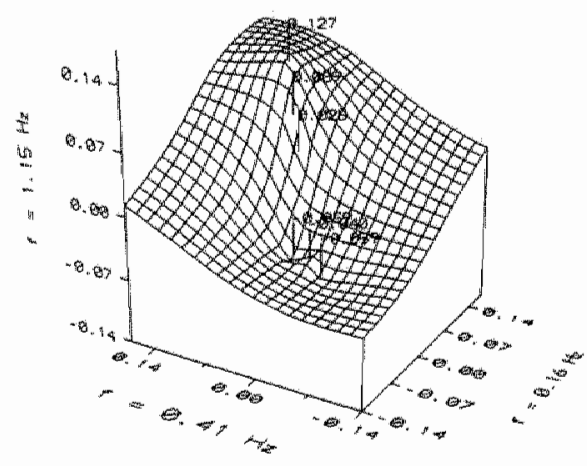

Fig 5-2 Multivariate plot of gain contrast responses. The univariate limits enclose the 95 m probability' standard reference ranges. 


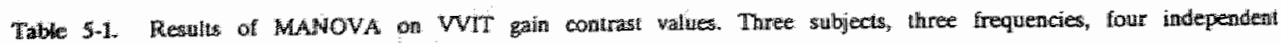
measurements: per subitect. - requency combinalion.

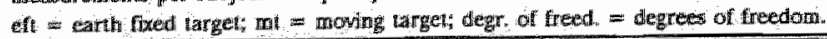

\begin{tabular}{|c|c|c|c|c|}
\hline \multirow{2}{*}{$\begin{array}{l}\text { llest } \\
\text { ansialydis }\end{array}$} & \multirow{2}{*}{ 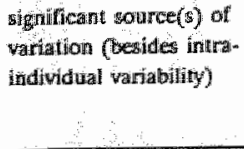 } & \multirow[t]{2}{*}{ mean } & \multicolumn{2}{|c|}{ wartance } \\
\hline & & & $\begin{array}{l}\text { intran. } \\
\text { individiual } \\
\text { (defer. of freed.) }\end{array}$ & $\begin{array}{l}\text { inter- } \\
\text { tricliwitual } \\
\text { (degr. of freed } 2 \text { ) }\end{array}$ \\
\hline $\begin{array}{l}\text { gatin } \\
\text { contraist }\end{array}$ & none & $-26.4 \times 10^{-3}$ & $\begin{array}{l}194 \times 10^{3} \\
(31)\end{array}$ & $0.909 \times 10^{3}$ \\
\hline
\end{tabular}

pected. If it is not due to chance (which it probably is, because it does not differ significantly from zero at the $5 \%$ level), then perhaps it might be explained by the mtVVI stimulation being less boring than the eftVVI stimulation.

Gain contrast is immune for both errors in calibration and spurious phase shifts introduced by the measurement equipment, and therefore the different responses to the chair-delivered and the purely visually delivered frequencies in figure 5-1 might be the best evidence in favor of the hypothesis that $\mathrm{mtVVI}$ is more than the sum of SP and visual VOR suppression. However, this difference might well be caused by the effect of prediction. The gain contrast for the chair-delivered frequencies is calculated from the response to sum-of-4sines stimulation compared to that to sum-of-3sines stimulation. But the gain contrast for the visually delivered frequency results from comparing responses to sum-of-4sines stimulation to that to single-sine stimulation. Single-sine visual stimulation allows full prediction, while the mtVVI stimulation may do so less or not at all. This could also explain the negative contrast at $0.53 \mathrm{~Hz}$.

Figure 5.2 shows the individual gain contrast values plotted in a perspectivic way for the three chair-delivered frequencies, using the limits of the univariate reference intervals as will be given below in table 5-2. Obviously, a high correlation can be expected between the responses to the various frequencies per subject, and this is

Tathe 5-2 Lower and wppet limits, defining nomal WVIT responses. Boid printing guides choosing between frequency-specific and frequency-averaged limits acconding 10 MANOVA established significance of stimulus frequency. Surpassing the parenthexed limits probably does nou indicate a pathological condition.

\begin{tabular}{|c|c|c|c|c|c|c|}
\hline & \multicolumn{4}{|c|}{ chair-dielivered frequencies } & \multirow{2}{*}{$\frac{\text { visual }}{0.53 \mathrm{~Hz}}$} & \\
\hline & $0.16 \mathrm{~Hz}$ & $0.41 \mathrm{H}$ & 1.15 $\mathrm{Hz}$ & $\begin{array}{l}\text { froguency- } \\
\text { averaged }\end{array}$ & & \\
\hline \multirow[t]{2}{*}{ VVII gain contrast } & -0.125 & -0.090 & +0.210 & -0.140 & -0.150 & lowwer limit \\
\hline & $(0.140)$ & $(0.140)$ & $(0.235)$ & $(0.175)$ & $(0.085)$ & upper limit \\
\hline
\end{tabular}


confirmed here. This figure vividly illustrates the need to analyze the variances of controls and groups of patients in a multivariate way. And further, it shows that using only the univariate limits for diagnosis would be very conservative if some pathology would diminish this frequency-correlation.

Table 5-1 gives the results of analysis of variance with a repeated measurements design. It is found that the intra-individual variations dominated the responses, and that subject or frequency did not influence results significantly. This allows one mean response to be calculated.

A reference range is calculated in such a way, that an independent, next measurement of a normal subject would have a chance of $95 \%$ to fall within that interval. The resulting lower and upper limits are given in table 5-2. Because the frequency had much less effect than the intra-individual variations, the frequency-averaged value should be used, and it is printed in bold to emphasize this. Further, there is no obvious reason why the contrast values should not exceed some positive value (if subjects perform much better at the more difficult task than at the easier task, one could suspect a change in alertness, rather than some pathology), and so those upper limits are printed within parentheses. If gains in response to mtVVI stimulation far exceed 1.10 , however, it may be pathological, nevertheless. It is hard to imagine that such a de-inhibition can be triggered by making the visual target move, but because only our gain contrast is reliable, and not the separate gain values, we can not make any definitive statements on this matter.

\subsection{Appendix}

To calculate a relative difference, the following formula can be used:

$$
\begin{aligned}
& \Delta=\frac{x_{1}-x_{2}}{x_{1}+x_{2}} \\
& x_{1}, x_{2} \text { values to be compared } \\
& \triangle \quad \text { relative difference }
\end{aligned}
$$

which is one of the contrast functions.

This relative difference combines the attractive properties of two other ways of comparing values, while it does not have their drawbacks. Taking the difference has the advantage of summational symmetry (i.e., $(\mathrm{a}+\delta)-\mathrm{a}=-(\mathrm{a}-(\mathrm{a}+\delta)))$, but has as a disadvantage that it remains sensitive to calibrational errors (i.e., $(c+\epsilon) \cdot(a+\delta)$ $(c+\epsilon) \cdot a=(c+\epsilon) \cdot \delta)$. Conversely, taking the ratio is immune for calibrational errors $((\mathrm{c}+\epsilon) \cdot(\mathrm{a}+\delta) /(\mathrm{c}+\epsilon) \cdot \mathrm{a}=(\mathrm{a}+\delta) / \mathrm{a})$, but has no summational symmetry $((\mathrm{a}+\delta) / \mathrm{a} \neq$ $-a /(a+\delta))$. This latter disadvantage can be remedied by taking the logarithm, which 
makes the result hard to interpret, however. A relative difference as given above, is independent of calibration, because any constant factor is removed by the division, and it is summationally symmetric because of the subtraction.

Of course, some data (like phase shifts) are unaffected by calibrational errors, but are sensitive to errors in offset, requiring nothing else but subtraction. 


\section{Effect of asynchronous visual stimulation on the Vestibulo-Ocular Reflex phase shift of normals.}

\subsection{Abstract.}

A vestibular function test (named Visuo-Vestibular Interaction Test: VVIT) is presented, in which a visual target may move asynchronousty with a pseudio-randomly oscillating rotatory chair.

Here, normative data are given for the phase shift, while the gain and the coherence are considered elsewhere (chapters 5 and 7, respectively). Attention is drawn to the artificial phase shifts introduced by the analog-to-digital converter and other hardware, which might explain the observed shifts in responses to single-sine stimuli. The found phase-lead in case of sum-of-sine stimulation with an earth-fixed, as well as with an asynchronously moving visual target is discussed in relation to that reported for suppression of the VOR by visual fixation and for altering the VOR by imagining a visual target.

\subsection{Introduction.}

A full introduction of the VVIT was given earlier (chapter 5), so here only the essentials will be repeated.

In order to elude the variation inherent to measuring the vestibulo-ocular reflex (VOR) in the dark, a visual target is presented. The usual ways in which the resulting visuo-vestibular interaction (VVI) is studied, (namely, by offering either an earth-fixed target or a chair-fixed target) allow, but may not require, the two systems to interact. To force an interaction between smooth pursuit (SP) and VOR, we use a visual target which moves asynchronously with the chair. Predictive effects are reduced by having the chair move in a pseudo random (sum-of-sines) way. As both visual target movement and chair movement is below the SP upper limit, we expect normals and patients to perform equally well to either one of thern. But because the combination is above the SP upper limit, we expect patients to perform worse than normals to the combined stimulus, assuming that they will overburden their SP system in an effort to compensate for the insufficient vestibular fucntion. By subtracting the visuo-vestibular interaction with an earth-fixed target (eftVVI; i.e., visually enhanced VOR) stimulus and the response to it, from the visuo-vestibular interaction with an asynchronously moving target (mtVVI) stimulus and the response to it, the visual components are obtained. It is hypothesized that the vestibular function is reflected by the ratio of the smooth and saccadic eye movements (and thus by the gain of the median-filtered response). Different phase shifts for normals and patients and for eftVVI and miVVI 
might be expected in relation to the complexity of the stimulus and the type of neural processing it is subjected to.

For the following review, the frequency range is divided in low ( $<1 \mathrm{~Hz}$ ), medium $(1<\mathrm{f}<3 \mathrm{~Hz}$ ), and high ( $\mathrm{f}>3 \mathrm{~Hz}$ ), and the type of signal in single-sine, and pseudo-random.

In general, eftVVI responses are reported to have only small phase shifts.

For single-sine eftVVI stimulation at the low frequencies, Baloh et al. (1984) found phase shifts around zero, as did Furst et al. (1987) and Möller et al. (1990), who found a little more small phase leads, and Kasteel-van Linge \& Maas (1990), who found a little more smal] phase lags. At the medium frequencies and/or the high frequencies, Larsby et al. (1984), Hine \& Thorne (1987), and Kasteel-van Linge \& Maas (1990) found small phase lags. Keller (1978) found with monkeys a persistent phase lead of 3 degrees for both dVOR and eftVVI stimulation at frequencies from $0.5 \mathrm{~Hz}$ to $4.0 \mathrm{~Hz}$.

For sum-of-sines eftVVI stimulation, Furst et al. (1987) found small phase leads at the low frequencies.

And for eftVVI stimulation with an unspecified type of pseudo-randomness, Larsby et al. (1984) found small phase lags at the medium frequencies, and larger phase lags at higher frequencies.

Except for Hine \& Thorn (1987), who attributed a $5^{\circ}$ phase lag to the electrooculography (EOG) low-pass filters, none commented on correcting for phase shifts introduced by the hardware (EOG filters, analog-to-digital ( $A D$ ) card).

\subsection{Material and methods. 6.3.1 Subjects.}

Six controls ( 3 males of 23,33, and 37 years, and 3 females of 23, 26, and 34 years) were recruited among ourselves and our students, resulting in a lower median age ( 30 years) than that of the usual patient population for our clinic ( 45 years).

None of the subjects had any history of vestibular problems, and none of them had any difficulty with visually guided eye movements. All subjects, even those usely wearing glasses, could locate the visual target without any optical correction, and were therefore tested that way.

Three of them (two males of 23 and 37 years, and one female of 26 years) underwent the VVIT each at four different days in order to asses intra- and inter-subject variability. 


\subsubsection{Experimental set-up.}

The experimental set-up was as reported previously (chapter 5), and so only a brief description follows here. Subjects sat in a rotary chair moving in a pseudo random way (sum-of-sines: $0.16,0.41$, and $1.15 \mathrm{~Hz}$, each with a maximum velocity of $10 \% \mathrm{~s}$ ), while watching a dot which was either earth-fixed or moving independently $(0.53 \mathrm{~Hz}$, with a maximum velocity of $32 \%$ ). The eye position was measured using infra red reflection, and the head velocity was monitored with an angular rate sensor. Eye position, head velocity, and visual target position were sampled at a $50 \mathrm{~Hz}$ frequency for 125 seconds by a 12 bit analog-to-digital $(\mathrm{AD})$ converter (the first two after being filtered analogously with a cut-off frequency of $10 \mathrm{~Hz}$ ) and were digitally stored for offline analysis.

\subsubsection{Digital signal analysis.}

Processing and analysis of the signals was as reported before (see chapter 5). In summary, position data were digitally differentiated to velocities, after which the total stimulus was combined from visual target movement and chair movement. In order to calculate the phase shift over the same time length as gain and coherence were, the digitally stored signal was resampled by taking every third data point, so that the total time length fitted in the 2048 points available for fast Fourier transformation (FFT). The resulting lower sampling rate brought as an additional advantage a higher frequency resolution (at the cost of a lower frequency maximum, which was unimportant for the VVIT). Fast components (i.c., forward saccades, eye blink artefacts) in the response signals were removed or attenuated by using a median filter. The resampling made a different width of the median filter optimal than was used for the gain and coherence calculations, because the filter effect depends on number of data points as well as on time length. The used width was 0.35 s (nearest higher odd number of resampled data points is 7 for a recording time of $125 \mathrm{~s}$ ). The phase shift values at the response frequencies were extracted automatically from the spectrum, using preset criteria. 


\subsubsection{Statistical analysis.}

The statistical analysis was as described earlier (chapter 5). In brief, responses were analyzed for stimulus frequency, subject, and the interaction between frequency and subject, by analysis of variance. The limits of the reference intervals were calculated in the standard way, so that a next, independent measurement of a normal subject has 95\% chance to fall within this range. These ranges are equivalent to the ones advocated by Royston \& Matthews (1991) with error probabilities of only $0.55 \%$ and $0.83 \%$.

\subsection{Results and discussion.}

A striking feature of our VVI data is the different phase behavior of purely visually induced eye movements and visuo-vestibular induced ones. In figure $6-1$, the phase shifts (mean \pm standard error) of single-sine SP responses are compared to those of sum-of-sines eftVVI responses. While the SP response has a phase lag, the eftVVI responses have phase leads, increasing with frequency. More impressive, the same results are obtained when delivering all four frequencies simultaneously using a mtVVI paradigm (figure 6-2). Figures 6-3 and 6-4 show the individually calculated phase shift differences between mtVVI and eftVVI responses. The first figure of this pair shows the mean \pm the standard error per chair-delivered frequency, and the

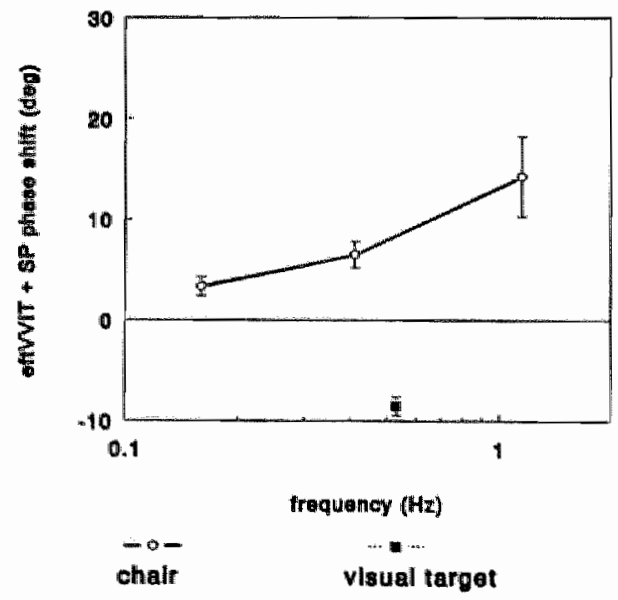

Fing 6-1. Uncorrected phise shifts for smooth pursuit ( $=$ visual target) and efrVVTr $(0=$ chair $)$. The single-sine smooth pursulit was conducted separately from the sum-ofsines eft VV I stimulation.

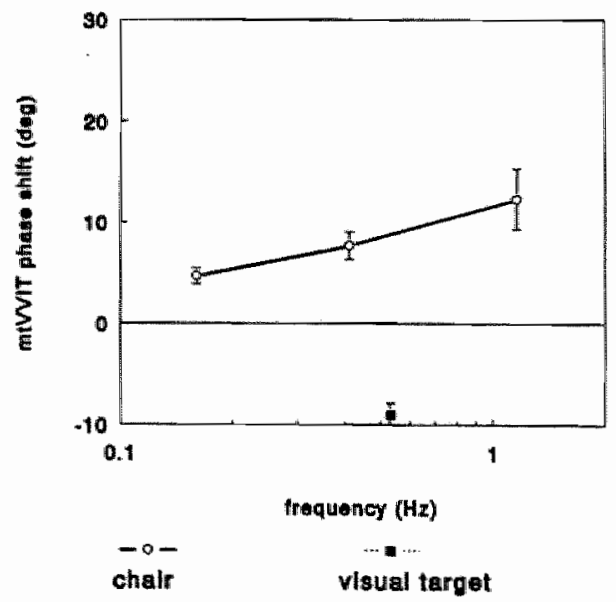

Fig. 6-2. Uncorrected pivase shifts for vistuo-westibuiary induced eye mowements $(O=$ chair) and visually induced ones ( = visual larget). All four frequencies were deliwer. ed simultanously by mVVI stimulation. 


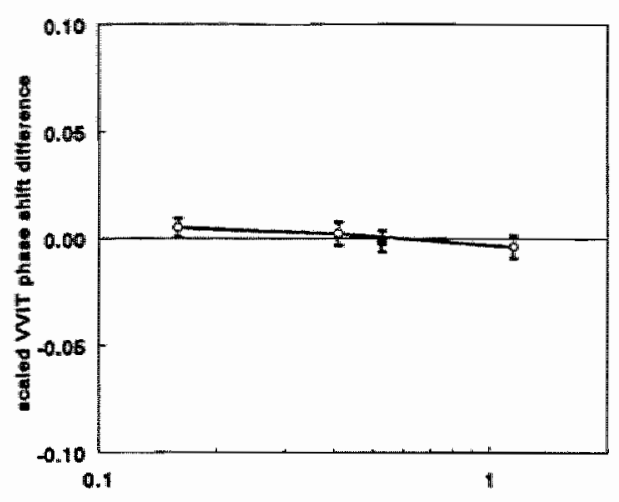

fruquemoy (Hz)

Fig 6-3. Scaled phase shift difference depending on stimulus frequency. $\theta=$ chairmdelivered trequencies, 1 purely visually delinered frequency.

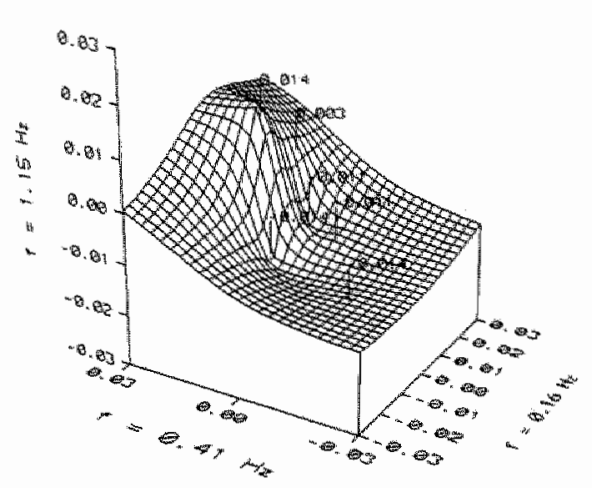

Fig. 64. Multivariate plot of scaled phase shift differences. The univariate limits enclose the $95 \%$ probability ranges.

second shows the individual values plotted in a perspectivic way for the three chairdelivered frequencies, using the limits of the univariate reference intervals as will be given below in table 6-2. Just as for the gain contrast, the expected correlation between individual responses to the various frequencies, is here confirmed.

The phase leads of eftVVI responses is in accordance with the results of Keller (1978) with trained monkeys, and with the results of Furst et al. (1987), but is in contrast with results obtained by different groups associated with the Linköping university (e.g., Hydén et al., 1982; Larsby et al., 1984). Interestingly, a similar phase lead as ours did have been found by most researchers for VOR suppression (caused by looking at a chair-fixed visual target: cftVVI). We have not yet gathered cftVVI data, but it would be interesting to see whether their phase shifts really would be similar to those of the current eftVVI and mtVVI data, as we expect them to be.

Because in the literature there is no unequivocal support for our eftVVI phase leads (reports on $\mathrm{mtVVI}$ stimulation were not found), because their frequency-dependency is opposite to the one for the VOR in the dark (dVOR), and because a prediction effect seemed unlikely, doubt arose in how far it is real. At first thought, one may think of some accidental signal inversion which might explain the opposite phase shifts. But signal inversion introduces a $180^{\circ}$ shift and does not alter the sign while leaving the magnitude unchanged. Next, signal channels might have been interchanged, but the fact that during mtVVI stimulation there is a phase lead at the frequencies delivered by chair movement and at the same time a phase lag at the frequency delivered by the visual target movement, eliminates this possibility. 


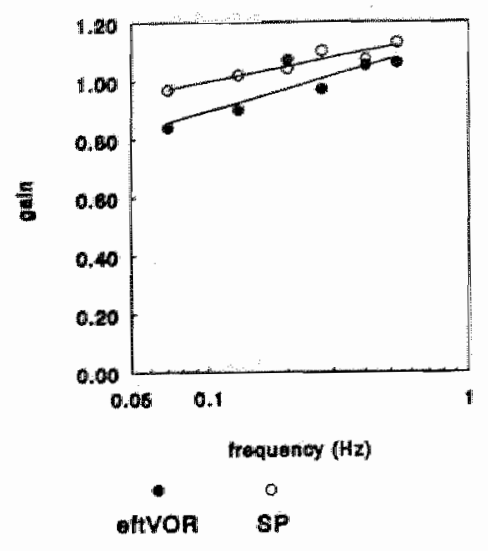

Fig 6-5. Gain valties of one subject for Vestibulo-Ocular Reflex with earth-fixed targer (- = eftVoR) and for Smooth Pursuit $(O=$ SP). The difference berween the wo should be interpreted with some caution. (Sarse data analyzed as in figure 6-6).

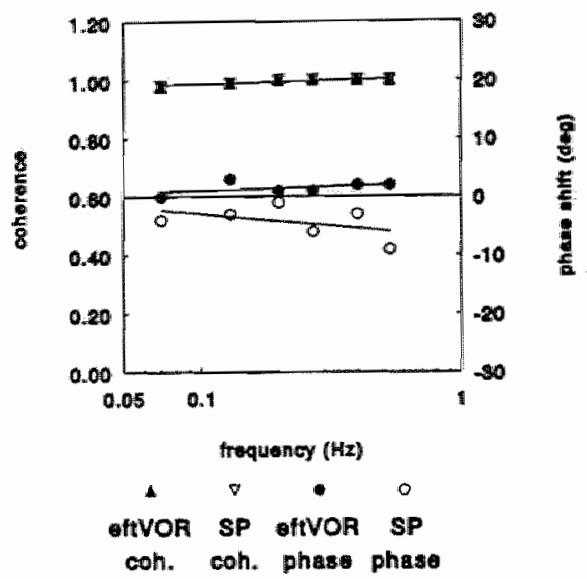

Fip 6-6. Coherence and phase shift walues of one subject for Vestibulo-Ocular Reflex with earth-fixed target ( 4 respectively - efiVOR) and for Smooh Pursuit ( $\nabla_{1}$ respectively $0=S P$ ). (Same data analyzed as in figure $6 \mathrm{~s}^{5}$ ).

Therefore, this matter was investigated further.

For dVOR, phase leads occur at frequencies below $0.2 \mathrm{~Hz}$ (Baloh et al., 1984), and, in accordance with the first order transfer function, they increase with decreasing frequency. So our phase lead obviously is not a characteristic of the direct vestibular reflex. Prediction seemed unlikely, because the phase lead occurred for all three, simultaneously applied, frequencies, while for sum-of-sines visual stimuli, prediction optimizes the highest frequency response only (Barnes et al, 1987, and Barnes \& Ruddock, 1989; the gain is restored to the single-sine level for the highest frequency only, if this exceeds $0.8 \mathrm{~Hz}$ ). Furthermore, one might expect the Vestibulo-Ocular Reflex with earth-fixed target (eftVOR) to behave in the same way during the VisuoVestibular Interaction Test with earth-fixed target (eftVVIT; whole-body movement of the subject) and the Vestibular Autorotation Test with earth-fixed target (eftVAT; stationary subject shakes his head), as both tests involve viewing a stationary target while the head is moving. In general, however, analysis showed a phase lead in case of the eftVVIT, but a phase lag in case of the eftVAT. The latter is also reported by O'Leary et al. $(1988,1990)$, who found a phase lag of $6^{\circ}$ at $2 \mathrm{~Hz}$ and of $8^{\circ}$ at $3 \mathrm{~Hz}$. One of the differences between the two methods in our laboratory, is the way eye position is measured. The VVIT is done using the IRIS measuring device at one PC system, while the VAT is done using ENG electrodes at another PC system. Another difference is the nature of the head movement. During the VAT, the head moves approximately sinusoidally, while during the VVIT the head moves in a pseudo- 
random way.

To check whether the difference in phase behavior can be explained by the degree of randomness of the head movement, the eftVOR and the Smooth Pursuit (SP) were measured for single-sine stimuli of various frequencies. To prevent a time effect from causing a difference between the results of the two paradigms, they were interleaved, ie., the measurements using chair movement and those using target movement, alternated. Furthermore, stimulation was done starting with the higher frequencies and ending with the lower ones, so that departure from zero phase shift could not be attributed to tiredness of the subject. Figures 6-5 and 6-6 show the results. The gain is increasing with frequency for both eftVOR and SP (figure 6-5), and at every frequency but one the SP gain exceeds the eftVOR gain. This latter may be caused by the calibration problems we have, and so the gains might be similar, or it may reflect an increased use of forward saccades in case of the eftVOR. The coherence is identical for both paradigms (figure 6-6). The qualitative difference found at the VVIT, using sum-of-sines stimulation, are found here too, using single-sine stimuli (figure 7-4), but the magnitudes of the single-sine eftVOR phase leads, however, are much less than when using a sum-of-sines stimulus at a VVIT. So predictability changes phase shifts towards zero, but it does not reverse them.

The single-sine eftVOR phase lead is very small. Expressed as time, it is less than our sample period, but it is found consistently, nevertheless. (A phase shift of less then the sample period can be found, because it is the average of the phase shifts of a great number of time samples from a signal that contains also noise). Given the frequency-dependent increase of the phase lead, checking should have been done with the highest possible frequency, but unfortunately new measurements were then not possible because of bugs in a new version of the chair operating software.

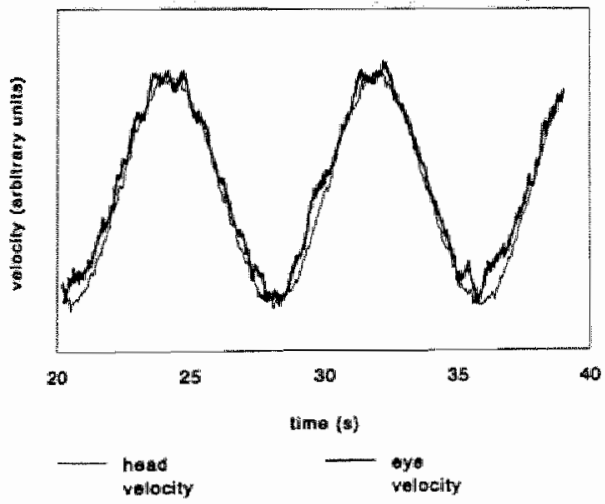

Figure 6-7. Result of eftVOR at $0.13 \mathrm{~Hz}$ shows a smali phase lead of the eyes with respect to the head.

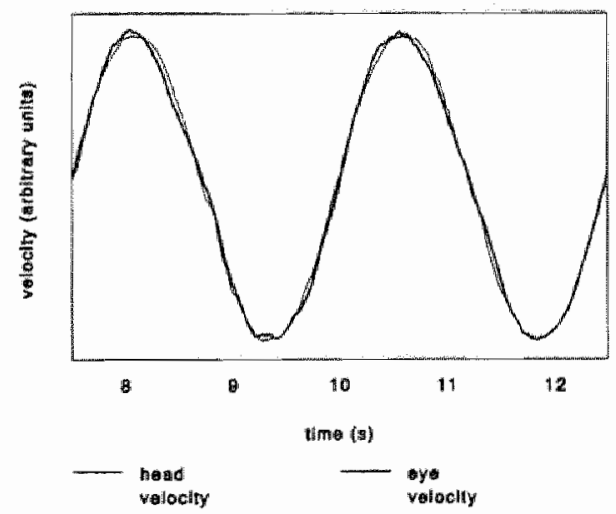

Finure 68. Resull of eftWOR at $0.4 \mathrm{~Hz}$ shows a small phase lead of the eyes with respect to the head. 


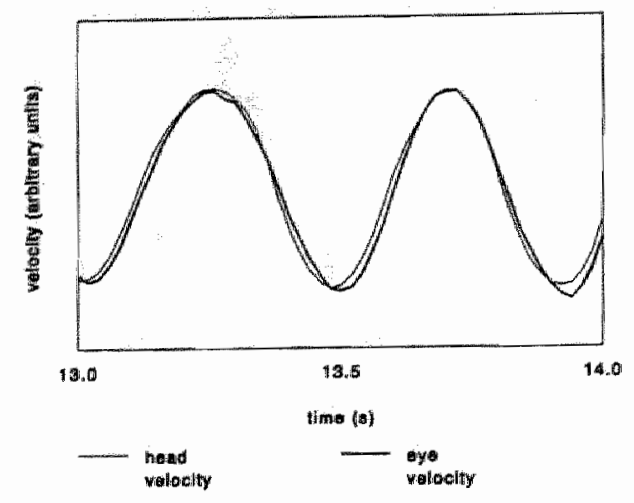

Figure 6-9. VAT resull shows a phase lag of the eyes with respect to the head.

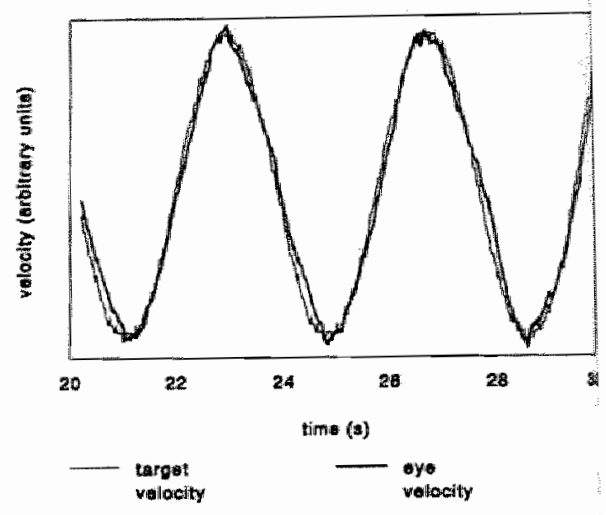

Figare 6-10. SP resull shows a small phase lag of the esen with respect to the target.

When the absolute value of a phase shift increases with frequency, it might be caused by introducing a fixed time shift during analysis, e.g., by approximating differentiation by differencing time samples. Because of the opposite shifts of SP and eftVOR, and because the frequency-dependency varies with stimulus predictability, it is deemed unlikely that the observed phase shift behavior is due to analysis.

To check whether the observed differences in phase behavior of the VVIT and the VAT was caused by the method of gathering data, a VAT was repeated with the same equipment as used for the VVIT. Phase lags were found for this IRIS-monitored VAT, just as for the electrodes-monitored VAT's, so the different ways in which the data was collected, was thought to be no explanation.

Also the analyzing software was checked by inspecting some of the raw data. Figures 6-7 and 6-8 display parts of the almost ${ }^{1}$ original data on which figures 6-5 and 6-6 are based, and they show small phase leads for the eftVOR (at: 0.13 and $0.40 \mathrm{~Hz}$ ). Similarly, figures 6-9 and 6-10 show phase lags for the VAT $(f=2.1 \mathrm{~Hz})$ and the SP $(f=0.27 \mathrm{~Hz})$, respectively.

Finally, data were corrected for the various time shifts, introduced by components of the stimulating, detecting and registrating system (figure 6-11). The fact that the $\mathrm{AD}$ card has only one $A D$ converter that scans the various channels sequentially, instead of simultanously, introduces a time shift that depends on how far the stimulus and response channels are apart, and on the sample frequency (see appendix).

The low-pass filters introduce a lag, that theoretically should be about $16 \mathrm{~ms}$. Butt

\footnotetext{
The original eye position dan was differentiated (to obtain eye velocity) in such a way, that it could easily be veriated that no phase shifts were introduced here (differentiation in a spreadsheet, to prechude errors in loop counters).
} 


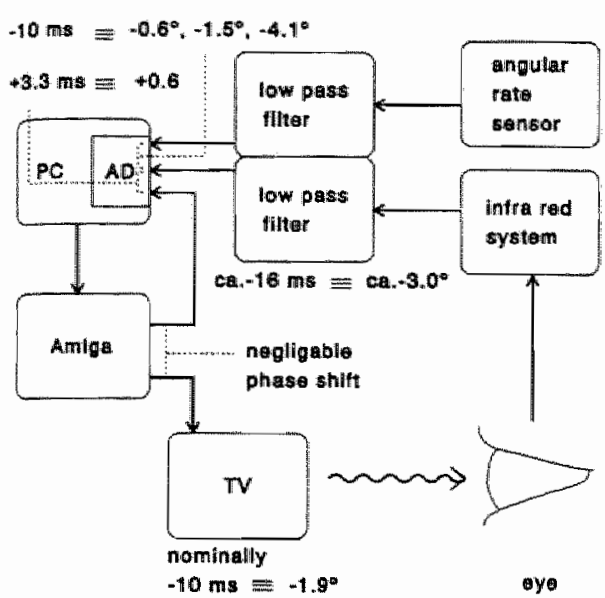

Fig. 6-11. Relative time shifts, introdived by measuremen! comipoarents. The equivallent phase shifts are shown for the purely vistally delivered stimulus frequency, and for the chairdelivered: frequencies.

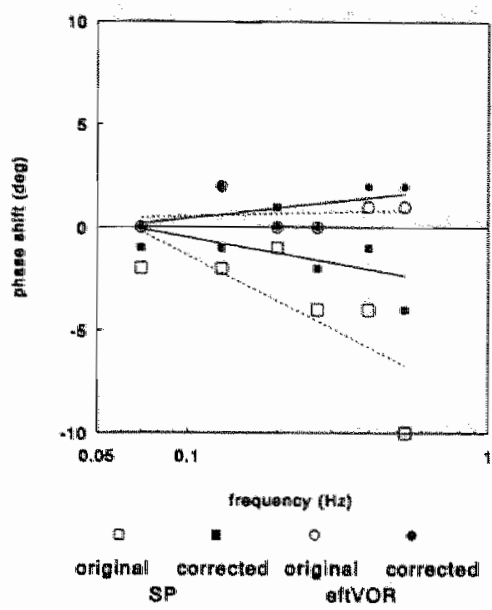

Fig. 6-12 Comparison of original (broken lines) and corrected (continuous lines) phase shifs, resultung from single-sine SP and efWWI stmulation. Correction is based on known time shifts of the $A D$ card, the filters, and the $T$.

being built of analog components deviating randomly from their specifications, their actual delays may differ from this. By applying identical inputs to various channels, it was found that the delays caused by the combinations of filters and amplifiers could differ between channels by as much as $6 \mathrm{~ms}$.

In figure $6-12$, the original and the corrected phase shifts are depicted for the singlesine SP and eftVVI, together with the results of linear regression. The responses at the purely visually delivered frequency were corrected for the time shifts introduced by the television set (nominally lagging half the refreshment period, i.e, $10 \mathrm{~ms}$ ), the low-pass filter (a first-order filter with a cut-off frequency of $10 \mathrm{~Hz}$, lagging $16 \mathrm{~ms}$ ) and the $\mathrm{AD}$ card (advancing $3.3 \mathrm{~ms}$ ), totaling $-22.7 \mathrm{~ms}$. The responses at the chairdelivered frequencies were corrected for the $\mathrm{AD}$ card (lagging $10 \mathrm{~ms}$ ) and the difference between the low-pass filters for the eye signal and the angular rate sensor signal (advancing $6 \mathrm{~ms}$ ). (Both detectors (angular rate sensor and infra red reflection device) are assumed to have similar latencies, which are thought to be small compared to those of the filters and the AD card). The lines fitted to the corrected phase shifts are conform the expectations: no phase shift for the low frequencies, and for the middle frequencies phase lags for the SP and phase leads (as expected from the pseudo-random stimulation) for the eftVVI, which are increasing with frequency. Alternatively, one may assume that single-sine stimulation would allow perfect prediction, and then the frequency-dependent increases might indicate hardwareintroduced delays that are not not yet accounted for, or they may be random fluctuations. This will be investigated in future research. 


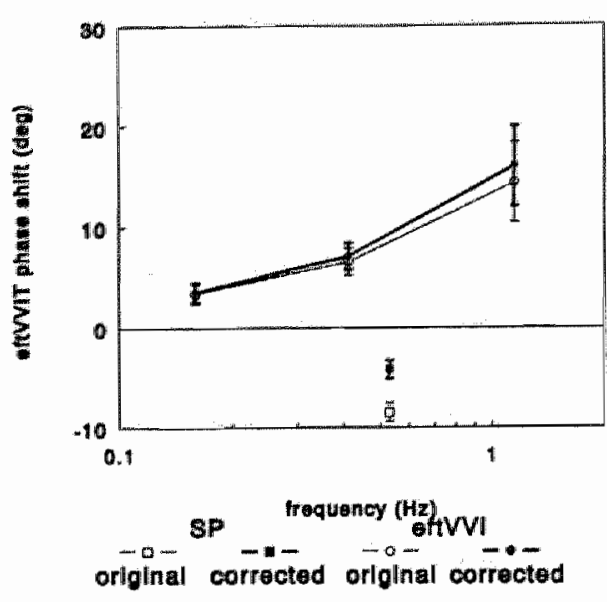

Fig 6-133. Lncorrected and corrected phase shifis comparted. Responses to sum of -sine eftWh stimulation were corrected for knowis hardwaremintroduced phase sibifits.

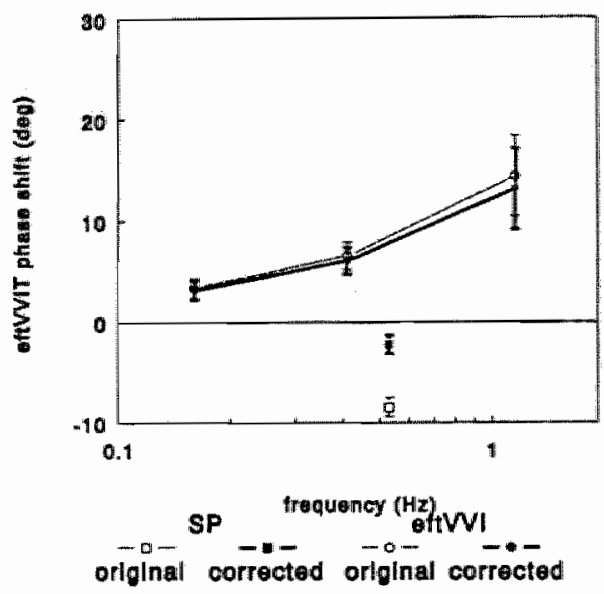

Fig 6-14. Uncorrected and corrected phase shifis cortw pared. Responses tho sum of sime efiVVI stimulation wer corrected, using time shifts: derived from single-sine meas urements.

In figures 6-13 and 6-14 the corrected phase shifts are compared with the uncorrected ones for sum-of-sines eftVVI stimulation and single-sine SP stimulation for the means of the six controls. In figure $6-13$, correction is based on the known time shifts of the components, as mentioned above and as given in figure 6-11. In figure 6-14, however, correction is based on the assumption that for low and middle frequency single-sine stimulation the biological phase shift should be zero (due to a predictor). The deviations seen in figure 6-6 are attributed to measurement artefacts, of which not all sources are known. A trimmed mean time shift was calculated using the four middle values and excluding the highest and the lowest one. This resulted in a time shift of $-33 \mathrm{~ms}$ for the SP signal (as opposed to the components-derived value of $-22.7 \mathrm{~ms}$ ), and a time shift of $+3 \mathrm{~ms}$ for the eftVVI signal (as opposed to the componentsderived value of $-4 \mathrm{~ms}$ ). The fact that after this correction stil] clear phase leads remain, indicates that these phase leads are no artefacts, but that they most probably truly result from the subjects (who supposedly are no longer able to predict the stimulus because of the sum-of-sine pseudo-randomness).

In hindsight, it was clear that the opposite phase shifts of the original single-sine eftVVI and VAT measurements were caused by the opposite directions in which the $\mathrm{AD}$ cards of the two pieces of equipment scanned the channels. The Burr-Brown PCI-20000 (Burr-Brown Corporation, Tucson, Arizona, USA) used in the VVTT equipment scans from lower to higher channel numbers, while the $A D$ card in the Nystagliner (Jaeger/Tönnies, Würzbürg, Germany) used for VAT measurements scans from high to low. But it was still obscure why the VAT that was later repeated 
Table 6-L. Results of MANOVA on VWT phase sthift analysis results (corrected for artificial phase shits). Three subjects; three trequencies, four independent messurements per subject - freguency combination.

eff $=$ earth fued target; $\mathrm{mt}=$ moving target degr. of freed $=$ degrees of fredom.

\begin{tabular}{|c|c|c|c|c|c|}
\hline \multirow{2}{*}{$\begin{array}{l}\text { testi } \\
\text { analysis }\end{array}$} & \multirow{2}{*}{$\begin{array}{l}\text { signifficant source(s) af } \\
\text { variation (beside intra. } \\
\text { individual variability) }\end{array}$} & \multicolumn{2}{|l|}{$\operatorname{mean}(s)$} & \multicolumn{2}{|c|}{ सwriance } \\
\hline & & $\begin{array}{l}\text { subjic } \\
\text { subjiz } \\
\text { sutbi3 }\end{array}$ & $\begin{array}{lll}* & \cdots \\
* & \cdots \\
* & . . \\
\end{array}$ & 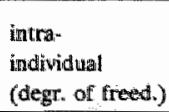 & $\begin{array}{l}\text { inter: } \\
\text { individual } \\
\text { (degr. of freed }=2 \text { ) }\end{array}$ \\
\hline $\begin{array}{l}\text { eff phase } \\
\text { shift }\end{array}$ & frequency $(0<0.0005)$ & 25 & 13 & $\begin{array}{l}478 \\
(31)\end{array}$ & 726 \\
\hline $\begin{array}{l}\text { wh puase } \\
\text { shift }\end{array}$ & $\begin{array}{l}\text { subject - frequency inter- } \\
\text { action }(p<0.01) \\
\text { frequency } \quad(p<0.025)\end{array}$ & $\begin{array}{cc}3 & 6 \\
4 & 11 \\
5 & 6\end{array}$ & $\begin{array}{c}5 \\
25 \\
14\end{array}$ & $\begin{array}{l}21.0 \\
(27)\end{array}$ & 210 \\
\hline $\begin{array}{l}\text { phase shift } \\
\text { difference }\end{array}$ & $\begin{array}{l}\text { inter-indrividual variation } \\
(p<0.025)\end{array}$ & $\begin{array}{r}0.5 \times 10^{3} \\
17.1 \times 10^{2} \\
3.2 \times 10^{3}\end{array}$ & & $\begin{array}{l}0.310 \times 10^{3} \\
(31)\end{array}$ & $1.41 \times 10^{-3}$ \\
\hline
\end{tabular}

with the VVIT equipment, showed phase lags comparable to those of the original VAT's, and not phase leads like the VVIT's. Then it was recalled that after the measurements for this study were completed, and before the checking of the VAT was carried out, the filters and the amplifiers had been reconnected in a different way. And so it is quite probable that this, in combination with the known dissimilar time lags, has given a second inversion. So comparable phase lags were obtained, probably because of the unfortuitous combination of two reversions.

Table 6-2 Lower and upper limits, defining normal VVIT responses. Bold printing guides choosing between freciluency-spedific and frequency-averaged limits, acconding to MANOVArestablished significance of stimalls frequency.

\begin{tabular}{|c|c|c|c|c|c|c|}
\hline & \multicolumn{4}{|c|}{ chair-delivered firequencies } & \multirow{2}{*}{$\frac{\text { vesulal }}{0.53 \mathrm{H} H 2}$} & \\
\hline & $0.16 \mathrm{~Hz}$ & $0.4 \mathrm{~Hz}$ & $1.15 \mathrm{~Hz}$ & $\begin{array}{l}\text { frequengy- } \\
\text { averaged }\end{array}$ & & \\
\hline \multirow{2}{*}{$\begin{array}{l}\text { mivVT phase shift } \\
\text { (corrected for known phase } \\
\text { shifts) }\end{array}$} & -1.0 & -20 & 85 & 40 & .13 .0 & lower limit \\
\hline & 11.0 & 185 & 36.5 & 22.0 & 4.0 & upper limit \\
\hline \multirow{2}{*}{$\begin{array}{l}\text { miVVIT phase shift } \\
\text { (corrected assuming single- } \\
\text { sine stimulation resillts in } \\
\text { zero phase shift) }\end{array}$} & -15 & -3.0 & -115 & -5.5 & -1.1 .0 & Lower linglt \\
\hline & 10.5 & 17.5 & 335 & 20.5 & 5.5 & upper limit \\
\hline \multirow[t]{2}{*}{ WTT phase shift difference } & .0 .020 & -0.030 & -0.035 & -0.030 & -0.036 & lower limit \\
\hline & 0.030 & 0.035 & 0.025 & $0.03 \times$ & 0.034 & upper lintait \\
\hline
\end{tabular}


Table 6-1 gives the results of analysis of variance with a repeated measurement design. Only the effect of the individual, of frequency and of their interaction is analyzed, because other studies had shown that age and sex do not influence responses to VVI stimulation significantly (see chapter' 9 "Effect of asynchronous visual stimulation on the VOR phase shift of patients, compared to that of normals'). It is found that during eftVVI stimulation, all three subjects responded in the same, frequency-specific way, leading to to three frequency-specific mean values. The subjects responded differently, however, to the mtVVI stimulation, and so those results may be averaged only per subject-frequency combination. By taking the individual differences, the variation due to frequency is removed, as responses to eftVVI and to mtVVI stimulation depend in the same way on frequency. This leaves the inter-individual variation and so subject-specific means can be calculated.

Two reference ranges are calculated in such a way, that an independent, next measurement of a normal subject would have a chance of $95 \%$ to fall within those intervals. The resulting lower and upper limits are given in table 6-2. No range for eftVVI responses is given, because MANOVA of results from controls and patients failed to find significant differences related to group designation (see chapter 9 "Effect of asynchronous visual stimulation on the VOR phase shift of patients, compared to that of normals"). Because in table 6-1 the frequency and the subjectfrequency interaction were found to influence results significantly in case of $\mathrm{mtVVI}$ stimulation, no frequency-averaging is allowed. The frequency-specific values are printed in bold to emphasize that these values should be refered to. Taking the individual differences removed the influence of frequency in table 6-1, and so here the frequency-averaged values should be used.

\subsection{Conclusion.}

It is concluced that the phase lead of VVI responses to a sum-of-sines stimulus is no artefact (neither from measurement, nor from analysis), while in case of single-sine stimulation it might be an artefact of data-acquisition.

Although several schemes can be brought up to explain the phase lead in case of mtVVI (e.g., a combination of VOR suppression and SP take-over, or that a mental representation contributes apparent target movement completely to an inappropriate VOR), those based on assuming some suppressional activity fail to explain the phase lead induced by eftVVI stimulation (i.e., visually enhanced VOR!). Based on the current data, it may be hypothesized that in the presence of a visual stimulus, the vestibular contribution is phase-advanced (actually, the original velocity information 
seems to be used, instead of being integrated first to obtain position information (figure 6 of Barr et al, 1976; Baloh et al, 1984); perhaps this is done in order to cancel the SP phase lag), while without VVI the simple pendulum model, as derived for the dVOR, holds. In case of single-sine stimulation, however, prediction appears to reduce the phase shift to zero, masking whether the eye movement is from visual or vestibular origin. In this line of reasoning, the phase lead in case of cftVVI is not indicative of suppression (of the VOR), but of vestibular information (used for VOR self-cancelation). The fact that imagining an earth-fixed visual target while being oscilated in the dark, results also in a phase lead (only when doing so increases the dVOR gain, i.e., for amplitudes less than $50^{\circ}$; Baloh et al., 1984), is consistent with this. That the phase leads $\left(1^{\circ} \ldots 3^{\circ}\right)$ found by Furst et al. (1987) for low-frequency sum-of-sines eftVVI stimulation are lower than ours, may be explained by their choice of the frequencies of the constituting sines, making their stimulus less random then the one used in the current study. By using $0.05,0.15$ and $0.35 \mathrm{~Hz}$, the repeat frequency of their stimulus is $0.05 \mathrm{~Hz}$, which is to say that at any time the stimulus is identical to the one 20 seconds before. In contrast, our choice of harmonically unrelated frequencies produced a stimulus with a repeat period much longer than the measurement period (three months versus two minutes, but a more realistic estimate is circa 30 minutes; see the appendix).

In disagreement with these phase leads, the in an unspecified way randomized stimulus used by Hydén et al. (1982) and Larsby et al. (1984), produced no phase shifts under eftVVI conditions, which would mean that their randomization had no effect compared to single-sine stimulation. The fact that the former group found zero phase shift for eftVVI stimulation when using chair velocity (instead of head velocity) to calculate phase shifts, while the latter group found also almost no phase shift when using head velocity, but now did found a phase lag when using chair velocity, suggests that those groups associated with the Linköping university might have an additional, unknown factor that influences phase. This is also suggested by the remarkable difference between cftVVI-induced phase shifts under single-sine and pseudo-random conditions (here randomization might have had an effect). Compared to single-sine stimulation, the phase lead with pseudo-random stimulation is increased (changed from negative to zero) at $0.25 \mathrm{~Hz}$, is decreased at $0.75 \mathrm{~Hz}$, and is the same at $1.5 \mathrm{~Hz}$ (but perhaps these differences are not statistically significant).

Our VAT-produced phase lags almost disappeared for normals by correcting for the shifts introduced by the hardware, bringing these results in line with those resulting from passive single-sine whole-body eftVVI stimulation. O'Leary (1988, 1990), however, found phase lags of $6^{\circ} . .8^{\circ}$, and so perhaps an explanation still is needed. The fact that VVIT results show phase leads while VAT results may show phase lags, might be explained by the notion that the VOR evolved to stabilize the retinal images 
against unintended head movements. Intended head movements, in contrast, are usually made in order to fixate a new target or to follow a moving target. In the first case no phase advancement of the VOR is wanted, because the VOR then does not work in conjunction with the phase lagging visual system in a feedback mode, but in an open loop mode with the ballistically operating saccades. And in the second case (head-free pursuit), no VOR at all is wanted. So if there really is a discrepancy between eftVVI results produced by passive (VVTT) and active (VAT) movement, this might stem from different demands made on the system under such circumstances. But, at the other hand, movements generated by the subject himself should be more predictable than forced movements, and phase shifts closer to zero then those obtained by single-sine eftVVI stimulation, could then be expected.

\subsection{Appendix}

Phase shift due to analog-to-digital conversion.

Because the analog-to-digital (AD) card has only one AD converter that scans the various channels sequentially (instead of several $A D$ converters sampling simultanously), a time shift is introduced that depends on how far the stimulus and response channels are apart, and on the sample frequency. The sample frequency determines the period within which all channels have to be read. This period is used completely, which means that the time consumed per channel is not fixed, but depends on that sample frequency. For the VVIT, we read 6 channels at a sample frequency of $50 \mathrm{~Hz}$, leading to a time shift of $3.33 \mathrm{~ms}$ per channel. Whether the apparent phase shift will be leading or lagging, depends on which channel is scanned first. When calculating phase shifts, all channels are assumed to be sampled at the same time. By doing so, a channel which is read later, is in effect artificially advanced in time (or, equivalently, an earlier read channel is artificially delayed). And so when the response signal is sampled after the stimulus signal, the calculated biological phase shift has a spurious leading component.

The phase shift introduced by the $\mathrm{AD}$ card is given by

$$
\varphi_{A D}(f)=\frac{\Delta \cdot f \cdot 360^{\circ}}{f_{s} \cdot n}
$$


$\Delta$ difference in channel number, related to scan direction:

$\Delta=\left(\mathrm{nr}_{\text {chem }}(\right.$ response $)-\mathrm{ni}_{\text {chum }}($ (stimulus $\left.)\right) \cdot \mathrm{dir}_{\mathrm{scon}}$

in which dir sew is plus unity for scanning from low to bigh, and minus unity for scanning from high to low

f measured frequency

$f_{s}$ sample frequency

$n$ number of channels, read by a single $\mathrm{AD}$ converter

For the highest frequency of the VVIT (i.e., $1.15 \mathrm{~Hz}$ ), this results in an artificial phase shift of $-4^{\circ}$.

In our latest set-up for the Vestibular Autorotation Test (VAT), we scan 7 channels from high to low, of which the first contains the eye position and the seventh the head velocity. Sampling is done at $100 \mathrm{~Hz}$. For the highest frequency that most normals can attain, namely $6 \mathrm{~Hz}$, the $\mathrm{AD}$ card introduced phase lead is then $18.5^{\circ}$.

Repeat period of sum-of-sines signal.

Finding the repeat frequency of a sum-of-sines stimulus consists of finding the smallest common multiple for the periods of the constituting sines. Because fractional values are involved, simple factorial decomposition is not possible, and the precision is of paramount influence.

A quick way to calculate the repeat period would be to turn the fractional values into integers by multiplying them with a fixed power of ten and afterwards dividing the found smallest common multiple by the same number. The results obtained in this way are given in table $5-3$, where it is assumed that period time is significant up till the second decimal (because of the DA card frequency of $100 \mathrm{~Hz}$, leading to a time resolution of $0.01 \mathrm{~s}$ ). The found period duration of more than three months seems to be exaggerated.

A more realistic estimate may be obtained by conceiving the possible errors in the constituting periods as tolerances. The problem then turns into finding for all periods

Table 5-3. Smallest common multiple as found by tactorial decomposition of the rounded valtues of 100 times the constituting periods. $T=$ period time.

\begin{tabular}{|l|l|l|l|}
\hline \hline & $T$ & round $(100 \times \mathrm{T}$ & factorial decomposition \\
\hline 0.16 & $6.2500 \ldots$ & 625 & $5^{4}$ \\
0.41 & $2.4390 \ldots$ & 244 & $2^{2} \times 61$ \\
0.53 & $1.8868 \ldots$ & $3^{3} \times 7$ \\
1.15 & $0.8696 \ldots$ & 87 & $3 \times 29$ \\
\hline \hline
\end{tabular}


Table 54. Humbering of frequencines and their accompanying period times will be used in table s.5. T $=$ period time

\begin{tabular}{|l|l|l|}
\hline number & Irequency & $\mathrm{T}$ \\
\hline 1 & 0.16 & $6.25 \pm 0.01$ \\
\hline 2 & 0.41 & $2.44 \pm 0.01$ \\
3 & 0.53 & $1.89 \pm 0.01$ \\
4 & 1.15 & $0.87 \pm 0.01$ \\
\hline
\end{tabular}

values within the tolerances and as close as possible to their nominal values, which would lead to a minimal common multiple. Because no method could be found to do this for all values simultaneously, pairs of values are considered. For a concise notation, the frequencies are numbered as given in table 5-4.

Of all pairs of frequencies, the smallest common multiple is sought that can be
s. From the resulting common multiples, made from the periods using their tolerances. From the resulting common multiples, one less than the used number of pairs is selected, using two criteria: $\underline{a}$ those with the highest relative tolerances are used, and $\underline{b}$ all members should be represented at least once. The second condition is obvious, while the first condition prefers period times that are closest to the nominal values (i.e., the available tolerance is least used). With our four frequencies (table 5-4) and with a DA card precision of 0.01 s (DA card frequency of $100 \mathrm{~Hz}$ ), the results of table 5-5 are obtained. The so found period of $1875 \mathrm{~s}$ ( $\approx 31$ minutes) is considerably smaller than the earlier found period of $8358525 \mathrm{~s}$ ( $\approx$ three months).

As a check, the actually used period times can be compared with the intended ones. Table 5-6 shows that the differences are all much smaller than the $0.01 \mathrm{~s}$ that is due to the DA card working frequency. This makes the 31 minutes a probable estimate of

Table 5-5. Smallest common multiples found per pair of periods. Numbering of frequencies is given in table $5-4$. Bold printed relative tolerances indicate results that are used for the next stage, using highesti relative tolerance and representavion of all used periods as criteria

\begin{tabular}{|c|c|l|}
\hline pair & smallesti common multiple & relative tolerance \\
\hline $1-2$ & $43.77 \pm 0.05$ & 0.0011 \\
$2-3$ & $17.04 \pm 0.03$ & 0.0018 \\
$3-4$ & $9.47 \pm 0.01$ & 0.0011 \\
$4-1$ & $25.00 \pm 0.04$ & 0.0016 \\
$1-3$ & $18.77 \pm 0.006$ & 0.0003 \\
$2-4$ & $12.20 \pm 0.05$ & 0.0041 \\
\hline$(2-4)-(2-3)$ & $85.32 \pm 0.13$ & 0.0015 \\
$(2-3)-(4-1)$ & $375.00 \pm 0.45$ & 0.0012 \\
$(4-1)-(2-4)$ & $425.73 \pm 0.13$ & 0.0003 \\
\hline$(2-4)+(2-3)-(2-3)-(4-1))$ & $1875.00 \pm 0.65$ & 0.000035 \\
\hline
\end{tabular}


Tabke 5-6. Period durations used to derive a smallest common muthiple of 1875, compared tho their nomirnal values, The

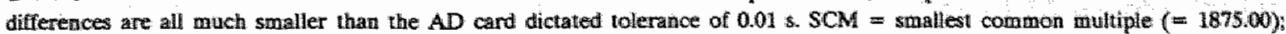

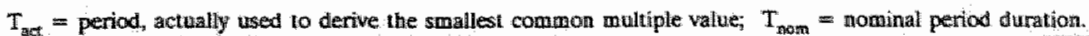

\begin{tabular}{|c|c|c|c|c|}
\hline frequency & 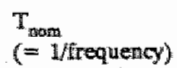 & $\mathrm{SCM} / \mathrm{T}_{\mathrm{nOm}}$ & 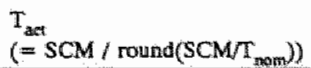 & $T_{\text {nom }}-T_{\text {axt }}$ \\
\hline 0.16 & 6.25000000 & $300.00000 \ldots$ & 6.2500000 & $0.0000000 \ldots$ \\
\hline 0.41 & 24390243 & $768.75000 \ldots$ & 2.4382314 & 0.0007929 \\
\hline 0.53 & 1.8867924 & $993.75000 \ldots$ & 1.8863179 & 0.0004745 \\
\hline 1.15 & 0.8695652 & 2156.25000 & $0.8696660 \mathrm{~m}$ & $0.0001008^{n}$ \\
\hline
\end{tabular}

the total period time.

In order to preclude prediction, it is important that the stimulus repeat period should exceed the measurement period. 


\section{Effect of asynchronous visual stimulation on the Vestibullo-Ocular Reflex coherence of normals.}

\subsection{Abstract.}

A vestibular function test (named Visuo-Vestibular Interaction Test: VVIT) is presented, in which a visual target may move asynchronously with a pseudo-randomly oscillating rotatory chair.

Here, normative data are given for the coherence, while the gain and the phase shift are considered elsewhere (chapters 5 and 6 , respectively).

\subsection{Introduction.}

A full introduction of the VVIT was given earlier (chapter 5), so here only the essentials will be repeated.

In order to elude the variation inherent to measuring the vestibulo-ocular reflex (VOR) in the dark, a visual target is presented. The usual ways in which the resulting visuo-vestibular interaction (VVI) is studied, (namely, by offering either an earth-fixed target or a chair-fixed target) allow, but may not require, the two systems to interact. To force an interaction between smonth pursuit (SP) and VOR, we use a visual target which moves asynchronously with the chair. Predictive effects are reduced by having the chair move in a pseudo random (sum-of-sines) way. As both visual target movement and head movement is below the SP upper limit, we expect normals and patients to perform equally well to either one of them. But because the combination is above the SP upper limit, we expect patients to perform worse than normals to the combined stimulus, assuming that they will overburden their SP system in an effort to compensate for the insufficient vestibular fucntion. By subtracting the visuo-vestibular interaction with an earth-fixed target (eftVVI; i.e., visually enhanced VOR) stimulus and the response to it, from the visuo-vestibular interaction with an asynchronously moving target (mtVVI) stimulus and the response to it, the visual components are obtained. It is hypothesized that the vestibular function is reflected by the ratio of the smooth and saccadic eye movements (and thus by the gain of the median-filtered response). Different coherences for normals and patients and for eftVVI and mtVVI might be expected in relation to the complexity of the stimulus and the type of neural processing it is subjected to. 


\subsection{Material and methods.}

\subsubsection{Subjects.}

Six controls ( 3 males of 23,33 , and 37 years, and 3 females of 23,26 , and 34 years) were recruited among ourselves and our students, resulting in a lower median age than that of the usual patient population for our clinic.

None of the subjects had any history of vestibular problems, and none of them had any difficulty with visually guided eye movements. All subjects, even those usely wearing glasses, could locate the visual target without any optical correction, and were therefore tested that way.

Three of them (two males of 23 and 37 years, and one female of 26 years) underwent the VVIT each at four different days in order to asses intra- and inter-subject variability.

\subsubsection{Experimental set-up.}

The experimental set-up was as reported previously (chapter 5), and so only a brief description follows here. Subjects sat in a rotary chair moving in a pseudo random way (sum-of-sines: $0.16,0.41$, and $1.15 \mathrm{~Hz}$, each with a maximum velocity of $10 \% / \mathrm{s}$ ), while watching a dot which was either earth-fixed or moving independently $(0.53 \mathrm{~Hz}$, with a maximum velocity of $32 \%$ ). The eye position was measured using infra red reflection, and the head velocity was monitored with an angular rate sensor. Eye position, head velocity, and visual target position were sampled at a $50 \mathrm{~Hz}$ frequency for 125 seconds by a 12 bit analog-to-digital $(A D)$ converter (the first two after being filtered analogously with a cut-off frequency of $10 \mathrm{~Hz}$ ) and were digitally stored for offline analysis.

\subsubsection{Digital signal analysis.}

Processing and analysis of the signals was as reported before (see chapter 5). In summary, position data were digitally differentiated to velocities, after which the total stimulus was combined from visual target movement and head movement. After median filtering and Welch windowing, half-overlapping segments of the data were Fourier transformed, and the power spectra derived from these, were averaged. Further frequency-averaging was done, until the product of segment-averaging and frequency-averaging equaled 40 (Bendat \& Piersol, 1971, p 191-194). The coherence 
function (Bendat \& Piersol, 1971, p337-339) was calculated, and finally those values at the response frequencies were extracted automatically from the spectrum using preset criteria. The individual contrasts of the eftVVI and the mtVVI measurements were calculated as relative differences, and were also analyzed statistically.

\subsubsection{Statistical analysis.}

The statistical analysis was as described earlier (chapter 5). In brief, responses were analyzed for stimulus frequency, subject, and the interaction between frequency and subject, by analysis of variance. The limits of the reference intervals were calculated in the standard way, so that a next, independent measurement of a normal subject has $95 \%$ chance to fall within this range. These ranges are equivalent to the ones advocated by Royston \& Matthews (1991) with error probabilities of only $0.55 \%$ and $0.83 \%$.

\subsection{Results and discussion.}

As might be expected for reponses of normals to stimuli chosen to lie within normal capabilities, the obtained coherence values are very close to unity. In figures 7-1 and

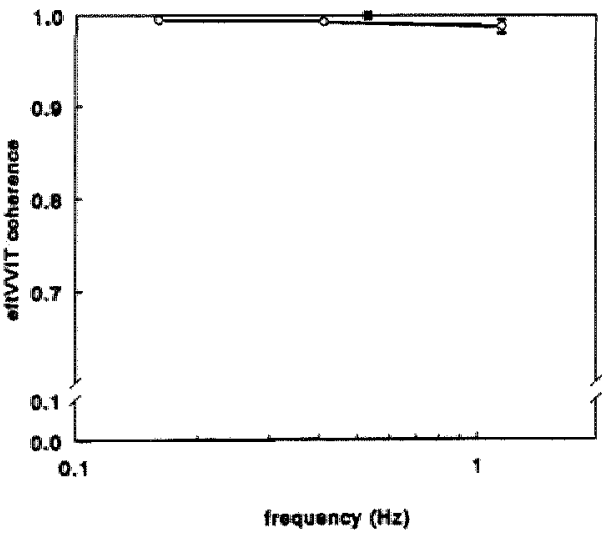

Fige 7-1. Coherence values for smooth pursuil ( $=$ = visual target) and eftVVI ( $O=$ chair). The single-sine smooth pursuit was conducted separatelly from the sum-ot-sines eftVVI stimulation.

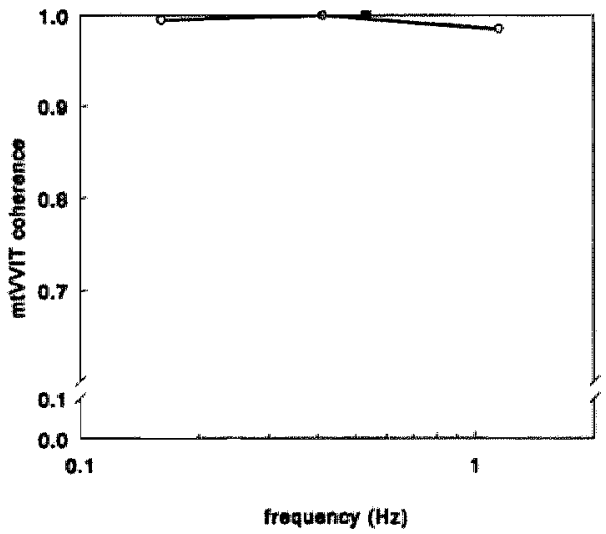

Fis $7-2$ Coherence values for visuowestibulary induced eye movements $(0=$ chair) and visually induced ones (visual target). All four frequencies were delivered simultanously by miVVI stimulation. 


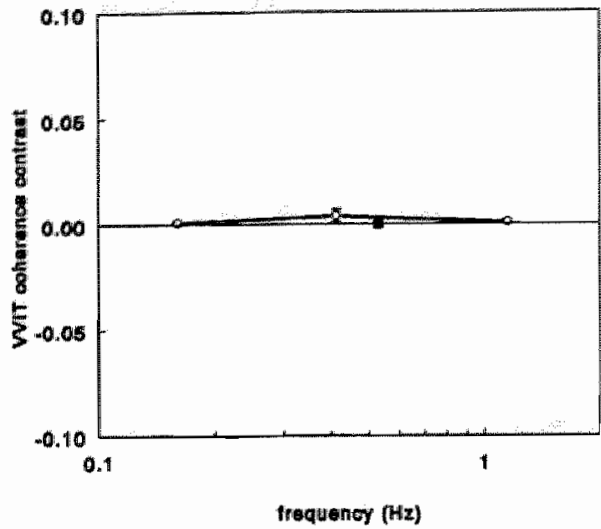

Fig. 7-3. Mean 4 standard error of the relative difference of coherence values resulting from mivVI and etr VVI stimula-

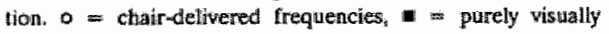
deliwered trequency.

7-2 they are given for eftVVI stimulation and for mtVVI stimulation, respectivelly. The increase of the coherence at $0.41 \mathrm{~Hz}$ when the visual target moves, might be an artefact. The visual target moved at a frequency of $0.53 \mathrm{~Hz}$, which is rather close to the middle chair-delivered frequency. Because frequency bins were averaged prior to calculating the coherence (in order to attain sufficient statistical indepencency) the higher value may result from the closiness of two peaks. Figures 7-3 and 7-4 show the

Table \%-1 Resulu of MANOVA on WVIT colterence waliues. Three subjects, three frequencies, four independent measurements per subject - frequency combiniation.

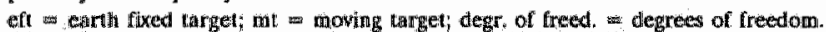

\begin{tabular}{|c|c|c|c|c|}
\hline \multirow{2}{*}{$\begin{array}{l}\text { tesit } \\
\text { atinatysilis }\end{array}$} & \multirow{2}{*}{$\begin{array}{l}\text { significat sourcefs) of } \\
\text { wariation (bestdes Intra- } \\
\text { individual wariability) }\end{array}$} & \multirow{2}{*}{ 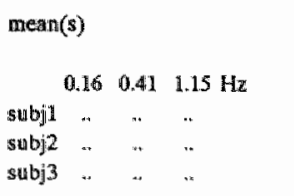 } & \multicolumn{2}{|c|}{ variance } \\
\hline & & & $\begin{array}{l}\text { intra: } \\
\text { individual } \\
\text { (degr. of freed) }\end{array}$ & $\begin{array}{l}\text { inter: } \\
\text { individual } \\
\text { (degr. of freed }=2 \text { ) }\end{array}$ \\
\hline eft & $\begin{array}{l}\text { inter-indiwidual wariation } \\
(\mathrm{p}<0.00 \mathrm{l})\end{array}$ & $\begin{array}{l}1.000 \\
1.0001 \\
0.993\end{array}$ & $\begin{array}{l}24 \times 10^{-6} \\
(31)\end{array}$ & $225 \times 10^{-6}$ \\
\hline $\begin{array}{l}\text { mt } \\
\text { coherence }\end{array}$ & $\begin{array}{l}\text { 1. stubject - frequency inter } \\
\text { action }(p<0.025) \\
\text { 2. frequency }(p<0.01)\end{array}$ & $\begin{array}{lll}0.995 & 1.000 & 0.995 \\
0.995 & 1.000 & 0.968 \\
0.993 & 1.000 & 0.970\end{array}$ & $\begin{array}{l}83 \times 10^{-6} \\
27)\end{array}$ & $336 \times 10^{6}$ \\
\hline $\begin{array}{l}\text { colterence } \\
\text { contrast }\end{array}$ & frequency $(p<0.001)$ & $2.5 \times 10^{3} \quad 2.3 \times 10^{3} \quad 9.4 \times 10^{3}$ & $\begin{array}{l}36 \times 10^{-15} \\
(31)\end{array}$ & $74 \times 10^{6}$ \\
\hline
\end{tabular}


Table 7-2 Lower and upper limits, defining normal WWIT responises. Bold printing guides choosing benween frequency-specific and frequency averaged limils, according to MANONA-estabilished significance of stiawilus frequency. Surpassing the parenthezed limats probably does not indicate a pathological condition.

\begin{tabular}{|c|c|c|c|c|c|c|}
\hline & \multicolumn{4}{|c|}{ chasir-delivered frequencies } & \multirow{2}{*}{$\frac{\text { visual }}{0.53 \mathrm{~Hz}}$} & \\
\hline & $0.16 \mathrm{~Hz}$ & $0.41 \mathrm{~Hz}$ & $1.15 \mathrm{Ftz}$ & $\begin{array}{l}\text { frequemcy- } \\
\text { averaged }\end{array}$ & & \\
\hline \multirow[t]{2}{*}{ muVIT coherence } & 0.980 & 0.995 & 0.945 & -0.975 & 1,000 & lower limit \\
\hline & 1.000 & 1.000 & 1.000 & 1.0000 & 1.000 & upper lìmit \\
\hline \multirow[t]{2}{*}{ VVIT coherence contrast } & 0.010 & 0.015 & -0.025 & -0.015 & 0.000 & lower limit \\
\hline & $(0.010)$ & $(0.0200)$ & $(0.020)$ & $(0.015)$ & 0.000 & upper limit \\
\hline
\end{tabular}

individually calculated relative differences between mtVVI and eftVVI responses. The first figure of this pair shows the mean \pm the standard error per chair-delivered frequency, and the second shows the individual values plotted in a perspectivic way for the three chair-delivered frequencies, using the limits of the univariate reference intervals as will be given below in table 7-2. The apparent little correlation of the coherence contrast for the three frequencies is largely due to the controls' lack of variation, as follows from comparing figure 7-4 with figure 10-12 from chapter 10 "Effect of asynchronous visual stimulation on the Vestibulo-Ocular Reflex coherence of patients, compared to that of normals".

Table 7-1 gives the results of analysis of variance with a repeated measurement design. It is found that during eftVVI stimulation, the subjects differed in their responses, while frequency did not influence the results. During mtVVI stimulation, however, frequency and the interaction between subject and frequency were important too. Taking the individual contrasts removed the inter-individual variation (or rather, increased the intra-individual variation so much, that the inter-individual variation is no longer significant at the $5 \%$ level), leaving the frequency-specific values.

Two reference ranges are calculated in such a way, that an independent, next measurement of a normal subject would have a chance of $95 \%$ to fall within those intervals. The resulting lower and upper limits are given in table 7-2. No range for eftVVI responses is given, because MANOVA of results from controls and patients failed to find significant differences related to group designation (see chapter 10 "Effect of asynchronous visual stimulation on the VOR coherence of patients, compared to that of normals"). Because in table 7-\# the subject-frequency interaction was found to influence results significantly in case of mtVVI stimulation, no frequen- 
cy-aweraging is allowed. The frequency-specific values are printed in bold to enphasize that these values should be refered to. Taking the individual differences made the inter-individual variation insignificant in table $7-1$ and so here the frequency: specific values should be used. Further, there is no obvious reason why the contrast values should not exceed some positive value (if subjects perform much better at the more difficult task than at the easier task, one could suspect a change in alertness; rather than some pathology), and so those upper limits are printed within paren. theses. For the frequency at which was stimulated only visually, all six subjects had a coherence of 1.000 , and so no reference range can be computed. 


\section{Effect of asynchronous visual stimulation on the Vestibulo-Ocular Reflex gain of patients, compared to that of normals. \\ 8.1 Abstract.}

A vestibular function test (named Visuo-Vestibular Interaction Test: VVIT) is presented, in which a visual target may move asynchronously with a rotatory chair. The individual differences in gain for two modes of stimulation ( 1 sum-of-sines chair movement with an earth-fixed visual target, $\underline{2}$ sum-of-sines chair movement with an independent sinusoidal target movement) are statistically analyzed for six groups of different types of vestibular patients (totalling 42 subjects) and a control group ( 6 subjects). Patients were diagnosed using anamneses and standard tests.

Patients having peripheral vestibular dysfunctions (well compensated, as well as poorly compensated) and whiplash victims are shown to differ significantly $(p<0.05)$ from the controls in their VVIT responses. Patients having initial stages of Meniere's syndrome and those having benign paroxysmal positioning dizziness (BPPD) are seen to differ suggestively, but not significantly $(0.05<\mathrm{p}<0.10)$ from the controls. Patients for whom no causes for their vestibular complaints were known, did differ neither significantly nor suggestively $(p=0.118)$ from the controls.

Using a reference range based on controls only, sensitivities of $1.00,0.63,0.54,0.70$, 1.00 , and 0.50 were obtained for Meniere patients, for well compensated and for poorly compensated peripheral vestibular patients, for whiplash victims, for BPPD patients, and for those for whom no cause is known, respectively. With respect to controls only, specificity was 1.00 for all six diagnostic groups. With respect to all other vestibular patients and controls, and for a single stimulus frequency, the best combination of sensitivity and specificity ( 1.00 and 0.70 , respectively) was found for Meniere patients.

\subsection{Introduction.}

A full introduction of the VVIT was given earlier (chapter 5: "Effect of asynchronous visual stimulation on the Vestibulo-Ocular Reflex gain of normals"), so here only the essentials will be repeated.

In order to elude the variation inherent to measuring the vestibulo-ocular reflex (VOR) in the dark, a visual target is presented. The usual ways in which the resulting visuo-vestibular interaction (VVI) is studied, (namely, by offering either an earth-fixed target or a chair-fixed target) allow, but may not require, the two systems to interact. To force an interaction between smooth pursuit (SP) and VOR, we use a visual target which moves asynchronously with the chair. Predictive effects are reduced by having 
the chair move in a pseudo random (sum-of-sines) way. As both visual target movement and chair movement are below the SP upper limit, we expect normals and patients to perform equally well to either one of them. But because the combination is above the SP upper limit, we expect patients to perform worse than normals to the combined stimulus, assuming that they will overburden their SP system in an effort to compensate for the insufficient vestibular function. By subtracting the visuo-vestibulaf interaction with an earth-fixed target (eftVVI; i.e., visually enhanced VOR) stimulus and the response to it, from the visuo-vestibular interaction with an asynchronously moving target (mtVVI) stimulus and the response to it, the visual components are obtained. It is hypothesized that the vestibular function is reflected by the ratio of the smooth and saccadic eye movements (and thus by the gain of the median-filtered response).

\subsection{Material and methods. 8.3.1 Subjects.}

Every patient entering our vestibular department during the period of 920518 .. 920914 was included in this study, unless anamnesis strongly suggested other causes of vestibular complaints than horizontal semicircular canal functioning and related central processing. Of the 64 patients examined with the Visuo-Vestibular Interaction Test (VVIT; to be described below) as well as with standard tests, 13 were excluded from analysis because stimulation was found to have been differing from the intended one. Fifty-one patients remained for analysis. Because during the study whiplash victims appeared to respond very differently from the others, more of them were referred to us by dr. J. Patijn from the Foundation for Manual Therapy in Eindhoven, and so this group is overrepresented. An experienced vestibulologist (dr. $\mathrm{H}$. Kingma) routinely diagnosed the patients according to their anamneses and their performance at standard tests prior to being informed about their VVTT outcomes. Six diagnostic groups having three or more members emerged: those having initial stages of Meniere's syndrome (4), those having peripheral vestibular dysfunctions, centrally well compensated (8) and centrally partly compensated (13), whiplash victims (10), BPPD patients (3), and those for which the causes of their complaints are unknown (4), making a total of 42 patients. The nine other patients were distributed over six diagnostic groups, and these data were not analyzed. No other selections were made.

Six controls were recruited among ourselves and our students, resulting in a smaller age range (23.37) and lower median age (30) than that of the patients (13..81, and 45 , respectively). 
None of the subjects had problems with visually gujded eye movements. All subjects, even those usually wearing glasses, could locate the visual target without any optical correction, and were therefore tested that way.

\subsubsection{Experimental set-up.}

The experimental set-up was as reported previously (chapter 5), and so only a brief description follows here. Subjects sat in a rotary chair moving in a pseudo random way (sum-of-sines: $0.16,0.41$, and $1.15 \mathrm{~Hz}$, each with a maximum velocity of $10 \%$ ), while watching a dot which was either earth-fixed or moving independently $(0.53 \mathrm{~Hz}$, with a maximum velocity of $32 \% \mathrm{~s}$ ). The eye position was measured using infra red reflection, and the head velocity was monitored with an angular rate sensor. Eye position, head velocity, and visual target position were sampled at a $50 \mathrm{~Hz}$ frequency for 125 seconds by a 12 bit analog-to-digital $(\mathrm{AD})$ converter (the first two after being filtered analogously with a cut-off frequency of $10 \mathrm{~Hz}$ ) and were digitally stored for offline analysis.

Standard tests consisted of calorization $\left(30^{\circ}\right.$ Celsius and $44^{\circ}$ Celsius, in the order right cold, left cold, left warm, right warm), torsion in the dark $(0.1 \mathrm{~Hz}$ frequency, 50 $\% / \mathrm{s}$ peak velocity), a smooth pursuit test $\left(0.29 \mathrm{~Hz}\right.$ frequency, $15^{\circ}$ amplitude), and an optokinetic test $(10,20,30$, and $40 \% / \mathrm{s}$ velocity, both horair and anti-horair). Left and right horizontal eye position was measured by electro-oculography, and was sampled at $100 \mathrm{~Hz}$.

\subsubsection{Digital signal analysis.}

Processing and analysis of the signals was as reported before (see chapter 5). In summary, position data were digitally differentiated to velocities, after which the total stimulus was combined from visual target movement and chair movement. After median filtering and Welch windowing, half-overlapping segments of the data were Fourier transformed, and the power spectra derived from these, were averaged. The gain spectrum was calculated from these power spectra, and finally the gain values at the response frequencies were extracted automatically from the spectrum using preset criteria. The individual contrasts of the eftVVI and the mtVVI measurements were calculated as relative differences, and were also analyzed statistically. 


\subsubsection{Statistical analysis.}

Statistical analysis was done using SPSS software (SPSS/PC+, Chicago, USA) for multivariate analysis of variance (MANOVA). Univariate Normal distribution per group-frequency combination was checked by inspecting detrended cumulative Normal probability plots. In case of non-Normality, first the parameter for the Box-Cox transformation (Sokal \& Rohlf, 1981, p 423-427) was calculated, and if this did not suggest scaling-dependent deviations, non-Normal outliers were excluded (set appendix). MANOVA was used to assess the significances with which various factors correlated with the observed differences of the means. Four influences were analyzed: the within-subject factor stimulus frequency, the between-subjects factors sex and diagnosis, and the covariate age.

Sensitivity was calculated per diagnostic group for two reference ranges (a standard one (Albert \& Harris, 1987) and an unbiased one (Royston \& Matthews, 1991); see chapter 5) in three ways ( 1 using each subject's best single result, 2 lumping together intra-subject and inter-subject results; $\underline{3}$ labelling subjects as patients if any of their intra-subject results was outside the reference ranges). Specificity was calculated regarding controls only, as well as regarding other diagnostic groups and controls.

\subsection{Results and discussion.}

Because gain contrast is not influenced by calibrational errors, only these values have been analyzed. The Visuo-Vestibular Interaction using a sum-of-sines chair movement and a asynchronously (single-sine) moving visual target (mtVVI) is compared with the VVI using an earth-fixed visual target (eftVVI) (eft- and mtVVI gain values are given in figure 8-1). In figures 8-2 and 8-3 the VVIT gain contrasts (mean \pm standard error) are depicted per group and per chair-delivered stimulus frequency. Conform the statistical analysis to be described below, the patient groups are divided in those whe differ significantly from the controls and those who do not, and outlying values are excluded from the means and standard errors. And figure 8-4 shows the gain contrasts for the purely visually delivered stimulus frequency.

The mean gain contrast of the controls is positive, but is so small that it does not differ significantly from zero (see chapter 5 ). In contrast, all studied patients groups have on average negative values, indicating that they performed worse at the mtVVIT than at the eftVVIT. As described in the introduction, this is as the test was intended to work.

Normal distribution of the responses of the diagnostic groups containing three or 

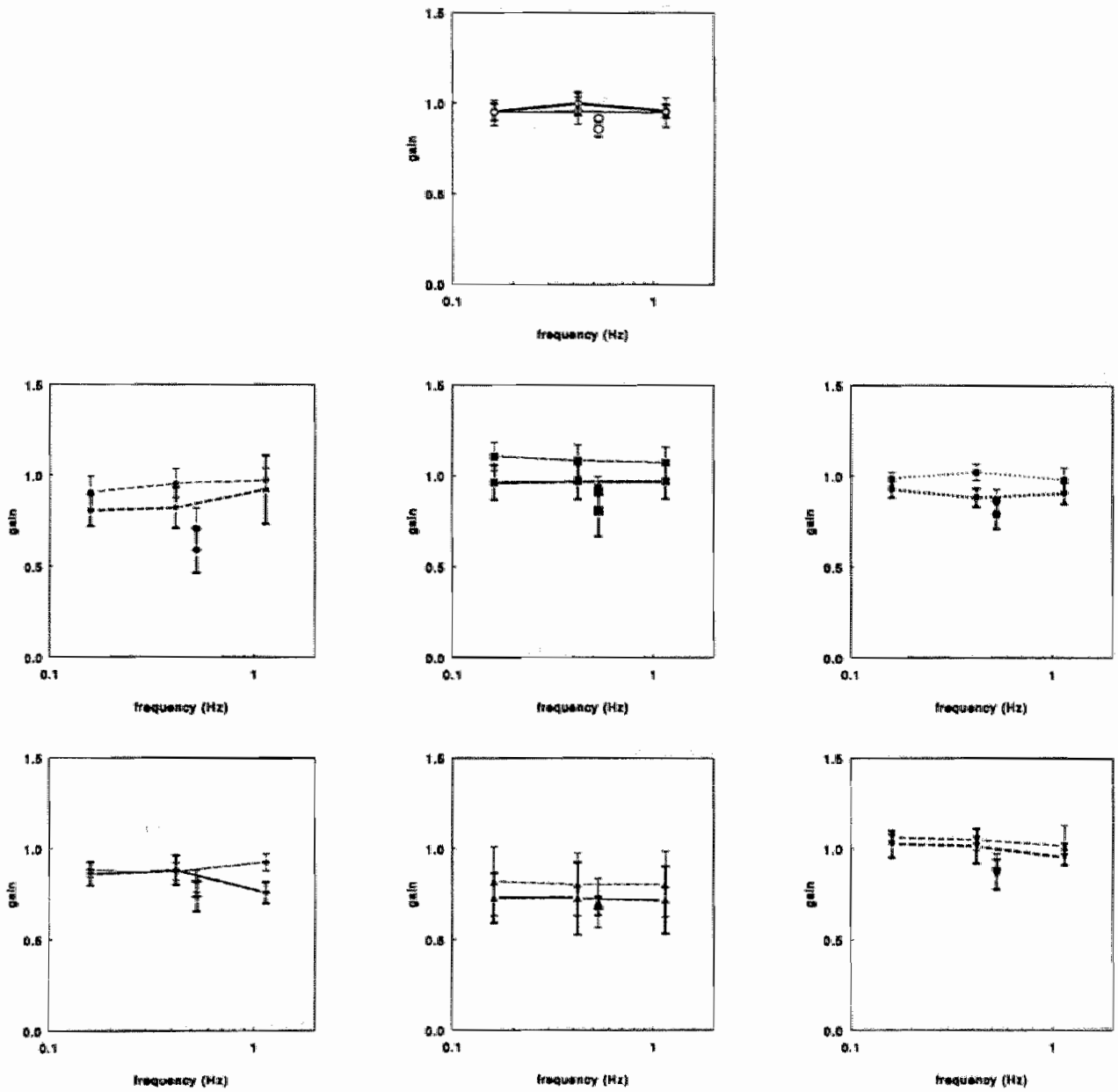

Fig. S-1. Group averages for gain values per stimulus frequency (small symbols: $0.16,0.41,1.15$ Hz frequencies delivered by the rotatory chair, large symbols: $0.53 \mathrm{~Hz}$; (requency delivered by visual target actual movement): 0 controls, patienis having initial stages of Menilere's disease, $=$ = patients having peripheral vestibtilar dysfuncions "which are centrally well compensated $\bullet$ - patienis having peripheral vestibular dysfunctions, which are centrally poorly conpensated, + patients having whiplash trama, $4=$ patients having benign paroxysmal positioning dizziness, $=$ patients having wertigo for which the origin is unknown. The average values for the chair-delivered frectuencies (small symbols) are either connected by thin lines indicating averages obtained with the earth-fixed target VVI paradign, or fat lines indicating those obtained with the thowing target VVI paradign. At the frequency of the visual target movement ( $0.53 \mathrm{~Hz}$; large symbols), the higher gain values stem from the smooth pursuit shimulas, and the lower ones from miVI.

Differences in gain between groups as well as between purely visually delivered and chair-delivered frequencies may neflect differences in calibyration. 


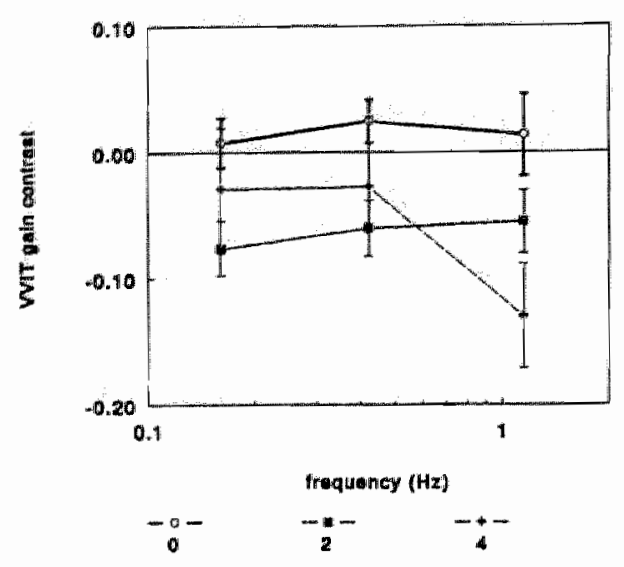

Fug 8-2 VWT gain conirast depending on stimulus Lrequency for convrols ( $(0)$ and signiticantly different patienis: (- weil compensated, peripheral westibular dystunction, + swiplasth trama).

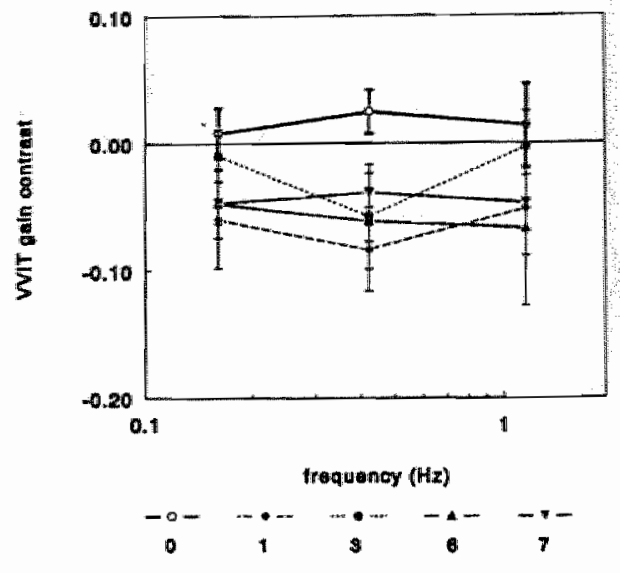

Fig. 8-3. VVIT gain contrast depending on stimulus frequency for controls $(0)$ and not significantly different patients ( peripheral vestibular dysifunction, * = benign panoxysmall positioning vertigo, $\nabla=$ unknown).

more members, was checked by means of Normal plots (results not shown; see appendix). Deviations were found and then two strategies are possible for Normalizing the data. If one considers all data equally important, then transforming the data can be tried. Alternatively, some data can be considered outliers and can be excluded.
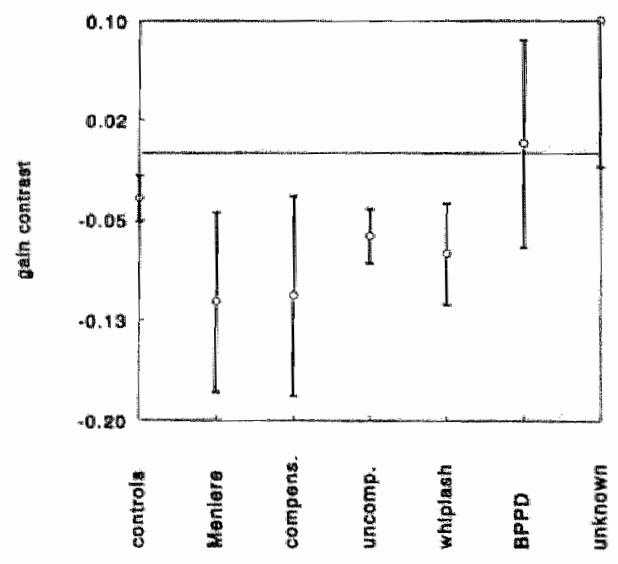

Fig- 8.4. Gain contrast relative difrerences between singlesine $(0.53 \mathrm{H} x)$ vistual target movement, simultanous and asymchronous with the sumof-sines chair mowement, and idem without chair wovement. Same vertical scaling as Ingures $8-2$ and $8-3$.
Values for the parameter $\lambda$ for corrective Box-Cox transformations (see appendix) were calculated for each frequency - group combination (table 8-1).

It can be seen that values vary considerably, suggesting that the observed deviations from Normality are not caused by inappropriate scaling. Singling out the combinations with the most severe deviations (of 0.5 units or more; indicated by bold printing in table 8-1) still gave various values for $\lambda$. The median value $(\lambda=3.7)$ was used for a Box-Cox transformation of all frequency - group combinations. Evaluating the transformed data by means of detrended Normal plots showed no overall improvement (data not shown). Not sur- 
Table 8-1. Walues for the parameter $\lambda$ of the Box-Ciox transformation for various frexuincy-group combinations. Bold printed nalue indicate combinations that deviate 0.5 units or mone from the Normal distribution.

\begin{tabular}{|c|c|c|c|}
\hline diagnasis & $\begin{array}{l}0.16 \\
\mathrm{~Hz}\end{array}$ & $\begin{array}{l}M 411 \\
H z\end{array}$ & $\begin{array}{l}1.15 \\
\mathbb{H z}\end{array}$ \\
\hline 0. controls & -2.5 & 3.3 & -4.9 \\
\hline In initial stages of Meniere's syndrome & -7.1 & -42 & 0.0 \\
\hline 2 peripheral vestibular dysfunction, centrally well compensated & 4.2 & 9.4 & 81 \\
\hline 3. peripheral vestibular dysfunction, centrally poorly compensated & 6.1 & 4.6 & 3.4 \\
\hline 4. Whiplasit trauma & 39 & 24 & $1 \pi 2$ \\
\hline 6. beaign parbxysmal positionuing diaziness & 115 & -0.5 & 6.0 \\
\hline 7. Westibular complainus from unknown causes & 7.8 & $\$ .5$ & 7.4 \\
\hline
\end{tabular}

prisingly, Normality of the combinations for which $\lambda$ was near 3.7 , had improved while it had detoriated for the other combinations.

Using the same criterion of deviating 0.5 units or more from the Normal distribution, with an extra requirement of lying distinct from the bulk of the data, resulted in detecting six outliers (table 8-2). These were excluded together with the results for the other frequencies of those subjects. The remaining data approximated the Normal distribution better, and so subsequent analysis was done on this set.

The overall testing (table 8-3) estimated $(-5.5 \pm 2.5) \times 10^{-2} \times$ age to be the effect of age on the gain contrast, which is significant $(p=0.032)$. Sex did also influence results significantly $(\mathrm{p}=0.002$ ), depending on stimulus frequency and diagnostic group. This latter makes it a bit suspicious, because it seems improbable that some vestibular dysfunctions will be sex-specific, while other are not.

Table 8-2 Outlying response values for various frequency - growp comabinations. Criterion was deviatimg 0.5 untis or mote from the Normal distribution as well as lyng distinct from the bulk of data.

\begin{tabular}{|c|c|c|c|}
\hline diagnosis & $\begin{array}{l}0.16 \\
H_{z}\end{array}$ & $\begin{array}{l}0.41 \\
\mathrm{~Hz}\end{array}$ & $\begin{array}{l}1.15 \\
\mathrm{H} z\end{array}$ \\
\hline 0. contirculs & $\ldots$ & .. & . \\
\hline 1. inhütal stages of Meniere"s syndrome & -. & .. & $\therefore$ \\
\hline 2. peripheral westibular dysfunction, centrally well compensated & $\cdots$ & $*$ & $\because$ \\
\hline 3. peripheral westibular dysfunction, centrally poorly compensated & .. & 0.295 & -0.218 \\
\hline 4. whiplast trauma & .0 .394 & 0.396 & $n$ \\
\hline 6. benign paroxysmall positioning dizzinexts & $m$ & $\cdots$ & $\therefore$ \\
\hline 7. vestibular complaints from unknown causes & . & -0.400 & - \\
\hline
\end{tabular}




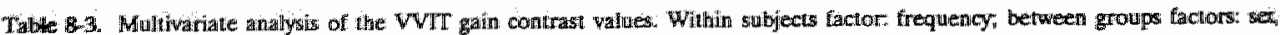
diagnosis; coratiate: age Stans indicatg impontan group differences.

\begin{tabular}{|c|c|c|c|c|c|c|c|}
\hline \multicolumn{8}{|c|}{$\begin{array}{l}\text { sidnificance of independent variables } \\
\qquad(\mathrm{p}=\end{array}$} \\
\hline age & $\begin{array}{l}\text { distgin (afler } \\
\text { interactiten) }\end{array}$ & $\begin{array}{l}\text { sest (after } \\
\text { interaction) }\end{array}$ & $\begin{array}{l}\text { Wheraction of } \\
\text { sex, diag and } \\
\text { freg. }\end{array}$ & $\begin{array}{l}\text { interaction of } \\
\text { sex aind diag. }\end{array}$ & $\begin{array}{l}\text { Interaction } \\
\text { of sex and } \\
\text { freq. }\end{array}$ & $\begin{array}{l}\text { interaction } \\
\text { of diagn. } \\
\text { and frec. }\end{array}$ & $\begin{array}{l}\text { effect of } \\
\text { freq- (affer } \\
\text { intergetions) }\end{array}$ \\
\hline 0.032 & $\begin{array}{r}0.005 \\
+4\end{array}$ & 0.525 & $\begin{array}{r}0.002 \\
\times \times\end{array}$ & 0.160 & 0.945 & $\begin{array}{r}0.007 \\
\times \times \\
\end{array}$ & 0.944 \\
\hline
\end{tabular}

+ significanit at I\% level

Because overall testing showed another significant difference between groups (for the interaction of diagnosis and stimulus frequency; $p=0.007$ ), each patient group separately may be compared with the controls.

The results of pairwise testing are shown in tables 8-4 and 8-5.

Age is not important in these pairwise comparisons, and so it should be excluded from analysis. Sex is a significant factor for group 6 only, so other groups should be compared without sex as an explaining variable. The results of the reduced MANO-

Table 8-4. MANOVA results of painwise comparison of VVIT gain contrast walues from warious patient groups with a control group $(n=6)$, whilh seat, diagnosis and stimulus trequency as independent variables and age as covariate. Stars indicate ümportant group diffierences:

\begin{tabular}{|c|c|c|c|c|c|c|c|}
\hline \multirow[t]{2}{*}{ diagnosis } & \multirow{2}{*}{$\begin{array}{l}\text { number } \\
\text { of mem- } \\
\text { fuers }\end{array}$} & \multicolumn{6}{|c|}{$\begin{array}{c}\text { significance } \\
(p=. .)\end{array}$} \\
\hline & & age & $\begin{array}{l}\text { diagnosis } \\
\text { (after } \\
\text { interac- } \\
\text { thon) }\end{array}$ & $\begin{array}{l}\text { sex (after } \\
\text { interac- } \\
\text { tion) }\end{array}$ & $\begin{array}{l}\text { interac- } \\
\text { tion of } \\
\text { sex and } \\
\text { trequency }\end{array}$ & $\begin{array}{l}\text { interac- } \\
\text { tion of } \\
\text { diagnosis } \\
\text { and fre- } \\
\text { quenny }\end{array}$ & $\begin{array}{l}\text { frequency } \\
\text { (after } \\
\text { interac- } \\
\text { tion) }\end{array}$ \\
\hline $\begin{array}{l}\text { 1. finit lial stages of Meniere's } \\
\text { 5ymdirome }\end{array}$ & 4 & $0: 390$ & 0.187 & 0.700 & 0.835 & 0.587 & 0.891 \\
\hline $\begin{array}{l}\text { 2. peripheral veribular dys- } \\
\text { funcilon, well compensated }\end{array}$ & 8 & 0.540 & 0.279 & 0.281 & 0,958 & 0.837 & 0.472 \\
\hline $\begin{array}{l}\text { 3. peripheral westibutar dys- } \\
\text { furiction, poorly compensaled. }\end{array}$ & $\Perp 1$ & 0.242 & 0.277 & 0.668 & 0.774 & 0.204 & 0.548 \\
\hline 4. Whiplash trauma & 9 & 0.734 & 0.527 & 0.925 & 0.703 & $0.076(t)$ & 0.244 \\
\hline $\begin{array}{l}\text { 6. benign paroxysma } \\
\text { pasitionahg diaziness }\end{array}$ & 3 & 0.766 & 0.121 & 0.732 & 9.005 t & 0.109 & 0.577 \\
\hline 7. unkriown causes & 3 & 0.738 & 0.251 & 0.505 & 0.589 & 0.589 & 0.055 \\
\hline
\end{tabular}


Table 8-5. MANOVA resutus of paintise comparison of WhT gain contrast wallses from warious patient groups with a control group $(n=6)$, whith diagrosis and stimudus frequency as independent variables. Stars indicate importamt govop differences.

\begin{tabular}{|c|c|c|c|c|}
\hline \multirow[t]{2}{*}{ diagnosis } & \multirow{2}{*}{$\begin{array}{l}\text { number } \\
\text { of :mem- } \\
\text { bers }\end{array}$} & \multicolumn{3}{|c|}{$\begin{array}{l}\text { Significance } \\
(\mathrm{P}=0)\end{array}$} \\
\hline & & $\begin{array}{l}\text { ditignosis } \\
\text { (after } \\
\text { interac: } \\
\text { tion) }\end{array}$ & $\begin{array}{l}\text { interas- } \\
\text { thon of } \\
\text { diagnosis } \\
\text { and fre- } \\
\text { quency }\end{array}$ & $\begin{array}{l}\text { frequency } \\
\text { (after } \\
\text { interac- } \\
\text { tion) }\end{array}$ \\
\hline 1. invitial stages of Meniere's syndrome & 4 & $0.099(-)$ & 0.625 & 0.908 \\
\hline 2. peripheral vesubular dysfunction, well compensated & $\$$ & $0.021+r$ & 0.008 & 0.444 \\
\hline 3. peripheral vestibular dysfunction, poorly compensated. & 11 & 0.104 & 0.179 & 0.535 \\
\hline 4. whiplasts trauma & 9 & 0.263 & $0.053 \times$ & 0.193 \\
\hline 6. benign paroxysmal positioning dixziness ( ) & 3 & $0.064(+)$ & 0.696 & 0.899 \\
\hline 7. unknown causes & 3 & 0.118 & 0.440 & 0.028 \\
\hline
\end{tabular}

(*) suggestive, but not significant at $5 \%$ level
$\star$
(*) companticant at $5 \%$ level

VA are given in table 8-5. The finding in table 8-4 that BPPD patients (and only they) have a sex specific response, is rather unexpected. Perhaps it can be explained as a freak outcome due to its small sample size.

From table $8-5$ can be seen that for group $\mathbb{1}$ there is insufficient evidence to conclude already that these patients scored differently from the controls. But neither may one conclude that both groups are identical, given the small sample size and the rather low chance probability $(\mathrm{p}=0.099)$.

Compared to the controls, group 2 differs significantly $(p=0.021)$ in its overall response but not in its frequency-specific response, while group 6 reacts in a significantly $(\mathrm{p}=0.053)$ different way to the stimulus frequencies, but does not differ in overall response. Groups 3 and 7 do not distinct themselves from the controls. If one rejects the significance of sex influences on the VVIT gain contrast results for group 6 as a freak outcome, then the differences are attributed to diagnostic group designation, making it suggestive, but not yet significant at a $5 \%$ level.

When comparing the statistical test results with the graphical representations given in figures 8-1 and 8-2, two things have to be kept in mind. First, testing was done in a multivariate way, taking into account that each subject responded to various frequencies, making this data related. And secondly, the groups differed in size. These information is lost or is less obvious in the graphs. In general, graphs and statistical results agree. Figure 8-1 shows that group 4 differs in its frequency response from the controls (because the response at $1.15 \mathrm{~Hz}$ deviates from the others), and the statis- 

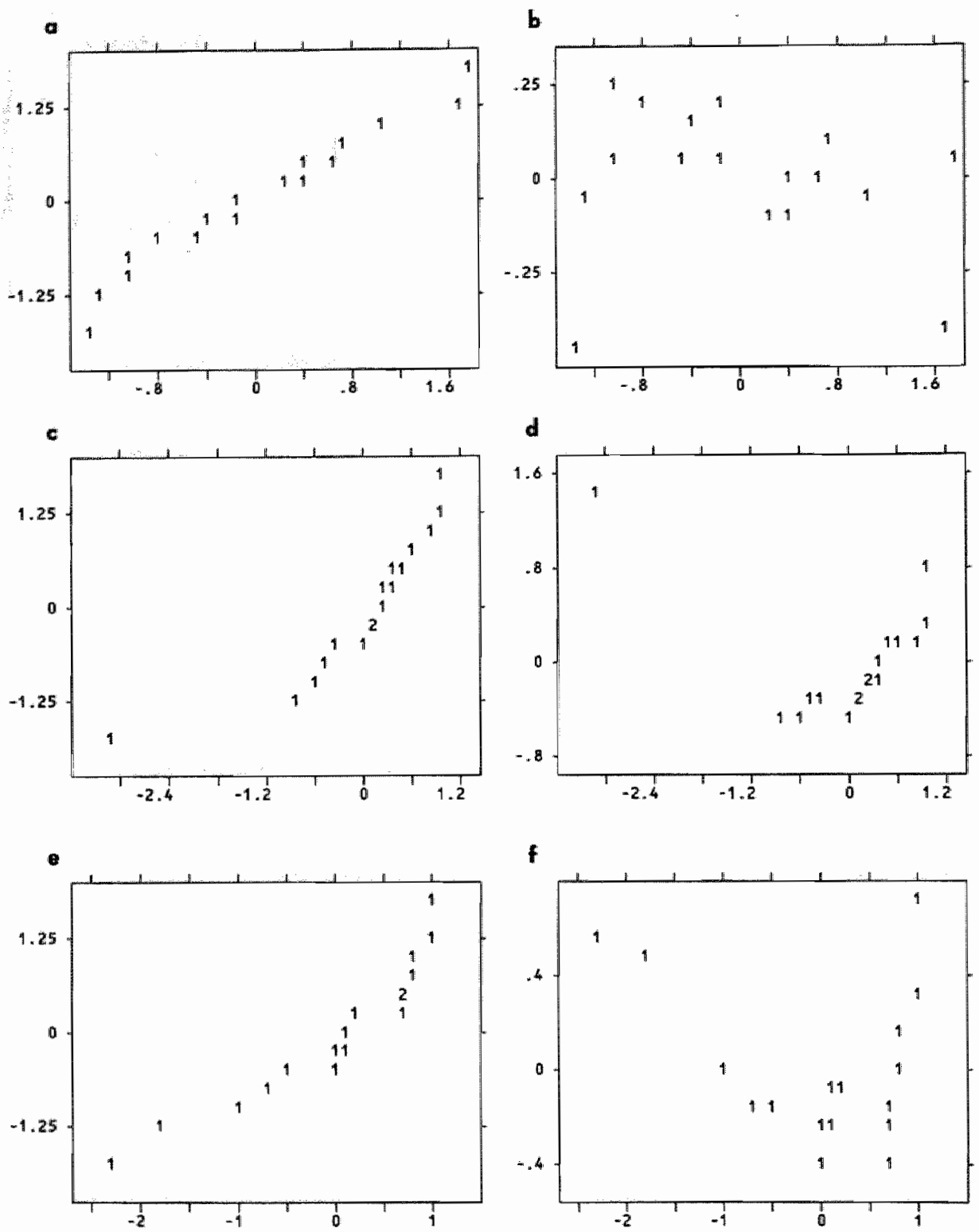

Fugre 8.5. Evaluation of the residuals from the MANOVA of the group of centrally partly adaphed peripheral westibularty dysfunctioning patients (group 3 ) and the controls.

a. Nomal probability plot for the constant. b. detrended Normal probability plot for the constant. c. Normal probability pirot for the second chair frequency $(f=0.41 \mathrm{~Hz}$ ). d detrented Normal probability plot for the second chair frequency. e. Normal probability plot for the third chair frequency $(\mathrm{f}-1.15 \mathrm{~Hz}$ ). L detrended Normal probability plot for the third chair frequency. 
tical test shows that this is the only difference for this groups that is significant. Further, it shows that group 2 differs in its overall response, but not in its frequency response, which is also confirmed by the statistical test. In figure 8-3 groups 1 and 6 differ in about the same way from the controls as group 2 does in figure 8-2. But probably because of the smaller group sizes ( 4 and 3 members, respectively, while group 2 has 8 members), it is not significant at the $5 \%$ level. However, they (and only they) are so at the $10 \%$ level. Group 3 is almost significantly different at the $10 \%$ level from the controls with re-

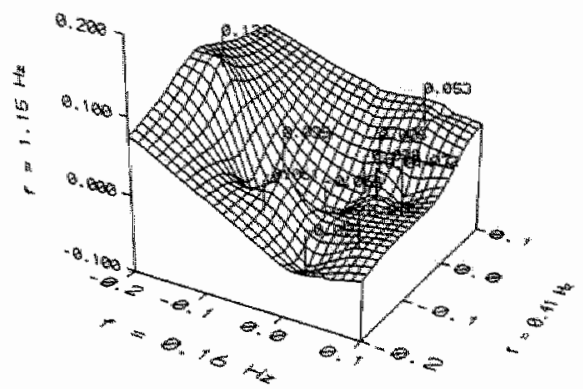

Fig. 8-6. Responses to the three chair frequencies for the group of partly compensated patients. Although each univariate distribution is approximately Normal, the mulltivariate distribution has one extreme oultier. spect to overall response, but not with respect to the frequency-specific responses. This seems to be contradicted by figure $8-3$, which shows a markedly different response at $0.42 \mathrm{~Hz}$. Such a discrepancy might be caused by the loss of multivariate information by graphical representation, but it might also result from the distribution being multivariately non-Normal (only the univariate distributions were checked and made Normal if necessary). Therefore, the Normality of each variable's residuals' distribution was checked for all analyzed groups. Only for group 3 did the residuals" plot suggest multivariate outliers (figure 8-5). To identify those outliers, the data for group 3 has been plotted in a perspective way in figure 8-6. And indeed, one triplet (i.c., $\{-0.159,-0.066,0.131\}$ ) is lying completely beside the bulk of the data. Repeating the MANOVA for the partly compensated peripheral vestibularly dysfunctioning patients versus the controls, with the multivariate outlier removed, produced the results shown in table 8-6. MANOVA

Table 86. MANOVA results of pairwise comparison of VWT gin contrast values from centrally partly compensated peripheral yestibular patients $(n=10)$ with a control group $(n=6)$, with diagnosis and stimulus frequency as indepuendut variabiles. One multivariate outlier was additionally remowed from the patiems* population, and therefore these resulus teplace those ginen in table 8-5. Stars indicate important group differenes.

\begin{tabular}{|c|c|c|c|}
\hline & \multicolumn{3}{|c|}{$\begin{array}{l}\text { significance } \\
(\mathrm{p}=\ldots .)\end{array}$} \\
\hline & $\begin{array}{l}\text { diagnosis (after } \\
\text { interaction) }\end{array}$ & $\begin{array}{l}\text { interaction of } \\
\text { diagnosis and } \\
\text { frequency }\end{array}$ & $\begin{array}{l}\text { frequency (after } \\
\text { interaction) }\end{array}$ \\
\hline full MANOVA & \multirow[t]{2}{*}{0.129} & 0.101 & 0.437 \\
\hline MANONA, with repeated measures averaged & & $0.038 \star$ & 0.321 \\
\hline
\end{tabular}


found no significant effect of the interaction of diagnosis and stimulus frequency ( $\mathrm{p}=$ 0.101). But because also no correlation was found between the responses within each subject (significance according to the Mauchly sphericity test: $p=0.544$ ), the averaged results of the repeated measures may be used, and because the corrective factors are close to 1 (Greenhouse-Geiser: 0.91802, Huynh-Feldt: 1.00000), the degrees of freedom ( 2 and 28 ) are little effected. So the probability that the observed differences are completely due to chance, is a little higher than the indicated 0.038 , but it remains below 0.05 .

To assess the clinical usefulness of a test, its sensitivity (i.e., the ratio of found and actual patients) and its specificity (i.e., the ratio of found and actual non-patients) are calculated (e.g., Sacket et al., 1985, p 69-72). This is straightforward in case of a univariate test and one type of patients, but becomes a little more complicated in our case. The current VVTT produces 26 results", and in the current study the results of six types of patients ${ }^{2}$ are analyzed. Of course, all the data generated by the VVIT should be used to determine whether a subject is a patient, and if so, what type of patient he is. This can be done by combining the results with weights as found by discriminant analysis (see chapter 11 "Discriminating power of VVIT for type of pathology, compared to those of standard tests"). With these end results the sensitivity and specificity can be calculated in the usual way. Nevertheless, it is also interesting to see whether the univariate results alone would allow us to draw valid conclusions, as that would greatly simplify diagnosis. Having various results, one may give the best sensitivity for a single variable-frequency combination (which can be different combinations for various groups of patients), the average sensitivity (Le., all positive test outcomes, divided by the product of the number of outcomes per test times the number of patients), or the "inclusive-or" sensitivity (i.e., the sensitivity when a subject is considered to be a patient if any of the test results deviates significantly from those of the normals). And one may give the specificity regarding controls only (i.e., no patients for any type of vestibular dysfunction), or for non-patients with respect to a specific type of vestibular dysfunction (and thus including other vestibular patients as well as controls).

Here, we restrict ourselves to gain contrast, while the results using the complete set of variable-frequency combinations are given for comparison. Table 8-7 shows that using the unbiased reference ranges and the "inclusive-or" criterium gives the best sensitivities, but also that gain contrast is not the most sensitive variable for four of

\footnotetext{
126 VVIT resulus" eftVII phase shiff and eftVVI coherence, both for three frequencies, and gain contrash, mutWl phase shift, phase shift difference, mivVI coherence, and coherence contrast, each for four frequencies.

2 Six types of patients: initial stages of Meniere's disease, centrally well compensated peripheral vestibular dysfunction, cemirallyy poorly compensated peripheral vestibular dysfunction, whiplash trauma, benign paroxysmal positioning dizziness, vestibular complaints from unknown origin.
} 
Table 8-7. Sensitivities of the VVTT for gain contrast only (four frequencies) and owerall (all varisible (requency combintions).

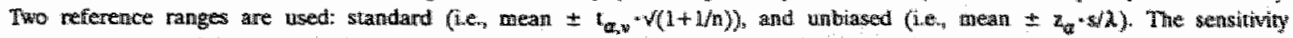
has been computed in three ways: the best single result per subject was used (best), no distinction wast made between test results per subject and test results from various subjects ("averaged"), and subjects were labeled patient, if any test result was deviant ("inclusive-or"). Specificity with respeet to controls is 1.00 for all three methods and for both ranges.

\begin{tabular}{|c|c|c|c|c|c|c|c|c|c|c|c|c|}
\hline \multirow[t]{2}{*}{ diagnosis. } & \multicolumn{6}{|c|}{ gain contrast sensitivity } & \multicolumn{6}{|c|}{ oxerall sensitivity } \\
\hline & best & $\begin{array}{l}\text { aver- } \\
\text { aged }\end{array}$ & $\begin{array}{l}\text { incL } \\
\text { or }\end{array}$ & best & $\begin{array}{l}\text { aver- } \\
\text { aged }\end{array}$ & inel. & best & $\begin{array}{l}\text { aver- } \\
\text { aged }\end{array}$ & $\begin{array}{l}\text { incl. } \\
\text { or }\end{array}$ & best & aver- & $\begin{array}{l}\text { incl. } \\
\text { or }\end{array}$ \\
\hline Menilere & 0.50 & 0.25 & 0.75 & 1.00 & 0.38 & 1.00 & 1.00 & 0.51 & 1.00 & 1.00 & 0.66 & 1.00 \\
\hline compensated & 0.25 & 0.16 & 0.50 & 0.38 & 0.31 & 0.63 & 0.75 & 0.34 & 0.88 & 0.75 & 0.42 & 0.88 \\
\hline uncompensated & 0.31 & 0.17 & 0.46 & 0.54 & 0.31 & 0.54 & 0.92 & 0.35 & 1.00 & 0.92 & 0.42 & 1.00 \\
\hline whipliash & 0.40 & 0.30 & 0.70 & 0.60 & 0.53 & 0.70 & 1.00 & 0.51 & 1.00 & 1.00 & 0.60 & 1.00 \\
\hline BPPD & 0.33 & 0.17 & 0.67 & 0.67 & 0.33 & 1.00 & 1.00 & 0.42 & 1.00 & 1.00 & 0.52 & 1.00 \\
\hline \multirow[t]{2}{*}{ Inknown } & 0.25 & 0.25 & 0.25 & 0.50 & 0.31 & 0.50 & 0.50 & 0.30 & 0.50 & 0.75 & 0.36 & 1.00 \\
\hline & \multicolumn{3}{|c|}{$\begin{array}{l}\text { standard } \\
\text { reference range }\end{array}$} & \multicolumn{3}{|c|}{$\begin{array}{l}\text { unbiased } \\
\text { reference range }\end{array}$} & \multicolumn{3}{|c|}{$\begin{array}{l}\text { standard } \\
\text { reference range }\end{array}$} & \multicolumn{3}{|c|}{$\begin{array}{l}\text { unbiased } \\
\text { reference rasuge }\end{array}$} \\
\hline
\end{tabular}

the six diagnostic groups. A good sensitivity is found for those having initial stages of Meniere's disease and for BPPD patients. Because with all of the approaches all controls scored normal, the specificity with respect to the controls remains 1.00 , and so does not influence the choice of the sensitivity criterium.

The specificities regarding non-patients with respect to a specific diagnosis are given

Table 8-8. Sensitivity and spectificity (regarding non-patients with respect to specific diagnosis) per diagnostic group and per

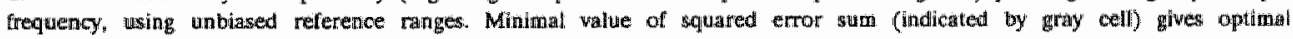
comientration.

\begin{tabular}{|c|c|c|c|c|c|c|c|c|c|c|c|c|}
\hline & \multicolumn{4}{|c|}{ sensitivity } & \multicolumn{4}{|c|}{ specincily } & \multicolumn{4}{|c|}{$\begin{array}{l}\text { snm of } \\
\text { squared deviations } \\
\text { from unity }\end{array}$} \\
\hline & $\begin{array}{l}0.16 \\
\mathrm{~Hz}\end{array}$ & $\begin{array}{l}0.41 \\
1 H z\end{array}$ & $\begin{array}{l}0.53 \\
\mathrm{~Hz}\end{array}$ & $\begin{array}{l}1.15 \\
\mathrm{~Hz}\end{array}$ & $\begin{array}{l}0.16 \\
H x\end{array}$ & $\begin{array}{l}0.41 \\
\mathrm{~Hz}\end{array}$ & $\begin{array}{l}0.53 \\
\mathrm{~Hz}\end{array}$ & $\begin{array}{l}1.15 \\
H x\end{array}$ & $\begin{array}{l}0.16 \\
\mathrm{~Hz}\end{array}$ & $\begin{array}{l}0.41 \\
\mathrm{~Hz}\end{array}$ & $\begin{array}{l}0.53 \\
H x\end{array}$ & $\begin{array}{l}1.1 .5 \\
12\end{array}$ \\
\hline 1. Meniere & 0.25 & 0.75 & 100 & 0.50 & 0.68 & 0.55 & 0.70 & 0.80 & 0.66 & 0.27 & 0.09 & 0.29 \\
\hline 2. compensiatled & 9.38 & 0.38 & 0.38 & 0.13 & 0.70 & 0.50 & 0.65 & 0.75 & 0.48 & 0.64 & 0.51 & 0.83 \\
\hline 3. encompensated & 0.31 & 0.54 & 0.23 & 0.15 & 0.69 & 0.54 & 0.60 & 0.74 & 0.58 & 0.42 & 0.75 & 0.78 \\
\hline 4. whiptash & 0.51 & 0.60 & 0.50 & 0.50 & 0,74 & 0.55 & 0,68 & 0.84 & 0.32 & 0.36 & 0.35 & 0.27 \\
\hline 6. BPPD & 0.33 & 0.67 & 0.33 & 0.00 & 0.69 & 0.53 & 0.64 & 0.76 & 0.54 & 0.33 & 0.57 & 1,06 \\
\hline $7_{4}$ unknown & 0.25 & 0.50 & 0.25 & 0.25 & 0.65 & 0.52 & 0.64 & 0.77 & 0.66 & 0.48 & 0.69 & 0.61 \\
\hline
\end{tabular}


in table 8-8, using the unbiased reference ranges. Also the sensitivities are here given. The best combination of sensitivity and specificity is found by choosing the least squared error sum (the deviance from unity is considered to be the error). Note that here the specificity will decrease if the sensitivity is increased by using the "inclusiveor" criterium.

The group best diagnosed with the gain contrast consists of those having initial stages of Meniere's disease (VVIT evaluated at the purely visually delivered frequency of $0.53 \mathrm{~Hz}_{\text {; }}$ sensitivity is 1.00 , specificity regarding other vestibular patients and controls is 0.70 ). And next are again those having Meniere (VVIT evaluated at the chairdelivered frequency of $0.41 \mathrm{~Hz}$; sensitivity is 0.75 , specificity is 0.55 ), and the whiplash patients (VVTT evaluated at the highest, chair-delivered frequency of $1.15 \mathrm{~Hz}$; sensitivity is 0.50 , specificity is 0.84 ). This latter confirms our initial impression that the whiplash patients distinct themselves from all others at the highest frequency, which was the reason that more of them were referred to us.

Remarkably, the groups for which the gain contrast has the best combinations of sensitivity and specificity (namely, those having initial stages of Meniere's disease, and the whiplash patients), those groups were only suggestively different from the controls according to MANOVA ( $p=0.099$ and $p=0.053$, respectively; table 8-5). This discrepancy might be caused by the differences in method of analysis: while MANOVA uses the inter-subject as wel] as the intra-subject variances of both patients and controls, the calculation of sensitivity and specificity is based on reference ranges, for which only the inter-subject variance of the controls was used. So high inter-subject variances for the controls and high intra-subject variances for both controls and patients may diminish the significance of the found differences of the mean for MANOVA, but leaves the sensitivity and specificity unaffected. In fact, different variances for controls and patients violates the MANOVA assumption of equal variances, which may make results even more different.

The fact that the gain contrast is rather insensitive can have various causes. The most obvious one is, that our concept of a SP system working on top of a compensating VOR, might be wrong. Other ones include the following. Half a year after this study had ended, it was noted that the rotatory chair responded less to the steering signals than it had done at the start. It is not known when that had begun, but if it had been during this study, stimuli would have been less intense and the SP upper limit would not have been surpassed as much as we intended. However, this would mean that the earlier patients would score more deviant than the latter ones, and no such a time effect could be found. Neither could such an effect be found for the controls, who where also measured at different times within the study period. As a third possibility, the total stimulus velocity might have been chosen too low. We calculated with maximum velocities, but, using a sum-of-sines stimulus, that maximum velocity was reached only a very small fraction of the time. And so the SP system could compen- 
sate much more than we initially recognized. As a fourth possibility, it might be that frequency-identifying mechanisms (predictors) interfere in an unknown way. This implies that an interaction between diagnostic group and stimulus frequency would have been found to be significant, but -except for the whiplash patients- no such an interaction was found (table 8-5). Finally, it might be that the diagnostic groups were composed of real patients and false positives (of the standard tests). Low sensitivities for the VVIT can result from some subjects scoring consistently in a normall way and others in a deviant way, or from all subjects scoring inconsistently for the various frequencies. To assess this, the individual number of deviant test re-

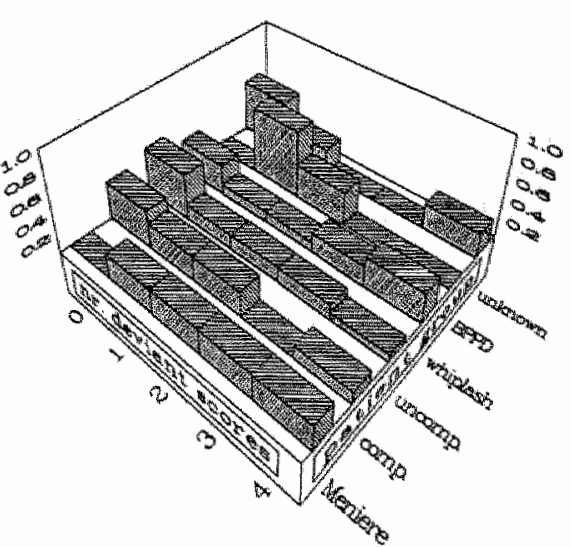

Fig. 87. Histograms per diagnositic group show that only the whiplash patients and those with unknown causes might include normal reacting subjects and typical patients. (horizontally: number of devarti scores, vertically: normalized number of subjects). sults (for the various frequencies) was rated per diagnostic group, and these rates were displayed in histograms (figure 8-7).

The histograms are placed behind each other from left to right for easy comparison of the diagnostic groups, and they are normalized, making group sizes unimportant. If within a diagnostic group a sub population scored consistently deviant and another subpopulation scored consistently normal, then the histogram would show two peaks, one at the lower classes and one at the higher classes. Alternatively, when all subjects scored inconsistently, there would be one (possibly broad) peak at the middle classes. Only the groups formed by whiplash patients and by vestibular patients for whom the cause is unknown, seem to consist of normal and deviant subgroups.

\subsection{Conclusion}

When considering group means of VVIT gain contrast in a multivariate way, three groups of patients can be found to differ significantly $(p<0.05)$ from the controls: $I$ those with a well compensated peripheral vestibular dysfunction, 2 those with a partly compensated vestibular dysfunction, and $\underline{3}$ those with whiplash trauma. And two groups (Meniere patients and BPPD patients) might do so $(0.05<\mathrm{p}<0.10)$. No deviations were detected for patients for whom the causes of their vertigo complaints are unknown.

When using a reference range that is based solely on the controls, gain contrast is 
sensitive for Meniere patients and BPPD patients only (sensitivity of 1.00), and poorly so for the other groups (sensitivities between 0.50 and 0.70 ). Specificity is 1.00 with respect to controls.

When using the response at a single frequency for differential diagnosis, VVIT gain contrast is suitable for Meniere patients and whiplash patients only (sensitivity of 1.0 . and speciffity of 0.70 , and sensitivity of 0.50 and specificity of 0.84 , respectively).

\subsection{Appendix.}

Whether responses of the diagnostic groups are Normally distributed, was checked by means of Normal probability plots, in which the expected Normal values are plotted against the actual values. Deviations from a straight diagonal line indicate deviations from Normality, which can be seen clearer after detrending (Le., subtracting the trend). When a distribution is found to be non-Normal, two strategies can be followed. If all data are considered equally important, then transforming them can be tried. Alternatively, some data can be considered outliers and can be excluded.

A Normalizing Box-Cox transformation is given by (Sokal \& Rohlf, 1981, p 423-427):

$$
\begin{aligned}
& \begin{cases}Y^{\prime}=\frac{Y^{\lambda}-1}{\lambda} & (\lambda \neq 0) \\
Y^{\prime}=\ln Y & (\lambda=0)\end{cases} \\
& \text { Y observed values } \\
& Y^{\prime} \text { transformed data } \\
& \lambda \text { parameter }
\end{aligned}
$$

with the parameter $\lambda$ obtained by maximizing the log likellihood function (Sokal \& Rohlf, 1981, p 424):

$$
\begin{aligned}
& L=-\frac{v}{2} \cdot \ln s_{T}^{2}+(\lambda-1) \cdot \frac{v}{n} \cdot \Sigma \ln Y \\
& \text { n number of observations } \\
& v \text { degrees of freedom: } v=n-1 \\
& Y \text { observed values } \\
& 1 \text { value to be maximized } \\
& S_{\text {I }}^{*} \text { estimate of variance of transformed data } \\
& \lambda \text { sought value for parameter of } \mathbb{B} 0 x-\operatorname{Cox} \text { corrective } \\
& \text { data transformation }
\end{aligned}
$$

Because contrast values and phase shifts can be negative, they are first transformed by adding their most negative value.

A separate value for the parameter $\lambda$ has to be calculated for each sub group, but 

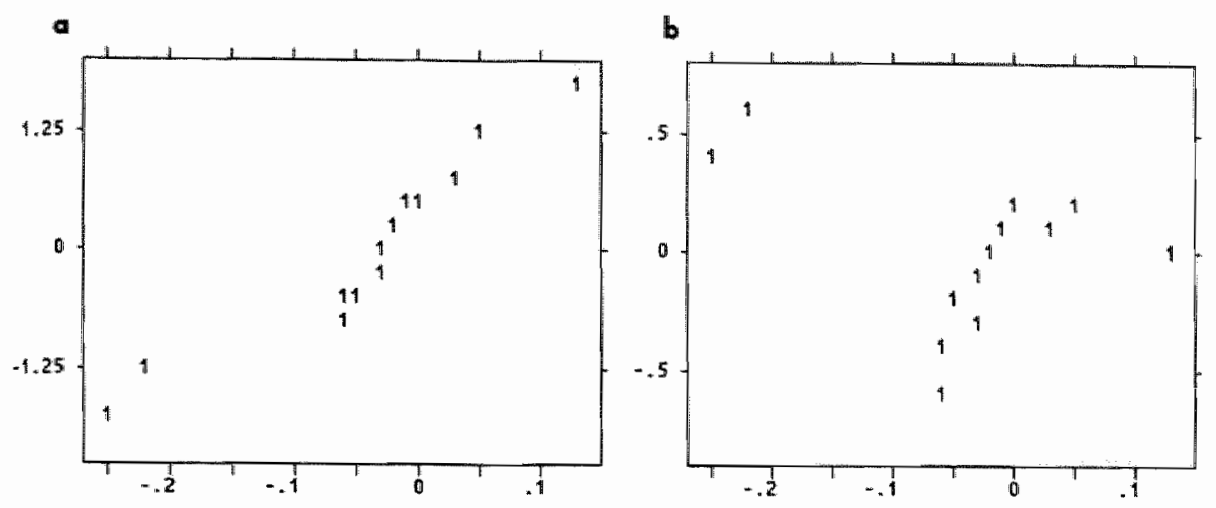

Fig. 8.8. Example of identification of outliers. an Nonmal probability plot of gain contrast data from partly conapensatiod peripheral vestibular dysfunction patients (group 3) for $1.15 \mathrm{~Hz}$ b. same as a, but dietrended: value below -(1)2 are rejected beatese they do not follow the Nornal distribution and lie separately from the bulk of dala.

only one transformation should be done on all data. The more sub groups need the same transformation, the more probable it is that the non-Normality is caused by inappropriate scaling.

Outliers were identified in detrended Normal probability plots, in which deviations from Normality are plotted against observed values. To be designed an outlier, an observed value should be far from the Normal distribution and be clearly separated from the bulk of data as well. Figures 8-8 and 8-9 show that this method rejects less data than simple thresholding. In figures $8-8 \mathrm{a}$ and $8-8 \mathrm{~b}$ the values below 0.2 are rejected, while in figures $8-9 \mathrm{a}$ and $8-9 \mathrm{~b}$ all vallues, including the ones below 0.2 , are accepted because they follow the Normal distribution.

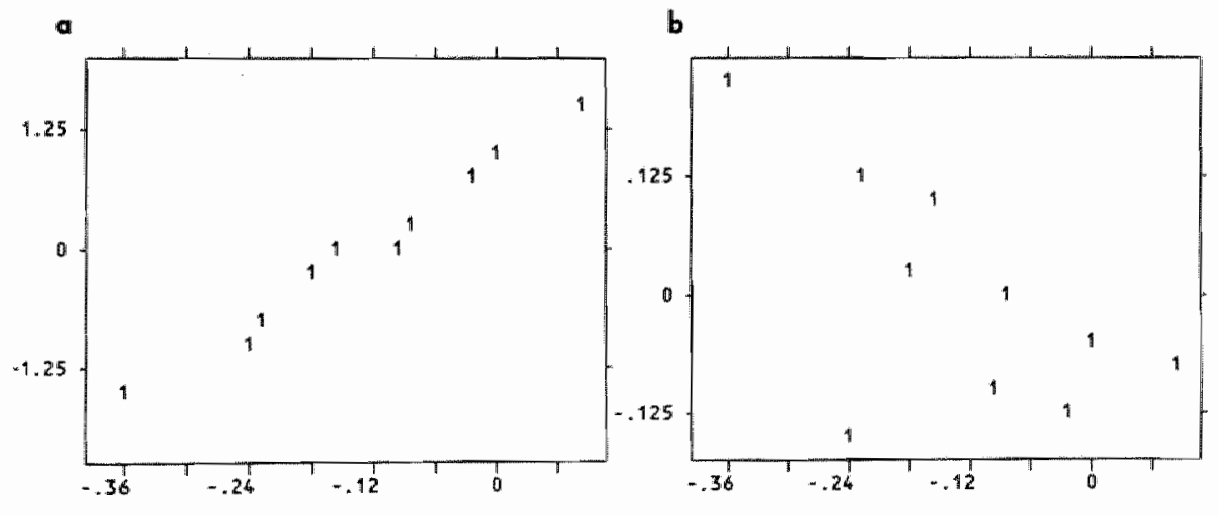

Fig. 84. Example of retaining low values. a Normal probability plot of gain contrast data from whiptash patients lor 1.15 Hz. b. same as an but detrended values below -0.2 are retained because they follow the Normall diatribution. 
In this study, a minimum distance of 0.5 units to the Normal distribution was use while the separation of potential outliers and the bulk was judged on sight. 


\section{Effect of asynchronous visual stimulation on the Vestibulo-Ocular Reflex phase shift of patients, compared to that of normals.}

\subsection{Abstract.}

A vestibular function test (named Visuo-Vestibular Interaction Test: VVIT) is presented, in which a visual target may move asynchronously with a rotatory chair. The phase shifts of the responses and their individual differences for two modes of stimulation ( $l$ sum-of-sines chair movement with an earth-fixed visual target: eftVVI, 2 sum-of-sines chair movement with an independent sinusoidal target movement: $\mathrm{mtVVI}$ ) are statistically analyzed for six groups of different types of vestibular patients (totalling 42 subjects) and a control group (6 subjects). Patients were diagnosed using anamneses and standard tests. Here, only the phase shifts are considered, the results of analyzing the gains and the coherences are reported elsewhere (chapters 8 "Effect of asynchronous visual stimulation on the VestibuloOcular Reflex gain of patients, compared to that of normals" and 10 "Effect of asynchronous visual stimulation on the Vestibulo-Ocular Reflex coherence of patients, compared to that of normals").

The whiplash patients and the patients having centrally well compensated peripheral vestibular dysfunctions differed significantly $(p=0.037$ and $p=0.054$, respectively) from the controls in their phase shift response to mtVVI stimulation, while the other groups did not $(\mathrm{p}>0.100)$. After taking the individual differences between responses to $\mathrm{mtVVI}$ and eftVVI stimulation, two small groups differed significantly from the controls: those having benign paroxysmal positioning dizziness (BPPD; $\mathrm{p}=0.035$ ) and those having the syndrome of Meniere $(p=0.042)$.

The best combinations of sensitivity \& specificity (with respect to related groups) were found for the Meniere and the BPPD patients with mtVVI stimulation $(0.75$ \& 0.73 and $0.67 \& 0.78$, respectively), and for the whiplash patients with the difference of mtVVI and eftVVI stimulation (0.60 \& 0.79 ).

Specificity with respect to controls only, was 1.00 for all four frequencies and for both reference ranges.

\subsection{Introduction.}

A full introduction of the VVIT was given earlier (chapter 5: "Effect of asynchronous visual stimulation on the Vestibulo-Ocular Reflex gain of normals"), so here only the essentials will be repeated.

In order to elude the variation inherent to measuring the vestibulo-ocular reflex (VOR) in the dark, a visual target is presented. The usual ways in which the resulting 
visuo-vestibular interaction (VVI) is studied, (namely, by offering either an earth-fixed target or a chair-fixed target) allow, but may not require, the two systems to interact. To force an interaction between smooth pursuit (SP) and VOR, we use a visual target which moves asynchronously with the chair. Predictive effects are reduced by having the chair move in a pseudo random (sum-of-sines) way. As both visual target movement and chair movement are below the SP upper limit, we expect normals and patients to perform equally well to either one of them. But because the combination is above the SP upper limit, we expect patients to perform worse than normals to the combined stimulus, assuming that they will overburden their SP system in an effort to compensate for the insufficient vestibular function. By subtracting the visuo-vestibular interaction with an earth-fixed target (eftVVI; i.e., visually enhanced VOR) stimulus and the response to it, from the visuo-vestibular interaction with an asynchronously. moving target (mtVVI) stimulus and the response to it, the wisual components are obtained. It is hypothesized that the vestibular function is reflected by the ratio of the smooth and saccadic eye movements (and thus by the gain of the median-filtered response). Different phase shifts for normals and patients and for eftVVI and mtVVI might be expected in relation to the complexity of the stimulus and the type of neural processing it is subjected to.

\subsection{Material and methods. 9.3.1 Subjects.}

Data were obtained from the subjects who were described earlier (see chapter 8 ). In short, 42 patients (median age: 45 ) and 6 controls (median age: 30 ) were examined. The patients felt in 6 categories: those having initial stages of Meniere's syndrome (4), those having peripheral vestibular dysfunctions, centrally well compensated (8) and centrally partly compensated (13), whiplash victims (10), BPPD patients (3), and those for which the causes of their complaints are unknown (4). They were diagnosed by an experienced vestibulologist (dr. Kingma), using anamneses and results of standard tests (see below). All subjects were tested without optical corrections.

\subsubsection{Experimental set-up.}

The experimental set-up was as reported previously (chapter 5), and so only a brief description follows here. Subjects sat in a rotary chair moving in a pseudo random way (sum-of-sines: $0.16,0.41$, and $1.15 \mathrm{~Hz}_{*}$ each with a maximum velocity of $10 \%$ ), 
while watching a dot which was either earth-fixed or moving independently $(0.53 \mathrm{~Hz}$, with a maximum velocity of $32 \%$ ). The eye position was measured using infra red reflection, and the head velocity was monitored with an angular rate sensor. Eye position, head velocity, and visual target position were sampled at a $50 \mathrm{~Hz}$ frequency for 125 seconds by a 12 bit analog-to-digital (AD) converter (the first two after being filtered analogously with a cut-off frequency of $10 \mathrm{~Hz}$ ) and were digitally stored for offline analysis.

Standard tests consisted of calorization $\left(30^{\circ}\right.$ Celsius and $44^{\circ}$ Celsius, in the order right cold, left cold, left warm, right warm), torsion in the dark $(0.1 \mathrm{~Hz}$ frequency, 50 '/s peak velocity), a smooth pursuit test $\left(0.29 \mathrm{~Hz}\right.$ frequency, $15^{\circ}$ amplitude), and an optokinetic test $(10,20,30$, and $40 \% / \mathrm{s}$ velocity, both horair and anti-horair). Left and right horizontal eye position was measured by electro-oculography, and was sampled at $100 \mathrm{~Hz}$.

\subsubsection{Digital signal analysis.}

Processing and analysis of the signals was as reported before (see chapter 5). In summary, position data were digitally differentiated to velocities, after which the total stimulus was combined from visual target movement and head movement. In order to calculate the phase shift over the same time length as gain and coherence were, the digitally stored signal was resampled by taking every third data point, so that the total time length fitted in the 2048 points available for fast Fourier transformation (FFT). The resulting lower sampling rate brought as an additional advantage a higher frequency resolution (at the cost of a lower frequency maximum, which was unimportant for the VVIT). Fast components (i.c., forward saccades, eye blink artefacts) in the response signals were removed or attenuated by using a median filter. The resampling made a different width of the median filter optimal than was used for the gain and coherence calculations, because the filter effect depends on number of data points as well as on time length. The used width was $0.35 \mathrm{~s}$ (nearest higher odd number of resampled data points is 7 for a recording time of $125 \mathrm{~s}$ ). The phase shift values at the response frequencies were extracted automatically from the spectrum, using preset criteria.

The values shown in the graphs are corrected for phase shifts introduced by the measurement system, assuming that for frequencies up till $0.53 \mathrm{~Hz}$ the combination of the SP system and the predictor will dominate for a normal person in responding to single-sine eftVVI and SP stimuli (see chapter 6 "Effect of asynchronousl visual stimulation on the Vestibulo-Ocular Reflex phase shift of normals"). 


\subsubsection{Statistical analysis.}

Because systematic errors are unimportant for comparing groups measured with the same equipment, the uncorrected values have been used to test the differences statistically. Statistical analysis was as reported earlier (see chapter 8). After removing univariate non-Normal outliers, a multivariate analysis of variance (MANOVA) was done to assess the significances with which various factors correlated with the observed differences of the means. Four influences were analyzed: the within-subject factor stimulus frequency, the between-subjects factors sex and diagnosis, and the covariate age. The distribution of the residuals was used to detect multivariate outliers.

Sensitivity was calculated per diagnostic group for two reference ranges (a standard one (Albert \& Harris, 1987) and an unbiased one (Royston \& Matthews, 1991); see chapter 5 ) in three ways ( 1 using each subject's best single result, 2 lumping together intra-subject and inter-subject results, 3 labelling subjects as patients if any of their intra-subject results was outside the reference ranges). Specificity was calculated regarding controls only, as well as regarding other diagnostic groups and controls.

\subsection{Results and discussion.}

The VVIT consists of chair movements with a visual target that is earth-fixed, and with one that moves asynchronously. And so three sets of data are obtained: one resulting from the first stimulus condition (eftVVI), one resulting from the second stimulus condition (mtVVI), and one resulting from taking the individual differences of the first two sets (calculating the means of the individual differences gives the same values as taking the differences of the means, but produces other variances).

For all three sets, the distributions of the responses were checked on Normality by means of Normal probability plots. In case of deviations, two strategies are possible for Normalizing the data. If one considers all data equally important, then transforming the data can be tried. Alternatively, some data can be considered outliers and can be excluded.

To see whether the first strategy was feasible, values for the parameter $\lambda$ for corrective Box-Cox transformations (see the appendix of chapter 8) were calculated for each frequency-group combination. The more combinations need similar transformations, the more probable it is that the observed deviations from Normality are caused by inappropriate scaling. If no single transformation will do for all com- 
binations, then the second strategy has to be used. If in a Normal probability plot a value deviated 0.5 units or more from the Normal distribution and was lying distinct from the bulk of data, it was considered to be an outlier, eligible for removal. The responses of that same subject to the other stimulus frequencies were then also removed, irrespective whether they fitted in the Normal distribution.

The Normal probability plots strongly suggest that diagnostically defined group 3 consists of two differently reacting subgroups. This is studied in more detail in part 1 "Group 3 might consist of two sub groups" of chapter 13 "General discussion". Because the distinction is suggested by the current VVIT data only, and not by anamneses or standard test outcomes, it would be inappropriate to split the group and statistically test the subgroups.

To assess the clinical usefulness of a test, its sensitivity (i.e., the ratio of found and actual patients) and its specificity (i.e., the ratio of found and actual non-patients) are calculated (e.g., Sacket et al, 1985, p 69-72). This is straightforward in case of a univariate test and one type of patients, but becomes a little more complicated in our case. The current VVIT produces 26 results' $^{3}$, and in the current study the results of six types of patients ${ }^{2}$ are analyzed. Of course, all the data generated by the VVIT should be used to determine whether a subject is a patient, and if so, what type of patient he is. This can be done by combining the results with weights as found by cluster analysis (see chapter 11 "Discriminating power of VVIT for type of pathology, compared to those of standard tests"). With these end results the sensitivity and specificity can be calculated in the usual way. Nevertheless, it is also interesting to see whether the univariate results alone would allow us to draw valid conclusions, as that would greatly simplify diagnosis. Having various results, one may give the best sensitivity for a single variable-frequency combination (which can be different combinations for various groups of patients), the average sensitivity (i.e., all positive test outcomes, divided by the product of the number of outcomes per test times the number of patients), or the "inclusive-or" sensitivity ( $i . e$, the sensitivity when a subject is considered to be a patient if any of the test results deviates significantly from those of the normals). And one may give the specificity regarding controls only (i.e., no patients for any type of vestibular dysfunction), or for non-patients with respect to a specific type of vestibular dysfunction (and thus including other vestibular patients as well as controls).

\footnotetext{
26. VVIT results: efVWI phase shift and efVWL coherence, both for three frequencies, and gain contrasi, miVVI phase shift, phase shift difference, miVVI coherence, and coherence contrast, each for four frequencies.

${ }^{2}$ Stix rypes of patients: initial stizes of Meniene"s disease, centrally well compensated peripheral vestibular dystiunction, centrally poorly compensated peripheral westibular dysfunction, whiplash trauma, benign paroxysmal positioning dizziness, vestibular complaints from unkmown origin.
} 


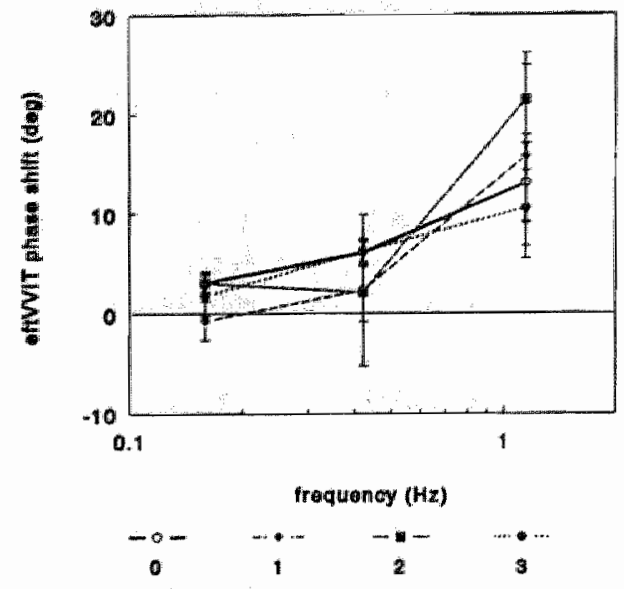

Fig 9-1. eft VVIT phtiase shift depending on stimulus frequency for controls (O) and not significantly different patierts (4 init. Meniere $=$ = well comp. periph. vest. dysf, $=$ poorly comp periph. vest. dyst.)

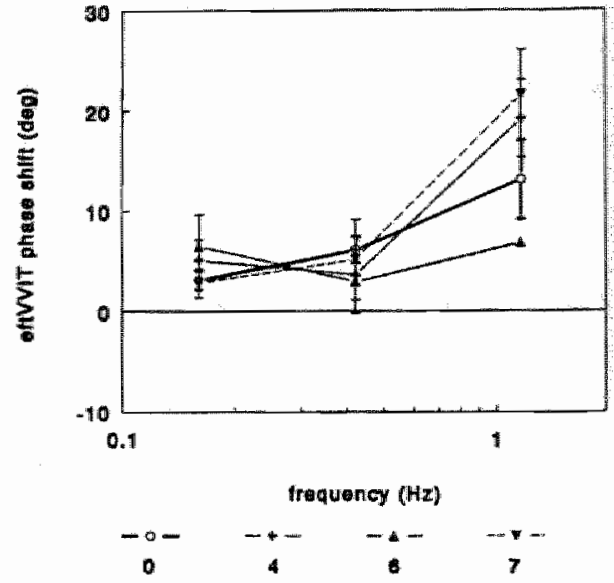

Fig. 92. eftWT phase shift deperding on stimulusi frequency for contrals ( $O$ ) and not significantly difterent patients $(+=$ whiplash, $4=$ benign paroxysm posit. vertigo, $=$ unknown'

\subsubsection{Visuo-vestibular interaction with an earth-fixed visual target.}

In figures 9-1 and 9-2 the corrected phase shift values for visuo-vestibular interaction with an earth-fixed target (eftVVI) are depicted per group and per chair-delivered stimulus frequency. The patient groups are distributed over two figures for clarity only, none differed significantly from the controls (see below). Conform the statistical
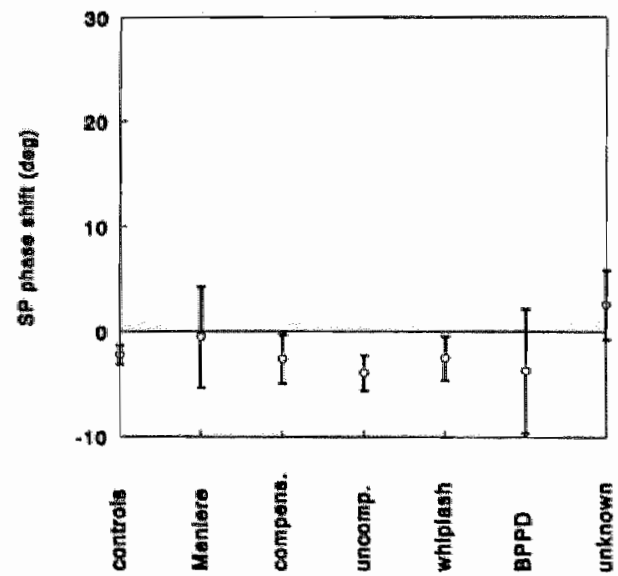

10. 9-3. Phase shifts resulting from single-sine $(0.53 \mathrm{~Hz})$

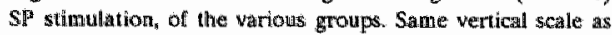
in figures $9-1$ and $9-2$. analysis to be described below, outlying values are excluded from the means and standard errors. Most patient groups had a sharper dip at $0.41 \mathrm{~Hz}$ than the controls, but due to their variations, those differences are statistically insignificant. Using the same vertical scaling, figure 9-3 shows the phase shifts resulting from single-sine $(0.53$ $\mathrm{Hz}$ ) SP stimulation. The patient groups do not appear to have a phase shift that differs from that of the controls. The fact that the corrected mean for the controls is not zero, might indicate that the one normal subject used to derive the correction predicted the single-sine stimulus better than on average, or that the equipment 
Table 9-1. Values for the parameter $\lambda$ of the Box-Cox transformation for various frequency - group tombinations, Bold printed values indivate combinations that deviate 0.5 uning or mone from the Normal distribution.

\begin{tabular}{|c|c|c|c|}
\hline diagnosis & $\begin{array}{l}0.16 \\
\mathrm{~Hz}\end{array}$ & $\begin{array}{l}0.41 \\
H z\end{array}$ & $\begin{array}{l}1.15 \\
\mathrm{~Hz}\end{array}$ \\
\hline O. oonurols & -3.5 & -32 & -13 \\
\hline 1. initial stages of Meniere's syndrome & 15.5 & 5.0 & -3.5 \\
\hline 2. peripheral vestibular dysfunction ${ }_{2}$ centrally well compensated & -32 & 9.6 & 2.4 \\
\hline 3. peripheral vestibular dystunction, centrally poorly compensated & 0.40 & -28 & 23 \\
\hline 4. whiplash trauma & 2. 1 & 25 & 1.3 \\
\hline 6. benign paroxysmal positioning dizziness & $-3,55$ & .1 .3 & +3.0 \\
\hline 7. vestibular complaints from unknown causes & 28 & 8.0 & 3.1 \\
\hline
\end{tabular}

had been altered between the end of the study and the later made checks on phase shifts introduced by the measurement system.

The responses to the chair-delivered movements appeared to be non-Normal in Normal probability plots. Values for the parameter $\lambda$ for corrective Box-Cox transformations are given in table 9-1 (bold printing indicate combinations deviate severely). Their variation suggests that the observed deviations from Normality are not caused by inappropriate scaling, and so individual outliers were sought. Using the above mentioned criteria, resulted in detecting two outliers (table 9-2). These were excluded together with the results for the other frequencies of those subjects and subsequent analysis was done on the remaining set.

The overall testing (table 9-3) estimated $(-2.4 \pm 2.1) \times$ age to be the effect of age on the eftVVIT phase shift, which is insignificant $(\mathrm{p}=0.251)$.

Table 9-2 Outlying resporse values for wariout frequency * group combinations. Criterion was deviating 0.5 units or mone from the Normal distribution as well as lying distinch trom the bulk of data-

\begin{tabular}{|c|c|c|c|}
\hline diagnosis & $\begin{array}{l}0.16 \\
\mathrm{~Hz}\end{array}$ & $\begin{array}{l}0.41 \\
1-1 z\end{array}$ & $\begin{array}{l}1.55 \\
\mathrm{~Hz}\end{array}$ \\
\hline 0. controls & 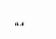 &. & .. \\
\hline 1. inillial stage of Meniere's syndrome & $"$. & ". & ." \\
\hline 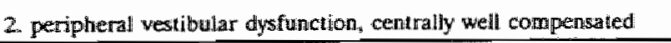 & . & -16 & .. \\
\hline 3. peripherall vestibuiar dysfunction, centrally poorly compensated & " & ." & -19 \\
\hline 4. whiptash trauma & .. & $\cdot n$ & 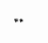 \\
\hline 6. beiaign paroxysmal positioning dizxiness & $\therefore$ & ." & 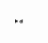 \\
\hline 7. westibular complaints from unknown causes &. & $\therefore$ & ..4 \\
\hline
\end{tabular}




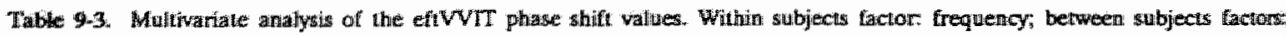
sex diagnessis; cotwariatite age.

\begin{tabular}{|c|c|c|c|c|c|}
\hline \multicolumn{6}{|c|}{ significance of independent variables $(p=\ldots)$} \\
\hline age & $\begin{array}{l}\text { diagnosis } \\
\text { (after inveraction) }\end{array}$ & (after interaction) & $\begin{array}{l}\text { interaction of } \\
\text { sex and frequency }\end{array}$ & $\begin{array}{l}\text { interaction of } \\
\text { diagr. and frem. }\end{array}$ & $\begin{array}{l}\text { effect of fineq. } \\
\text { (after interactions) }\end{array}$ \\
\hline 0,231 & 0.551 & 0.31 & 0.931 & 0.711 & $<0,00005$ \\
\hline
\end{tabular}

Because overall testing failed to show any significant (or even any suggestive) difference between groups, no pairwise testing is allowed. The lack of difference supports the initial assumption that controls as well as patients are able to track the target during the effVVIT (presumably because the velocity remains below the upper limit of the SP system). Assuming that not finding any significant difference in group means implies that individual values will be rather similar for patients and controls, they are not compared to a reference range.

\subsubsection{Visuo-vestibular interaction with an asynchronously moving visual target.}

In figures 9-4 and 9-5 the corrected phase shift values (mean \pm standard error) for visuo-vestibular interaction with a moving target ( $\mathrm{mtVVD}$ ) are depicted per group and

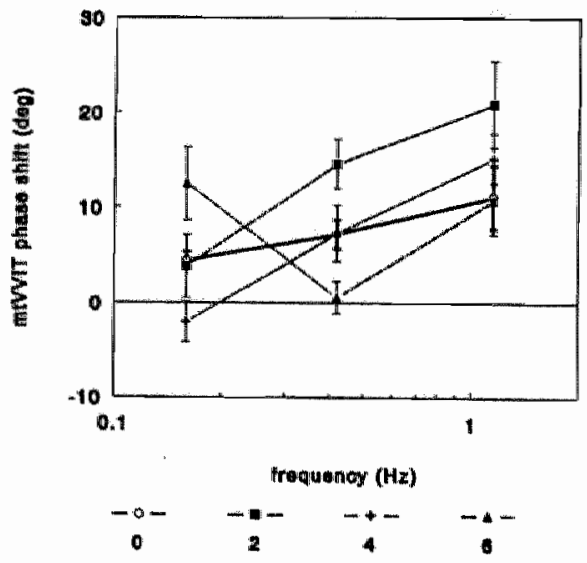

Fig. 94. miVIT phase shift depending on stimulus frequency for controls ( 0 ) and significantly different patients (w well comp. periph. vest. dysif, + whiplash ${ }_{*}=$ BPPD),

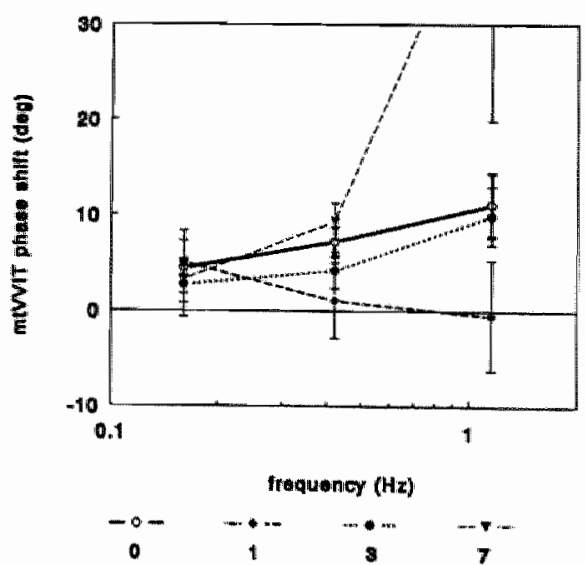

Fige 9-5. miVVT phase shift depending on stimuluis frequency for controls $(0)$ and not significantly different patients ( $=$ init. Meniere, $=$ incompl. comp. periph. vest. dysf., $v=$ unknown). 
per chair-delivered stimulus frequency. Conform the statistical analysis to be described below, the patient groups are divided in those who differed significantly from the controls, and those who did not, and outlying values are excluded. The two vestibular patient groups who differ significantly from the controls (those with well compensated peripheral vestibular dysfunctions, and those with benign paroxysmal positioning dizziness), both have a greater increase of the phase lead. The whiplash patients, however, have for the middle chair frequency $(0.41 \mathrm{~Hz})$ a zero phase shift but for both lower and higher chair frequencies a phase lead. Repeating the measurements with intermediate stimu-

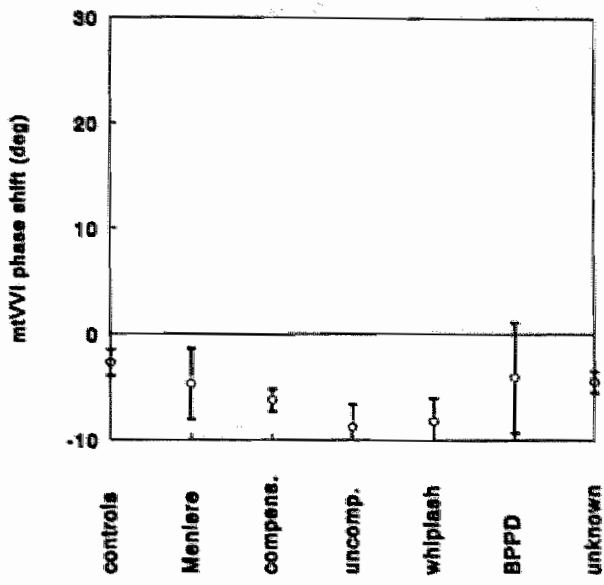

Fig. 9-6. Phase shifts resulting from singlewsine $(0.53 \mathrm{~Hz})$ wisual target movement, simultanous and asymchronous with the sum-of-sines chair movement. Same vertical scalling as figure 9-4 and 9-5. lus frequencies should elucidate whether this is a real effect. If so, then the closiness of the visual target frequency $(0.53 \mathrm{~Hz})$ might indicate that the whiplash patients use the SP system not only to track the visual target movement, but also let it influence responses to nearby chair frequencies. This can be tested by employing a different visual target frequency. The phase shift dip at $0.41 \mathrm{~Hz}$ for eftVVI stimulation (and so without a $0.53 \mathrm{~Hz}$ visual target movement) as depicted in figures 9-1 and 9-2, however, plead against the SP explanation.

Using the same vertical scaling, figure 9-6 shows the phase shifts at the frequency of the asynchronously moving visual target. Compared with the shifts resulting from SP

Table 9-4. Values for the parameter $\AA$ of the Box-Com transtormation for varions freguency * group combinations Bold printed wat ues indicate combinations that deviate 0.5 units or more from the Normal distribution.

\begin{tabular}{|c|c|c|c|}
\hline diagnosis & $\begin{array}{l}0.16 \\
\mathrm{Hiz}\end{array}$ & $\begin{array}{l}0.41 \\
\mathrm{~Hz}\end{array}$ & $\begin{array}{l}1,15 \\
\mathrm{~Hz}\end{array}$ \\
\hline 0. controls & 1.2 & -30 & 1.2 \\
\hline 1. injiall stages of Meniere's syndrome & -3.2 & $-3,3$ & 7.5 \\
\hline 2. peripheral vestibular dysfunction, cerirally well compensated & $0.4 \mathbb{1}$ & -3.3 & -3.1 \\
\hline 3. peripheral westibular dysfunction, centrally partly compensated & 6.9 & 5.5 & 22 \\
\hline 4. whilplasth trauma & 0.40 & -23 & 3.9 \\
\hline 6. benign parroxysmal pasitioning dizziness & -3.3 & 6.5 & 11 \\
\hline 7. vestibular complaints from unknown causes & 10.5 & 4.7 & -3.2 \\
\hline
\end{tabular}




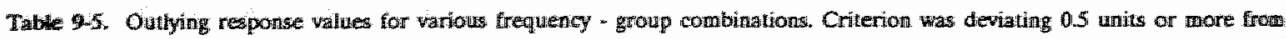

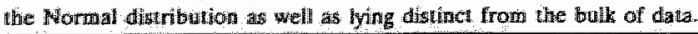

\begin{tabular}{|c|c|c|c|}
\hline diagnasis & $\begin{array}{l}0.16 \\
\mathrm{~Hz}\end{array}$ & $\begin{array}{l}0.41 \\
\mathrm{~Hz}\end{array}$ & $\begin{array}{l}1.15 \\
\mathrm{~Hz}\end{array}$ \\
\hline 0. controls &. & ." & \% \\
\hline 1. initial stages of Menere's syndrome & $\ldots$ & $\cdots$ & $\cdots$ \\
\hline 2. peripheral vestibular dystunction, centrally well compensated & .. & $\therefore$ & .. \\
\hline 3. peripheral vestibular dysfunction, centrally poorly compensated & -28 & .. & .. \\
\hline 4. whiplash trauma & .. & 53 & -66 \\
\hline 6. bentign paroxyamal positionirig dizziness & .. & .. &. \\
\hline 7. UnknOAH & .. & ... & .. \\
\hline
\end{tabular}

stimulation (figure 9-3: same actual target movement, but no chair movement), all groups have a slightly increased delay, which remains still well below the delay of $17^{\circ}$ expected for a visual processing time of $90 \mathrm{~ms}$. So apparantly the predictor is working, but perhaps an extra processing step is included when the head is moved too. It will be interesting to see if this increase in delay is fixed for all head movements, or if it varies with the randomness (and thus the unpredictability).

The responses to the chair-delivered frequencies appeared to be non-Normall in Normal probability plots. Values for the parameter $\lambda$ for corrective Box-Cox transformations are given in table $9-4$ (bold printing indicate combinations deviate severelly'). Their variation suggests that the observed deviations from Normality are not caused by inappropriate scaling and so individial outliers were sought. Using the above mentioned criteria resulted in detecting three outliers (table 9-5). These were

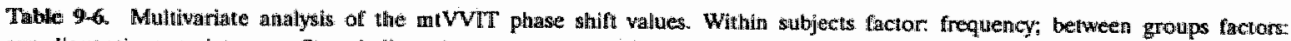
wer, diagnosis; covariate age. Stars indicate important group differences.

\begin{tabular}{|c|c|c|c|c|c|c|c|}
\hline & & \multicolumn{6}{|c|}{$\begin{array}{l}\text { significance of independent wariables } \\
\qquad(\mathrm{p}=\ldots)\end{array}$} \\
\hline & & age & $\begin{array}{l}\text { diagn. (after } \\
\text { interaction) }\end{array}$ & $\begin{array}{l}\text { sex (ather } \\
\text { interactioni) }\end{array}$ & $\begin{array}{l}\text { interaction } \\
\text { of sex and } \\
\text { freq. }\end{array}$ & $\begin{array}{l}\text { interaction } \\
\text { of diagn. } \\
\text { and freq. }\end{array}$ & $\begin{array}{l}\text { effect of } \\
\text { freq. (after } \\
\text { interactions) }\end{array}$ \\
\hline \multirow{2}{*}{$\begin{array}{l}\text { indtependent } \\
\text { variables } \\
\text { inchudted for } \\
\text { analysis }\end{array}$} & $\begin{array}{l}\text { age, digghosis, } \\
\text { sex, frequency }\end{array}$ & \multirow[t]{2}{*}{0,283} & $0.094(t)$ & 0.770 & 0.369 & $0.006+4$ & 0.105 \\
\hline & $\begin{array}{l}\text { diagnosis } \\
\text { frequency }\end{array}$ & & $0.050+4$ & $m$ & . & $0,009+4$ & 0,082 \\
\hline
\end{tabular}


Tuble 9-7. MANOVA reswits of pairwise comparison of mEVIT phase shitt walles from warious patient groups with as control

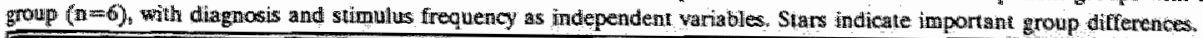

\begin{tabular}{|c|c|c|c|c|}
\hline \multirow[t]{2}{*}{ diagriosis } & \multirow{2}{*}{$\begin{array}{l}\text { number } \\
\text { of } \\
\text { members }\end{array}$} & \multicolumn{3}{|c|}{$\begin{array}{c}\text { sidnificance } \\
(p=2)\end{array}$} \\
\hline & & $\begin{array}{l}\text { diagnosis } \\
\text { (after } \\
\text { interaction) }\end{array}$ & $\begin{array}{l}\text { interaction of } \\
\text { disgnosis and } \\
\text { trequency }\end{array}$ & $\begin{array}{l}\text { frequency } \\
\text { (after } \\
\text { interaction) }\end{array}$ \\
\hline 1. ittitial stages of Meniere's syndrome & 4 & 0.138 & 0.465 & 0.773 \\
\hline 2. peripheral vestibular dysfunction, well compensaled & 8 & $0.054 x$ & 0.263 & 0.057 \\
\hline 3. peripheral vestibular dysfunction, poorly compensated & 12 & 0.723 & 0,792 & 0.025 \\
\hline 4. withiplasile trauna & 8 & 0.926 & $0.037 \times$ & 0.002 \\
\hline 6. benign paroxysmai positioning dizziness & 3 & 0.612 & $0.004 \times 3$ & 0.0005 \\
\hline 7. Mestibular complaints form unknown causes & 4 & 0.117 & 0.254 & 0.266 \\
\hline
\end{tabular}

\footnotetext{
* significant at $5 \%$ level

+ significant at lo level
}

excluded together with the results for the other frequencies of those subjects. The overall testing (table 9-6) estimated $(-3.1 \pm 2.8) \times$ age to be the effect of age on the mtVVIT phase shift, which is insignificant $(p=0.283)$. Neither did sex influence results significantly ( $p=0.369$ for the interaction of sex and frequency, and $p$ $=0.770$ for the remaining effects of sex).

Because overall testing showed a (highly) significant difference between groups ( $\mathrm{p}=$ 0.009 for the interaction of diagnosis and stimulus frequency, and furthermore $p=$ 0.050 for the remaining effects of the diagnosed causes), each patient group separately may be compared with the controls.

The results of pairwise testing are shown in table 9-7.

From table $9-7$ can be seen that compared to the controls, groups 4 and 6 react in a significantly ( $p=0.037$ and $p=0.004$, respectively) different way to the stimulus frequencies, but do not differ in overall responses. Comparing the two deviant groups among themselves, shows them to differ from each other as well (table 9-8), and so they may not be combined. All other groups do not distinct themselves from the controls. But again, they differ from each other and may not be combined. In conclusion, two groups of patients (those with whiplash trauma and those with benign paroxysmal positioning vertigo) produce different clusters of deviant miVVIT phase shift values, and the other five groups (those in the initial stages of Meniere's syndrome, those with well compensated or incompletely compensated peripheral vestibular dysfunction, those for whom the causes of their vertigo complaints are unknown, and those with 


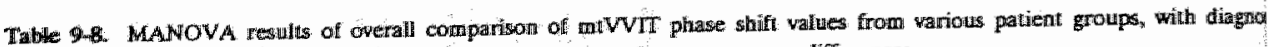
and timulus troquency andependent varibbles stars indicate inporiant group differences.

\begin{tabular}{|c|c|c|c|}
\hline \multirow[t]{2}{*}{ diagnowtic groups } & \multicolumn{3}{|c|}{$\begin{array}{c}\text { signifreance } \\
(P=\ldots)\end{array}$} \\
\hline & $\begin{array}{l}\text { diagnosis } \\
\text { (after } \\
\text { intenction) }\end{array}$ & $\begin{array}{l}\text { Interaction of } \\
\text { dilignosis and } \\
\text { frequency }\end{array}$ & $\begin{array}{l}\text { frequency } \\
\text { (nfier } \\
\text { interaction) }\end{array}$ \\
\hline 4 and 6 & 0.7118 & $0.005+x$ & 0.010 \\
\hline $0,1,2,3$, and 7 & $0.008 \times t$ & $0.048 \star$ & 0.015 \\
\hline $0,1,7$ & $0.059(t)$ & 0.418 & 0.204 \\
\hline 1 and 7 & $0.085(t)$ & 0.435 & 0.403 \\
\hline
\end{tabular}

(t) suggestiwe, but not significant at $5 \%$ level

* signiflicant al $5 \%$ level

+ significant at 1 cho level

multiple causes) produce mtVVTT phase shift values within the normal limits, but probably differ from each other.

The clinical usefulness is assessed by calculating the sensitivities and specificities for the various diagnostic groups. Table 9-9 shows that using the unbiased reference

Table 9-9. Sensitivities of the VVTT for miVVI phase sthift only (four frequencies) and overall (all variable-frequency con:

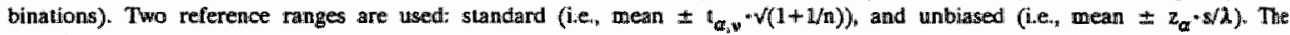
sensitiwity has been computted in three ways: the best single result per subject was used ("best"), no distinction was made between test resulus per subject and test results from various subjects ("aweraged"), and subjectsicre labeled patient, if any vest result was deviant ("inclusive-or). Specinciry with respect to controls is 1,00 for all three methods, and for both ranges.

\begin{tabular}{|c|c|c|c|c|c|c|c|c|c|c|c|c|}
\hline \multirow[t]{2}{*}{ dignosils } & \multicolumn{6}{|c|}{ maVVI phase shidt sensithvity } & \multicolumn{6}{|c|}{ owerall semsilivity } \\
\hline & t & $\begin{array}{l}\text { aver- } \\
\text { aged }\end{array}$ & $\begin{array}{l}\text { incl: } \\
\text { or }\end{array}$ & $b e s$ & $\begin{array}{l}\text { aver- } \\
\text { aged }\end{array}$ & $\begin{array}{l}\text { incl } \\
\text { or }\end{array}$ & best & $\begin{array}{l}\text { aver. } \\
\text { aged }\end{array}$ & $\begin{array}{l}\text { incl. } \\
\text { or: }\end{array}$ & best & $\begin{array}{l}\text { aver } \\
\text { aged }\end{array}$ & $\begin{array}{l}\text { indel. } \\
\text { or }\end{array}$ \\
\hline Meniere & 0.50 & 0.25 & 9.75 & 0.75 & 0.50 & 1.00 & 1.00 & 0.51 & 1.00 & 1.00 & 0.66 & 1.00 \\
\hline compensatted & 0.50 & 0.28 & 0.63 & 0.50 & 0.38 & 0.63 & 0.75 & 0.34 & 0.88 & 0.75 & 0.42 & 0,88 \\
\hline uncomperisated & 0.31 & 0.17 & 0.54 & 0.31 & 0.19 & 0.54 & 0.92 & 0.35 & 1.00 & 0.92 & $0,4: 2$ & 1.00 \\
\hline whiplasth & 0.50 & 0.28 & 0.80 & 0.60 & 0.38 & 0.80 & 1.00 & 0.51 & 1.00 & 1.00 & 0.60 & 1.00 \\
\hline BPPD & 0.33 & 0,17 & 1.00 & 0.67 & 0.42 & 1.00 & 1.00 & 0.42 & 1.00 & 1.00 & 0.52 & 1.00 \\
\hline \multirow[t]{2}{*}{ anknown } & 0.25 & $0: 13$ & 0.50 & 0.50 & 0.19 & 0.50 & 0.50 & 0.30 & 0.50 & 0.75 & 0.36 & 1.00 \\
\hline & \multicolumn{3}{|c|}{$\begin{array}{l}\text { standaind } \\
\text { reference range }\end{array}$} & \multicolumn{3}{|c|}{$\begin{array}{l}\text { unbiased } \\
\text { reference range }\end{array}$} & \multicolumn{3}{|c|}{$\begin{array}{l}\text { standard } \\
\text { reterence range }\end{array}$} & \multicolumn{3}{|c|}{$\begin{array}{l}\text { unbiased } \\
\text { reference range }\end{array}$} \\
\hline
\end{tabular}


Table 9-10. Sensithity and specificity (regarding non-patients with respect to specific diagnosis) per diagnostic group and per frequency, using standard reference ranges. Minimal value of squared ermor sum (indicated by gray cell) gives optinasl combination.

\begin{tabular}{|c|c|c|c|c|c|c|c|c|c|c|c|c|}
\hline & \multicolumn{4}{|c|}{ sensitivity } & \multicolumn{4}{|c|}{ specificity } & \multicolumn{4}{|c|}{$\begin{array}{l}\text { sum of } \\
\text { squared demations } \\
\text { from unity }\end{array}$} \\
\hline & $\begin{array}{r}0.16 \\
\mathrm{~Hz}\end{array}$ & $\begin{array}{r}0,41 \\
\mathrm{Hzz}\end{array}$ & $\begin{array}{r}0.53 \\
\mathrm{HIz}\end{array}$ & $\begin{array}{r}1.15 \\
\mathrm{Hiz}\end{array}$ & $\begin{array}{r}0.16 \\
\mathrm{~Hz}\end{array}$ & $\begin{array}{r}0.41 \\
\mathrm{~Hz}\end{array}$ & $\begin{array}{r}0.53 \\
\mathrm{~Hz}\end{array}$ & $\begin{array}{r}1.15 \\
\mathrm{H} z\end{array}$ & $\begin{array}{r}0.16 \\
\mathrm{~Hz}\end{array}$ & $\begin{array}{r}0.41 \\
\mathrm{~Hz}\end{array}$ & $\begin{array}{r}0.93 \\
H H L\end{array}$ & $\begin{array}{r}1.15 \\
112\end{array}$ \\
\hline 1.Meniere & 0.50 & 0.25 & 0.00 & 0.25 & 0.66 & 0.84 & 0.84 & 0.91 & 0.37 & 0.59 & 1.03 & 0.57 \\
\hline 2oompensalted & 0.50 & 0.25 & 0.13 & 0.25 & 0.68 & 0.85 & 0.85 & 0.93 & 0.36 & 0.59 & 0.79 & 0.57 \\
\hline 3.uncompensated & 0.31 & 0.15 & 0.23 & 0.000 & 0,63 & 0.83 & 0.89 & 0.86 & 0.62 & 0.75 & 0.60 & 1.02 \\
\hline 4. 4hiplasiti. & 0.50 & 0.30 & 0.20 & 0.10 & 0,68 & 0.87 & 0.87 & 0.89 & 0.35 & 0.51 & 0.66 & 0.82 \\
\hline 6.BPPD & 0.33 & 0.00 & 0.33 & 0.00 & 0.64 & $0: 82$ & 0.87 & 0.89 & 0.57 & 1.03 & 2.46 & 1.01 \\
\hline 7.unknown & 0.25 & 0.00 & 0.00 & 0.25 & 0.64 & 0.82 & 0.84 & 0.91 & 0.69 & 1.03 & 1.03 & 0.57 \\
\hline
\end{tabular}

ranges results in values that are generally better for the best single frequency and for the averaged sensitivities than when using the standard reference ranges. For the "inclusive or" sensitivities, there is almost no difference between using the two kinds of reference ranges. Comparing the $\mathrm{mtVVI}$ phase shift sensitivities with those calculated using all variable-frequency combinations, reveals that the first is one of the worst, for its sensitivites for all three categories (best single frequency, averaged, inclusive or) are the smallest. Only for the BPPD group is the "inclusive-or" mtVVI

Table 9-11 Sensitivity and specificity (regarding non-patients with respect to specific diagncsis) per diagnostic group and per

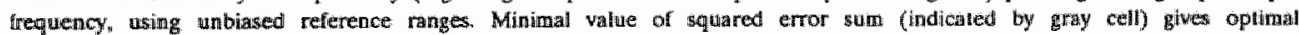
corrabination.

\begin{tabular}{|c|c|c|c|c|c|c|c|c|c|c|c|c|}
\hline & \multicolumn{4}{|c|}{ sensitivity } & \multicolumn{4}{|c|}{ specificity } & \multicolumn{4}{|c|}{$\begin{array}{l}\text { sum of } \\
\text { squared deviations } \\
\text { from wing }\end{array}$} \\
\hline & $\begin{array}{r}0.16 \\
1 \%\end{array}$ & $\begin{array}{r}0.41 \\
H z\end{array}$ & $\begin{array}{r}0.53 \\
\mathrm{Fiz}\end{array}$ & $\begin{array}{r}1.15 \\
1 \mathrm{~Hz}\end{array}$ & $\begin{array}{r}0.16 \\
1612\end{array}$ & $\begin{array}{r}0.41 \\
\mathrm{~Hz}\end{array}$ & $\begin{array}{r}0.53 \\
\mathrm{~Hz}\end{array}$ & $\begin{array}{r}1.15 \\
\mathrm{~Hz}\end{array}$ & $\begin{array}{r}0: 16 \\
112\end{array}$ & $\begin{array}{r}0.41 \\
H_{2}^{2}\end{array}$ & $\begin{array}{r}0.53 \\
\mathrm{Hl}\end{array}$ & $\begin{array}{r}\text { 4. } 15 \\
\mathrm{~Hz}^{2}\end{array}$ \\
\hline 1.Mentere & 0.50 & 075 & 0.50 & 0.25 & 0.59 & 0.73 & 0.77 & 0,89 & 0.42 & 0.14 & 0.30 & 0.58 \\
\hline 2.ocampensalled & 0.50 & 0.50 & 0.13 & 0.38 & 0.60 & 0.73 & 0.73 & 0.93 & 0.41 & 0.33 & 0.84 & 0.40 \\
\hline Auncompetrsated & 0.31 & 0.15 & 0.31 & 0.00 & 0.54 & 0.63 & 0.77 & 0.83 & 0.69 & 0.85 & 0.53 & 1.03 \\
\hline 4 whiplash & 0.60 & 0.50 & 0.30 & 0.10 & 0.63 & 0.74 & 0.76 & 0.87 & 0.30 & 0.32 & 0.55 & 0.83 \\
\hline GEPPD & 0.67 & 0.33 & 0.67 & 0.00 & 0.60 & 0.69 & $0.7 B$ & 0.87 & 0.27 & 0.5 .4 & 0.16 & 1.02 \\
\hline Tumbnown & 0.50 & 0.00 & 0.00 & 0.25 & 0.59 & 0.646 & 0.73 & 0,89 & 0.42 & 1.12 & 1.07 & 0.58 \\
\hline
\end{tabular}


phase shift sensitivity among the best. But because for that group too the best singl frequency and the averaged sensitivities are less than using all variable-frequenc combinations, another variable is to be prefered.

Because with all of the approaches all controls scored normal, the specificity wit respect to the controls remains 1.00 , and so does not influence the choice of th sensitivity criterium.

The specifities regarding non-patients with respect to a specific diagnosis are given i table 9-10, using the standard reference ranges, and in table 9-11, using the unbiaset reference ranges. Also the sensitivities are given here. Using a smaller referenes range can be expected to result in higher sensitivities. This is found here for half o the group-frequency combinations, while the sensitivities for the other half of thi combinations remained the same. A higher sensitivity for one group depresses for al other group the specificity with respect to related classes. And because for the firs three stimulus frequencies several groups have increased sensitivities, naturally the specificities are here decreased for all combinations that include those frequencies Group 2 is the only one which has an increased sensitivity at $1.15 \mathrm{~Hz}$, and so for that group-frequency combination the specificity remains the same.

The poor sensitivities of the mtVVI phase shift when using the standard reference ranges make even the best combination of sensitivity and specificity unimpressive (sensitivity $=0.50$, specificity $=0.68$; for whiplash patients). Using the unbiased ranges, for two groups there is a best combination of sensitivity \& specificity: the Meniere patients $(0.75 \& 0.73)$ and those having BPPD $(0.67 \& 0.78)$.

\subsubsection{Individual contrasts of visuo-vestibular interaction with a visual target that is earth-fixed and with one that is moving asynchron- ously.}

In figures 9-7 and 9-8 the phase shift differences (mean \pm standard error) are depicted per group and per chair-delivered stimulus frequency. Conform the initial statistical analysis to be described below, the patient groups are divided in those who differed significantly from the controls, and those who did not, and outlying values are excluded. On average, the controls hardly differed between eftVVIT and mtVVIT (inferred from their phase shift differences being close to zero), while patients did. Using the same vertical scaling, figure $9-9$ gives the differences in phase shift responses to $\mathrm{mtVVI}$ stimulation and SP stimulation (same single-sine visual target movement, but without the sum-of-sine chair movement). Taking individual differences is intended to diminish group variability, and this has here some success. Compared to the ranges spanning approximately $10^{\circ}$ for the Meniere and the BPPD 


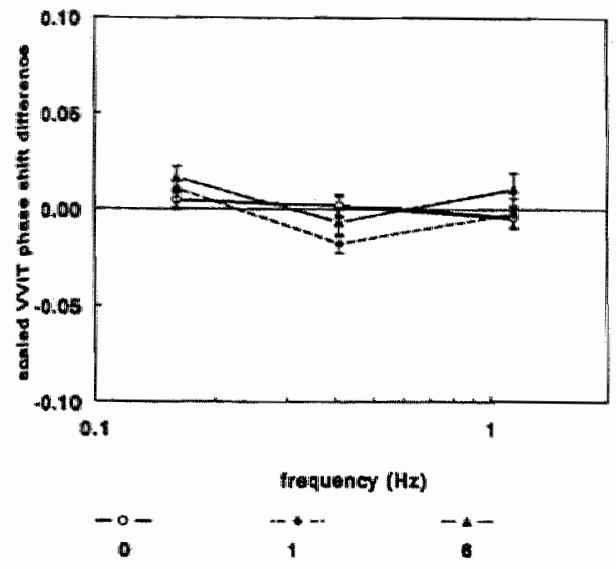

Fig. 19-7. Sealed phase shift difference depending on stimulus frequency for controls (O) and significantly different patients ( $*$ init. Meniere, $\Delta=$ BPPD).

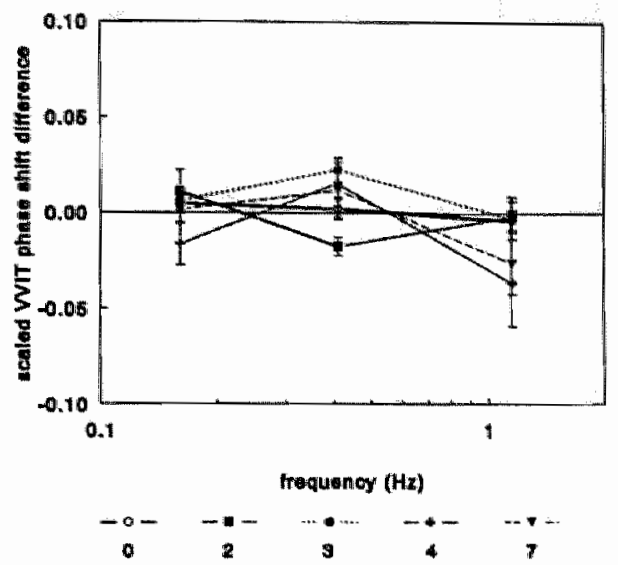

Fig. 9-8. Scaled phase shift differences depending on stim. freq for controls (o) and not significantly different pratients (I we mell comp. periph vest. dyst., - incompl. comap. periph. vest. dyst, $+=$ whîplash, * = unknown).

patients for SP stimulation and mtVVI stimulation (figures 9-3 and 9-6, respectively), the range of less than 0.02 (scaled difference, i.e., about $7^{\circ}$ ) is a reduction of $30 \%$. For other groups, however, the variability has increased.

The responses to the chair-delivered movements appeared to be non-Normal in Normal probability plots. Values for the parameter $\lambda$ for corrective Box-Cox transformations are given in table 9-12 (bold printing indicate combinations deviate severely). Their variation suggests that the observed deviations from Normality are not caused by inappropriate scaling and so individual outliers were sought. Using the above mentioned criteria resulted in detecting five outliers (table 9-13). These were excluded together with the results for the other frequencies of those subjects. The overall testing (table 9-14) estimated (5.0 $\pm 6.0) \times 10^{-3} \times$ age to be the effect of age on the phase difference, which is insignificant $(p=0.415)$, and neither was sex found to influence responses significantly $(\mathrm{p}=0.449$ for the interaction effect of sex and frequency, and $p=0.751$ for the remaining effect of sex). So both variables could be excluded from analysis

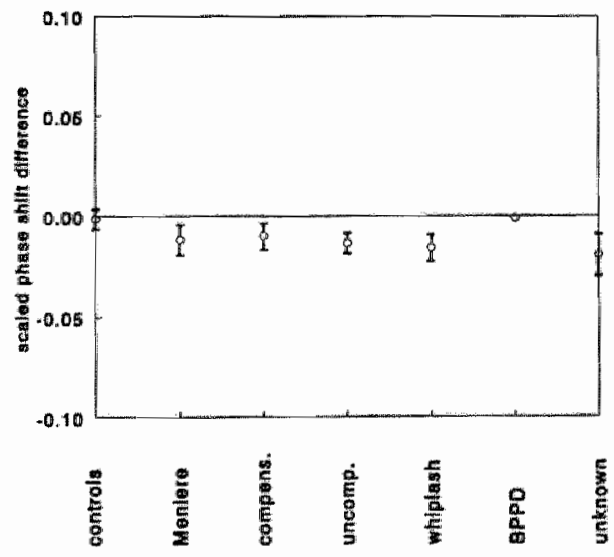

Fig 9-9. Phase shift response differences bethween singlesine $(0.53 \mathrm{~Hz})$ wisual target movement, simultanows and aymchronous with the summof sines chair mowement, and iderm without chair movement. Same vertical scaling as figure 9.7 and 9.8 


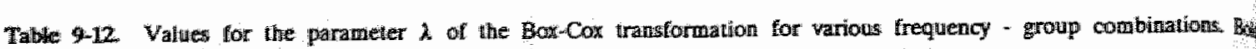
printed value indicate combinations that deviate 0,5 inits or more from the Normal distribution.

\begin{tabular}{|c|c|c|c|}
\hline Niagnosis: & $\begin{array}{l}0.16 \\
\mathrm{~Hz}\end{array}$ & $\begin{array}{l}0.41 \\
\mathrm{~Hz}\end{array}$ & $\begin{array}{l}1.15 \\
\mathrm{~Hz}\end{array}$ \\
\hline 0. cointrolis & 40 & 62 & 80 \\
\hline 1. inilial sitages of Metriere's aydirome & 28 & -34 & 15 \\
\hline 2. peripheral vestibular dystunction, centrally well compensated & 10 & -21 & 7 \\
\hline 3. peripheral vestibular dystumetion, centrally poorly compensated & 20 & 21 & -19 \\
\hline 4. whiplash tratuma & 1.2 & -9 & 8 \\
\hline 6. benign paroxysmal positioning dizumess & 270 & -95 & 57 \\
\hline 7. westibular complaints from unknown causes & 45 & -57 & 22 \\
\hline
\end{tabular}

safely. Repeating the overall MANOVA with diagnosis and frequency as the onl explaining variables, showed that groups differed significantly $(\mathrm{p}=0.052)$, and st pairs of groups may be tested.

The results of pairwise testing are shown in table 9-15.

Compared to the controls, groups 1 and 6 react in a significantly $(p=0.042$ and $p=$ 0.035 , respectively) different way to the stimulus frequencies, but do not differ in overall response. Groups 2, 3 and 4 do not distinct themselves from the controls. Fo: groups 7 and 12 there is insufficient evidence to conclude already that these patient scored differently from the controls. But neither may one conclude that they ar identical with the controls, given the small sample sizes and the rather low chanc probabilities ( $p=0.095$ and $p=0.071$, respectively).

In conclusion, two groups of patients (those in the initial stages of Meniere's syn

Table: 9-13. Ontlying response values for various frequency - group combinations. Criterion was deviatirg 0.5 units or mote fivo the Normal distribution as well as lying distinct from the bulk of data.

\begin{tabular}{|c|c|c|c|}
\hline diagnosis & $\begin{array}{l}0.16 \\
\mathrm{~Hz}\end{array}$ & $\begin{array}{l}0.4 \pi \\
\mathrm{Hz}\end{array}$ & $\begin{array}{l}1.15 \\
\mathrm{~Hz}\end{array}$ \\
\hline 0. conitrols & . & .. & $\ldots$ \\
\hline 1. initial stages of Meniere's symdrome &. & .. & -0.175 \\
\hline 2. peripheral vestibular dysfunction, centrally well compensated & $*$ & 0.1120 & .. \\
\hline 3. peripheral vestibulder dysfunction, cenurally poorly compensated. & 0.131 & wn & $*$ \\
\hline 4. Whiplasis trauma & $\ldots$ & 0.128 & -0.19 .8 \\
\hline 6. benign parnysmall positioning dizziness & . & $\ldots$ & $\ldots$ \\
\hline 7. vestibular complaints from unknown causes & 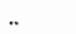 & .. & .*. \\
\hline
\end{tabular}


Table 9-14. Effect of excluding independent variables from multivariate analysis of the VVTT phase difference walue. Within subjects factor. frequency, between subjects factors; sex, diggnosis; cowariate age. Stars indicate inuportant group differences.

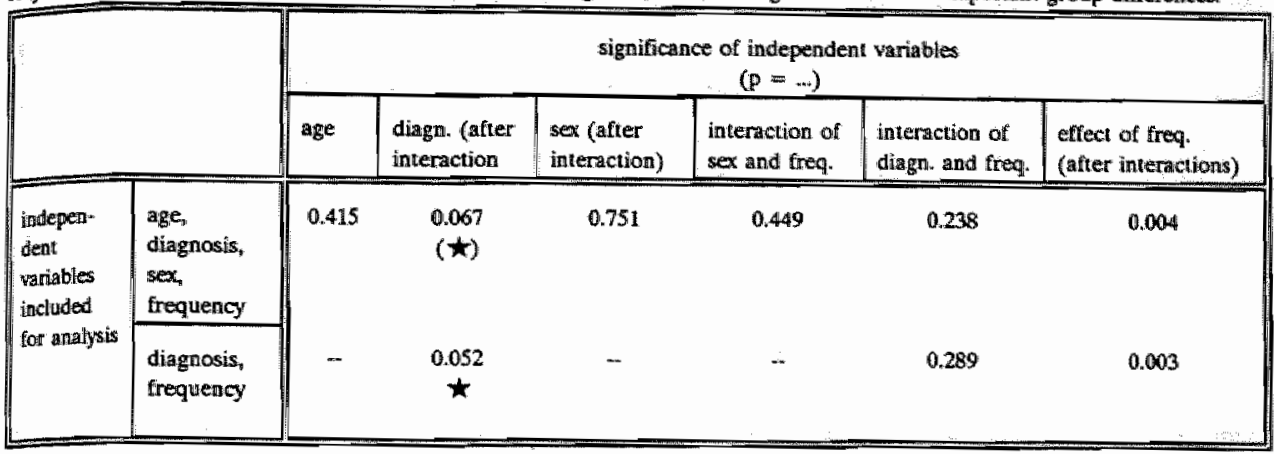

(丈) suggestive, but not significant at $5 \%$ level

$\star \quad$ significant at $5 \%$ lewel

drome and those with benign paroxysmal positioning vertigo) produce deviant VVTT phase shift differences, two groups (those with unknown causes of their vertigo complaints and those with multiple vestibular dysfunctioning) might do so and three groups (those with a peripheral vestibular dysfunctioning, either or not centrally compensated, and those with whiplash trauma) produce normal VVIT phase shift differences.

The clinical usefulness is assessed by calculating the sensitivities and specificities for the various diagnostic groups. Table 9-16 shows that using the unbiased reference

Tabite 9-15. MANOWA results of painvise comparison of VWT pluase sbift differences from various patient groups whth a control group $(n=6)$, with diagnosis and stimulus frequency as independent wariables. Stars indicate imaportant group differences.

\begin{tabular}{|c|c|c|c|c|}
\hline \multirow[t]{2}{*}{ diagnosis } & \multirow{2}{*}{$\begin{array}{l}\text { number } \\
\text { of } \\
\text { nembers }\end{array}$} & \multicolumn{3}{|c|}{$\begin{array}{c}\text { signifietance } \\
(p=.)\end{array}$} \\
\hline & & $\begin{array}{l}\text { diagnosist } \\
\text { (after } \\
\text { intheraction) }\end{array}$ & $\begin{array}{l}\text { "interaction of } \\
\text { diagnosis and } \\
\text { frequency }\end{array}$ & $\begin{array}{l}\text { Irequency } \\
\text { (after } \\
\text { interaction) }\end{array}$ \\
\hline 1. initial stages of Meniere's syndrome & 3 & 0.620 & $0.042 \hbar$ & 0.043 \\
\hline 2. peripheral vestibular dysfunction ${ }_{4}$ well compensated & 7 & 0.165 & 0.500 & 0.090 \\
\hline 3. peripheral westibular dysfunction, poorly compensated & 12 & 0.544 & 0.401 & 0.118 \\
\hline 4. Whiplash trauma & 8 & 0.1.58 & 0.3514 & 0.055 \\
\hline 6. benign paroxymmal positioning dixzśness & 3 & 0.275 & $0.035 \star$ & 0,083 \\
\hline 7. vestibutiar complaints from unknown causes & 4 & 0.213 & $0.095(x)$ & 0.000 \\
\hline
\end{tabular}




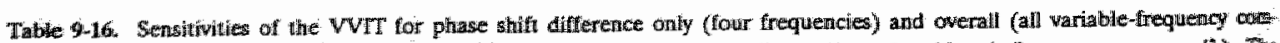

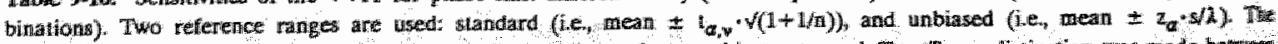

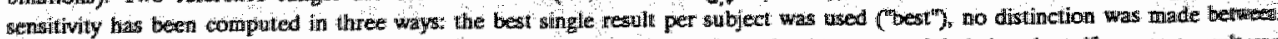

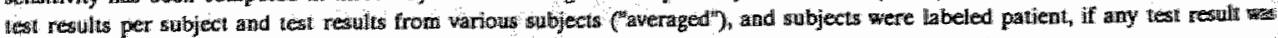

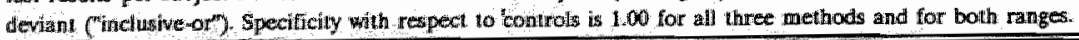

\begin{tabular}{|c|c|c|c|c|c|c|c|c|c|c|c|c|}
\hline \multirow[t]{2}{*}{ diagnosits } & \multicolumn{6}{|c|}{ phase shiff difference bensitiwity } & \multicolumn{6}{|c|}{ overall sensitivity } \\
\hline & best & $\begin{array}{l}\text { aver } \\
\text { agsol }\end{array}$ & $\begin{array}{l}\text { inct. } \\
\text { or }\end{array}$ & best & $\begin{array}{l}\text { awer- } \\
\text { aged }\end{array}$ & incl. & besil & $\begin{array}{l}\text { awer- } \\
\text { agent }\end{array}$ & $\begin{array}{l}\text { incl. } \\
\text { or: }\end{array}$ & best & $\begin{array}{l}\text { aver- } \\
\text { aged }\end{array}$ & $\begin{array}{l}\text { incl. } \\
\text { or: }\end{array}$ \\
\hline 1. Minivire & 0.50 & 0.25 & 0.75 & 0.50 & 0.31 & 0,75 & 5.00 & 0.51 & 1.00 & 1.00 & 0.66 & 1.00 \\
\hline 2. compensalted & 0.38 & 0.31 & 0.63 & 0.50 & 0.34 & 0.63 & 0.75 & 0.34 & 0.88 & 0.75 & 0.42 & 0.8 \\
\hline 3. unicompensated & 0.23 & 0.17 & 0.38 & 0.46 & 0.29 & 0.62 & 0.92 & 0.35 & 1.00 & 0.92 & 0.42 & 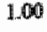 \\
\hline 4. Whiptash & 0.60 & 0.49 & 0.80 & 0,60 & 0.48 & 0.80 & 1.00 & 0.51 & 1.00 & 1.00 & 0.60 & 40 \\
\hline 6. $\mathrm{BPPD}$ & 0,00 & 0.000 & 0.00 & 0.33 & 0.33 & 0.33 & 1.00 & 0.42 & 1.00 & 1.00 & 0.52 & 1,00 \\
\hline \multirow[t]{2}{*}{ 7. unkntiown } & 0.25 & 0.19 & 0.50 & 0.50 & 0.31 & 0.50 & 0.50 & 0.30 & 0.50 & 0.75 & 0.36 & 100 \\
\hline & \multicolumn{3}{|c|}{$\begin{array}{l}\text { standard } \\
\text { reference range }\end{array}$} & \multicolumn{3}{|c|}{$\begin{array}{l}\text { unibiased } \\
\text { reference range }\end{array}$} & \multicolumn{3}{|c|}{$\begin{array}{l}\text { standard } \\
\text { reference range }\end{array}$} & \multicolumn{3}{|c|}{$\begin{array}{l}\text { unbiased } \\
\text { reference range }\end{array}$} \\
\hline
\end{tabular}

ranges results in higher sensitivities than when using the standard reference ranges. Comparing the phase shift difference sensitivities with those calculated using all variable-frequency combinations, reveals that the first are worse than on average. Because with all of the approaches al] controls scored normal, the specificity with respect to the controls remains 1.00 , and so does not influence the choice of the

Table 9-17 Sensitiviry and specificity (neganding non-patients with respect to specific disgnosis) per diagacustic group and per frequency, using standard reference ranges. Mimimall value of squared error sum (indicated by gray cell) gives optinul combination.

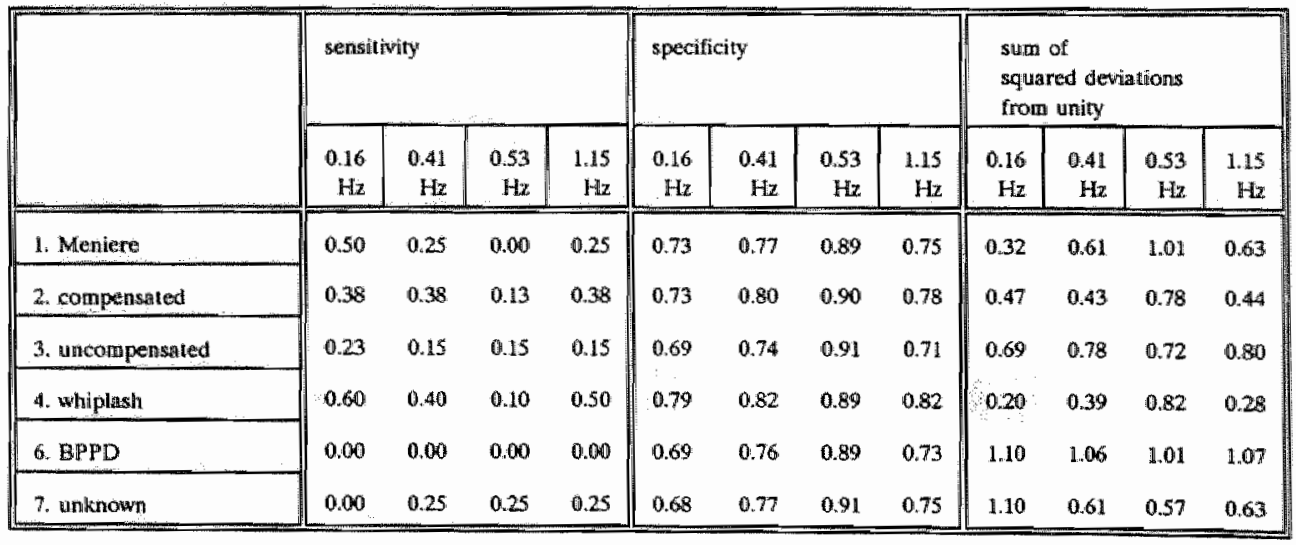




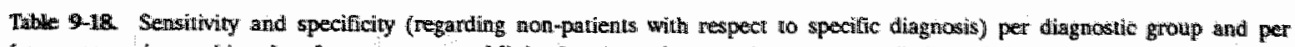
tazuency, using unbiased reference ranges. Minimal value of squared error sum (indicated by gray celly gives optimall combunkationt.

\begin{tabular}{|c|c|c|c|c|c|c|c|c|c|c|c|c|}
\hline & \multicolumn{4}{|c|}{ sensitivity } & \multicolumn{4}{|c|}{ specificity } & \multicolumn{4}{|c|}{$\begin{array}{l}\text { sum of } \\
\text { squared devitutions } \\
\text { from thity }\end{array}$} \\
\hline & $\begin{array}{r}0.16 \\
\mathrm{~Hz} \\
\end{array}$ & $\begin{array}{r}0.41 \\
\mathrm{~Hz} \\
\end{array}$ & $\begin{array}{r}0.53 \\
\mathrm{H} i 2 \\
\end{array}$ & $\begin{array}{r}1.15 \\
\mathrm{~Hz}\end{array}$ & $\begin{array}{r}0.16 \\
\mathrm{~Hz} \\
\end{array}$ & $\begin{array}{r}0.41 \\
\mathrm{~Hz} \\
\end{array}$ & $\begin{array}{r}0.53 \\
\mathrm{H} i \\
\end{array}$ & $\begin{array}{r}1.15 \\
\mathrm{~Hz} \\
\end{array}$ & $\begin{array}{r}0.16 \\
\mathrm{~Hz} \\
\end{array}$ & $\begin{array}{r}0.41 \\
\mathrm{Hiz} \\
\end{array}$ & $\begin{array}{r}0.53 \\
\mathrm{Hiz}\end{array}$ & $\begin{array}{r}1.15 \\
\mathrm{~Hz}\end{array}$ \\
\hline 1. Meniere & 0.50 & 0.50 & 0.00 & 0.25 & 0.68 & 0.73 & 0.82 & 0.61 & 0.35 & 0.32 & 1.03 & 0.71 \\
\hline 2. compensated & 0.38 & 0.50 & 0.13 & 0.38 & 0.68 & 0.75 & 0.83 & 0.63 & 0.50 & 0.31 & 0.80 & 0.53 \\
\hline 3. uncompensated & 0.31 & 0.15 & 0.23 & 0.46 & 0.66 & 0.66 & 0.86 & 0.66 & 0.60 & 0.83 & 0,61 & 0.41 \\
\hline 4. whiplashi & 0,60 & 0.50 & 0.20 & 0.60 & 074 & 0.76 & 0.84 & 0.68 & 0,23 & 0.31 & 0.66 & 0.26 \\
\hline 6. BPPD & 0.00 & 0.00 & 0.00 & 0.33 & 0.64 & 0.69 & 0.82 & 0.62 & 1.13 & 2.10 & 1.03 & 0.59 \\
\hline 7. unkmown & 0.25 & 0.25 & 0.50 & 0.25 & 0.66 & 0.70 & 0.86 & 0.61 & 0.68 & 0.65 & 0.27 & 0.71 \\
\hline
\end{tabular}

sensitivity criterium.

The specifities regarding non-patients with respect to a specific diagnosis are given in table 9-17, using the standard reference ranges, and in table 9-18, using the unbiased reference ranges. Also the sensitivities are given here. Using a smaller reference range can be expected to result in higher sensitivities. This is found here for nearly half of the group-frequency combinations, while the sensitivities for the other combinations remained the same. A higher sensitivity for one group depresses for an other group the specificity with respect to related classes. And because at each stimulus frequency there are at least two groups with an increased sensitivity, the specificities of all group-frequency combinations naturally are less when using the unbiased reference ranges.

The conclusions are not greatly affected by this change in reference range width: the group having the best combination of sensitivity and specificity is formed by the whiplash patients (sensitivity $=0.60$, specificity $\approx 0.76$ ), while the Meniere patients keep the same values (sensitivity $=0.50$, specificity $=0.73$ ). Due to their small group size, fluctuations in the scores of the patients with unknown causes for their vestibular complaints have drastic effects.

\subsubsection{Conclusions.}

The results of the MANOVA and of the sensitivity and specificity analysis and for 


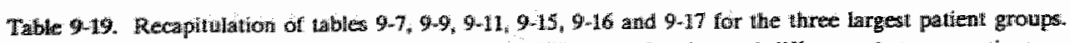

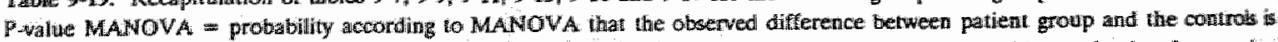

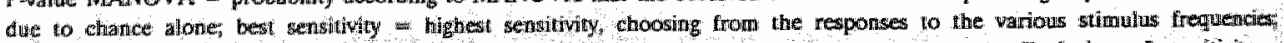

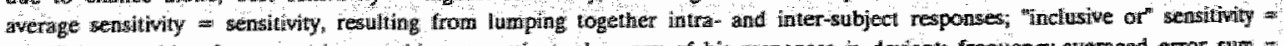

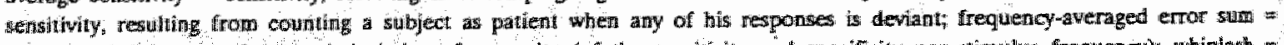

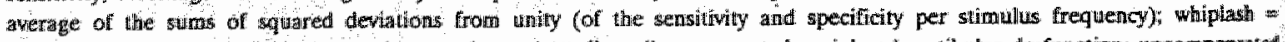
whiphas patiens" compensated = patierts having a centrally well compensated peripheral westibular dystrunction; uncompensater - panients hwing a contrally poorly conpensited peripheral vestibular dystunction.

\begin{tabular}{|c|c|c|c|c|c|c|c|c|c|c|}
\hline & \multicolumn{5}{|c|}{ trVVI phase shift sensilivity } & \multicolumn{5}{|c|}{ VI phase shifi difference } \\
\hline & whiplash & $\mathrm{cot}$ & pent- & & mpen- & Whiplass & $\begin{array}{l}\mathrm{co} \\
\mathrm{sat}\end{array}$ & $\begin{array}{l}\text { pen- } \\
\text { d }\end{array}$ & & $\begin{array}{l}\text { unper:- } \\
1\end{array}$ \\
\hline pualue MANOVA & 0.037 & $<$ & 0.054 & $<$ & 0.723 & 0.158 & $<$ & 0.165 & $<$ & 0.401 \\
\hline best sensiturity & 0.50 & 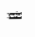 & 0.50 & $>$ & 0.31 & 0.60 & $>$ & 0.50 & $>$ & 0.46 \\
\hline avenge sensitivity & 9.28 & $\approx$ & 0,28 & $>$ & 0.17 & 0.48 & $>$ & 0.34 & $>$ & 0.29 \\
\hline melusive or sensitivity & 0.80 & $s$ & 0.63 & $>$ & 0.54 & 0.80 & $>$ & 0.63 & $>$ & 0.62 \\
\hline frequency-averaged error sum. & 0.50 & $=$ & 0.50 & $<$ & 0.78 & 0.42 & $<$ & 0.53 & $\ll$ & 0.75 \\
\hline
\end{tabular}

miVVI phase shift and for phase shift difference all seem to disagree. This confusion is caused by the small sample sizes. Especially for the sensitivity and specificity analysis, a small change in response can result in a dramatically different outcome (e.g., one extra score for the Meniere patients lifts the sensitivity from 0.25 to 0.50 ). We therefore concentrate on the three largest groups: patients having a centrally poorly compensated peripheral vestibular dysfunction (13), whiplash victims (10), and patients having a centrally well compensated peripheral vestibular dysfunction (8). Then all analyses find the same order: whiplash, compensated, uncompensated (decreasing deviation from normals; table 9-19).

For the patients having peripheral vestibular dysfunctions, the deviant phase shifts seems to be an adaptation. This corresponds with the mechanism of adaptation as given by Baloh et al. (1984): at intermediate frequencies is the gain proportional to the sensitivity constant divided by the dominant time constant, so that a lesser sensitivity can be compensated by an increased phase shift. Whether the deviant phase shifts of the whiplash victims are also an adaption, or whether it is an impaired function in itself, remains unsolved. Comparing new and old whiplash victims should clarify this. Given the normal gains for slow harmonic oscillation for most whiplash patients, it seems less likely that the different phase shifts reflect an adaptation to a decreased sensitivity. 


\section{Effect of asynchronous visual stimulation on the Vestibulo-Ocular Reflex coherence of patients, compared to that of normals. 10.1 Abstract.}

A vestibular function test (named Visuo-Vestibular Interaction Test: VVIT) is presented, in which a visual target may move asynchronoushy with a rotatory chair. The coherence responses and their individual differences for two modes of stimulation ( 1 sum-of-sines chair movement with an earth-fixed visual target, 2 sum-of-sines chair movement with an independent sinusoidal target movement) are statistically analyzed for six groups of different types of vestibular patients (totalling 42 subjects) and a control group ( 6 subjects). Patients were diagnosed using anamneses and standard tests. Here, only the coherences are considered, the results of analyzing the gains and the phase shifts are reported elsewhere (chapters 8 and 9 , respectively).

In descending order of deviation, patients having benign paroxysmal positioning dizziness (BPPD), whiplash patients, Meniere patients, centrally poorly compensated peripheral vestibular patients, and centrally well compensated peripheral vestibular patients differ significantly $(\mathrm{p}<0.025)$ from normals, while patients with vestibular complaints from unknown origin differ suggestively, but not significantly $(p=0.067)$.

Using reference ranges based on normals only, the coherence when vestibulary stimulated in presence of a moving target, is the most sensitive variable. The relative difference of the coherences resulting from being stimulated in the two abovementioned modes, in contrast, allows for the three lower stimulus frequencies distinction between Meniere patients, whiplash patients, and BPPD patients at the one hand, and peripheral vestibular patients (both compensated and uncompensated), patients with complaints from unknown origin, and controls at the other hand.

\subsection{Introduction.}

A full introduction of the VVIT was given earlier (chapter 5: "Effect of asynchronous visual stimulation on the Vestibulo-Ocular Reflex gain of normals"), so here only the essentials will be repeated.

In order to elude the variation inherent to measuring the vestibulo-ocular reflex (VOR) in the dark, a visual target is presented. The usual ways in which the resulting visuo-vestibular interaction (VVI) is studied, (namely, by offering either an earth-fixed target or a chair-fixed target) allow, but may not require, the two systems to interact. To force an interaction between smooth pursuit (SP) and VOR, we use a visual target which moves asynchronously with the chair. Predictive effects are reduced by having the chair move in a pseudo random (sum-of-sines) way. As both visual target 
movement and chair movement are below the SP upper limit, we expect normals and patients to perform equally well to either one of them. But because the combination is above the SP upper limit, we expect patients to perform worse than normals to the combined stimulus, assuming that they will overburden their SP system in an effort to compensate for the insufficient vestibular function. By subtracting the visuo-vestibular interaction with an earth-fixed target (eftVI; ie., visually enhanced VOR) stimulus and the response to it, from the visuo-vestibular interaction with an asynchronously moving target (mtVVI) stimulus and the response to it, the visual components are obtained. It is hypothesized that the vestibular function is reflected by the ratio of the smooth and saccadic eye movements (and thus by the gain of the median-filtered response). It is also expected that the patients' coherence will detoriate, compared to that of the normals.

\subsection{Material and methods. 10.3.1 Subjects.}

Data were obtained from the subjects who were described earlier (see chapter 8 ). In short, 42 patients (median age: 45) and 6 controls (median age: 30 ) were examined. The patients felt in 6 categories: those having initial stages of Meniere's syndrome (4), those having peripheral vestibular dysfunctions, centrally well compensated (8) and centrally partly compensated (13), whiplash victims (10), BPPD patients (3), and those for which the causes of their complaints are unknown (4). They were diagnosed by an experienced vestibulologist (dr. Kingma), using anamneses and results of standard tests (see below). All subjects were tested without optical corrections.

\subsubsection{Experimental set-up.}

The experimental set-up was as teported previously (chapter 5), and so only a brief description follows here. Subjects sat in a rotary chair moving in a pseudo random way (sum-of-sines: $0.16,0.41$, and $1.15 \mathrm{~Hz}$, each with a maximum velocity of $10 \% / \mathrm{s}$ ), while watching a dot which was either earth-fixed or moving independently $(0.53 \mathrm{~Hz}$, with a maximum velocity of $32 \% / \mathrm{s}$ ). The eye position was measured using infra red reflection, and the head velocity was monitored with an angular rate sensor. Eye position, head velocity, and visual target position were sampled at a $50 \mathrm{~Hz}$ frequency for 125 seconds by a 12 bit analog-to-digital (AD) converter (the first two after being filtered analogously with a cut-off frequency of $10 \mathrm{~Hz}$ ) and were digitally stored for 
offline analysis.

Standard tests consisted of calorization ( $30^{\circ}$ Celsius and $44^{\circ}$ Celsius, in the order right cold, left cold, left warm, right warm), torsion in the dark $(0.1 \mathrm{~Hz}$ frequency, 50 - /s peak velocity), a smooth pursuit test $\left(0.29 \mathrm{~Hz}\right.$ frequency, $15^{\circ}$ amplitude), and an optokinetic test $(10,20,30$, and $40 \% / s$ velocity, both horair and anti-horair). Left and right horizontal eye position was measured by electro-oculography, and was sampled at $100 \mathrm{~Hz}$.

\subsubsection{Digital signal analysis.}

Processing and analysis of the signals was as reported before (see chapter 5). In summary, position data were digitally differentiated to velocities, after which the total stimulus was combined from visual target movement and head movement. After median filtering and Welch windowing, half-overlapping segments of the data were Fourier transformed, and the power spectra derived from these, were averaged. Further frequency-averaging was done, until the product of segment-averaging and frequency-averaging equaled 40 (Bendat \& Piersol, 1971, p 191-194). The coherence function (Bendat \& Piersol, 1971, p 337-339) was calculated, and finally those values at the response frequencies were extracted automatically from the spectrum using preset criteria. The individual contrasts of the eftVVI and the mtVVI measurements were calculated as relative differences, and were also analyzed statistically.

\subsubsection{Statistical analysis.}

Statistical analysis was as reported earlier (see chapter 8). After removing univariate non-Normal outliers, a multivariate analysis of variance (MANOVA) was done to assess the significances with which various factors correlated with the observed differences of the means. Four influences were analyzed: the within-subject factor stimulus frequency, the between-subjects factors sex and diagnosis, and the covariate age. The distribution of the residuals was used to detect multivariate outliers.

Sensitivity was calculated per diagnostic group for two reference ranges (a standard one (Albert \& Harris, 1987) and an unbiased one (Royston \& Matthews, 1991); see chapter 5 ) in three ways ( $\underline{1}$ using each subject's best single result, 2 lumping together intra-subject and inter-subject results, $\underline{3}$ labelling subjects as patients if any of their 
intra-subject results was outside the reference ranges). Specificity was calculated regarding controls only, as well as regarding other diagnostic groups and controls.

\subsection{Results and discussion.}

The VVIT consists of chair movements with a visual target that is earth-fixed, and with one that moves asynchronously. And so three sets of data are obtained: one resulting from the first stimulus condition (eftVVI), one resulting from the second stimulus condition (mtVVI), and one resulting from taking the individual differences of the first two sets (contrast; calculating the means of the individual differences gives the same values as taking the differences of the means, but produces different variances).

For all three sets, the distributions of the responses were checked on Normality by means of Normal probability plots. In case of deviations, two strategies are possible for Normalizing the data. If one considers all data equally important, then transforming the data can be tried. Alternatively, some data can be considered outliers and can be excluded.

To see whether the first strategy was feasible, values for the parameter $\lambda$ for corrective Box-Cox transformations (see the appendix of chapter 8) were calculated for each frequency-group combination. The more combinations need similar transformations, the more probable it is that the observed deviations from Normality are caused by inappropriate scaling. If no single transformation will do for all combinations, then the second strategy has to be used. If in a Normal probability plot a value deviated 0.5 units or more from the Normal distribution and was lying distinct from the bulk of data, it was considered to be an outlier, eligible for removal. The responses of that same subject to the other stimulus frequencies were then also removed, irrespective whether they fitted in the Normal distribution.

The Normal probability plots strongly suggest that diagnostically defined group 3 consists of two differently reacting subgroups. This is studied in more detail in part 1 "Group 3 might consist of two sub groups" of chapter 13 "General discussion". Because the distinction is suggested by the current VVIT data only, and not by anamneses or standard test outcomes, it would be inappropriate to split the group and statistically test the subgroups.

To assess the clinical usefulness of a test, its sensitivity (i.e., the ratio of found and actual patients) and its specificity (i.e., the ratio of found and actual non-patients) are calculated (e.g., Sacket et al., 1985, p 69-72). This is straightforward in case of a 
univariate test and one type of patients, but becomes a little more complicated in our case. The current VVIT produces 26 results $^{3}$, and in the current study the results of six types of patients ${ }^{2}$ are analyzed. Of course, all the data generated by the VVIT should be used to determine whether a subject is a patient, and if so, what type of patient he is. This can be done by combining the results with weights as found by cluster analysis (see chapter 11 "Discriminating power of VVIT for type of pathology, compared to those of standard tests"). With these end results the sensitivity and specificity can be calculated in the usual way. Nevertheless, it is also interesting to see whether the univariate results alone would allow us to draw valid conclusions, as that would greatly simplify diagnosis. Having various results, one may give the best sensitivity for a single variable-frequency combination (which can be different combinations for various groups of patients), the average sensitivity (i.e., all positive test outcomes, divided by the product of the number of cutcomes per test times the number of patients), or the "inclusive-or" sensitivity (i.e., the sensitivity when a subject is considered to be a patient if any of the test results deviates significantly from those of the normals). And one may give the specificity regarding controls only (i.e., no patients for any type of vestibular dysfunction), or for non-patients with respect to a specific type of vestibular dysfunction (and thus including other vestibular patients as well as controls).

\subsubsection{Visuo-vestibular interaction with an earth-fixed visual target.}

In figures 10-1 and 10-2 the coherence values (mean \pm standard error) for visuovestibular interaction with an earth-fixed target (eftVVI) are depicted per group and per chair-delivered stimulus frequency. The patient groups are distributed over two figures for clarity only, none differed significantly from the controls (see below). Conform the statistical analysis to be described below, outlying values are excluded. Figure 10-3 shows the coherence values per diagnostic group for the SP stimulus $(0.53 \mathrm{~Hz})$. All patient groups performed worse than the control group, but due to their variations, those differences are statistically insignificant.

The responses appeared to be non-Normal in Normal probability plots. Values for the parameter $\lambda$ for corrective Box-Cox transformations are given in table 10-1 (bold

\footnotetext{
126 VVIT results: eftVVI phase shift and eff VVl collerence, both for three frequencies, and gain contrasit, miVVI phase shiff, phase shift difference, miVl coherence, and coherence contrast, each for four frequencies.

${ }^{2}$ Six types of patients: initial stages of Meniere's disease, centrally well compenisated peripheral vestibular dysfunction, centrally poorly compensated peripheral vestibular dysfunction, whiplash trauma, benign paroxysmal positioning dizziness, vestibular complaimts trom unkmown origin.
} 


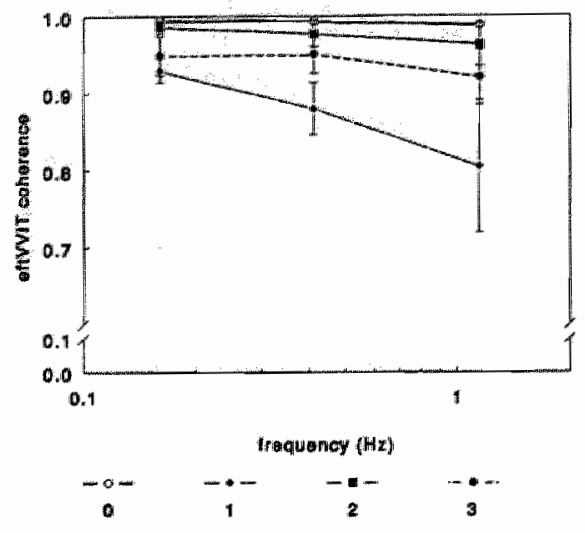

Fig. 10-1. efVVIT coherence depending on stimulus frequency for controls (o) and not significantly different patients. ( $=$ init Meniere, $=$ well comp periph. west. dyst, $=$ incompl. comp. periph, vest. dyst.).

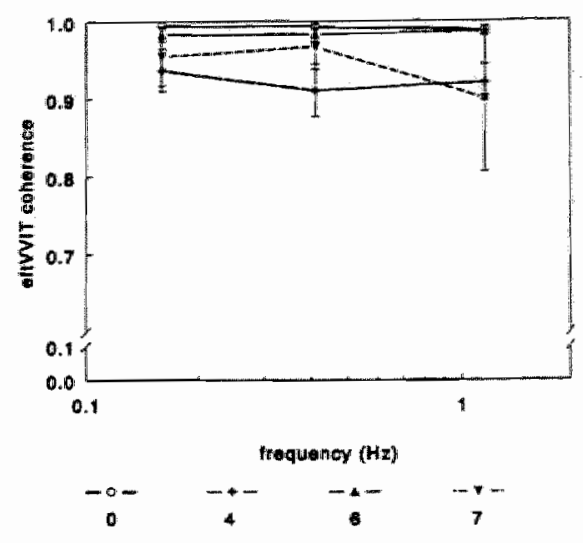

Fig 10-2 efrVTT coherence depending on stimults frequency for controls $(0)$ and not significantly differezt: patients $\left(+=\right.$ whiplash $_{2} *=$ benign paroxysm. posit. vertigo, $=$ unknowim).

printing indicate combinations deviate severely). Their variation suggests that the observed deviations from Normality are not caused by inappropriate scaling, and so individual outliers were sought. Using the above mentioned criteria, resulted in detecting three outliers (table 10-2), all belonging to the same subject. These data were excluded and subsequent analysis was done on the remaining set.

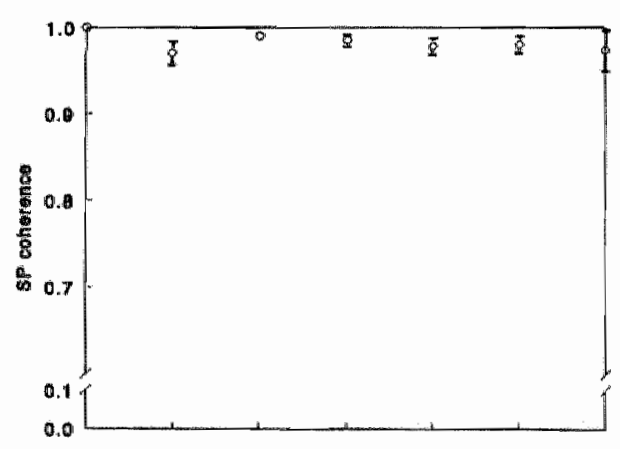

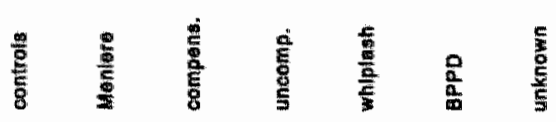

The overall testing (table 10-3) estimated $(-2.8 \pm 3.5) \times 10^{-2} \times$ age to be the effect of age on the eftVVIT coherence, which is insignificant $(p=0.431)$.

Because overall testing failed to show any significant (or even any suggestive) difference between groups, no pairwise testing is allowed.

Assuming that not finding any significant difference in group means implies that individual values will be rather similar for patients and controls, they are not compared to a reference range.

Fig. 10-3. Coherence values resulting from single-sine 0.53 Hz) SP. stimulation, of the various groups. Same vertical scaling as tigures 10-1 and $10-2$. 
Thible 10-1. Values for the parameter $\lambda$ of the Box-Cox transformation for warions frequency - igroup wombinations. Bold prituled values indicate combinatuions thiat deviste 0.5 units or more from the Nomal distribation.

\begin{tabular}{|c|c|c|c|}
\hline ditagnosilis & $\begin{array}{l}0.16 \\
\mathrm{~Hz}\end{array}$ & $\begin{array}{l}0.1 \\
H x\end{array}$ & $\begin{array}{l}1.15 \\
\mathrm{~Hz}\end{array}$ \\
\hline a. conatiols & 0 & 113 & 60 \\
\hline 1. initial stages of Meniere"s syndrome & -10 & 13 & 3.6 \\
\hline 2. peripheral vestibular dysfunetion centrally well compensated & 52 & 39 & 22 \\
\hline 3. peripheral vestibular dystunction, centrally poorly compensated & 15 & 16 & 11 \\
\hline 4. Whipulash tratumia & 11 & 6.7 & 4.5 \\
\hline 6. benign parcoxysmai positioning dizziness & .26 & .26 & -106 \\
\hline 7 westibular complaints from unknown causes. & 24 & 26 & 71 \\
\hline
\end{tabular}

Table 10-2 Outlying response values for various frequency - group combinations. Criterion was deviating 0.5 umits or more from the Normal distribution as well as lying distanct from the bulk of data.

\begin{tabular}{|c|c|c|c|}
\hline diagnosis & $\begin{array}{l}0.16 \\
\mathrm{~Hz}\end{array}$ & $\begin{array}{l}0.41 \\
\mathrm{H} x\end{array}$ & $\begin{array}{l}1.15 \\
\mathrm{H} x\end{array}$ \\
\hline 1. contrais & $\cdots$ & .. & .. \\
\hline 1. initial stages of Meniere's syndrome & .* & $n \cdot$ & . \\
\hline 2. peripherall vestibular dysfurction, centrally well compensated & 0.93 & 0.86 & 0.77 \\
\hline 3. peripheral vestibular dysfiunction, centrally poorly compensated & * & ." & $m$ \\
\hline 4. whiplash trauma & $\cdots$ & $\cdots$ & * \\
\hline 6. benigm paroxysmal positioning dizziness & " & $*$ & $*$ \\
\hline 7. vestubutar tomplants witll unknom canses & $\therefore$ & $\therefore$ & a+ \\
\hline
\end{tabular}

Table 10-3. Onverall multivariate analysis of variance of the efrVIT coherence walues with and withouit age and sex as explaining warialbles. Within-subjectsi factor frequency, between-subjects factors. sex, diagnotis; covariate: age.

\begin{tabular}{|c|c|c|c|c|c|c|c|}
\hline & & \multicolumn{6}{|c|}{$\begin{array}{l}\text { significance of independent variable } \\
\qquad(\mathrm{p}, \ldots)\end{array}$} \\
\hline & & arge & $\begin{array}{l}\text { dilagn. (after } \\
\text { interaction) }\end{array}$ & $\begin{array}{l}\text { sex (after } \\
\text { untertaction) }\end{array}$ & $\begin{array}{l}\text { interaction } \\
\text { of sex and } \\
\text { freg. }\end{array}$ & $\begin{array}{l}\text { interaction: } \\
\text { of dliagn. } \\
\text { and frecj. }\end{array}$ & $\begin{array}{l}\text { wfiect of } \\
\text { freq. (afver } \\
\text { Hnteractions) }\end{array}$ \\
\hline \multirow{2}{*}{$\begin{array}{l}\text { indepen- } \\
\text { dent } \\
\text { wariables } \\
\text { nicluded } \\
\text { ror analysis }\end{array}$} & $\begin{array}{l}\text { age; diagnosis, } \\
\text { sex; }_{\text {f }} \text { frequency }\end{array}$ & 0.431 & 0.363 & 0.431 & 0.422 & 10.345 & 0.048 \\
\hline & $\begin{array}{l}\text { diagnosis } \\
\text { frequency }\end{array}$ & - & 0.298 & - & - & 0.232 & 0.298 \\
\hline
\end{tabular}




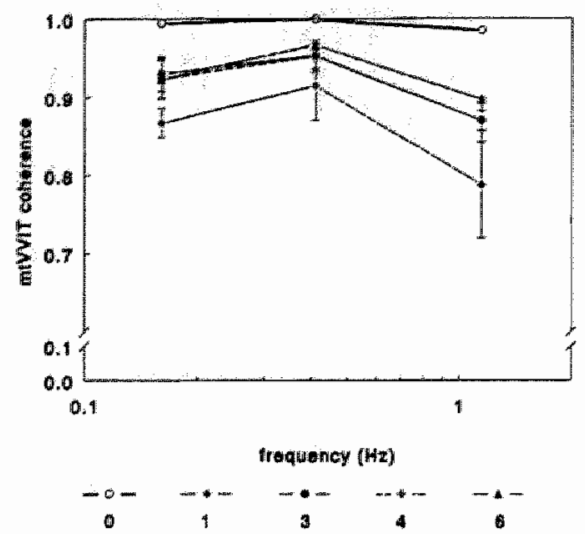

Fig 10-4. mitVIT colsenence depending on stimulus frequency for controls ( $O$ ) and significantly different patients (* $+=$ whiplasth, $=$ benign paroxysm. posit. vertigoy.

\subsubsection{Visuo-vestibular interaction with an asynchronously movir visual target.}

In figures 10-4 and 10-5 the coherence values (mean \pm standard error) for visu vestibular interaction with a moving target (mtVVI) are depicted per group and $p$ chair-delivered stimulus frequency. Conform the statistical analysis to be describi

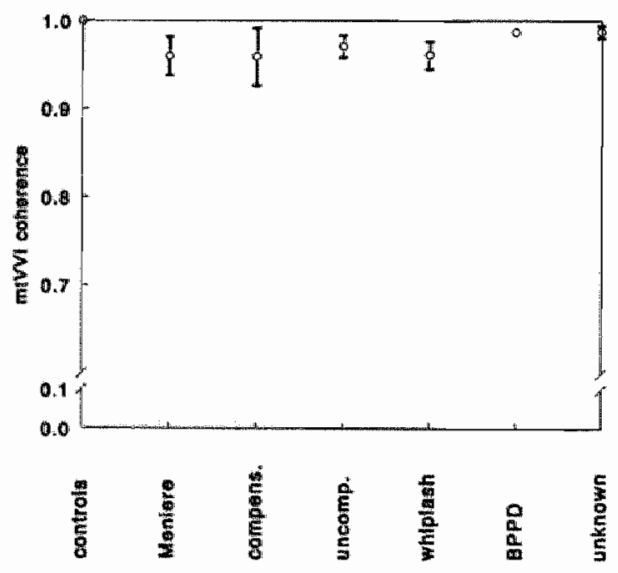

Fig. 10-6. Coherence waltues resulting from single-sine $\{0.53$ Hz) visual target mowement, simultanous and asynchronous with the sum-ol-simes chair movement. Same vertical scaling as figure $10-4$ and $10-5$.

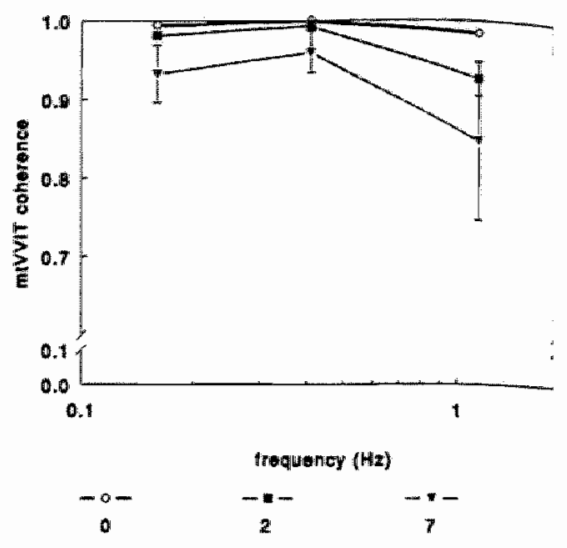

Fis 10-5. mivWII coherence depending on stimu frequency for controls (o) and not sigrificanilyy tiffer patients $(*=$ well comp. unilat. periph. west. Hyst., unknown). below, the patient groups are divided those who differed significantly from $t$ controls, and those who did not, and or lying values are excluded. Figure 10 shows the coherence values per diagnos group for the purely visually delivered $p$ of the mtVVI stimulus. All patient grou performed worse than the control grou although due to their variations, for son this was not statistically significant. groups performed better at $0.41 \mathrm{~Hz}$ th at the other chair frequencies. Because had their best response at $0.53 \mathrm{~Hz}$ (mc values in figure 10-6 are closer to ze than those in figures $10-4$ and 10-5), whi was delivered by the movement of $t$ visual target, and because frequency bi 


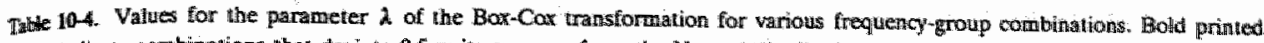
ald indicate combinations that deviate 0.5 units or more from the Nomat distribution.

\begin{tabular}{|c|c|c|c|}
\hline diagnosis & $\begin{array}{l}0.16 \\
\mathrm{~Hz}\end{array}$ & 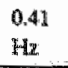 & $\begin{array}{l}1.15 \\
\mathrm{~Hz}\end{array}$ \\
\hline 0. comitrols & .214 & - & 3.0 \\
\hline I. initial stages of Meniere's syndrome & 32 & 73 & 1.0 \\
\hline 2. peripheral vestibular dysfunction, centrally well compensated & 22 & 15 & 0.27 \\
\hline 3. peripheral westibular dystunetion, centrally poorly compensated & 12 & 13 & 5.5 \\
\hline 4. shipiash tranma & 46 & 7.6 & 6.0 \\
\hline 6. benign paroxysmal positioning dizziness & 114 & 208 & 160 \\
\hline 7. vestöbular complaints from unkinown causes & 17 & 106 & 5.4 \\
\hline
\end{tabular}

were averaged prior to calculating the coherence, the high $0.41 \mathrm{~Hz}$ response might be an artefact of analysis.

The responses appeared to be non-Normal in Normal probability plots. Values for the parameter $\lambda$ for corrective Box-Cox transformations are given in table 10-4 (bold printing indicate combinations deviate severely). Their variation suggests that the observed deviations from Normality are not caused by inappropriate scaling and so indjividual outliers were sought. Using the above mentioned criteria resulted in detecting seven outliers (table 10-5). These were excluded together with the results for the other frequencies of those subjects.

The overall testing estimated $(-3.2 \pm 2.6) \times 10^{-2} \times$ age to be the effect of age on the coherence, which is insignificant ( $\mathrm{p}=0.237$; table 10-6). Neither did sex influence results significantly $(\mathrm{p}=0.143)$, and so both parameters can be excluded from

Tabic 10-5. Owtying response values for warious froguency - group combinations. Criterion was deviating 0.5 wnits of more from the Normal distribution as welli as lying distnetrom the bullk of data.

\begin{tabular}{|c|c|c|c|}
\hline diagnokis: & $\begin{array}{l}0.16 \\
12 \\
\end{array}$ & $\begin{array}{l}0.41 \\
\mathrm{~Hz}\end{array}$ & $\begin{array}{l}1 \times 15 \\
142\end{array}$ \\
\hline 0. controls &. & $\cdots$ & .. \\
\hline 1. Hitial stages of Meniere's syndrome & .. & $n$ & .. \\
\hline 2. peripheral westibular dysfumction, centrally welli compensated & 0.79 & 0.75 & an \\
\hline 3. peripheral vestibular dysfunction, cenbtrally poorly compensaled & $"$ & ". & .. \\
\hline 4. whiplash trauma & 0.53 & 0.73 & 0.55 \\
\hline 6. benign paroxysmal positioning dizziness & $\cdots$ &.. & $"$ \\
\hline F. vestibutar complaints from unknown causes & .. & . & -. \\
\hline
\end{tabular}




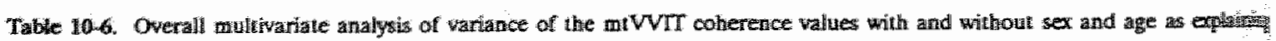

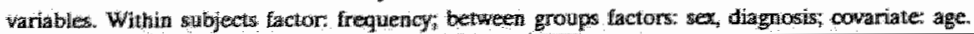

\begin{tabular}{|c|c|c|c|c|c|c|c|}
\hline & & \multicolumn{6}{|c|}{$\begin{array}{l}\text { significance of independent wariables } \\
\qquad(\mathrm{P}=\ldots)\end{array}$} \\
\hline & & age & $\begin{array}{l}\text { diagn. (afler } \\
\text { interaction) }\end{array}$ & $\begin{array}{l}\text { sex (after } \\
\text { interation) }\end{array}$ & $\begin{array}{l}\text { imteraction } \\
\text { of sex and } \\
\text { frequency }\end{array}$ & $\begin{array}{l}\text { interaction } \\
\text { of diagn. and } \\
\text { freq. }\end{array}$ & $\begin{array}{l}\text { effect of } \\
\text { freq (afk } \\
\text { interaction })\end{array}$ \\
\hline \multirow{2}{*}{$\begin{array}{l}\text { Indeptendent } \\
\text { varialdest } \\
\text { included for } \\
\text { analysis: }\end{array}$} & $\begin{array}{l}\text { age, distiosis, } \\
\text { sex, frequency }\end{array}$ & 0.237 & $0.055(t)$ & 0.143 & 0.345 & 0.307 & $<$ buons \\
\hline & $\begin{array}{l}\text { diagnosits, } \\
\text { freguency }\end{array}$ & $x$ & $0.065(t)$ & $-\infty$ & $-\infty$ & 0.244 & $<0,00006$ \\
\hline
\end{tabular}

(\$) suggestive, but not significant at $5 \%$ level

analysis safely.

Overall testing showed the diagnostically defined groups to differ only suggestively (p $=0.06$ ). If we accept a $6 \%$ chance for a type I error ${ }^{3}$ (instead of the usual $5 \%$ of less), groups can be compared pairwise. In fact, the overall testing is rather conservative, as it guards against drawing a wrong conclusion from comparing any group with any group. We, however, are interested in comparing any patient group with

Table 10-7. MANOVA results of pairwise comparison of VVIT colterence contrast walues from various patient groups anth control group ( $n \approx 6$ ), with diagnosis and stimulus frequency as independent variables and age as covariate. Stars indilutu importan. group differences.

\begin{tabular}{|c|c|c|c|c|}
\hline \multirow[t]{2}{*}{ diagnosis } & \multirow{2}{*}{$\begin{array}{l}\text { number } \\
\text { of } \\
\text { members }\end{array}$} & \multicolumn{3}{|c|}{$\begin{array}{c}\text { significance } \\
(p=\ldots)\end{array}$} \\
\hline & & $\begin{array}{l}\text { diagnosis } \\
\text { (after } \\
\text { interacilion) }\end{array}$ & $\begin{array}{l}\text { inveraction of } \\
\text { diagnosis and } \\
\text { treguency }\end{array}$ & $\begin{array}{l}\text { frequency } \\
\text { (after } \\
\text { interaction) }\end{array}$ \\
\hline 1. initial stage of Meniene's syndrome & 4 & $0.002+\star$ & $0.071(x)$ & 0.023 \\
\hline 2. peripheral westibular dystunction, well compensinted & 7 & $0.062(x)$ & $0.074(\hbar)$ & 0.010 \\
\hline 3. puripheral westibular itysfinction, poorly compensated & 13 & 9.031 t & 0.224 & 0.054 \\
\hline 4. whiplash trauma & 8 & 0.001 & $0.033 \star$ & 0.002 \\
\hline 6. benign paroxysmal positioning dizumess & 3 & $<0.0005+x$ & $0.003+x$ & $<0.0005$ \\
\hline 7. vestibulat complsints from unknown causes & 4 & $0.067(\omega)$ & 0.192 & 0.0036 \\
\hline
\end{tabular}

(t) stuggestive, but not significant at 5 \% level

$\star \quad$ significant al $5 \%$ level

$\star$ significant al low level

${ }^{3}$ Making a type I ermor means that one rejects the hypothesis that the obserwed differiences berween the compared growps ato due to chance only (and so interprets the differences as significant), white, in fact, the proups ane suatistically equivaluet Conversely, a type II error is made when one fails to resognize that the differences are significant. 


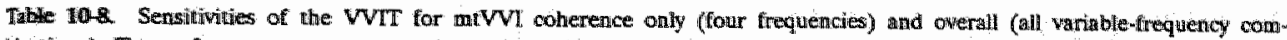

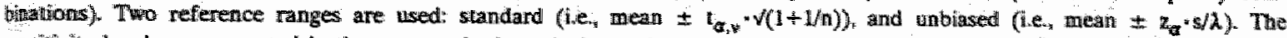

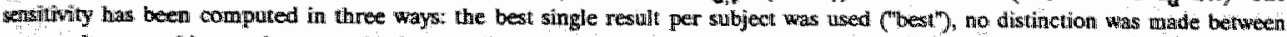

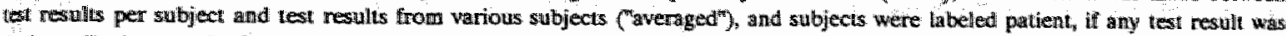

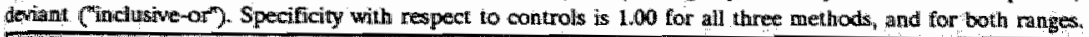

\begin{tabular}{|c|c|c|c|c|c|c|c|c|c|c|c|c|}
\hline \multirow[t]{2}{*}{ ditagnosis } & \multicolumn{6}{|c|}{ mtVVI coherence sensitivity } & \multicolumn{6}{|c|}{ owerall sensiturity } \\
\hline & best & $\begin{array}{l}\text { aver- } \\
\text { aged }\end{array}$ & $\begin{array}{l}\text { incl. } \\
\text { or }\end{array}$ & best & $\begin{array}{l}\text { aver- } \\
\text { aged }\end{array}$ & $\begin{array}{l}\text { incl } \\
\text { or }\end{array}$ & best & aged & incl: & best & $\begin{array}{l}\text { aver" } \\
\text { ageat }\end{array}$ & $\begin{array}{l}\text { hincl. } \\
\text { or }\end{array}$ \\
\hline d. Menivere & 1.00 & 0.88 & $1 .(0)$ & 1.00 & 0.94 & 1.00 & 1.00 & 0.51 & 1.00 & 1.00 & 0.60 & 1.00 \\
\hline 2. compensaned & 0.75 & 0.63 & 0.88 & 0.75 & 0.63 & 0.88 & 0.75 & 0.34 & 0.88 & 0.75 & 0.42 & 0.88 \\
\hline 3. uncompensated & 0.92 & 0.77 & 1,00 & 0.92 & 0.81 & 1.00 & 0.92 & 0.35 & 1.00 & 0.92 & 0.42 & 1.00 \\
\hline A. whiplash & 1.00 & 0.88 & 1.00 & 1.00 & 0.88 & 1.00 & 2.00 & 0.51 & 100 & 1.00 & 0.60 & 1.00 \\
\hline 6. BPP & 1.00 & 1.00 & 1.00 & 1.00 & 1.000 & 1.00 & 1.00 & 0.42 & 100 & 1.00 & 0.52 & 1.00 \\
\hline \multirow[t]{2}{*}{ 7. anknown } & 0.50 & 0.50 & 0.50 & 0.50 & 0.50 & 0.50 & 0.50 & 0.30 & 0.50 & 0.75 & 0.36 & 1.00 \\
\hline & \multicolumn{3}{|c|}{$\begin{array}{l}\text { slandard } \\
\text { reference range }\end{array}$} & \multicolumn{3}{|c|}{$\begin{array}{l}\text { unbiased } \\
\text { relerence range }\end{array}$} & \multicolumn{3}{|c|}{$\begin{array}{l}\text { standard: } \\
\text { reference range }\end{array}$} & \multicolumn{3}{|c|}{$\begin{array}{l}\text { unbiased } \\
\text { refrerance range: }\end{array}$} \\
\hline
\end{tabular}

controls only and therefore pairwise comparisons are also justified in case of a suggestive, but not yet significant, overall difference (Brown \& Hollander, p 238).

The results of pairwise testing are shown in table 10-7. From that table can be seen that for groups 2 and 7 there is insufficient evidence to conclude already that these

Table 10-9, Sensitivity and speciffcity (reganding non-patients with respeet to specific diagnosis) per diagnostic group and per treguency, susing standiard reference ranges. Minimal value of squared entor sum (undicated by gray celly gives optima! combination.

\begin{tabular}{|c|c|c|c|c|c|c|c|c|c|c|c|c|}
\hline & \multicolumn{4}{|c|}{ sensitionty } & \multicolumn{4}{|c|}{ specificity } & \multicolumn{4}{|c|}{$\begin{array}{l}\text { sum af } \\
\text { scualed deviations } \\
\text { from unity }\end{array}$} \\
\hline & $\begin{array}{r}0.16 \\
H z\end{array}$ & $\begin{array}{r}0.41 \\
\mathrm{~Hz}\end{array}$ & $\begin{array}{r}0.53 \\
\mathrm{H}_{2}\end{array}$ & $\begin{array}{r}1.15 \\
H x\end{array}$ & $\begin{array}{r}0.16 \\
\mathrm{~Hz}\end{array}$ & $\begin{array}{r}0.41 \\
\mathrm{~Hz}\end{array}$ & $\begin{array}{r}0.53 \\
\mathrm{~Hz}\end{array}$ & $\begin{array}{r}1.15 \\
\mathrm{H} 12\end{array}$ & $\begin{array}{r}0.16 \\
\mathrm{HL}\end{array}$ & $\begin{array}{r}0.41 \\
42\end{array}$ & $\begin{array}{r}0.53 \\
\mathrm{~Hz}\end{array}$ & $\begin{array}{r}1.15 \\
\mathrm{~Hz}\end{array}$ \\
\hline 1. Meniere & 1.00 & 1.00 & 0.75 & 0.75 & 0.30 & 0.32 & 0.45 & 0.32 & 0.50 & 0.46 & 036 & 0.53 \\
\hline 2. compensittedi & 0.75 & 0.50 & 0.50 & 0.75 & 0.28 & 0.25 & 0.43 & 0.33 & 0.59 & 0.81 & 0.58 & 0.52 \\
\hline 3. unicompensiated & 0.92 & 0.85 & 0.62 & 0.69 & 0.34 & 0.34 & 0.46 & 0.31 & 0.44 & 0.46 & 0.44 & 0.56 \\
\hline 4. whiplash & 0.80 & 100 & 0.70 & 1.00 & 0.29 & 0.37 & 0.47 & 0.39 & 0.54 & 0.40 & 0.37 & 0.37 \\
\hline 6. BPPD & 100 & 1.00 & 1,000 & 1,00 & 0,29 & $0.3 \mathrm{i}$ & 0.47 & 0.33 & $0.5 \pm$ & 0.47 & 0.28 & $0: 44$ \\
\hline 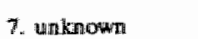 & 0.50 & 0.50 & 0.50 & 0.50 & 0.25 & 0.27 & 0.43 & 0.30 & 0.81 & 0.78 & 0.57 & 0.75 \\
\hline
\end{tabular}




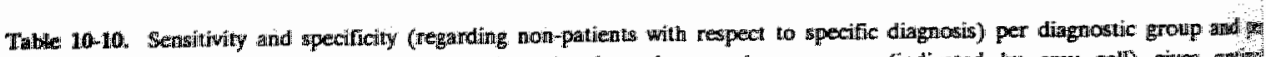

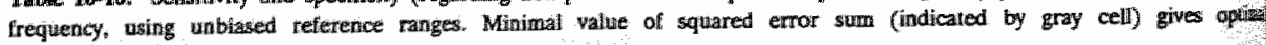
combiniationit.

\begin{tabular}{|c|c|c|c|c|c|c|c|c|c|c|c|c|}
\hline & \multicolumn{4}{|c|}{ sensitivity } & \multicolumn{4}{|c|}{ spectificty } & \multicolumn{3}{|c|}{$\begin{array}{l}\text { sumi of } \\
\text { squaned deviations } \\
\text { from unity }\end{array}$} & \\
\hline & $\begin{array}{r}016 \\
\mathrm{~Hz}\end{array}$ & $\begin{array}{r}0.41 \\
\mathrm{H} 2\end{array}$ & $\begin{array}{r}0.53 \\
\mathrm{~Hz}\end{array}$ & $\begin{array}{r}1.15 \\
\mathrm{HL}\end{array}$ & $\begin{array}{r}0.16 \\
\mathrm{~Hz}\end{array}$ & $\begin{array}{r}0.41 \\
\mathrm{~Hz}\end{array}$ & $\begin{array}{r}0.53 \\
\mathrm{~Hz}\end{array}$ & $\begin{array}{r}1.15 \\
H 2\end{array}$ & $\begin{array}{r}0.16 \\
\mathrm{~Hz}\end{array}$ & $\begin{array}{r}0.4 \mathrm{H} \\
\mathrm{HI}\end{array}$ & $\begin{array}{r}0.53 \\
H z\end{array}$ & $\begin{array}{r}\text { HLS } \\
\text { He }\end{array}$ \\
\hline 1. Meriere & 1.00 & 2.00 & 0.75 & 1.00 & 0.30 & 0.32 & 0.45 & 0.27 & 0.50 & 10.46 & 036 & $0 s^{2}$ \\
\hline 2, compensated & 0.75 & 0.50 & 0.50 & 0.75 & 0.28 & 0.25 & 0.43 & 0.25 & 0.59 & 0.81 & 0.58 & $a$ \\
\hline 3. uncompensied & 0.92 & 0.85 & 0.62 & 0.85 & 0.34 & 0.34 & 0.46 & 0.29 & 0.44 & 0.46 & 0.44 & 0.53 \\
\hline 4. whiplasth & 0.80 & 1.00 & 0.70 & 1.00 & 0.29 & 0.37 & 0.47 & 0.32 & 0.54 & 0.40 & 0.37 & a.tin? \\
\hline 6. $\mathrm{BPPD}$ & 1.00 & 1.00 & 1,00 & 1.00 & 0.29 & 0.31 & 0,47 & 0.27 & 0.51 & 0.47 & 028 & 0.54 \\
\hline 7. unknown & 0.50 & 0.50 & 0.50 & 0.50 & 0.25 & 0.27 & 0.43 & 0.23 & 0.81 & 0.78 & 0.57 & 0,85 \\
\hline
\end{tabular}

patients scored differently from the controls. But neither may one conclude that the) are identical to the controls, given the small sample sizes and the rather low chance probabilities $(p=0.062$ and $p=0.067$, respectively).

Compared to the controls, groups $1,3,4$, and 6 differ significantly $(\mathrm{p}=0.002, \mathrm{p}=$ $0.031, p=0.001$, and $p<0.0005$, respectively) in their overall responses and groups 4 and 6 also in their frequency-specific responses $(p=0.033$ and $p=0.003$, respet tively).

The clinical usefulness is assessed by calculating the sensitivities and specificities for the various diagnostic groups. Table 10-8 shows that using the unbiased reference ranges results in values that are only slightly better for the averaged sensitivities than when using the standard reference ranges. For the best single frequency result and for the "inclusive or" sensitivities, there is no difference between using the two kinds of reference ranges. Comparing the mtVVI coherence sensitivities with those calculated using all variable-frequency combinations, reveals that the first are better than the other variables, for the best single frequency sensitivities and the "inclusive or" sensitivities are the same for mtVVI coherences and the overall calculation, whild the average sensitivites are better for the mtVVI coherences. Only for the group with vestibular complaints of unknown origin is an other variable better.

Because with all of the approaches all controls scored normal, the specificity with respect to the controls remains 1.00 , and so does not influence the choice of the sensitivity criterium.

The specifities regarding non-patients with respect to a specific diagnosis are given in 
table 10-9, using the standard reference ranges, and in table 10-10, using the unbiased reference ranges. Also the sensitivities are given here. Using a smaller reference range can be expected to result in higher sensitivities. This is found here, but only for the highest frequency and only for two groups. The sensitivities for the other groupfrequency combinations remained the same. There is no expectation for how the specificity with respect to the non-group members (including other types of vestibular patients) will vary with the reference range width. Here, all six groups had an increased specificity for the highest stimulus frequency only. The conclusions are not affected by this change in reference range width: the group having the best combination of sensitivity and specificity is formed by the BPPD patients (sensitivity = 1.00 , specificity $=0.47$ ), followed by the whiplash patients (sensitivity $=0.70$, specificity $=0.47$ ).

\subsubsection{Individual contrasts of visuo-vestibular interaction with a visual target that is earth-fixed and with one that is moving asynchron- ously.}

In figures 10-7 and 10-8 the coherence contrasts (mean \pm standard error) are depicted per group and per chair-delivered stimulus frequency. Conform the initial

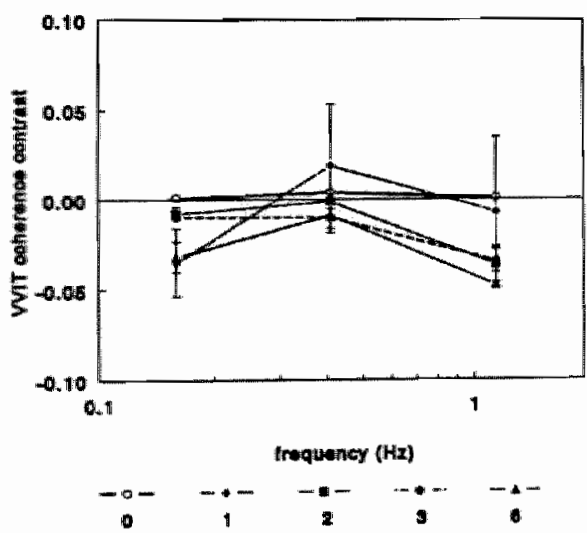

Fug. 10-7. Coherence contrast depending on sthmsis frequency for controls (o) and silgnifucantly different patients ( = Inimial Meniere, $=$ centrally well compersated perípheral vestibular dysfunction - = centrally poorly compensalled pertipheral vestibular dysfunction, axysmal positioning dizzinessy.

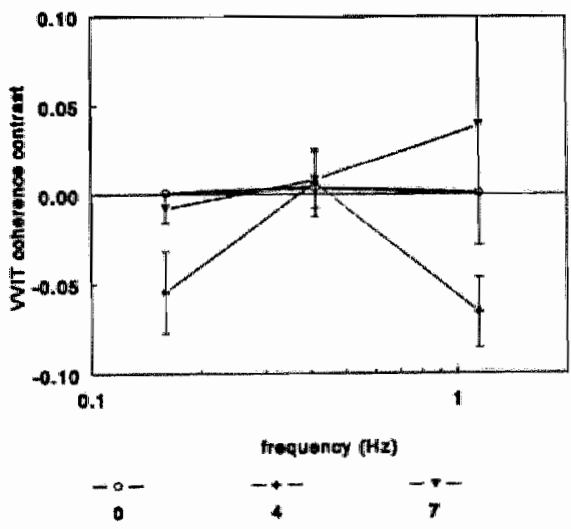

Fis. 10-8. Coherence contrast depending on stimulus fre quency for controts $(0)$ and linitially mot significantly different patients $(+$ (suggestively different) $=$ whiplash, $\cdot=$ unknown). 


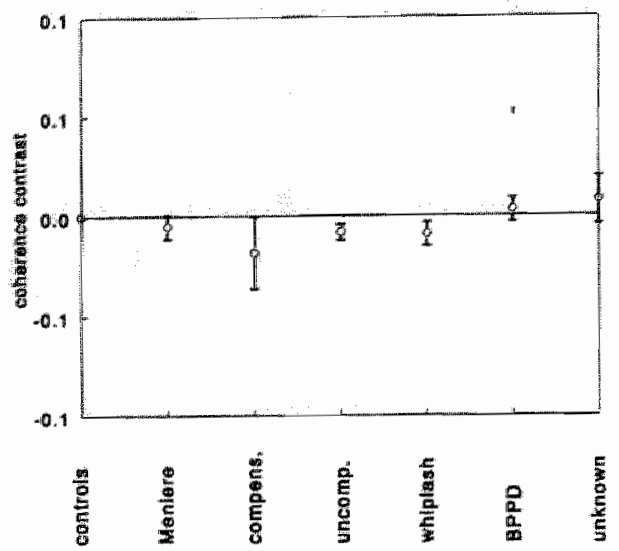

Fig. 10-9. Coherence relative differences between singltesime $(0.53 \mathrm{H} x)$ wisual target wowement, simultanous and a.synchronous with the sum-of-sines chatr movement, and idem: whithoul chair mowement. Same wertical scaling as figures. 107 and $10-8$ statistical analysis to be described belom the patient groups are divided in those who differed significantly from the corr trols, and those who did not, and outlying values are excluded. Figure 10-9 shows the coherence contrasts betweenthe purell visually delivered part of the mtVVI stimu lus and the corresponding SP stimulus. Odi average, the controls hardly differed bet. ween eftVVIT and mtVVIT (inferred from their coherence contrast being close to zero), while patients did. Patients generally responded poorer when the visual target moved too, but only so for $0.16 \mathrm{~Hz}$ and $1.15 \mathrm{~Hz}_{3}$ and not for $0.41 \mathrm{~Hz}$.

Because all groups performed best at 0.53 $\mathrm{Hz}$ (most values in figure $10-9$ are closet to zero than those in figures $10-7$ and 10 8), which was delivered by the movement of the visual target, and because frequency bins were averaged prior to calculating the coherence, the unaltered $0.41 \mathrm{~Hz}$ response might be an artefact of analysis.

The responses appeared to be non-Normal in Normal probability plots. Values for the parameter $\lambda$ for corrective Box-Cox transformations are given in table 10-11 (bold printing indicate combinations deviate severely). Their variation suggests that the observed deviations from Normality are not caused by inappropriate scaling and so individual outliers were sought. Using the above mentioned criteria resulted in

Table 10-11. Values for the parameter $\lambda$ of the Bow-Cox transformation for various frequency - group combinationat Bob printed walues indicale combindtions that deviate 0.5 units or more from the Normal distribution.

\begin{tabular}{|c|c|c|c|}
\hline dizagnosis & $\begin{array}{l}0.16 \\
142\end{array}$ & $\begin{array}{l}0.41 \\
\mathrm{~Hz} \\
\end{array}$ & $\begin{array}{l}1.1 .5 \\
\mathrm{~Hz} \\
\end{array}$ \\
\hline 0. controls & 0.60 & 230 & 82 \\
\hline 1. initial stages of Meniere's syndrome & 26 & 13 & 59 \\
\hline 2. perpheral vestibular onsurction, centrally well compensated & 42 & 2 & .5 .1 \\
\hline 3. peripheral vestithatir dysfunction, centrally poorly comperiusated & 7.9 & -4.2 & 0,5 \\
\hline 4. whipliash urauma & 8.9 & 3.9 & 4.8 \\
\hline 6. benign paroxys matal positioning dizziness & .50 & 210 & 0.60 \\
\hline 7. vestibular complaints from unkmown calsses & 38 & 38 & -10 \\
\hline
\end{tabular}


Tabte 10-12. Qunfing response values for various frequency-group combinations. Criterion was deviating 0.5 units or wore linom the Nommal distribution as well as lying distinct from the bulk of data.

\begin{tabular}{|c|c|c|c|}
\hline diagnosis & $\begin{array}{l}0.16 \\
\mathrm{~Hz}_{2}\end{array}$ & $\begin{array}{l}0.41 \\
\mathrm{~Hz}\end{array}$ & $\begin{array}{l}1.15 \\
412\end{array}$ \\
\hline 0. ocintrols & .. & $\therefore$ & 84 \\
\hline 1. finitial stages of Meniere's syndrome & $*$ & .. & $\ldots$ \\
\hline 2. peripherall westibular dysifunction, centrally well compensated & 0.081 & -0.138 & . \\
\hline 3. peripheral westibular dysfunction, centrally poorly compensated & 0.059 & 0.145 & $\cdots$ \\
\hline 4. whipiash trauma & 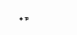 & $\cdots$ & .." \\
\hline 6. Henign paroxysralal poxitioning dizziness & $n$ & $\cdots$ & ". \\
\hline 7. Westibular complatimus from unknown causes & .. & 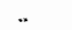 &. \\
\hline
\end{tabular}

detecting four outliers (table 10-12). These were excluded together with the results for the other frequencies of those subjects.

The overall testing (table $10-13)$ estimated $(-1.3 \pm 1.6) \times 10^{-2} \times$ age to be the effect of age on the coherence contrast, which is insignificant $(p=0.399)$. Sex influenced results significantly, depending on diagnostic group $(p=0.001)$, depending on frequency ( $p=0.011$ ), as well as depending on the combination of stimulus frequency and diagnostic group ( $\mathrm{p}<0.0005$ ). Because overall testing showed significant differences between groups, each patient group separately may be compared with the controls

The results of pairwise testing are shown in tables 10-14 and 10-15.

Sex is a suggestive, but not significant, factor for groups 6 and 7 only, so they may, and other groups should, be compared to controls without sex as an explaining variable. The results of the reduced MANOVA are given in table 10-15. The finding in table 10-14 that benign paroxysmal positioning dizziness patients and those with pestibular complaints of unknown origin might have a sex-specific response, is rather unexpected. Perhaps it can be explained as a freak outcome due to the small sample

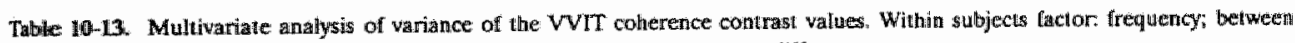
groups factors: sex, dagm;osis; covariate: age. Suars indicate important group differences.

\begin{tabular}{|c|c|c|c|c|c|c|c|}
\hline \multicolumn{8}{|c|}{$\begin{array}{l}\text { signifheance of independent variables } \\
\qquad(\mathrm{p}=\ldots)\end{array}$} \\
\hline age & $\begin{array}{l}\text { diagn. (after } \\
\text { interaction) }\end{array}$ & $\begin{array}{l}\text { sex (afied } \\
\text { interaction) }\end{array}$ & $\begin{array}{l}\text { interachion of } \\
\text { sex, diag. and } \\
\text { freq. }\end{array}$ & $\begin{array}{l}\text { inuteraction of } \\
\text { sex and diag. }\end{array}$ & $\begin{array}{l}\text { interaction of } \\
\text { sex and freq. }\end{array}$ & $\begin{array}{l}\text { interaction } \\
\text { of diagn. } \\
\text { and freq. }\end{array}$ & $\begin{array}{l}\text { effect of } \\
\text { freq. (afler } \\
\text { interactions) }\end{array}$ \\
\hline 0.399 & $<\frac{0,0005}{4 x}$ & 0.090 & $<0.0005$ & $\begin{array}{l}0,001 \\
+x\end{array}$ & 0.011 & $\begin{array}{c}0.0005 \\
+4\end{array}$ & 0.001 \\
\hline
\end{tabular}




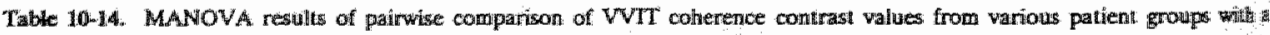

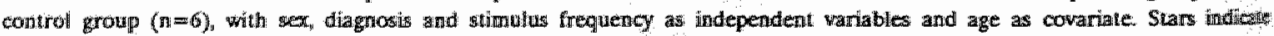
importan group differencies.

\begin{tabular}{|c|c|c|c|c|c|c|}
\hline \multirow{2}{*}{ difagnosis } & \multirow{2}{*}{$\begin{array}{l}\text { number } \\
\text { of } \\
\text { menbers }\end{array}$} & \multicolumn{5}{|c|}{ sigmificance $(p<\ldots)$} \\
\hline & & $\begin{array}{l}\text { diagniosis } \\
\text { (afier interac- } \\
\text { tion) }\end{array}$ & $\begin{array}{l}\text { interac- } \\
\text { tion) }\end{array}$ & $\begin{array}{l}\text { intierac- } \\
\text { tion of } \\
\text { sea and: } \\
\text { frequency }\end{array}$ & $\begin{array}{l}\text { interaction } \\
\text { of dian- } \\
\text { nosis and } \\
\text { freguency }\end{array}$ & $\begin{array}{l}\text { frequency } \\
\text { (afiter } \\
\text { interaco } \\
\text { tion) }\end{array}$ \\
\hline $\begin{array}{l}\text { 1. Mit jal stuges of Menietre's } \\
\text { zyndrome }\end{array}$ & 4 & 0.744 & 0.984 & 0.166 & $0.015 \times$ & 0.007 \\
\hline $\begin{array}{l}\text { 2. peripheral westibular dysi- } \\
\text { function, well compentasted }\end{array}$ & 6 & $0.026+$ & 0.746 & 0.543 & $0.060 \div$ & 0.005 \\
\hline $\begin{array}{l}\text { 3. peripheral vestibutlar dysi } \\
\text { function, partly compensated }\end{array}$ & 11 & $0.026 \div$ & 0.491 & 0.791 & $0.049+$ & 0.0100 \\
\hline 4. whiplasth trauma: & 10 & 0.103 & 0.585 & 0.754 & 0.209 & 0.125 \\
\hline $\begin{array}{l}\text { 6. besign paroxysmal positioning } \\
\text { dixoiness }\end{array}$ & 3 & $\begin{array}{l}<0.0005 \\
\text { int }\end{array}$ & 0.441 & 0.092 & $\begin{array}{l}0.001 \\
+1\end{array}$ & $<0.0005$ \\
\hline 7. unknowill & 4 & 0.540 & 0.197 & 0.057 & 0.237 & 0.016 \\
\hline
\end{tabular}

(*) suggestive, but not significant at $5 \%$ level

$+\quad$ significant at $5 \%$ level

$\star x$ significan at $1 \%$ level

Tabte 10-15. MANOVA results of painwise comparison of VVIT coberence contrast values from various patient groups whil i control group $(\mathrm{n}=6)$, with diagnosis and stimulus frequency as independent variables. Stars indicate important group differences.

\begin{tabular}{|c|c|c|c|c|}
\hline \multirow[t]{2}{*}{ diagnosis } & \multirow{2}{*}{$\begin{array}{c}\text { number } \\
\text { of merily } \\
\text { bers }\end{array}$} & \multicolumn{3}{|c|}{$\begin{array}{c}\text { significance } \\
(p=\ldots)\end{array}$} \\
\hline & & $\begin{array}{l}\text { diagnosuss } \\
\text { (alter } \\
\text { interaction) }\end{array}$ & $\begin{array}{l}\text { interaclion } \\
\text { of diagnosis } \\
\text { and fre } \\
\text { quency }\end{array}$ & $\begin{array}{l}\text { frequency } \\
\text { (afuer } \\
\text { interaction) }\end{array}$ \\
\hline 1. intitial sitages, of Mentere's symdrome & 4 & 0.744 & $0.016+$ & 0.007 \\
\hline 2. peripheral westibular dystumetion, well comperusated & 6 & $0.011 k$ & $0.033+$ & 0,003 \\
\hline 3. periphemal vesubular olysunction, partly compensated & 11 & $0.023 \times$ & 0.034 it & $0.00 \%$ \\
\hline 4. Whiplash trauma & 10 & $0.073(+)$ & 0.152 & 0,084 \\
\hline 6. bentgn paroxysmall positioning vertigo & 3 & $\begin{array}{c}<0.0005 \\
+x\end{array}$ & $\begin{array}{c}<0.0005 \\
+x\end{array}$ & $<0.0005$ \\
\hline 7. unkmown & 4 & 0.588 & 0.324 & 0.036 \\
\hline
\end{tabular}

(4) sugestive, bul not significant at $5 \%$ level

* significant at ses level

* * significant a 1 ge lewel 

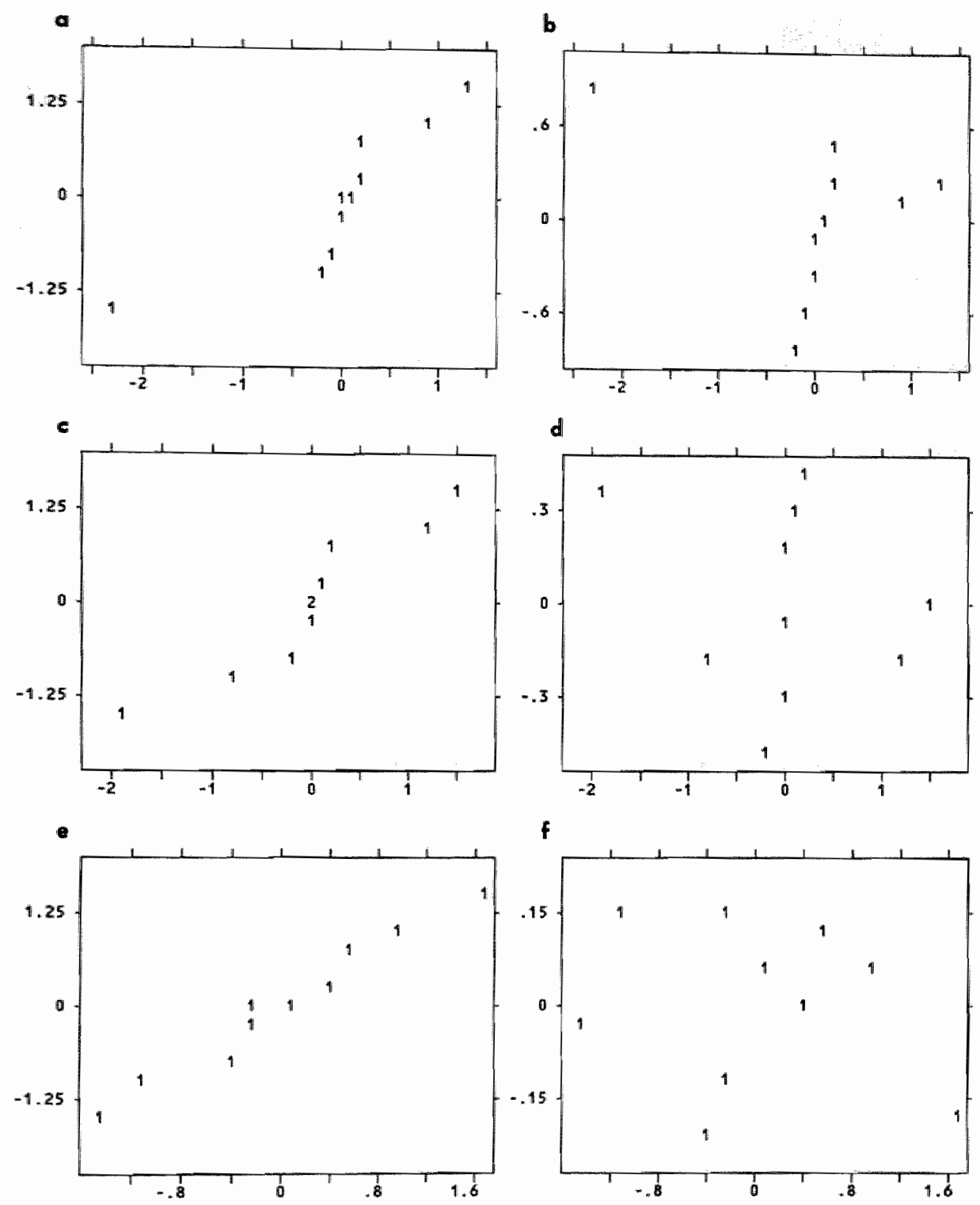

Future 10-110. Evaluation of the residusis from the MANOVA of the patients with intial Menlere (group 1) and the controls. a Normal probability plot for the constant. b. detrended Normat probatbility plot for the constami c. Normal probability plot for we second chair frequency $(\mathrm{f}=0.41 \mathrm{~Hz}$ ). di detrended Normal probability piot for the second eltuir frequency. e Normal probability plot for the third chair frequency $(f=1.15 \mathrm{~Hz})$. C detrended Normal probability plot for the thírd chair frequency. 

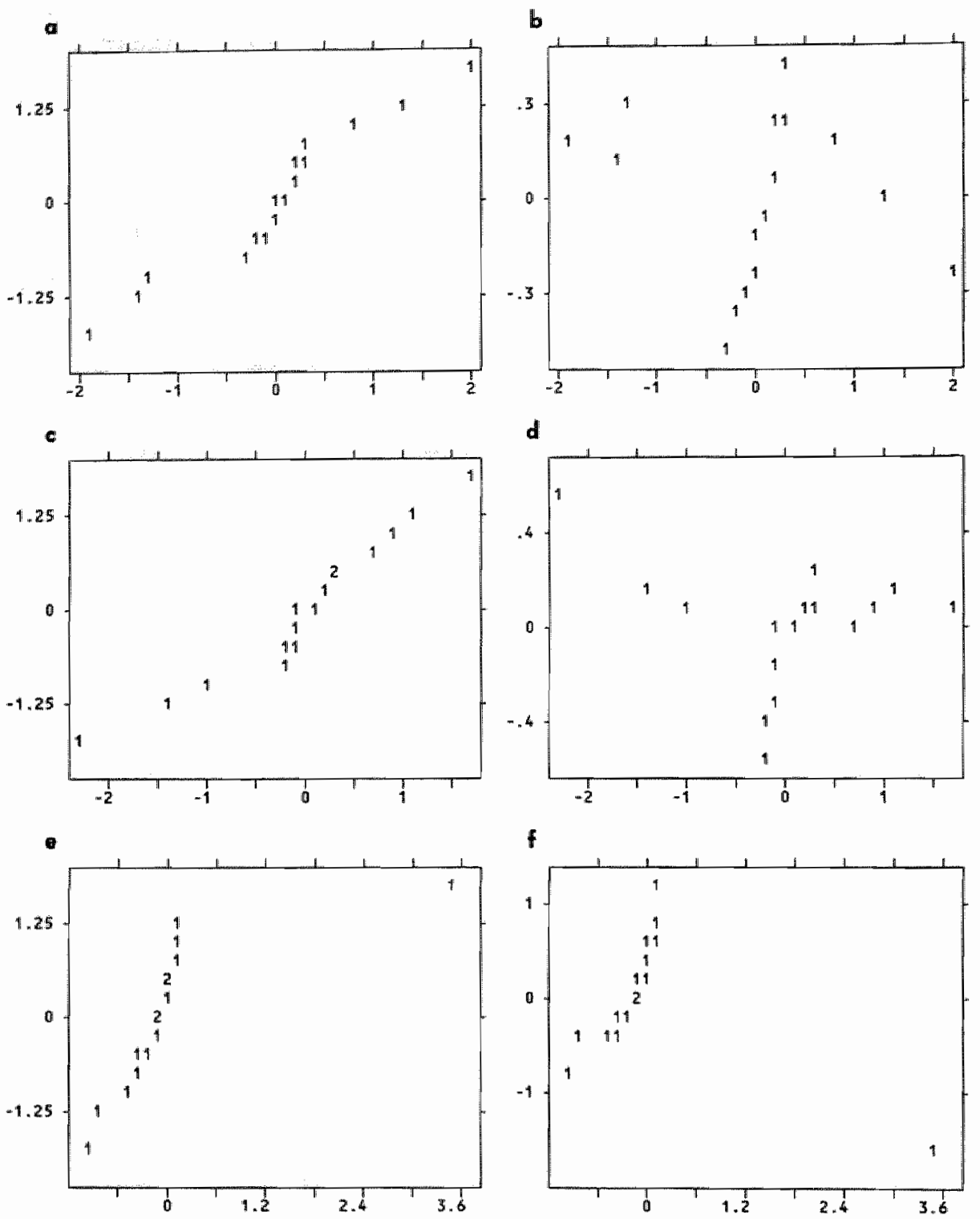

Figure 10-1L. Evaluation of the residuals from the MANOVA of the whiplash patients (groap 4) and the controls.

a. Normal probability plot for the constant. b. detrended Normal probability plot for the constant e Normal probability plot for the second chair frequency $(f=0.41 \mathrm{H}(2)$. d. detrended Normal probability plot for the second chair frequency. $c$ Normal probability plot for the thred chair frequency $(\mathbb{C}=1.15 \mathrm{~Hz})$, $\mathrm{E}$ detrended Nomal probability plot for the third chair frequemcy. 
sizes.

Compared to the controls, group 1 differs significantly $(p=0.016)$ in its frequencyspecific response but not in its overall response, while groups 2,3 , and 6 react in a significantly different way to the stimulus frequencies $(\mathrm{p}=0.033, \mathrm{p}=0.034$, and $\mathrm{p}$ $<0.0005$, respectively), as well as differ in overall response $(\mathrm{p}=0.011, \mathrm{p}=0.023$, and $p<0.0005$, respectively). Group 4 differs suggestively, but not significantly ( $p$ $=0.073$ ) from the controls with respect to overall response. So the suggestion by the means and the standard errors in figures 1 . and 2 that the whiplash patients (group 4)

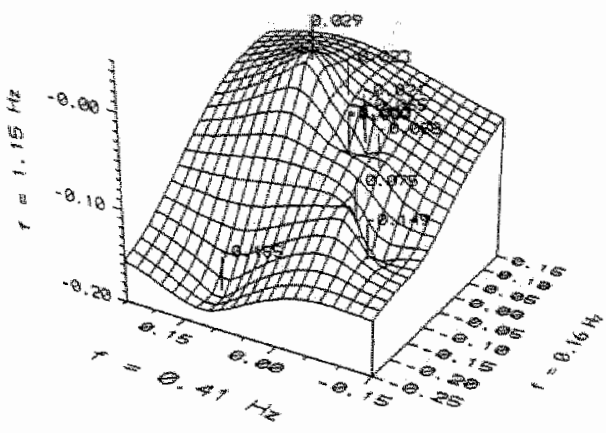

Fig. 10-12 Responses to the three clatir frequencies for the group of whiplash patients. Although each univariate dis uribution is approxinately Normal, the multivariate distributhon has one extreme oullier. differ from the controls at least as significantly as those with initial stages of Meniere's syndrome (group 1), is not substantiated by MANOVA.

In cases like these, it is worthwhile verifying whether all MANOVA requirements have been met. The univariate Normalities have already been checked (and would have been brought within reasonable limits by excluding outliers, if they had deviated too much). But the distributions should also be multivariately Normal, and this can be inspected by evaluating the Normality of each variable's residuals' distribution. The Normal probability plots and the detrended Normal probability plots of the residuals are given in figure 10-10 for the comparison of group 1 with the controls and in figure 10-11 for the comparison of group 4 with the controls. For the constant factor, group 1 has one outlier, making the distribution rather non-Normal (deviations within $[-0.8 \ldots 0.8]$ ) (figures $10-10 \mathrm{a}$ and $10-10 \mathrm{~b}$ ), while the residuals of the responses at 0.41 $\mathrm{Hz}$ are quite Normally distributed (figures $10-10 \mathrm{c}$ and $10-10 \mathrm{~d}$ ), and those of the responses at $1.15 \mathrm{~Hz}$ show very good Normality (figures $10-10 \mathrm{e}$ and 10-10f). In contrast, group 4 shows rather good Normality for the constant factor (figures 10-11a and $10-11 \mathrm{~b}$ ) and for the responses at $0.41 \mathrm{~Hz}$ (figures 10-11c and 10-11d), but has for the responses at $1.15 \mathrm{~Hz}$ one extreme outlier which makes the total distribution very non-Normal (figures $10-11 \mathrm{e}$ and $10-11 \mathrm{f}$; deviations within $[-1.6 \ldots 1.2]$ ). So the fact that the whiplash patients differ only suggestively from the controls, according to MANOVA, might be due to one multivariate outlier. To identify that outlier, the data for group 4 has been plot in a perspective way in figure 10-12. And indeed, one triplet (i.c., $\{-0.203,0.117,-0.185\}$ ) is lying completely beside the bulk of the data. Repeating the MANOVA for the whiplash patients versus the controls, with the multivariate outlier removed, produced the results shown in table 10-16. As expected, 


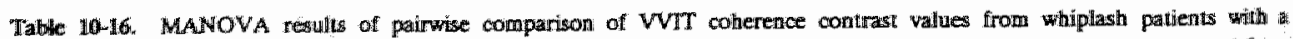

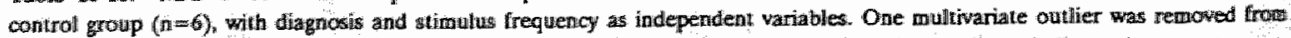
the patientis' poptulation, and therefore these results replace those given in mable 10-12. Stars indicate injportant growg differencien.

\begin{tabular}{|c|c|c|c|c|}
\hline \multirow[t]{2}{*}{ diagnosis } & \multirow{2}{*}{$\begin{array}{l}\text { number of } \\
\text { memibers. }\end{array}$} & \multicolumn{3}{|c|}{$\begin{array}{c}\text { significano } \\
(p=\ldots)\end{array}$} \\
\hline & & $\begin{array}{l}\text { diagnasis } \\
\text { (afler } \\
\text { interaction) }\end{array}$ & $\begin{array}{l}\text { interaction of } \\
\text { diagnosis and } \\
\text { frequency }\end{array}$ & $\begin{array}{l}\text { firequency } \\
\text { (after } \\
\text { interaction) }\end{array}$ \\
\hline 4. whiplash traumb & 9 & 0.116 & $0.006 \times k$ & 0.001 \\
\hline
\end{tabular}

† sigmificant al $1 \%$ level

the differences are now statistically significant.

Returning to table 10-15, it can be seen that group 7, finally, does not distinct itself from the controls. In conclusion, five groups of patients (those with initial stages of Meniere's syndrome, those with a well and those with a incompletely compensated peripheral vestibular dysfunction, those having whiplash trauma and those with benign paroxysmal positioning vertigo) produce deviant VVIT coherence contrast values, and one group (those for whom the causes of their vertigo complaints are unknown) produces normal VVIT coherence contrast values.

The clinical usefulness is assessed by calculating the sensitivities and specificities for the various diagnostic groups. Table 10-17 shows that using the unbiased reference ranges results in values that are better for the averaged sensitivities than when using

Tlable 10-17. Sensitivities of the VVIT for coherence contrast only (four frequencies) and overail (all variable-frequency coma-" binations). "Two reference ranges are tused" standard (i.e., njean $\pm t_{\alpha, v} \cdot v(1+1 / n)$ ), and unbiased (i.e, mean $\left.\pm z_{\alpha} \cdot s / \lambda\right)$, The sensitivity has been computed in three ways: the besit single result per subject was, used (best"), no distinction was made between test restilts per subject and test results from various subjects ("aweraged), and subjects were labeled patient, if any test result was. deviant (inclusiwe-or). Specificity with respect to controls is 1.00 for all three methods and for both ranges.

\begin{tabular}{|c|c|c|c|c|c|c|c|c|c|c|c|c|}
\hline \multirow[t]{2}{*}{ diangnosils } & \multicolumn{6}{|c|}{ coherence contrast sensitivity } & \multicolumn{6}{|c|}{ overall sensitivity } \\
\hline & best: & $\begin{array}{l}\text { aver- } \\
\text { aged }\end{array}$ & $\begin{array}{l}\text { incl. } \\
\text { or }\end{array}$ & bet & $\begin{array}{l}\text { awer- } \\
\text { aged }\end{array}$ & $\begin{array}{l}\text { incl. } \\
\text { or }\end{array}$ & best. & $\begin{array}{l}\text { aver- } \\
\text { aged }\end{array}$ & $\begin{array}{l}\text { inct. } \\
\text { or }\end{array}$ & best: & aver- & $\begin{array}{l}\text { incl. } \\
\text { or }\end{array}$ \\
\hline 1. Meniere & 1.00 & 0.94 & 1.00 & 1000 & 0.94 & 1.00 & 1.00 & 0.51 & 1.000 & 1.00 & 0.66 & $\mathbb{1 1}, 00$ \\
\hline 2. comperusa ted & 0.75 & 0.31 & 0.75 & 0.75 & 0.44 & 0.88 & 0.75 & 0.34 & 0.88 & 0.75 & 0.42 & 0.88 \\
\hline 3. uncompensated & 0.62 & 0.44 & 0.69 & 0.77 & 0.52 & 0.92 & 0.92 & 0.35 & 1.00 & 0.92 & 0.42 & 1.00 \\
\hline 4. whiplash & 0.80 & 0.70 & 0.90 & 1.00 & 0.75 & 1.00 & 1.00 & 0.51 & 1.00 & 100 & 0.60 & 1.00 \\
\hline 5. BPPD & 1.00 & 0.75 & 1.00 & 1.00 & 0.75 & 1.00 & 1.00 & 0.42 & 1.00 & 1.00 & 0.52 & 1.00 \\
\hline 6. Mrtknown & 0.50 & 0.44 & 0.50 & 0.75 & 0.50 & 0.75 & 0.50 & 0.30 & 0.50 & 0,75 & 0.36 & 1.000 \\
\hline & \multicolumn{3}{|c|}{$\begin{array}{l}\text { standaud } \\
\text { reference range: }\end{array}$} & \multicolumn{3}{|c|}{$\begin{array}{l}\text { umbiased } \\
\text { reference range }\end{array}$} & \multicolumn{3}{|c|}{$\begin{array}{l}\text { standard } \\
\text { neference range }\end{array}$} & \multicolumn{3}{|c|}{$\begin{array}{l}\text { unbiased } \\
\text { reference range }\end{array}$} \\
\hline
\end{tabular}




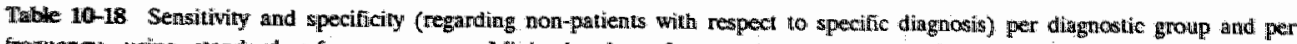
firequency; using standard reference ranges. Minimal valwe of squared error sum (indicated by gary oell) gives optims motmbintation.

\begin{tabular}{|c|c|c|c|c|c|c|c|c|c|c|c|c|}
\hline & \multicolumn{4}{|c|}{ sensitivity } & \multicolumn{4}{|c|}{ specificity } & \multicolumn{4}{|c|}{$\begin{array}{l}\text { sum of } \\
\text { squared deviations } \\
\text { drom unity }\end{array}$} \\
\hline & $\begin{array}{r}0.16 \\
\mathrm{~Hz}\end{array}$ & $\begin{array}{r}0.41 \\
\mathrm{~Hz}\end{array}$ & $\begin{array}{r}0.53 \\
\mathrm{~Hz} \\
\end{array}$ & $\begin{array}{r}1.15 \\
142\end{array}$ & $\begin{array}{r}0.16 \\
\mathrm{~Hz} \\
\end{array}$ & $\begin{array}{r}0.41 \\
\mathrm{~Hz}\end{array}$ & $\begin{array}{r}0.53 \\
\mathrm{~Hz} \\
\end{array}$ & $\begin{array}{r}1.15 \\
\mathrm{~Hz}\end{array}$ & $\begin{array}{r}0.16 \\
H 12\end{array}$ & $\begin{array}{r}0.41 \\
\mathrm{H} \\
\end{array}$ & $\begin{array}{r}0.53 \\
1 \%\end{array}$ & $\begin{array}{r}1.115 \\
\mathrm{~Hz}\end{array}$ \\
\hline 1. Meniere & 0.75 & 100 & 1.00 & 1.000 & 0.57 & 0,68 & 0.61 & 0.39 & 0.25 & 010 & 0.15 & 0.38 \\
\hline 2 compensated & 0.25 & 0.13 & 0.13 & 0.75 & 0.50 & 0.58 & 0.50 & 0.38 & 0.81 & 0.95 & 1.02 & 0.45 \\
\hline 3. uncompensater & 0.46 & 0.31 & 0.38 & 0.62 & 0.54 & 0.60 & 0.54 & 0.34 & 0.50 & 0.64 & 0.59 & 0.58 \\
\hline 4. Mhüplash & 0.70 & 0.60 & 0.70 & 0.80 & 0.61 & 0.68 & 0.63 & 0.39 & 0.25 & 0.26 & 0.23 & 0.41 \\
\hline 6. BPPD & 100 & 0.33 & 0,67 & 1.00 & 0.58 & 0.62 & 0.58 & 0.38 & 0.18 & 0.59 & 0.29 & 0.39 \\
\hline 7. unkenown & 0.25 & 0.50 & 0.50 & 0.50 & 0.52 & 0.64 & 0.57 & 0.34 & 0.79 & 0.38 & 0.44 & 0.68 \\
\hline
\end{tabular}

the standard reference ranges. Comparing the coherence contrast sensitivities with those calculated using all variable-frequency combinations, reveals that the first are better than most other variables (for the averaged sensitivities are higher), but not the best for the group of centrally poorly compensated peripheral vestibular patients (for their best single frequency sensitivities using all variable-frequency combinations is higher than the best coherence contrast sensitivity).

Because with all of the approaches all controls scored normal, the specificity with re-

Table 10-19. Sensitivity and specificity (reggandigg nom-patients with nespect to specific diagnosis) per diagnostic group and per finequency; using unbiased refierenoe ianges. Minimal vilue of squated error sum (indicated by gray cell) gives optimal combination.

\begin{tabular}{|c|c|c|c|c|c|c|c|c|c|c|c|c|}
\hline & \multicolumn{4}{|c|}{ sensitivity } & \multicolumn{4}{|c|}{ specificity } & \multicolumn{4}{|c|}{$\begin{array}{l}\text { sum of } \\
\text { squared deviations } \\
\text { from uniny }\end{array}$} \\
\hline & $\begin{array}{r}0.16 \\
\mathrm{~Hz}\end{array}$ & $\begin{array}{r}0.41 \\
\mathrm{~Hz}\end{array}$ & $\begin{array}{r}0.53 \\
\mathrm{H} . \mathrm{X}\end{array}$ & $\begin{array}{r}1.15 \\
\mathrm{~Hz}\end{array}$ & $\begin{array}{r}0.16 \\
\mathrm{~Hz}\end{array}$ & $\begin{array}{r}0.41 \\
1 \mathrm{~Hz}\end{array}$ & $\begin{array}{r}0.53 \\
\mathrm{~Hz}\end{array}$ & $\begin{array}{r}1.15 \\
12\end{array}$ & $\begin{array}{r}0.16 \\
164\end{array}$ & $\begin{array}{r}0.4 \mathrm{H} \\
\mathrm{HH}\end{array}$ & $\begin{array}{r}0.53 \\
\mathrm{~Hz}\end{array}$ & $\begin{array}{r}1.15 \\
\text { HHiz } \\
\end{array}$ \\
\hline 1. Meniere & 0.75 & 100 & 1.00 & 1.00 & 0.48 & 0,64 & 0.61 & 0.27 & 0.34 & 013 & 0.15 & 0,53 \\
\hline 2. compensated & 0.50 & 0.38 & 0.1 .3 & 0.75 & 0.45 & 0.58 & 0.50 & 0.25 & 0.55 & 0.57 & 1.02 & 0.63 \\
\hline 3. uncompensaled & 0.152 & 0.31 & 0.38 & 0.77 & 0.49 & 0.54 & 0.54 & 0.26 & 0.41 & 0.69 & 0.59 & 0.61 \\
\hline 4. whiplash & 0.70 & 0.60 & 0.70 & 1.00 & 0.50 & 0.63 & 0.63 & 0.32 & 0.34 & 0.30 & 023 & 0.47 \\
\hline 6. $\mathrm{BPPD}$ & 1.00 & 0.33 & 0.67 & 100 & 0.49 & 0.58 & 0.58 & 0.27 & 0.26 & 0.62 & 0.29 & 0.54 \\
\hline 7. & 0.25 & 0.50 & 0.50 & 0.75 & 0.43 & 9.59 & 0.57 & 0.25 & 0.89 & 0.42 & 0.44 & 0.63 \\
\hline
\end{tabular}




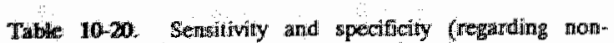
patherte whth tespect to specific diagnisisis for the com-

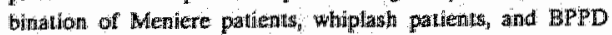
patientu. Stin, freq stimulus freguency, error sum = sum of squared deviations from unity.

\begin{tabular}{|c|c|c|c|}
\hline $\begin{array}{l}\text { stim. freg } \\
\text { (hos) }\end{array}$ & sensitivity & specificity & $\begin{array}{l}\text { ermor } \\
\text { Stiti }\end{array}$ \\
\hline 0,16 & 0.76 & 0.71 & 0,14 \\
\hline 0.41 & 0.65 & 0.77 & 0.18 \\
\hline 0.53 & 0.76 & 0.74 & 0.13 \\
\hline 1.15 & 0.88 & 0.48 & 0.28 \\
\hline
\end{tabular}

Table 10-21. Effect of combining the groups of Mfeniere patients, whiptasis patiens and BPPD patienus on sentilnity and speciticity foased on the three lower stimulus frequen cies). Error sum = sum of squared devations from unim group average $=$ weighted group averages of frequench combined values, combined groups = frequency aneraget wallues of combined groups:

\begin{tabular}{|l||c|c|c|}
\hline & sensitivity & specificiny & $\begin{array}{l}\text { efror } \\
\text { sum }\end{array}$ \\
\hline group anerage & 0.73 & 0.62 & 0.22 \\
\hline $\begin{array}{l}\text { combined } \\
\text { group }\end{array}$ & 0.72 & 0.74 & 0.15 \\
\hline
\end{tabular}

spect to the controls remains 1.00 , and so does not influence the choice of the sensitivity criterium.

The specifities regarding non-patients with respect to a specific diagnosis are given in table 10-18, using the standard reference ranges, and in table 10-19, using the unbiased reference ranges. Also the sensitivities are given here. Using a smaller reference range can be expected to result in higher sensitivities. This is found here for some group-frequency combinations, while the sensitivities for the other combinations remained the same. There is no expectation for how the specificity with respect to the non-group members (including other types of vestibular patients) will vary with the reference range width. Here, the specificities for almost all chairdelivered frequencies decreased, while those for the purely visually delivered frequency remained the same. The conclusions are not greatly affected by this change in reference range width: the group having the best combination of sensitivity and specificity is formed by the Meniere patients (sensitivity $=1.00$, specificity $\approx 0.65$ ), followed by the whiplash patients (sensitivity $=0.70$, specificity $=0.63$ ), and the BPPD patients (sensitivity $=1.00$, specificity $=0.58$ using the standard reference range and 0.49 using the unbiased one).

In these best combinations, the specificity is rather poor. This might result from the three groups reacting in the same way, and therefore the sensitivities and specificities are also calculated for the combined group of Meniere patients, whiplash patients, and BPPD patients. And indeed, table 10-20 shows increased specificities (at the cost, however, of decreased sensitivities). This table suggests even more clearly than table 10-18 that the responses to the three lower frequencies are similar, while that to the highest frequency result in high sensitivities and low specificities. When the results of the three lower frequencies are combined, a sensitivity of 0.72 , and a specificity of 0.74 is obtained. In table 10-21 these frequency-averaged sensitivity and specificity for 
the combined group are compared to the weigthed group-averaged sensitivity and specificity for the combined frequencies. Combining the three groups increased the specificity and left the sensitivity intact.

\subsubsection{Conclusions.}

The multivariate comparison of group means, and the sensitivity values agree with each other. Both rank the investigated, diagnostically defined groups as follows: BPPD, whiplash, Meniere, centrally poorly compensated peripheral vestibular dysfunction, centrally well compensated peripheral vestibular dysfunction, and vestibular complaints from unknown origin (in order of decreasing difference with normals). Only for coherence contrast, MANOVA places the group of well compensated patients before the Meniere patients. Apart from the group with unknown causes, all group differed significantly $(p<0.025)$ from the controls.

For the highest stimulus frequency $(1.15 \mathrm{~Hz})$ for coherence contrast, and for all frequencies for mtVVI coherence, sensitivities are high $(0.62 .1 .00)$ for all groups except those with unknown causes. For the three lower stimulus frequencies $(0.16$, 0.41 , and $0.53 \mathrm{~Hz}$ ), coherence contrast sensitivities are high for Meniere patients (0.92), whiplash patients (0.67), and BPPD patients (0.67), but low for compensated peripheral vestibular patients $(0.17)$, and for uncompensated peripheral vestibular patients (0.38).

The mtVVI coherence is found to be the most sensitive variable, compared to gain contrast, mtVVI phase shift, phase shift difference, and coherence contrast.

Specificity with respect to normals is 1.00 for both the standard reference ranges and the unbiased reference ranges.

When the specificity is calculated for other vestibular patient groups as well as normals (instead of for normals only), i.e., when the VVIT is to be used for differential diagnosis, then a slightly different order emerges than given above. The groups having the best combinations of sensitivity and specificity, are then those having Meniere's disease, BPPD, and whiplash (for both the mtVVI coherence and the contrast between miVVI and eftVVI coherence; the contrast has the best sensitivity $\&$ specificity combinations: $1.00 \& 0.68,1.00 \& 0.58$, and $0.70 \& 0.63$, respectively). The frequency-specific differences between vestibular patients may be due to chance, and so pooling these data may be helpful. Combining the three lower stimulus frequencies and combining the groups of Meniere patients, whiplash patients, and BPPD patients, lead to a coherence contrast sensitivity of 0.73 , and a coherence contrast specificity (with respect to other vestibular patients and controls) of 0.74 . 
At the highest stimulus frequency, all patients groups except those for whom no causes are known, score rather deviantly, and consequently the specificity with respect to other vestibular patients is low. 


\section{Discriminating power of the Visuo-Vestibular Interaction Test for type of pathology, compared to those of standard tests. \\ 11.1 Abstract.}

In previous chapters $(5,10)$ a vestibular function test (named Visuo-Vestibular Interaction Test: VVIT) was presented, in which a visual target may move asynchronously with a rotatory chair. The variables gain, phase shift, and coherence were there discussed, as well as the resulting univariate sensitivity and specificity. The current chapter investigates how well the VVIT differentiates between various groups of patients and this is compared to the performance of a combination of standard tests. Weights for a combinatorial function were obtained by discriminant analysis. Employing this function to categorize the same subjects as were used to derive that function, allowed classification in accordance with the diagnoses in $86 \%$ of the cases using VVIT results and anamneses, and in $72 \%$ using standard tests and anamneses. The better performance of the VVIT was in spite of the fact that the diagnoses were based on standard test results (and anamneses).

The patient groups best recognized by VVIT results and anamneses were those having Meniere's syndrome (100\%), those having BPPD (100\%), and those having whiplash trauma $(90 \%)$. The latter two were also best recognized using standard test results and anamneses (100\% and $89 \%$, respectively). The recognition of whiplash patients on basis of their VVIT results is rather independent from prior knowledge whether or not all subjects are patients, while this might be important for standard test based recognition.

\subsection{Introduction.}

A full introduction of the VVIT was given earlier (chapter 5: "Effect of asynchronous visual stimulation on the Vestibulo-Ocular Reflex gain of normals"), so here only the essentials will be repeated.

In order to elude the variation inherent to measuring the vestibulo-ocular reflex (VOR) in the dark, a visual target is presented. The usual ways in which the resulting visuo-vestibular interaction (VVI) is studied, (namely, by offering either an earth-fixed target or a chair-fixed target) allow, but may not require, the two systems to interact. To force an interaction between smooth pursuit (SP) and VOR, we use a visual target which moves asynchronously with the chair. Predictive effects are reduced by having the chair move in a pseudo random (sum-of-sines) way. As both visual target movement and chair movement are below the SP upper limit, we expect normals and patients to perform equally well to either one of them. But because the combination 
is above the SP upper limit, we expect patients to perform worse than normals to the combined stimulus, assuming that they will overburden their SP system in an effort to compensate for the insufficient vestibular function. By subtracting the visuo-vestibulaty interaction with an earth-fixed target (eftVVI; i.e., visually enhanced VOR) stimulus and the response to it, from the visuo-vestibular interaction with an asynchronousily moving target (mtVVI) stimulus and the response to it, the visual components are obtained. It is hypothesized that the vestibular function is reflected by the ratio of the smooth and saccadic eye movements (and thus by the gain of the median-filtered response). Different phase shifts for normals and patients and for eftVVI and mtWI might be expected in relation to the complexity of the stimulus and the type of neural processing it is subjected to.

\subsection{Material and methods. \\ 11.3.1 Subjects.}

Data were obtained from the subjects who were described earlier (see chapter 8). in short, 42 patients (median age: 45) and 6 controls (median age: 30) were examined. The patients felt in 6 categories: those having initial stages of Meniere's syndrome (4), those having peripheral vestibular dysfunctions, centrally well compensated (8) and centrally partly compensated (13), whiplash victims (10), BPPD patients (3), and those for which the causes of their complaints are unknown (4). They were diagnosed by an experienced vestibulologist (dr. Kingma), using anamneses and results of standard tests (see below). All subjects were tested without optical corrections.

\subsubsection{Experimental set-up.}

The experimental set-up was as reported previously (chapter 5), and so only a brief description follows here. Subjects sat in a rotary chair moving in a pseudo random way (sum-of-sines: $0.16,0.41$, and $1.15 \mathrm{~Hz}$, each with a maximum velocity of $10 \% / \mathrm{s})_{1}$ while watching a dot which was either earth-fixed or moving independently $(0.53 \mathrm{~Hz}$ with a maximum velocity of $32 \% / \mathrm{s}$ ). The eye position was measured using infra red reflection, and the head velocity was monitored with an angular rate sensor. Eye position, head velocity, and visual target position were sampled at a $50 \mathrm{~Hz}$ frequency for 125 seconds by a 12 bit analog-to-digital (AD) converter (the first two after being filtered analogously with a cut-off frequency of $10 \mathrm{~Hz}$ ) and were digitally stored for offline analysis. 
Standard tests consisted of calorization $\left(30^{\circ}\right.$ Celsius and $44^{\circ}$ Celsius, in the order right cold, left cold, left warm, right warm), torsion in the dark $(0.1 \mathrm{~Hz}$ frequency, 50 is peak velocity), a smooth pursuit test $\left(0.29 \mathrm{~Hz}\right.$ frequency, $15^{\circ}$ amplitude), and an optokinetic test $(10,20,30$, and $40 \%$ /s velocity, both horair and anti-horair). Left and right horizontal eye position was measured by electro-oculography, and was sampled at $100 \mathrm{~Hz}$

\subsubsection{Digital signal analysis.}

Processing and analysis of the signals was as reported before (see chapters 5,6 , and 7). In summary, position data were digitally differentiated to velocities, after which the total stimulus was combined from visual target movement and head movement: Fast components and eye blink artefacts were removed by a median filter. Processing was different for calculating gain and coherence at the one hand, and phase shift at the other. For gain and coherence, the width of the median filter was $0.15 \mathrm{~s}$. For each recording a gain and a coherence spectrum was calculated from averaged power spectra, obtained by combining the power spectra from 5 half overlapping, Welchwindowed time segments. The coherence spectrum was further frequency-averaged. In order to calculate the phase shift over the same time length as gain and coherence were, the digitally stored signal was resampled, which made a different width of the median filter optimal: $0.35 \mathrm{~s}$. The values at the response frequencies were extracted automatically from the spectra, using preset criteria.

\subsubsection{Statistical analysis.}

Discriminant analysis was performed using SPSS software (SPSS/PC+, Chicago, USA). Because this technique assumes Normal distributions, univariate outliers (as identified in the chapters 8,9 , and 10 , using Normal probability plots) were removed from the data. Deleting all subjects with outlying or missing values, however, would decimate the available data rather much (because so many variables are considered simultaneously), and so removed or missing continuous values were replaced by the group average for that variable. Cases with missing dichotomous variables had to be excluded entirely, because inserting the group averages would mean introducing fractional values.

When pairs of variables had a correlation of more than 0.6 , then one of them was excluded from analysis. Further details are given in the text. 


\subsection{Results and discussion.}

The contribution to the discriminant function of highly correlated varibles is shared, making the individual coefficients meaningless. Therefore, when pairs of variables had a correlation of more than 0.6 (i.e., in the $[0.5500 .1 .0000]$ range), then one of them was excluded from analysis. Table 11-1 shows the found relevant correlations of the test and anamnestic variables for the VVIT, and table 11-2 for the standard testis (only variables having correlations exceeding the threshold are included in the tables, and of them; only the exceeding values are shown).

Remowing correlated variables was done iteratively for the VVIT. The variable having the highest number of rellevant correlations was removed first and then the new numbers of correlations per variable were scored. For the standard tests, the derived

Table 11 - L. Correlation table of VVIT variables (only variables having a correlation of 0.55 or more are shown); gc $=$ gati contrast, eft ph(ase) = effVVTT phase stift, mt ph(ase) = miVVIT phase shift, ph(ase) diff(erence) = phase shift differente; coh(erence) $=$ efiVVIT coherence, mi coh(erence) $=$ mVVT coherence, $c c=$ coberence contrast, $\mathrm{fI}=0.16 \mathrm{~Hz}$ (chair) $\mathrm{L}=$ $0.41 \mathrm{~Hz}$ (chair) $\mathrm{B}=0.53 \mathrm{~Hz}$ (visual target), $\mathrm{f} 4=1.15 \mathrm{~Hz}$ (chair).

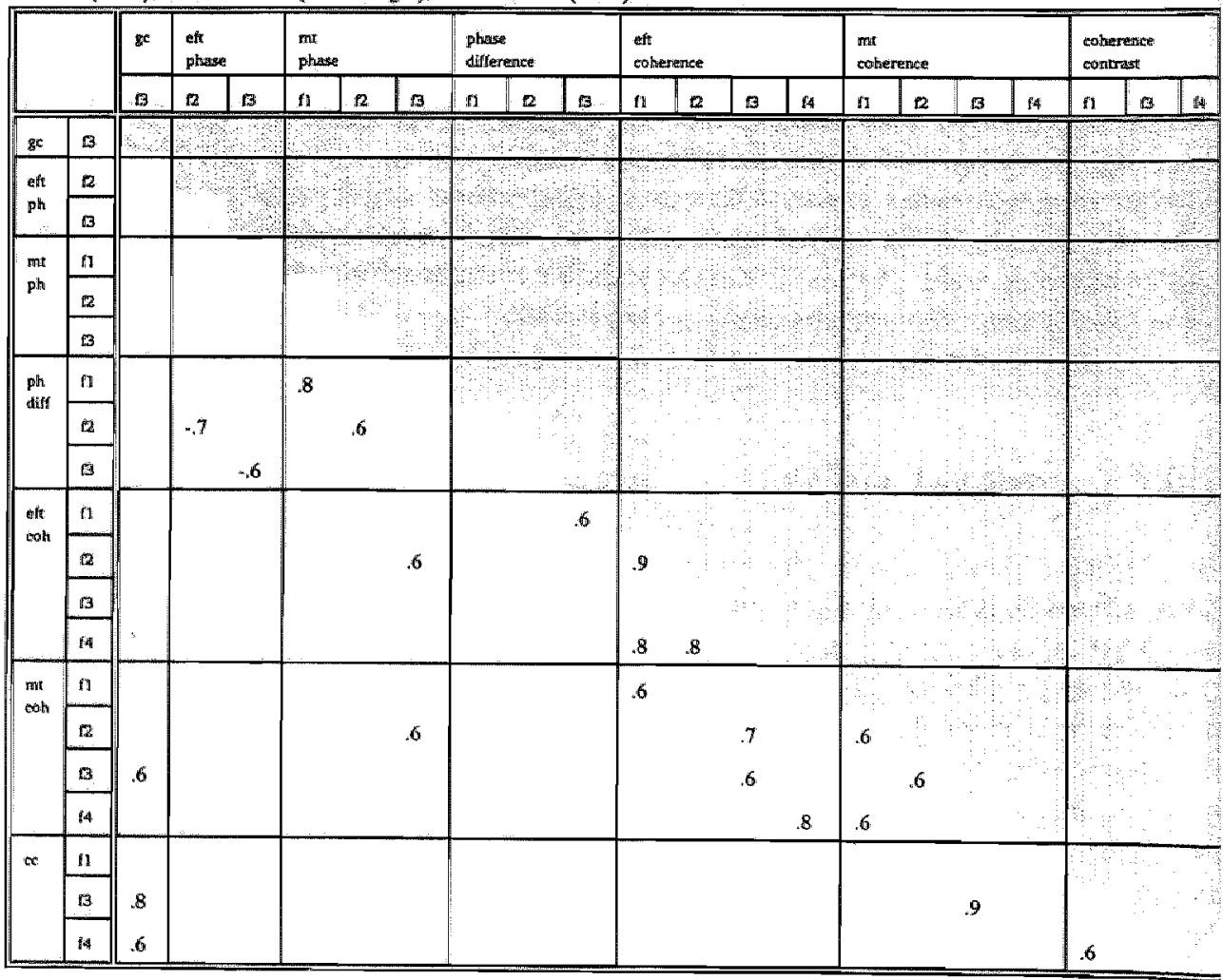


Table 11-2. Correlation Lable of standard tests parameters (only parameters having a correlation of o.55 or more are shownt). * gain when rotating to the right, $L=$ gain when rotating to the left, ADe cold irrigation of the rught ear, ASe cold ingigation of the left ear, ASx" = warm irrigation of the left ear $A D w=$ warm irrigation of we right car.

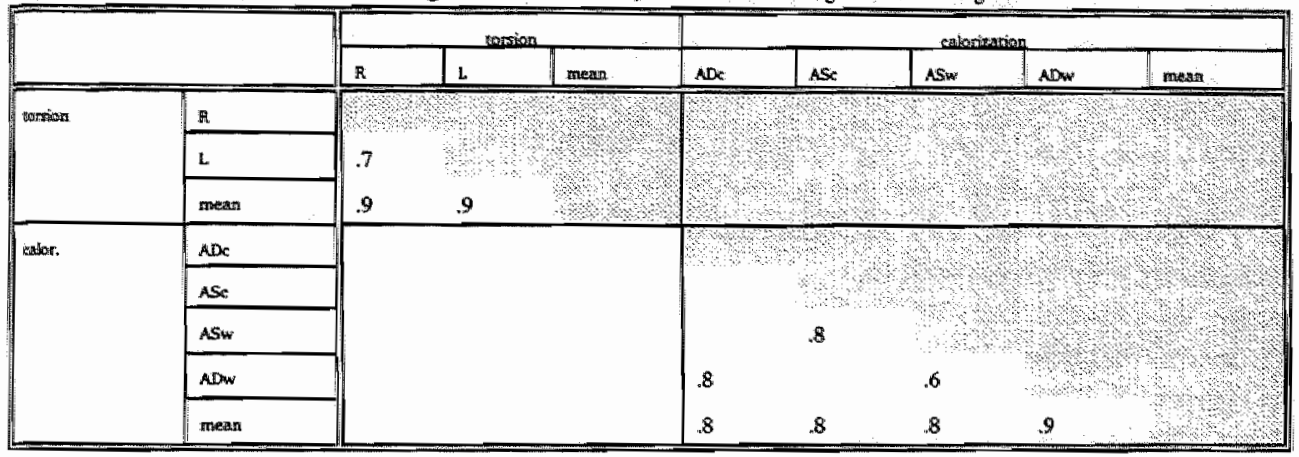

values were retained, instead of the directly observed values. Table 11-3 shows the anamnestic and the remaining test variables, used for analysis.

Discriminant analysis optimizes a linear function of the predictor variables. The parameters are chosen in such a way, that the within-group variances are minimized,

Table 11-3. Test and anamnestic variables used for discriminant analysis. eftVVIT = earth-fixed target visuo-vestibular internction test, miVVT $=$ moving target visuowestibular interaction test, $\mathrm{n} / \mathrm{p}=$ normal/pathological. $y / \mathrm{n}=\mathrm{yes} / \mathrm{no}, \mathrm{OKR}=$ aptokinetic renex, $S C Y=$ slow component velocity.

\begin{tabular}{|c|c|c|}
\hline & VVTT & standard tests \\
\hline 1 & gain contrast $\mathrm{f}=0.16 \mathrm{~Hz}$ & saccade latency $(\mathrm{m} / \mathrm{p})$ \\
\hline 2 & gain contrast $f=0.41 \mathrm{~Hz}$ & saccade velocity $(\mathrm{n} / \mathrm{p})$ \\
\hline 3 & gain contrast $\mathbb{L}=1.15 \mathrm{~Hz}$ & OKR SCV (n/p) \\
\hline 4 & mivVIT phase shif $f=0.41 \mathrm{Hx}$ & OKR SCV asymmetry (n/p) \\
\hline 5 & mIVVIT phase shif $t=1.15 \mathrm{hL}$ & torsion mean gain \\
\hline 6 & phase shif difference $\mathrm{t}=0.16 \mathrm{hiz}$ & torsion gain agymmetry \\
\hline 7 & phase shift difference $\mathbb{f}=1.15 \mathrm{H} x$ & calorization taean SCV \\
\hline 8 & eftVVTT coherence $\hat{\mathrm{t}}=0.41 \mathrm{kH}$ & calorization SCV asymmetry \\
\hline 3 & eft VVIT coherence $\mathrm{I}=0.53 \mathrm{~Hz}$ & aspecific dizziness ( $\left.y^{y / n}\right)$ \\
\hline 10 & colherence contrast $\mathrm{f}=0.41 \mathrm{HI}$ & forting feelings $(y / n)$ \\
\hline 11 & noating follings $(y / n)$ & wentigo $(y / n)$ \\
\hline 12 & vertigo $(y / n)$ & falling lendenctes $(y / n)$ \\
\hline 13 & falling tendencies $(y / n)$ & natsea $(y / n)$ \\
\hline 14 & nausea $(y / n)$ & blackouts $(y / n)$ \\
\hline
\end{tabular}


while the between-groups variance is maximized. Therefore, the interpretation of the predictor variables should have one direction (e.g, the higher the value, the more pathological the case). Asymmetry measures are made to conform to this by taking their magnitude.

The complaints "aspecific dizziness", "floating feelings", "vertigo", "falling tendencies", "nausea", and "black-outs" were entered as dichotomous variables (yes/no). Sex and age were not included, because a. MANOVA had failed to show their significance (see chapters 8,9 , and 10 ), in accordance with the literature, and $\underline{b}$. groups were not homogeneous in these respects.

Finally, to prevent the VVTT from attaining a higher score by virtue of its higher number of variables, the number of variables was limited to that of the smallest set. The variables were selected stepwise by minimizing Wilks' $\lambda$ (i.e., the proportion of the total variance in the discriminant scores not explained by differences among groups), with the F-statistic's levels for including and for removing both set to 0.5. The best 14 of the VVTT and anamnestic variables allowed correct (i.e., in accordance with the diagnoses) classification of $86 \%$ of the cases (table 11-4), while all (14) standard test and anamnestic variables did so for $72 \%$ (table 11-5). (Purely by chance, a correct classification of $16,7 \%$ is to be expected for six groups; using anamnestic variables only, $40 \%$ of the predictions was found to be correct (table 116 ). For the whiplash group, sensitivity is $80 \%$ and specificity is $81 \%$. It may seem remarkable that the whiplash patients formed the group that was best classified solely on basis of their complaints. But, of course, the whiplash syndrome seems to be a rather sensitive tool only when it is already known that all subjects are patients. If also the normal group is included, the number of overall correctly classified subjects becomes $38 \%$, and for the whiplash group the sensitivity drops to 0.30 while the specificity becomes 0.97 .)

Table 11-7 shows the results after including the normal group for analysis of the

Trable 11-4. Classification of the same data as ased to derive the discriminant function, for the best 14 of the VVTT test

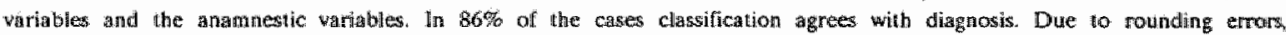
percenthanges may not sum to $100 \%$

\begin{tabular}{|c|c|c|c|c|c|c|c|c|}
\hline \multirow{2}{*}{$\begin{array}{l}\text { gchual } \\
\text { group }\end{array}$} & \multirow{2}{*}{$\begin{array}{l}\text { mumber } \\
\text { of } \\
\text { subjects }\end{array}$} & \multicolumn{7}{|c|}{ predicted goup menbership } \\
\hline & & 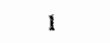 & 2 & 3 & 4 & 6 & & 7 \\
\hline 1 & 4 & $4(100 \%)$ & i & 0 & 0 & 0 & 0 & \\
\hline 2 & 8 & 0 & $7\left(8 \mathrm{~S}^{\circ} \%\right)$ & $1(13 \%)$ & 0 & 自 & 0 & \\
\hline 3 & 13 & $1(86)$ & 0 & $10(77 \%)$ & 0 & 1. $\quad(8 \%)$ & $\mathbb{1}$ & $(850)$ \\
\hline \# & 10 & 0 & $100 \%)$ & 0 & $9(90 \%)$ & 0 & 0 & \\
\hline 6 & 3 & 0 & 0 & 0 & 0 & $3(100 \%)$ & 0 & \\
\hline 7 & 4. & 0 & 1 (25\%) & 0 & 0 & 0 & 3 & $(75 \%$ \\
\hline
\end{tabular}


Tabie 11-5. Classification of the same data as used to derive the discriminan function, for all 14 of the stardard tests variables and the anaminestic variables. In $72 \%$ of the cases classification agres with diagnosis. Due to rounding enror, percentages may

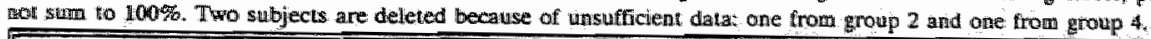

\begin{tabular}{|c|c|c|c|c|c|c|c|c|}
\hline \multirow[t]{2}{*}{ 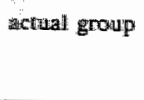 } & \multirow{2}{*}{$\begin{array}{l}\text { Dumber } \\
\text { of } \\
\text { subjects }\end{array}$} & \multicolumn{7}{|c|}{ predicted group membership } \\
\hline & & 1 & 2 & 3 & & 4 & 6 & 7 \\
\hline 1 & 4 & $2(5000)$ & 0 & $2(5090)$ & 0 & & 0 & 0 \\
\hline 2 & 7 & $1(14 \%)$ & $5(21 \%)$ & 0 & 0 & & $1(140 \%)$ & 0 \\
\hline 3 & 13 & $3(23 \%)$ & 0 & $8(62 \% 6)$ & 1 & $(8 \%)$ & $1 \quad(8 \%)$ & 0 \\
\hline 4 & 9 & $1(11 \%)$ & a & 0 & 8 & $(89 \%)$ & 0 & 0 \\
\hline 6 & 3 & 0 & 0 & 0 & 0 & & $3(100 \%)$ & 0 \\
\hline 7 & 3 & 0 & 0 & $1(33 \%)$ & 0 & & 0 & 2. $(67)$ \\
\hline
\end{tabular}

VVIT. The number of overall correctly classified subjects dropped a little from $86 \%$ to $83 \%$, and the whiplash' sensitivity and specificity remained the same ( $90 \%$ and $100 \%$, respectively), showing that the VVIT did not depend on prior knowledge that all subjects were patients.

Although the diagnoses (except for the whiplash patients) were based on the outcomes of the standard tests, surprisingly, the VVIT results allow the best classification. The lesser performance of the standard tests can not be attributed to the inclusion of the whiplash patients, for those are labeled $89 \%$ correctly. Repeating the analysis without the whiplash patients, makes the VVIT score $84 \%$, and the standard tests $70 \%$ (chance: $20 \%$ ), which are practically the same rates as with them. This would also have been expected on ground of the above-mentioned reliability of the complaints in combination with the knowledge that a subject is a patient. Perhaps the VVIT discriminates better due to the greater variation of its variables (all 10 test

Thite 116. Chassitication of the same data as wased to derive the discriminant function, for the andanestic variables only. In 40 of the cases classification agrees with diagnosis. Dute to rounding errors, parcentages may not sum 10 100\%.

\begin{tabular}{|c|c|c|c|c|c|c|c|c|c|c|c|c|c|}
\hline \multirow{2}{*}{$\begin{array}{l}\text { Bctual } \\
\text { growlig }\end{array}$} & \multirow{2}{*}{$\begin{array}{l}\text { number } \\
\text { of } \\
\text { subjects }\end{array}$} & \multicolumn{12}{|c|}{ predicted group meabership } \\
\hline & & & 1 & & 2 & & 3 & & 4 & & 6 & & 7 \\
\hline 1 & 4 & 3 & $(75 \%)$ & & $(25 \%$ & 0 & & 0 & & 0 & & 0 & \\
\hline 2 & 8 & 1 & $(13 \%)$ & & $(13 \%)$ & & $(25 \%)$ & & $(50 \%)$ & 0 & & 0 & \\
\hline 3 & 13 & 5 & $(39 \%)$ & & $(8 \%)$ & 1 & $(80 \%)$ & 1 & $(8 \%)$ & 4 & $(31 \%)$ & & $(\theta)$ \\
\hline 4 & 10 & 1 & $(10 \%)$ & & $(10 \%)$ & 0 & & & $(80 \%(6)$ & 0 & & 0 & \\
\hline 6 & 3 & 0 & & 0 & & & $(33 \%)$ & 0 & & & $(67 \%)$ & 0 & \\
\hline 7 & 4 & 1 & $(25 \%)$ & 0 & & 0 & & & $(25 \%)$ & 0 & & & $(50 \%)$ \\
\hline
\end{tabular}




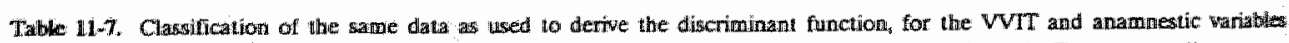
for six gromps of patients and a normal groug. In $83 \%$ of the cases classification agrees with diagnotis. Due to rounding encus percentage may not sum to $100 \%$.

\begin{tabular}{|c|c|c|c|c|c|c|c|c|c|c|c|c|c|}
\hline \multirow{2}{*}{$\begin{array}{l}\text { actual } \\
\text { group } \\
\text { number }\end{array}$} & \multirow{2}{*}{$\begin{array}{l}\text { number } \\
\text { of } \\
\text { subjects }\end{array}$} & \multicolumn{12}{|c|}{ predicted group menbership } \\
\hline & & 0 & 1 & & 2 & & 3 & & 4 & & 6 & & 7 \\
\hline 0 & 6 & $6(100 \%)$ & 0 & 0 & & 0 & & 0 & & 0 & & 0 & \\
\hline 1 & 4 & 0 & $4(100 \%)$ & 0 & & 0 & & 0 & & 0 & & 0 & \\
\hline 2 & 8 & $1(13 \%)$ & 0 & 6 & $(7506)$ & 1 & $(13 \%)$ & 0 & & 0 & & 0 & \\
\hline 3 & 13 & 0 & $1(8 \%)$ & 0 & & 10 & $(77 \%)$ & 0 & & 1 & $(8 \%)$ & 1 & $(8 \% 5)$ \\
\hline 4. & 10 & 0 & 0 & 1. & $(10 \%)$ & 0 & & 9 & $(90 \%)$ & 0 & & 0 & \\
\hline 6 & 3 & 0 & 0 & $a$ & & 1 & $(33 \%)$ & 0 & & 2 & $(67 \%)$ & 0 & \\
\hline 7 & 4 & $1(25 \%)$ & 0 & 0 & & 0 & & 0 & & 0 & & 3 & $(75 \%)$ \\
\hline
\end{tabular}

variables were continuous for the VVIT, while only four were so for the standard tests), which can be checked by analyzing with the original standard test values instead of normal/pathological indications. Another possibility is, that replacing missing or removed values by the group average gave the VVIT an advantage. Compared to leaving out those cases, replacing diminishes the within-group variance, and thus might enable a better classification. More VVIT values were replaced by group averages than standard test values. Therefore, the analyses were repeated with the pruned data. Without the normals, $88 \%$ was classified correctly by using the 14 best variables, and $82 \%$ when the normals were included, which are practically the same rates as for the data with replacements.

It is concluded that the VVIT probably is at least as good as the standard tests in allowing diagnosing type of pathology.

Using the ordering of the variables as defined by the stepwise inclusion, mtVVIT phase shift at $0.41 \mathrm{~Hz}$ and $1.15 \mathrm{~Hz}$, vertigo complaints and gain contrast at $0.16 \mathrm{~Hz}$ and $1.15 \mathrm{~Hz}$ seem to be the most important variables for the VVIT, and asymmetry and mean of calorization, saccade velocity, and complaints of vertigo and falling, for the standard tests. 


\section{Discriminating power of the Visuo-Vestibular Interaction Test for side of pathology, compared to that of calorization.}

\subsection{Abstract.}

In previous chapters (5 .. 11) a vestibular function test (named Visuo-Vestibular Interaction Test: VVIT) was presented, in which a visual target may move asynchronously with a rotatory chair. The various forms of sensitivity and specificity were there discussed, dealing with how well the VVIT discriminates patients from normals and from group of patients from another. The current chapter investigates whether VVTT results allow recognition of patients having unilateral vestibular dysfunctions, and if so, whether side localization agrees with calorization results.

Using a novel filter technique called "stimulus-ordered median filtering", distinct gain patterns were found, but we could not reconcile these with the caloric data. Contrary to the low-frequency oscillation in the dark, it appears to be unnecessary to select first on phase shift in order to separate clinically relevant large gain asymmetries from clinically irrelevant ones.

\subsection{Introduction.}

A full introduction of the VVIT was given earlier (chapter 5: "Effect of asynchronous visual stimulation on the Vestibulo-Ocular Reflex gain of normals"), so here only the essentials will be repeated.

In order to elude the variation inherent to measuring the vestibulo-ocular reflex (VOR) in the dark, a visual target is presented. The usual ways in which the resulting visuo-vestibular interaction (VVI) is studied, (namely, by offering either an earth-fixed target or a chair-fixed target) allow, but may not require, the two systems to interact. To force an interaction between smooth pursuit (SP) and VOR, we use a visual target which moves asynchronously with the chair. Predictive effects are reduced by having the chair move in a pseudo random (sum-of-sines) way. As both visual target movement and chair movement are below the SP upper limit, we expect normals and patients to perform equally well to either one of them. But because the combination is above the SP upper limit, we expect patients to perform worse than normals to the combined stimulus, assuming that they will overburden their SP system in an effort to compensate for the insufficient vestibular function. By subtracting the visuo-vestibular interaction with an earth-fixed target (eftVVI; i.e, visually enhanced VOR) stimulus and the response to it, from the visuo-vestibular interaction with an asynchronously moving target (mtVVI) stimulus and the response to it, the visual components are obtained. It is hypothesized that the vestibular function is reflected by the ratio of the 
smooth and saccadic eye movements (and thus by the gain of the median-filtered response). Different phase shifts for normals and patients and for eftVVI and $\mathrm{mtVVI}$ might be expected in relation to the complexity of the stimulus and the type of neural processing it is subjected to.

\subsection{Material and methods. \\ 12.3.1 Subjects.}

Data were obtained from the subjects who were described earlier (see chapter 8 ). In short, 42 patients (median age: 45) and 6 controls (median age: 30) were examined. The patients felt in 6 categories: those having initial stages of Meniere's syndrome (4), those having peripheral vestibular dysfunctions, centrally well compensated (8) and centrally partly compensated (13), whiplash victims (10), BPPD patients (3), and those for which the causes of their complaints are unknown (4). They were diagnosed by an experienced vestibulologist (dr. Kingma), using anamneses and results of standard tests (see below). All subjects were tested without optical corrections.

\subsubsection{Experimental set-up.}

The experimental set-up was as reported previously (chapter 5), and so only a brief description follows here. Subjects sat in a rotary chair moving in a pseudo random way (sum-of-sines: $0.16,0.41$, and $1.15 \mathrm{~Hz}$, each with a maximum velocity of $10 \% \mathrm{~s}$ ), while watching a dot which was either earth-fixed or moving independently $(0.53 \mathrm{~Hz}$, with a maximum velocity of $32^{\circ} / \mathrm{s}$ ). The eye position was measured using infra red reflection, and the head velocity was monitored with an angular rate sensor. Eye position, head velocity, and visual target position were sampled at a $50 \mathrm{~Hz}$ frequency for 125 seconds by a 12 bit analog-to-digital ( $A D$ ) converter (the first two after being filtered analogously with a cut-off frequency of $10 \mathrm{~Hz}$ ) and were digitally stored for offline analysis.

Standard tests consisted of calorization $\left(30^{\circ}\right.$ Celsius and $44^{\circ}$ Celsius, in the order right cold, left cold, left warm, right warm), torsion in the dark $(0.1 \mathrm{~Hz}$ frequency, 50 - Is peak velocity), a smooth pursuit test $\left(0.29 \mathrm{~Hz}\right.$ frequency, $15^{\circ}$ amplitude), and an optokinetic test $(10,20,30$, and $40 \% / \mathrm{s}$ velocity, both horair and anti-horair). Left and right horizontal eye position was measured by electro-oculography, and was sampled at $100 \mathrm{~Hz}$. 


\subsubsection{Digital signal analysis.}

Memory limitations restricted the number of samples to 2048 , equivalent to 41 seconds of testing. And so only the first part of the total recording was used.

Position data were digitally differentiated to velocities, after which the total stimulus was calculated by subtracting the head movement from visual target movement. Fast components and eye blink artefacts were removed or attenuated by a median filter with a width of $0.15 \mathrm{~s}$. The resulting data were ordered according to the stimulus values (thus scrambling time), and were median filtered with a width of 25 samples (see section 4.2.4 "Stimulus-ordered median filter" of chapter 4 "Slow component characterization"). After restoring the chronological order, the filtered data were plotted as line graphs, showing filtered response velocity versus stimulus velocity.

\subsubsection{Statistical analysis.}

Statistical analysis comprised of regression techniques. The ratio of the gains (as given by the angles of the lines fitted separately to the leftward and the rightward movements) was compared for the various groups. Because the angle of a regression line depends on the gain, as well as on randomness, and because only the former influence is wanted, the average gain of the regressions of response on stimulus values $(y \rightarrow x)$ and of stimulus on response values $(x \rightarrow y)$ was taken (thereby canceling the angle-changing effect of scatter). Furthermore, both possible types of linear regression (i.e., allowing a variable y-axis intercept, and forcing the line through the origin) was tried. So in total, eight regression fits were done per subject: 2 (left-, rightward) $\times 2(y \rightarrow x, x \rightarrow y) \times 2$ (y-intercept, through origin).

\subsection{Results and discussion.}

To assess gain asymmetry, the response velocity is plotted in the usual way against the stimulus velocity (figure 1).

For single-sine stimuli, the ideal figure is an ellipse, of which the tangent of the main axis" angle gives the gain, and the width at zero stimulus velocity is twice the product of gain, stimulus amplitude, and the sine of the phase shift (see section 4.2.4 "Stimwlus-ordered median filter" of chapter 4 "Slow component characterization").

For sum-of-sines stimuli, however, interpretation is less direct, because the various frequencies may have different gains and different phase shifts. Different gains result 


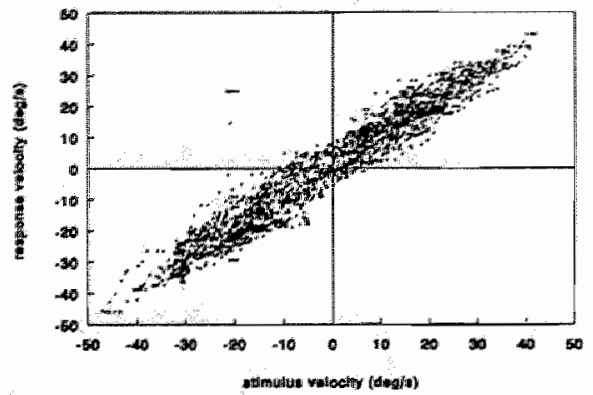

117ig 12-1. Scatter diagram of the stow component ( $\mathrm{SC}$ ) of a normal response $10 \mathrm{miVVI}$ stimulition. The outlers in the upper left quadiant result from an incompletely remowed eye blink artefact:

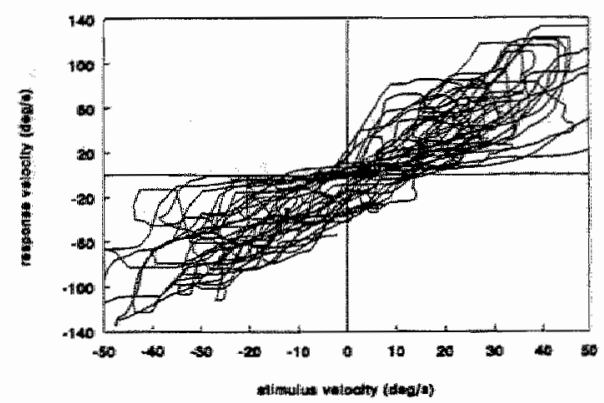

Fig 12-2 Line diagran of a patient's response $\mathrm{SC}$ (calonic response left weaker than right, torsion in the dark wormall) to miVVl stibmilation. "Loops" result from changing peali welocities and from different gains for the various freguertcies.

in widening of the ellipsoid scatter diagram (figure 2, here given as a line graph to emphasize the "loops"), which may be hard to distinguish from phase-induced widening. Therefore, the asymmetry of only the gain is discussed, and this is a frequency-averaged asymmetry.

The total stimulus (i.e, the apparent velocity of the visual target) was calculated by subtracting the head velocity from the actual velocity of the visual target. Using positive numbers to indicate rightward eye movements, this means that the left panels of the figures in this chapter contain head movements to the right.

Due to the "push-pull" configuration (i.e., bilateral commissural inhibition") in the vestibular nuclei of a pair of synergistic semicircular canals (Shimazu \& Precht, 1966; Louie \& Kimm, 1976), it can be expected that the asymmetry caused by a dysfunction of one of the vestibular end organs is much reduced, while a one-sided lesion more central than the vestibular nuclei may display its full asymmetry. During calorization, in contrast, end organ asymmetries show up far less reduced, because only one side at

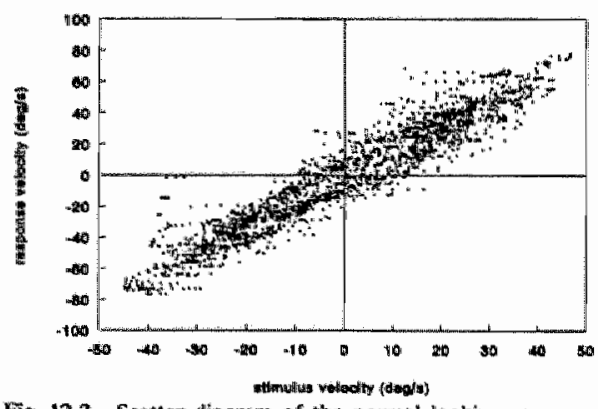

Fig 12-3. Scatter diagram of the nomal looking response of a patient (unilateral dys(unction, right weaker than left).

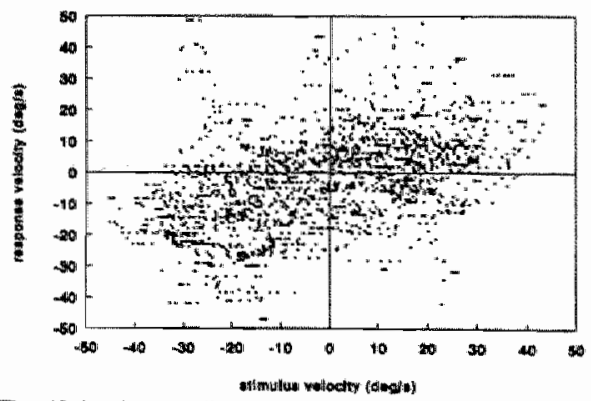

Fig- 12-4. Scalner diagram of inconsistent rexponses of a patient (no diagnosis reached by standard tests).

\footnotetext{
Becatse of the fining frequency in the resting state, which turns an essentially unidirectional sensor into a bidirectional one, "untuibition" is here to be interpretted as "stimulation of the alliemative response".
} 
a time is stimulated. So at the one hand, VVI asymmetries may be expected to be much less dramatic than calorization asymmetries, but at the other hand, results may show less random variation because no uncertainties like ear duct anatomy and heat transfer influence performance, and because stimulation is done at more physiological frequencies. The asymmetry in directional sensitivity per canal (second law of Ewald) makes, in combination with the above-mentioned push-pull configuration, that a weaker vestibular response at one side will result in a lower overall gain for the ipsilateral rotation than for the contralateral one (see appendix). Examples of patients' data are given in figures $12-2,12-3$, and $12-4$. Different velocity ranges reflect cailbrational problems and are not considered. Instead, attention is paid to the overall shape, and especially to any left-right asymmetry. Apart from some occasional gain asymmetries, no systematic deviations are obvious from visual inspection of the scatter diagrams of four groups of patients (unilateral dysfunction with left as the weakest side, idem right, and both being centrally compensated or uncompensated) and a normal group. But most patients produced more or less symmetrical graphs somewhere between the extremely narrow one in figure 12-3 and the extremely wide one in figure 12-4. No relation between width and diagnosis was detected.

The ratio of the gains (as given by the angles of the lines fitted by linear regression separately to the leftward and the rightward movements) was compared for the various groups. To diminish the influence of scatter on the slopes of the fitted lines, the average was calculated from $(y \rightarrow x)$ and $(x \rightarrow y)$ regressions. Furthermore, both possible types of linear regression (i.e., allowing a variable $y$-axis intercept, and forcing the line through the origin) was tried. Although some scatter diagrams have their regression lines not passing through the origin, results of both types of regression were not qualitatively different as can be read from table 12-1. The geometric means are here tabulated, because these restore the symmetry that has been lost by taking ratio $\mathrm{s}^{2}$. The essence of these resuits are summarized in table 12-2, which should be compared with the expectations in table 12-3. The results are incompatible with what was anticipated.

Two points deserve attention. First, Hamid (1990) has shown for (low frequency) dVOR responses, that gain asymmetry only corresponds to the side of lesion, when the phase is abnormal. And second, fitted lines did not always agree with visual impressions, probably because linear regression by the least squares method is very sensitive to outliers. As shown in section 3.3 "Calibration" of chapter 3 "Material and methods", regression based on minimizing the mean absolute error produces lines more consistent with the bulk of the data than one based on minimizing the root

2. Clearly. a leftward gain twice as high as the rightward gain is symmetrical whth the rightward being twice as strong as the leftword. But their natio's 0.5 and 2.0 are not symmetrical around 1.0 (gains to the right and to the left equally hight). Taking logarithms restlores that symmetry, resulting in geometric means. 


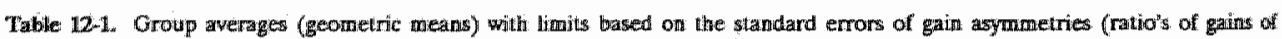

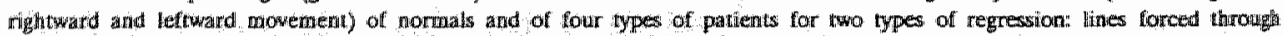

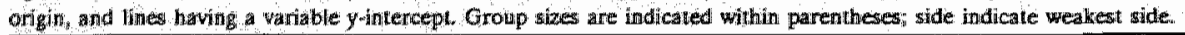

\begin{tabular}{|c|c|c|c|c|c|}
\hline \multirow{2}{*}{$\begin{array}{l}\text { type of } \\
\text { regression }\end{array}$} & \multicolumn{5}{|c|}{ group } \\
\hline & $\begin{array}{l}\text { normalls } \\
\text { (6) }\end{array}$ & $\begin{array}{l}\text { unilateral dysfurc } \\
\text { tion, left uncont } \\
\text { penasated (i) }\end{array}$ & $\begin{array}{l}\text { unilateral dysfunc- } \\
\text { tion, lleft, compen:- } \\
\text { salled (4) }\end{array}$ & $\begin{array}{l}\text { unilateral dysfunc- } \\
\text { tion, right, uncom- } \\
\text { pensated }(7)\end{array}$ & $\begin{array}{l}\text { urmilateral tys- } \\
\text { function, thight, } \\
\text { compensated (3) }\end{array}$ \\
\hline $\begin{array}{l}\text { through } \\
\text { tritgln }\end{array}$ & $\begin{array}{c}0.96 \\
{[0.91 .1 .01]}\end{array}$ & 0.53 & $\begin{array}{c}0.88 \\
{[0.80 .0 .981]}\end{array}$ & $\begin{array}{c}0.89 \\
{[0.85 . .0 .93]}\end{array}$ & $\begin{array}{c}0.60 \\
{[0.47 \ldots 0.76]}\end{array}$ \\
\hline $\begin{array}{l}\text { with } \\
\text { yintercept }\end{array}$ & $\begin{array}{c}1.02 \\
0.98 .1 .05\end{array}$ & 0.37 & $\begin{array}{c}0.83 \\
{[0.70 .0 .98]}\end{array}$ & $\begin{array}{c}0.91 \\
{[0.85 \ldots .0 .98]}\end{array}$ & $\begin{array}{c}0.45 \\
{[0.29 .0 .79)}\end{array}$ \\
\hline
\end{tabular}

mean square error. Alternatively, one may remove outliers better than was done now. The current detection and removal by median filtering the time series was a quick, approximate method, suitable for subsequent frequency analysis by Fourier techniques. Better removal may actually use the present graphs, as explained in section 4.2.4 "Stimulus-ordered median filter" of chapter 4 "Slow component characterization".

So the data was reexamined, taking only cases with deviant phase shifts, and regressing on stimulus-dependent median filtered data instead of on only time series median filtered data.

Using the frequency-specific lower and upper limits for phase shifts as found in chapter 6 "Effect of asynchronous visual stimulation on the Vestibulo-Ocular Reflex phase shift of normals" (and repeated here in an uncorrected form in table 12-4), and excluding outliers as given in table 9-2 of chapter 9 "Effect of asynchronous visual stimulation on the Vestibulo-Ocular Reflex phase shift of patients, compared to that of normals", patients were selected who deviated for all three chair-delivered frequencies. Only one subject satisfied this criterium. And indeed, she did have a severe right/left asymmetry. But contrary to her diagnosis "unilateral dysfunction; right weaker than left (centrally well compensated)", her VVI gain was for head move-

Tabe 12-2 VVIT gain asymmetries found in patients. $L=$ gain for leftwand chair rotation, $\mathrm{R}=\mathrm{gmin}$ for rightward chair rotation, ${ }_{4}$ uncompensated, + compensated. Comparte this with the expectations given in table 12-3.

\begin{tabular}{|c|c|c|c|}
\hline & & \multicolumn{2}{|c|}{ compensated } \\
\hline & & - & 4 \\
\hline \multirow{2}{*}{$\begin{array}{l}\text { weathest } \\
\text { sidde }\end{array}$} & left & \multirow{2}{*}{$\begin{array}{l}\mathrm{R}: \mathbb{L} \\
\mathrm{R} \leq \mathbb{L}\end{array}$} & \multirow{2}{*}{$\begin{array}{l}R \leq \mathbb{L} \\
R \propto L\end{array}$} \\
\hline & right & & \\
\hline
\end{tabular}

Table 12-3. Expected VVIT gain asymmetries for patrerits having one-sided dysfunctions. $\mathrm{L}=$ gain for lefitward thair

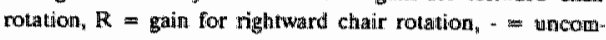
pensaned, $+=$ compensated.

\begin{tabular}{|c|c|c|c|}
\hline & & \multicolumn{2}{|c|}{ compensatied } \\
\hline & & $\approx$ & + \\
\hline \multirow{2}{*}{$\begin{array}{l}\text { weakest } \\
\text { side }\end{array}$} & left & \multirow{2}{*}{$\begin{array}{l}R \approx \mathbb{L} \\
R \ll \mathbb{L}\end{array}$} & \multirow{2}{*}{$\begin{array}{l}\mathrm{R} \geq \mathrm{L} \\
\mathrm{R} \leq \mathrm{L}\end{array}$} \\
\hline & right & & \\
\hline
\end{tabular}




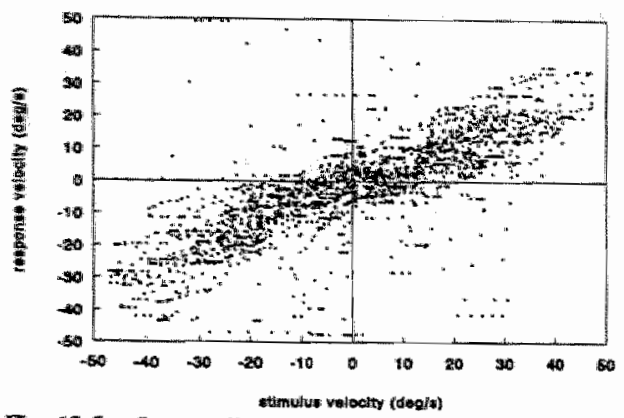

Fig 125. Scatter diagram of the responses of the only phatitunt (right weaker that left) baving abnonal phase shifts lor all three chair-dielivered frequencies.

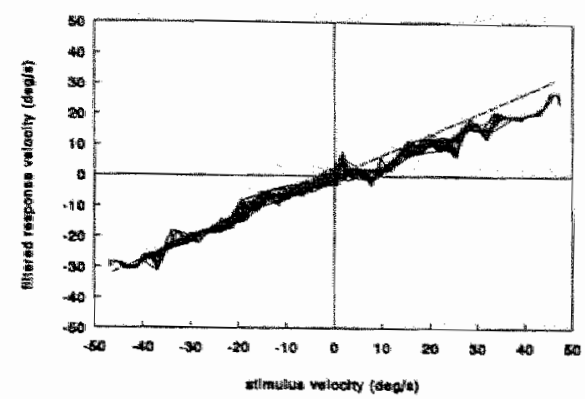

Fig. 12-6. Line diagram of the data of tigure 12-5, after median filtering the stimulus-ordered responses. Note that the gain when the head is moved to the left (righ panel) is clearly less than when mowing to the right (left panel).

ments to the left lower than for those to the right (right/left ratio is 1.20 ). Figure 12-5 gives the scatier diagram of the slow component velocities (with several incompletely removed fast components) versus the stimulus velocity. Figure 12-6 gives the line diagram of the results of median filtering the stimulus-ordered response velocities, with the regression line of the rightward head velocities indicated and extrapolated to the leftward velocities (remember that the leftward head velocities are in the right panel and vice versa). In this latter figure, the lower gain of the head movements to the left can be seen clearly.

Because the normative phase shift data was obtained by a small group of only six normals, the derived normal range may be too conservative (Royston \& Matthews, 1991). Furthermore, the limits were set considering univariate probabilities separately. But our inclusion criterium is "being outside the normal ranges for three frequencies simultaneously", and so some contraction of the univariate ranges will not make the

Talbie 12-4. Lower and upper limits of the three ranges used 10 define normal phase shifts for mivVlT stimulalton. The full ramges are based on uncorrected phase shifts of six normals; the $75 \%$ and $50 \%$ ranges are derived from these.

\begin{tabular}{|c|c|c|c|c|}
\hline \multirow{2}{*}{$\begin{array}{l}\text { morrilal mith } \\
\text { phase sinifl ramge }\end{array}$} & \multicolumn{3}{|c|}{ chair-delliwered "stimulus frequency } & \multirow[b]{2}{*}{ fimit } \\
\hline & $0.16 \mathrm{H} x$ & $0.41 \mathrm{HZZ}$ & $1.15 \mathrm{~Hz}$ & \\
\hline \multirow[t]{2}{*}{ full } & -15 & -2.5 & -10.5 & lower \\
\hline & 10.5 & 18.0 & 35.0 & lippens \\
\hline \multirow[t]{2}{*}{$75 \%$} & 0.0 & 0.1 & -48 & lower \\
\hline & 9.9 & 15.4 & 294 & upper \\
\hline \multirow[t]{2}{*}{$50 \%$} & 1.5 & 27 & 1.0 & Lower \\
\hline & 7.5 & 12.9 & 23.7 & upper \\
\hline
\end{tabular}




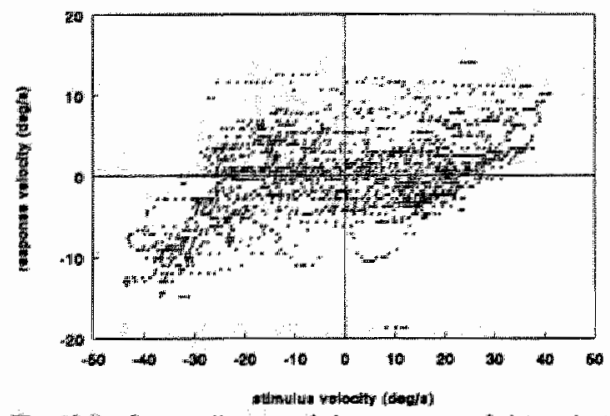

Fig 12-7. Sicatter diagram of the responses of the patient (trigh wealker thein left) hawing second severest phase shifts.

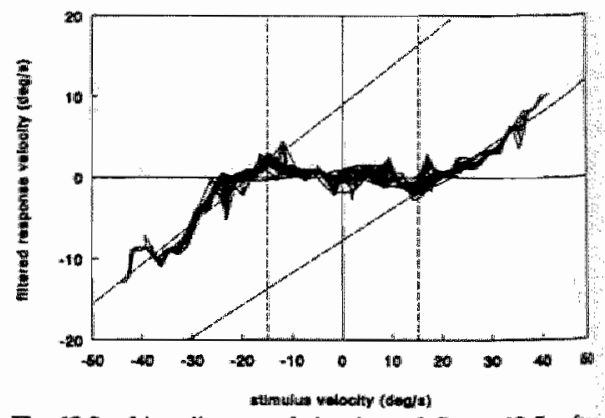

Fig 128. Line diagram of the data of figure 12-7, after mientian filtering the stimullus-ordered mesponses. Note symmetrical threshold of $\pm 15 \% / s$ and the asymmetnical gains.

probability of a type I error exceed the 5\%. For this occasion, the normal ranges were contracted to $75 \%$ of their original sizes, giving the thresholds mentioned in table 12-4. This resulted in finding one additional subject. Her responses are given in figures $12-7$ and 12-8. The former is the scatter diagram of the slow component velocities (with some incompletely removed fast components) versus the stimulus velocity, and the latter is a line diagram of the results of median filtering the stimulus-ordered data. At lower stimulus velocities the subject responded not systematically (inferred from the fact that the stimulus-dependent median filter results are near zero; the slow component velocities show the eye wandered around random. $\left.1 y^{3}\right)$. Only at higher velocities the subject responded more consistently, but with a constant, direction-dependent velocity offset. This suggests a vestibular threshold of 15 \%/s. To assess the gain asymmetry, regression was only done on data outside the flai $\left[\begin{array}{ll}-15 & . .15\end{array} \% / \mathrm{s}\right.$ stimulus velocity range. Again a strong right/left asymmetry was found

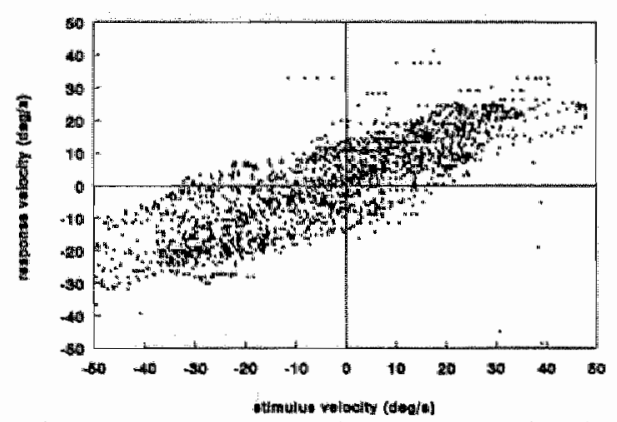

12-9. Seatter diagram of the patient (whiplash) having third severest phase shifits.

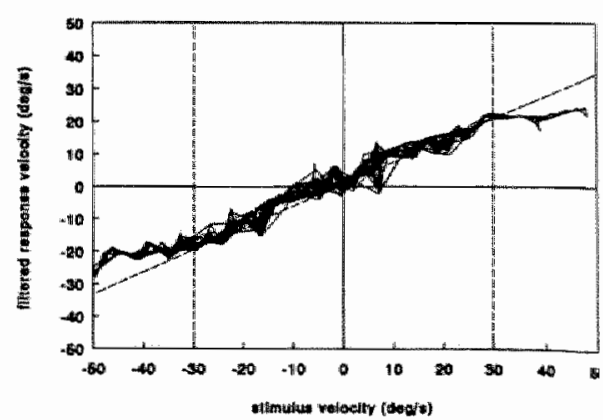

Wug. 12-10. Line diugram of the data of figure 12-9, afu medizn filtering the stimulus-ordered responsesi Note th symmetrical saturation byond $\pm 30 \% /$ s.

\footnotetext{
3a Radowly with respect to the sum-of-4sines stimulus; perhaps the subject responded for the lower velocities to a subset (e; the chiait movement onity)
} 
(1.54), and again it was opposite to the one found by calorization.

Further reduction of the normal phase shift ranges to $50 \%$ of their original sizes, resulted in another additional case. This patient was not diagnosed as having anilateral dysfunction, however, but as having whiplash trauma. Her responses are given in figures $12-9$ and $12-10$. The former is the scatter diagram of the slow component velocities (with some incompletely removed fast components) versus the stimulus velocity, and the latter is a line diagram of the results of median filtering the stimulus-ordered data. Outside the $[-30 . .30] \% / s$ stimulus velocity range, saturation accurs, suggesting that she relies heavily on her smooth pursuit system. This might indicate bilateral vestibular hypofunction or insufficient integration of visual and vestibular information. Given the anamnesis (i.c., having been involved in a whiplashproducing car accident), the latter possibility seems to be the most probable. Because of the saturation, regression has been done on the data within the $\left[\begin{array}{lll}-30 & 30] & \%\end{array}\right.$ range only. A very mild right/left asymmetry was found: 1.08 .

Another way of increasing the number of subjects eligible for analysis of asymmetry, is by relaxing the requirement of deviating for all three chair-delivered frequencies, to having an abnormal phase shift for at least two out of the three frequencies. This resulted in finding one patient having Meniere ( $25 \%$ of that group), three unilateral patients, well compensated (38\%), one whiplash patient (11\%), and two patients from the rest groups with each less than four members $(22 \%)$. With none of the tried relaxations, more than $15 \%$ of all unilateral dysfunctional patients (both compensated and uncompensated) could be incorporated by phase shift criteria. In chapter 11 "Discriminating power of the Visuo-Vestibular Interaction Test for type of pathology, compared to those of standard tests", it was shown that a discriminant function for standard tests results and anamneses could recognize $76 \%$ of them, but using such a criterium is no solution because Hamid's condition for assessing gain asymmetry (namely, excessive phase shifts) is then violated. In his publication, Hamid found $63 \%$ of his caloric patients had a deviant phase shift (inferred from counting the numbers of caloric deviant cases in panels $\mathrm{A}$ and $\mathrm{B}$ from his figure 1). This difference might be explained by the VVIT being done at higher frequencies, and/or by the influence of the visual information on the phase shift of responses to chair-delivered frequencies.

As a third approach, it was checked whether abnormal phase shifts are necessary at all in case of VVI (as they do appear to be in case of the dVOR). Using diagnoses, the most clear unilateral patients were chosen (excluding, e.g, patients having "unilateral labyrinthine dysfunction with left weaker than right, centrally overcompensated, possibly an early stage of Meniere"). The responses of the control group were used to define a normal range. In doing so, it is implicitly assumed that neither phase shift nor any other variable correlates with a factor influencing the asymmetry of VVI 


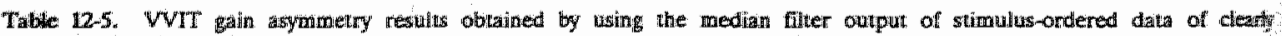

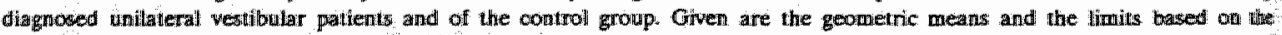
standard errors (not 10 be confitised with the $95 \%$ referemoe ranges as menioned in the text)

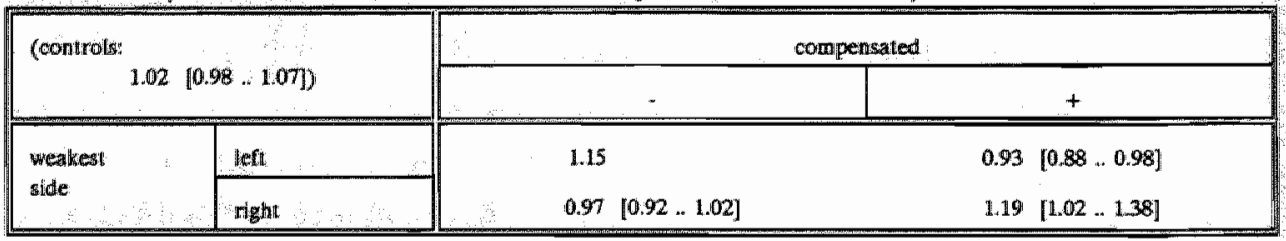

gains when rotating to the left or to the right. The geometric mean for the normals is 1.02 , and the $95 \%$ standard reference range is $[0.77 \ldots 1.36]$. Only one patient was found to have an asymmetry outside the normal range, and that is the same one who appeared when the normal phase shift range was contracted to $75 \%$ of its original size (figures $12-7$ and 12-8). She was having a VVI asymmetry opposite to the caloric one. Of the two borderline cases, one had a VVI asymmetry conform the caloric one, and the other opposite to it.

The average results are given in table 12-5. Again, these are incompatible with the expectations as given in table 12-3.

The most severe phase shifts occur with the patients having the most deviating graphs when plotting the median filter output of the stimulus-ordered response velocities versus the stimulus velocities. The right/left asymmetry from figure $12-6$, the thresholds from figure $12-8$ and the saturation of figure $12-10$, are not or only to a much lesser extent found in the remaining unilateral patients. So, contrary to the lowfrequency dVOR, it is not necessary to select first on phase shift in order to separate the clinically relevant large gain asymmetries from the clinically irrelevant ones.

Based on the three above-mentioned approaches ( $\underline{1}$. analyzing scatter diagrams with linear regression, 2. selecting by phase shift and analyzing filltered data, and $\underline{3}$. defining normal ranges of asymmetry for filtered data), it is concluded that VVI seems to give interesting information on gain asymmetry, but that we did not succeed in reconciling it with caloric data.

\subsection{Appendix}

The semicircular canals (SCCs) have asymmetric responses. The horizontal SCCs have higher gains for ipsilateral rotation (rotation to the same side as where the organ resides) than for contralateral rotation, and by subtracting the response of the 
a

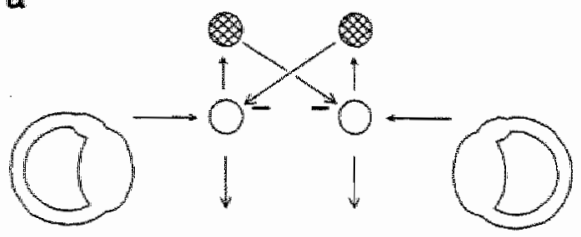

b

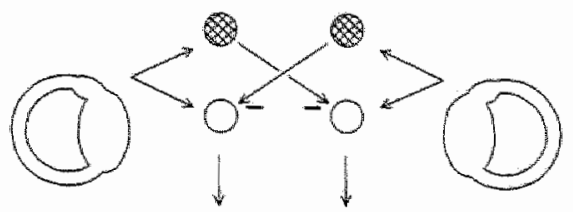

Fig 12-11. Two possible pusti-pull configurations (hatching indicates inlibiting neurones). ln a the information trom the ip. siliateral semicircular canal might be amplified by positive feedback (crossing the commissure nutce may result in de-inhabition), whith in only commissural inhibition without feedback can occur.

other canal, the final output of each canal is made less asymmetric (Louie \& Kimm, 1976). Figure 12-11 shows two possible configurations. The one given under $a$ may allow positive feedback: the signal from one side is inverted and crosses the commissure, is at the other side inverted again and returns to its original side as a positive signal. The configuration given under $b$ does not allow returning of the signal to its original side and so no feedback is possible here. Although the different schemes obviously have different final gains, the final gain asymmetry is the same.

Assuming a response table as given in table 12-6, and assuming a transfer factor $k$ for the inhibitive path, the output, the gains, and the right/left gain ratio for a clockwise (CW) rotation $d$ and a counter-clockwise (CCW) rotation $-d$ for the configuration of figure $12-11$ a can be written as:

$$
\begin{array}{ll}
C W:\left[\begin{array}{l}
\text { out }_{L}=-b \cdot c-k \cdot \text { out }_{R} \\
\text { out }_{R}=a-k \cdot \text { out }_{L}
\end{array}\right. & \text { out }_{R}-\text { out }_{L}=\frac{a+b \cdot c}{1-k} \\
\operatorname{gain}_{C W}=\frac{a+b \cdot c}{d \cdot(1-k)} & \text { out }_{R}-\text { out }_{L}=-\frac{a \cdot c+b}{1-k} \\
\text { CCW: }\left[\begin{array}{l}
\text { out }_{L}=a \cdot c-k \cdot \text { out }_{R} \\
\text { out }_{R}=-b-k \cdot \text { out }_{L}
\end{array}\right. & \operatorname{gain}_{C C W}=\frac{a \cdot c+b}{d \cdot(1-k)} \\
\frac{\operatorname{gain}_{C W}}{\operatorname{gain}_{C C W}}=\frac{a+b \cdot c}{a \cdot c+b} &
\end{array}
$$




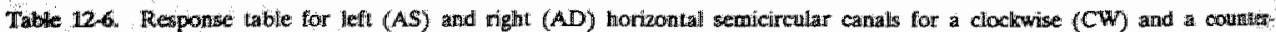

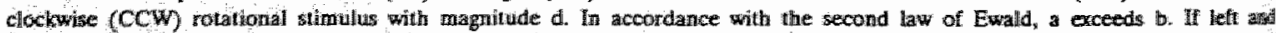
right organs response edually weil, $c$ is unity, if left responses less than righ, then $c$ is less than wnity, and otherwise it is greatur inati mity.

\begin{tabular}{|c|c|c|c|c|}
\hline \multirow{2}{*}{\multicolumn{2}{|c|}{$\begin{array}{r}1 \\
\end{array}$}} & \multicolumn{2}{|c|}{ side } & \multirow{4}{*}{$\begin{array}{l}a>b \\
\mathrm{AS}<\mathrm{AD}: c<1 \\
\mathrm{AS}>\mathrm{AD} c>1\end{array}$} \\
\hline & & AS & $A D$ & \\
\hline \multirow[t]{2}{*}{ rotation } & CW & \multirow[t]{2}{*}{$. b . c$} & a & \\
\hline & cew & & $-b$ & \\
\hline
\end{tabular}

$C W:\left[\begin{array}{l}\text { out }_{L}=-b \cdot c-k \cdot a \\ \text { out }_{R}=a-k \cdot b \cdot c\end{array}\right.$

$$
\begin{aligned}
& \operatorname{out}_{R}-\operatorname{out}_{L}=(a+b \cdot c) \cdot(1-k) \\
& \operatorname{gain}_{C F}=\frac{(a+b \cdot c) \cdot(1+k)}{d} \\
& \operatorname{out}_{R}-\operatorname{out}_{L}=-(a \cdot c+b) \cdot(1+k) \\
& \operatorname{gain}_{C C W}=\frac{(a \cdot c+b) \cdot(1+k)}{d}
\end{aligned}
$$$$
C C W:\left[\begin{array}{l}
\text { out }_{L}=a \cdot c-k \cdot b \\
\text { out }_{R}=-b-k \cdot a \cdot c
\end{array}-\right.
$$

$$
\frac{\operatorname{sain}_{C H}}{\operatorname{gain}_{\mathrm{CCW}}}=\frac{a+b \cdot c}{a \cdot c+b}
$$

The SCC's response asymmetry stems from the fact that the firing frequency in rest is about 110 action potentials per second, while the maximum is not simply 220 , but circa 400 (Fernández \& Goldberg, 1971; Goldberg \& Fernández, 1971a, 1971b). Assuming that this asymmetry expresses itself completely in the gains (and not in the saturation limits), we may substitute $a=3.6 \cdot b$. In case of the left SCC's gain being halved, $c=0.5$, and an overall asymmetry of 1.46 can be expected. 


\section{General discussion.}

\subsection{Detrimental factors.}

Several factors can influence the VOR, and thus the results of VVI.

When a subject is used to wearing glasses, then his VOR will be adapted to the degree of optical correction (Rönne, 1923). Although in principle the IRIS system can be used when the subject is wearing glasses (which automatically compensates for this effect), in practice that places the detector too far from the eye to give good readings. This forced us to measure without glasses, and so eye movements (and thus the VOR gain) were not obtained in a compensating way. The magnification factor is given by (Westheimer, 1986, p 4-9):

$$
\begin{aligned}
& m=\frac{l}{l \cdot(1-h \cdot F)+h^{2} \cdot F} \\
& 1 \text { (letter) distance of target to the eye (in } m \text { ) } \\
& \text { m magnification factor of the lens. }
\end{aligned}
$$

One of the controls used -6.5 diopter, which (with $\mathrm{l}=1.0 \mathrm{~m}$ and assuming $\mathrm{h}=0.027$ m) should decrease his VOR gain by $15 \%$. This was found indeed in a specially calibrated measurement. Although it is easy to correct computationally for these effects, this was not done, because calibration problems (chapter 3.3) did not allow us to use gain values per se on a routine basis, and gain contrast is not influenced by these effects.

More troublesome is the finding of Demer (1981) that for a given low frequency $(0.05 \mathrm{~Hz}$ in his case) the phase shift of the VOR in the dark for cats depends on the gain. If this would also be the case for higher frequencies for man, than these effects should be accounted for.

Using a television screen to present a visual stimulus, makes the fixation distance dependent on the stimulus amplitude (see appendix 14.2 "Viewing a nearby target"):

$$
\begin{aligned}
& d=-f+\sqrt{r^{2}+(r+f)^{2}-2 \cdot(r+f) \cdot \sqrt{r^{2}-a^{2}}} \\
& \text { a stimulus amplitude } \\
& \text { d } \quad \text { difference in fixation distance } \\
& \text { r radius of tv-seceen curvature }
\end{aligned}
$$

Theoretically, this might be important, as Hine \& Thorn (1987) have shown that the angle of convergence (which depends on the fixation distance) modulates the VOR. 
In our situation ( $\mathrm{f}=1 \mathrm{~m}, \mathrm{r}=1 \mathrm{~m}$, and $\mathrm{a}=0.15 \mathrm{~m}$ ), the variation in distance about $2.2 \mathrm{~cm}(2.2 \%)$, making any alteration in VOR gain negligible.

\subsection{Group 3 might consist of 2 sub groups.}

As not yet a golden standard is available for vestibular pathology, the patients were evaluated on basis of their anamneses and their performance at standard tests. The current data, however, hint at a slightly different classification.

Twelve of the 21 Normal probability plots strongly suggest that group 3 (peripheral vestibular dysfunction without complete central compensation) actually consists of two distinct sub groups. Those two sub groups appear to have different variances for the eftVVIT phase shift at $0.41 \mathrm{~Hz}$, for the eft- and mtVVIT coherence at $0.16,0.41$ and $1.15 \mathrm{~Hz}$, for the VVIT gain contrast at $0.41 \mathrm{~Hz}$, for the VVIT phase shift difference at 0.41 and $1.15 \mathrm{~Hz}$, for the VVIT coherence contrast at $0.41 \mathrm{~Hz}$, and to have different means for the mtVVTT phase shift at $1.15 \mathrm{~Hz}$ (table 13-1).

Figure 13-1 shows some examples of the detrended Normal probability plots. Deviations of Normality like these can, of course, occur by chance, although it is unlikely that so many clear distinctions appear for only one group.

It was checked how consistently subjects belonged to one sub group or the other. Hereto, each subject was assigned $-1,1$, or 0 for each variable-frequency combination, depending on whether he belonged to the group having low or high values, or was at the intersection of those two groups (figure 13-1, table 13-2). If subjects produced low or high values by chance, than most subjects' sums should be around zero. But, at the

Tabe: 13-1. Varimblefrequency combinations which suggest in Normal probability plots that two sub groups exist within groulp 3 (untalestal peripherai vestibular dysfunction without complete central compensavion) Var = sub groups differ in wariance; caean - sub droups heve different mearis: - $=$ no clear distinction of sub growps.

\begin{tabular}{|c|c|c|c|}
\hline & $0.16 \mathrm{~Hz}$ & $0.41 \mathrm{kz}$ & 1. $1.5 \mathrm{~Hz}$ \\
\hline efinVIT phase shift & $\ldots$ & var & -. \\
\hline 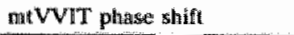 & - & $-\cdots$ & mean \\
\hline efiVVTT coherence & War & var & var \\
\hline muVIT coherence & war & var & var \\
\hline WTT gain contrast & War & 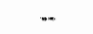 & $\therefore \rightarrow$ \\
\hline VVIT phase shift difference & $\sim$ & vali & var \\
\hline VVIT coherence contrast & - & var & -. \\
\hline
\end{tabular}



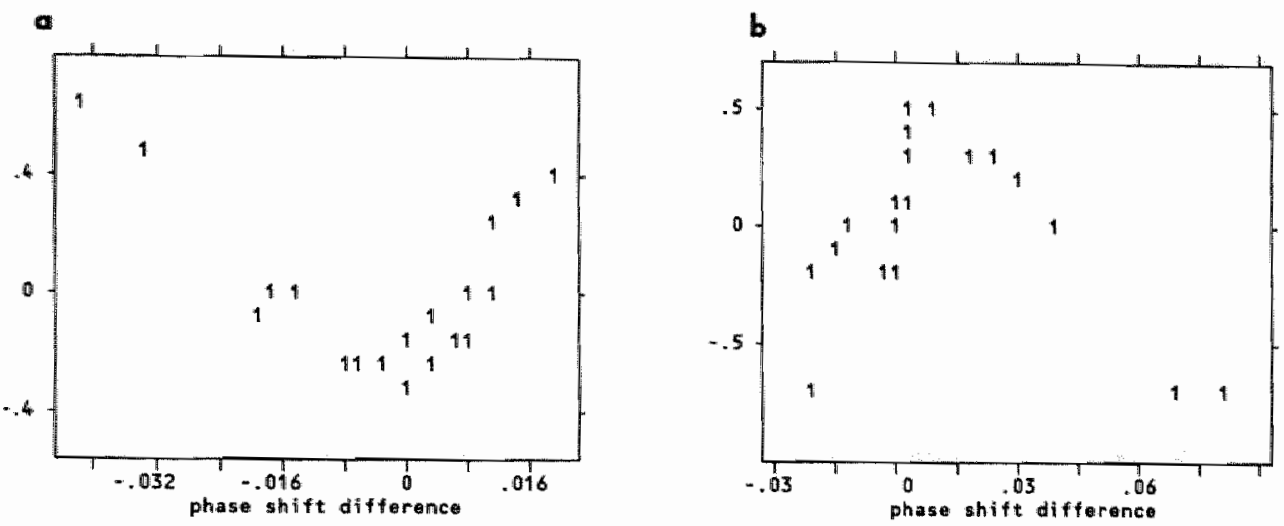

Fig 13-1. Detrended Normall probability plots, showing deviations from expected Normal values for phase shift differences for group 3 at a. stimulus frequency $\mathrm{f}=0.41 \mathrm{~Hz}, \quad$ b. stimulus frequency $f=1.15 \mathrm{~Hz}$.

other hand, the more consistently a subject belongs to a group, the more extreme the score would be. The distribution of the consistency scores can be assessed by an histogram. Three histograms were made: one for the direct variables, one for the relative variables (individual contrasts and individual difference) and one for the combination of those two (figure 13-2).

A distinction of sub groups for the direct variables may disappear when calculating the contrasts, if the sub groups differ for the two conditions in exactly the same way. And therefore the distributions of the consistency scores are assessed separately for the direct and the relative variables. Furthermore, it is possible that diagnostic group 3 consists of four sub groups $a, b, c$, and $d$, of which $a$ and $b$ differ from $c$ and $d$ for the direct variables, and $a$ and $c$ differ from $b$ and $d$ for the relative variables. To keep the analysis simple and also in view of the small sample size $(n=13)$, this is not pursued further.

Because in relative measurements high or low values depend on the arbitrary ordering of terms in the subtraction, subjects consistently producing high values in
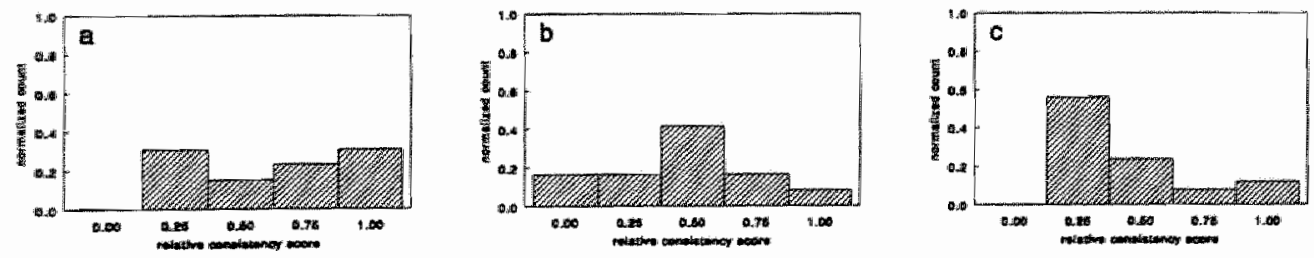

Fisg. 13-2. Normalized histograms of the relative consistency scores for 2 the direct variables, b. the relative variablles, $c$ all variables combined. 


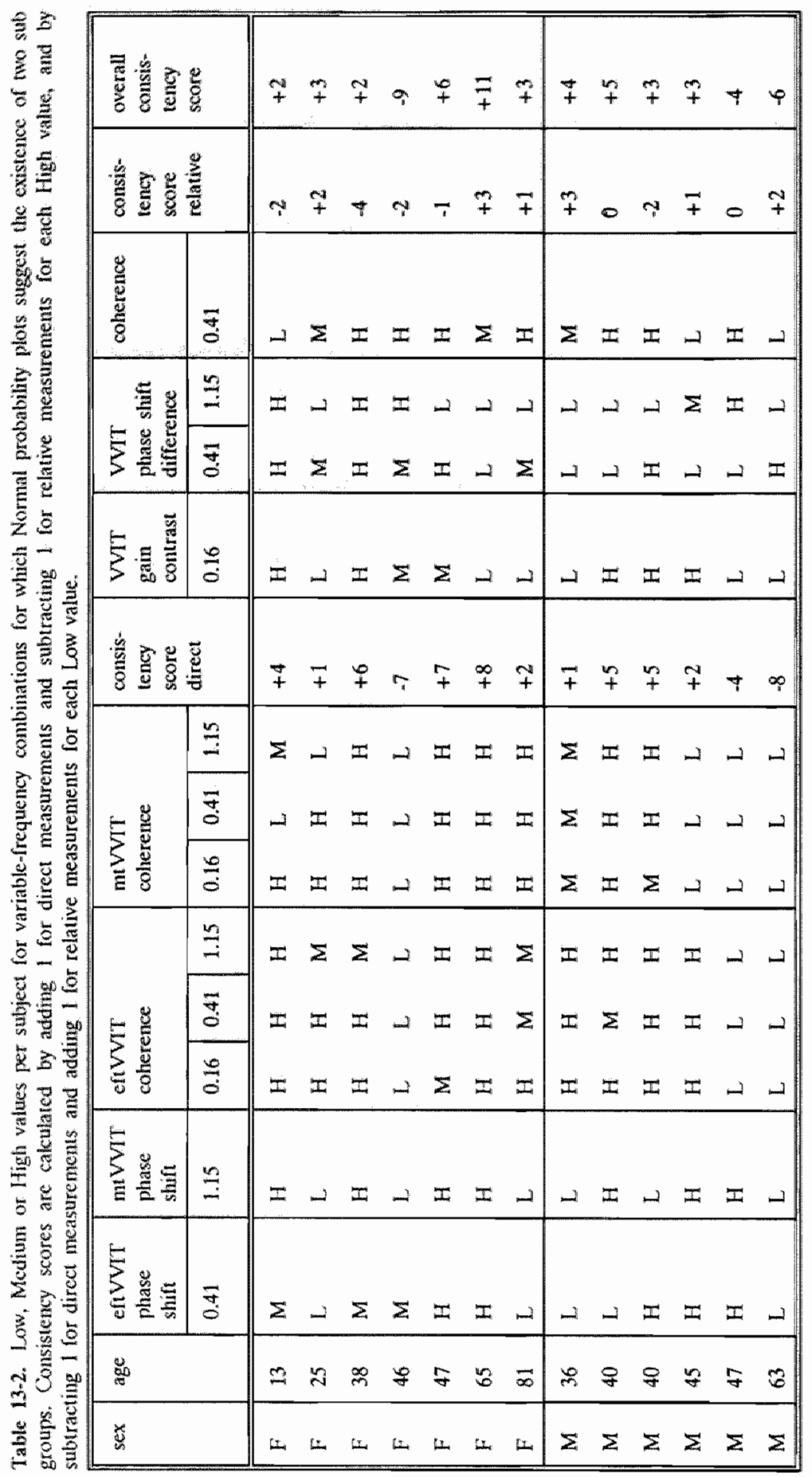


Tabie 13-3. Significances of effect of age on VWTr results, expressed as probabilitic that the obserwed wariations are ajue to

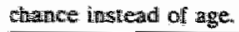

\begin{tabular}{|c|c|c|c|c|c|c|}
\hline $\begin{array}{l}\text { gaitu } \\
\text { contrasit }\end{array}$ & $\begin{array}{l}\text { efif } \\
\text { phase: }\end{array}$ & $\begin{array}{l}\text { Int } \\
\text { phase: }\end{array}$ & $\begin{array}{l}\text { Phase } \\
\text { ditherence }\end{array}$ & $\begin{array}{l}\text { eft } \\
\text { coherence }\end{array}$ & $\begin{array}{l}\text { mi } \\
\text { coherency }\end{array}$ & $\begin{array}{l}\text { woherence } \\
\text { conthraxt }\end{array}$ \\
\hline 0.242 & 0.251 & 0.283 & 0.415 & 0.431 & 0.237 & 0.399 \\
\hline
\end{tabular}

direct measurements, can consistently produce low values in relative measurements. As this appeared indeed to be the case in our situation, we reversed the sign of the consistency scores of the relative measurements (adding 1 for each low value and subtracting 1 for each high value). To facilitate comparison of the three histograms, the $x$-axis was converted to a relative consistency score (score as a fraction of the maximum possible) and the counts are normalized (all classes sum up to unity). Finally, interpretation was made easier by folding the histogram over at zero by taking the absolute values of the relative consistency scores. In this way, the score ranges from 0 (no consistency at all; perfectly random) to 1 (perfectly consistent; no randomness at all). From figure 13-2 it is clear that at least one of the histograms shows a main peak off zero (in fact, all three have their peaks off zero). It is concluded, therefore, that some subjects of group 3 have results consistently different from those of the others, i.e., group 3 consists of two distinct sub groups.

It is remarkable that group 3 is the only one for which the existence of two sub groups is suggested and that it is the only one for which the age distribution is markedly non-Normal (chapter 3.1). Still, age is not found to be even a bit suggestive: the probabilities that chance instead of age caused the observed variation in VVIT results, exceeded 0.23 for all variables (table 13-3).

Forgetting about the age distribution and assuming diagnoses are correct, one could speculate that one sub group has not yet any central compensation, while the other has already started to compensate. If so, then one of the sub groups should be rather similar to group 2 (peripheral vestibular dysfunction, well compensated); this was not investigated further.

\subsection{Interpretation phase lead.}

A remarkable feature of mtVVI responses is the phase lead for the chair-delivered frequencies. Comparing the almost zero phase shift of the responses to sum-of-sine eftVVI stimulation (visually enhanced VOR; chapter 6.4), shows that the mVVVI phase lead does not result from prediction. 
The average phase shifts of the sum-of-sine mtVVI measurements closely match those of single-sine hftVVI (fixation suppression) measurements (Hydén et al. 1982; Larsby et al., 1984; Möller et al., 1990; Kasteel-van Linge \& Maas, 1990). Two inferences can be made from this.

First, mtVYI resembles hftVVI (VOR suppression) more than either eftVVI (visually enhanced VOR) or VOR in the dark.

And second, sum-of-sine randomness of the mtVVI stimulus does not affect respon ses. This latter is in accord with the finding of Barnes \& Eason (1988) that gain and phase of VOR responses in the dark are unaffected by the highest frequency in a sum-of-4sines stimulus (as opposed to smooth pursuit responses).

Two different schemes have been proposed to explain the phase lead in case of VOR suppresion.

Barr et al. (1976) and Baloh et al. (1984) suppose that the lead results from the velocity-coding vestibular signal being used directly to position the eyes, instead of being put through the neural integrator.

At the other hand, Barnes et al. (1978) propose that the lead results from the smooth pursuit system reacting to the retinal slip caused by the (for hftVVI inappropriate) VOR. Using the model given in the introduction (figure 2-4 of chapter 2.2), and lumping the visual feedback gain and the visual delay with the other parameters from the visual feedback loop descriptor $F$, the authors describe the eye movement in the head by

$$
\begin{aligned}
\dot{\theta}=-\frac{G+F}{1+F} & \cdot \dot{\phi}+\frac{F}{1+F} \cdot \dot{\Psi} \\
\dot{\theta} & \text { eye velocity in head } \\
\dot{\varphi} & \text { head velocity } \\
\dot{\Psi} & \text { target velocity } \\
F & \text { lumped parameter representation of visual system } \\
G & \text { lumped parameter representation of vestibular system }
\end{aligned}
$$

In case of hftVVI (VOR suppression) target velocity equals head velocity, leading to

$$
\dot{\theta}_{h f}=-\frac{G}{1+F} \cdot \dot{\phi}
$$




$$
1-\frac{\dot{\theta}_{h r t}}{\dot{\theta}_{d r k}}
$$

and using $F=0$ (no visual feedback) for the VOR in the dark

$$
\dot{\theta}_{d r \dot{k}}=-G \cdot \dot{\psi}
$$

gives

$$
1-\frac{-\frac{G}{1+F} \cdot \dot{\phi}}{-G \operatorname{si}}=\frac{F}{1+F}
$$

for the efficiency of the VOR suppression.

The smooth pursuit response is obtained when the head velocity is zero

$$
\dot{\theta}_{s p}=\frac{F}{1+F} \cdot \dot{\psi}
$$

so that the authors conclude that the efficiency of VOR suppression equals the efficiency of smooth pursuit

$$
1-\frac{\dot{\theta}_{h t}}{\dot{\theta}_{d r k}}=\frac{\dot{\theta}_{s p}}{\dot{\psi}}
$$

Assuming that the relation between the smooth pursuit response and the target movement is given by the complex function $\underline{c}$ (depending on frequency), the complex relation $d$ between VOR suppression and VOR in the dark can be written as

$$
d=1-c
$$




\section{Using}

$$
c=c \cdot e^{i \cdot \phi}=c \cdot(\cos \phi+i \cdot \sin \phi)
$$

the real and inaginary parts of $\underline{d}$ are given by

$$
\begin{aligned}
& \mathfrak{A}\{1-c\}=1-c \cdot \cos \phi \\
& \{\{1-c\}=-c \cdot \sin \phi
\end{aligned}
$$

so that the gain of $\mathrm{d}$ is

$$
|1-c|=\sqrt{\Re\{1-c\}^{2}+\mathfrak{X}\{1-c\}^{2}}=\sqrt{1+c^{2}-2 \cdot \cos \phi}
$$

and the phase is

$$
\Delta(1-c)=\operatorname{arctg} \frac{\Im\{1-c\}}{\Re\{1-c\}}=\operatorname{arctg} \frac{-c \cdot \sin \phi}{1-c \cdot \cos \phi}
$$

If, for example, the smooth pursuit response for a certain frequency has a gain of 0.95 and a phase shift of $-5^{\circ}$, then the amplitude ratio between eye movements caused by hftVVI stimulation (fixation suppression) and VOR in the dark would be

$$
|d|=\sqrt{1+0.99^{2}-2 \cdot 0.99 \cdot \cos \left(-10^{\circ}\right)}=0.17
$$

and the phase shift

$$
\Delta d=\operatorname{arctg} \frac{-0.99 \cdot \sin \left(-10^{\circ}\right)}{1-0.99 \cdot \cos \left(-10^{\circ}\right)}=+7^{\circ}
$$

It was tried to simulate (PSI/e, BOZA automatisering BV, Pijnacker, The Netherlands $\mathrm{mtVVI}$ with the model given in the introduction with values obtained from the literature, but the visual feedback loop (figure 13-3a) oscillated at each second half cycle (figure 13-4b). The oscillation did not depend on the sign of the stimulus, as using $-\sin 2 \pi f t$ instead of $\sin 2 \pi f t$ showed. Neither did it depend on the method of integration or the step size of integration. This has not been investigated further. 


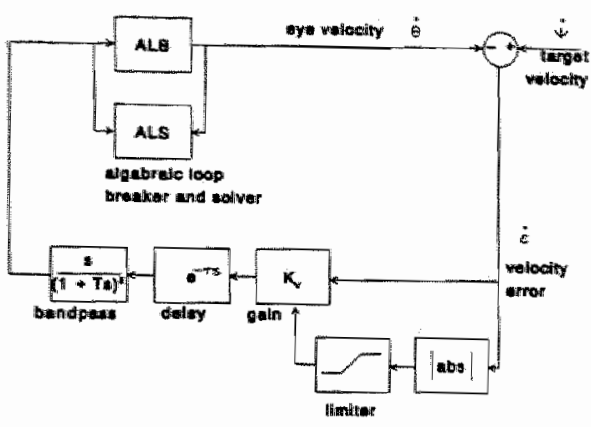

a

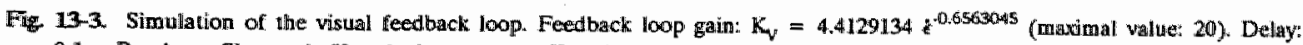
$T=0.1 \mathrm{~s}$. Bandpass filter gain $K=1$, thime constant $T=0.75 \mathrm{~s}, \mathrm{a}$ modiel. b. result.

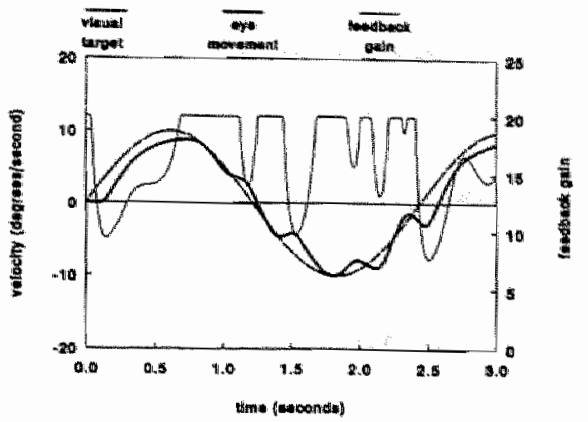

b

\subsection{Separation of vestibular patients and normals by the Visuo-Ves- tibular Interaction Test.}

Irrespective of how VVI works, it was shown to allow separation of vestibular patients and normals, while sex and age were not found to influence VVIT results significantly (chapters $8 \ldots 10$ ). This latter agrees with the VOR gain study by Wall III et al. (1984). They found that normal VOR gain decreases slightly with age for sinusoidal rotation in the dark, but significantly so only at frequencies of $0.01 \mathrm{~Hz}$ and lower. Females were found by them to have higher gains than males at $0.01 \mathrm{~Hz}$ and lower, but this was statistically insignificant. Effects on phase were even less than effects on gain. Our VVIT stimuli were in the $[0.16 . .1 .15] \mathrm{Hz}$ frequency range and so well above the frequencies where they found sex and age related effects. It agrees also with the SP study by Yu et al (1990), who did not found any significant differences caused by sex or age.

Table 13-4 recapitulates the significances of the found differences between the patient groups and the group of normals, from which several observations can be made.

The patients having benign paroxysmal positioning vertigo (group 6) and the whiplash patients (group 4) form the two groups having the highest number of deviant variables. But because the former was a very small group $(n=3)$, their outcome has to be viewed with some caution. The latter scores deviantly on all three types of variables (gain, phase shift and coherence). Although the VVTT was devised for vestibular function testing, it can be used to test integration as well. So it should be 
Table 13-4. Lowsest chancte probabillies (chosen from the interaction of dibgnosis and stimulus frequency, and diagnosis after interactionj townd for the combinations pof patient groups and test parameters. Group sizes ame given th parenthesises probabilitis of so and betlow are printed in bold.

\begin{tabular}{|c|c|c|c|c|c|c|}
\hline & $\begin{array}{l}\text { VWT } \\
\text { gain con } \\
\text { trast }\end{array}$ & $\begin{array}{l}\text { milVIT } \\
\text { phate: }\end{array}$ & $\begin{array}{l}\text { VWTT } \\
\text { phase shif } \\
\text { difference }\end{array}$ & $\begin{array}{l}\text { miVVIT } \\
\text { coherence }\end{array}$ & $\begin{array}{l}\text { VWIII } \\
\text { cohterence } \\
\text { contrast }\end{array}$ & aweraged \\
\hline 1. Mentier (4) & $0,009 \quad(4)$ & $0.138(4)$ & 0.042 & 0.002 (4) & $0.016 \quad 4)$ & 0.059 \\
\hline 2. compensated (8) & $0.021 \quad(8)$ & 0.054 & $0.165 \quad(7)$ & $0.062(7)$ & $0.011 \quad(6)$ & 0.063 \\
\hline 3. uncompensated (13) & 0.038 (10) & 0.723 (12) & $0.401(12)$ & 0.031 (13) & 0.023 (11) & 0.243 \\
\hline 4. Whiplast (10) & $0.053 \quad(9)$ & 0.037 & $0.158 \quad(8)$ & $0.001 \quad(8)$ & $0.006 \quad(9)$ & 0.051 \\
\hline 6. BPPD (3) & $0.064 \quad(3)$ & 0.004 & $0.053 \quad$ (3) & $<0.0005$ (3) & $<0.0005$ & 0.024 \\
\hline 7. unknown causes $(4)$ & $0.118 \quad$ (3) & $0.117 \quad(4)$ & $0.095 \quad$ (4) & $0.067 \quad$ (4) & $0.324 \quad$ (4) & 0.144 \\
\hline
\end{tabular}

no surprise that the whiplash patients, who are known to have trouble integrating multisensorineural information, score differently from normals. Given the difficulty to quantify whiplash trauma, this functional approach might become important in objectifying their complaints (which can be important in settling legal matters concerning damage compensation, as most whiplash trauma is caused by car accidents).

The next group is formed by patients having a well compensated unilateral vestibular dysfunction (group 2). This groups too deviates for all three types of variables. The fact that they have also deviant gain contrast values, is a bit unexpected, because they are labeled "well compensated" for having normal gains in standard tests. Because standard tests evaluate the vestibular system below $0.1 \mathrm{~Hz}$ and the VVIT does so in the $[0.16 .1 .15] \mathrm{Hz}$ range, this might imply that central compensation may be limited to the lower frequencies. If so, then the difference between these patients and controls would be most significant for the interaction of diagnosis and stimulus frequency. Table 8-5 shows the opposite to be the case: the probability that the diagnostic groups have equal means, is after accounting for interactions 0.021. (making the difference significant at the $5 \%$ level), while the probability that the observed differences are due to chance (instead of to the interaction of diagnosis and stimulus frequency) is 0.808 . It still is possible that the frequency dependency of the central compensation ends before the $0.16 \mathrm{~Hz}$ at which the VVIT starts, but another possibility is that an again normal gain in standard tests does not necessarily mean that the VVIT gain should be normal too. After all, the normal gain for torsion in the dark is circa 0.7 , and so complete central compensation at that level still falls short of the normal VVIT gain of 1.1. This matter has not been pursued further.

Patients in the early stages of Meniere's syndrome (group 1) come next in terms of 
deviation, followed by those having incompletely compensated unilateral vestibular dysfunctioning (group 3).

Finally, patients for whom no cause for their balance complaints is known (group 7) differ only suggestively, but not significantly at the $5 \%$ level, from the controls. At the one hand, it can be seen as consistent with standard tests that the VVIT could not objectify the complaints of patients with no known cause, but at the other hand it is disappointing that the VVIT does not add evaluating capabilities in this respect. VVIT phase shift difference and mtVVIT coherence values, however, are suggestively different, and together with the small sample size $(n=4)$, this asks for further study. Of the variables, coherence contrast is most indicative of vestibular abnormality in case of balance complaints, followed by mtVVIT coherence. But because gain contrast, mtVVIT phase shift and phase shift difference are more specific with respect to types of dysfunction, they might have more practical use.

Based on the frequency-averaged sums of squared deviations from unity for sensitivity and specificity, the groups of Meniere patients and whiplash patients are best recognized and differentiated, followed by those having BPPD (table 13-5). Looking at the variables-averaged results, both the MANOVA and the sensitivity and specificity analysis agree that the three most distinctive groups are the whiplash patients, the Meniere patients, and the BPPD patients. The differences between the two types of analyses with respect to the order of those groups ([BPPD, whiplash, Meniere] or [whiplash, Meniere, BPPD], in decreasing degree of deviating from the controls), may stem from the different comparisons (patients versus controls only, or patients versus other patients and controls), from non-Normal distributions, from unequal variances, or may have happened by chance due to the extremely small group sizes.

Table 13-5. Frequency-averaged sums of squared dewiations from unity for sensitivity and specificity per patient group and per variatble. Values below 0.50 are printed in bold.

\begin{tabular}{|c|c|c|c|c|c|c|}
\hline & $\begin{array}{l}\text { WVIT } \\
\text { gatin con- } \\
\text { trast }\end{array}$ & $\begin{array}{l}\text { mivVIT } \\
\text { phase }\end{array}$ & $\begin{array}{l}\text { VVTr } \\
\text { phase shift } \\
\text { difference }\end{array}$ & $\begin{array}{l}\text { mivvT } \\
\text { coherence }\end{array}$ & $\begin{array}{l}\text { VVrt } \\
\text { coherence } \\
\text { contrast }\end{array}$ & averaged \\
\hline 1. Meniere & 0.33 & 0.36 & 0.64 & 0.46 & 0.22 & 0.40 \\
\hline 2. compensated & 0.62 & 0.50 & 0.53 & 0.65 & 0.81 & 0.62 \\
\hline 3. uncompensated & 0.63 & 0.78 & 0.75 & 0.47 & 0.58 & 0.64 \\
\hline 4. whiplash & 0.33 & 0.50 & 0.42 & 0.45 & 0.29 & 0.40 \\
\hline 6. $\mathbb{B P P D}$ & 0.63 & 0.50 & 1.06 & 0.45 & 0.36 & 0.60 \\
\hline 7. unknown causes & 0.61 & 0.80 & 0.73 & 0.75 & 0.57 & 0.69 \\
\hline
\end{tabular}




\subsection{Conclusions.}

We set out to develop a vestibular function test whose results would generally agree with the outcomes of the standard tests, but which would have less variability and which would correlate better with the complaints of the patients. The results presented here, seem to indicate that we have succeeded in achieving our first two goals.

Apart from the side localization in case of peripheral vestibular dysfunctions, the VVIT results correspond to those of the standard tests (chapter 11).

In order to asses whether VVIT results show less variation than the standard tesit results, the intra- and inter-individual variabilities for patient groups and for normals should be compared for both tests. That has not yet been done; only normal VVTT results have been thus analyzed (chapters 5 .. 7). Nevertheless, this report allows some inferences to be made. The fact that the subjects can be better classified using VVIT results than using standard test results (chapter 11), suggests that the former may have less variable results. Also the sensitivities point in that direction (table 13-6). Except for the best and the average scores for the centrally compensated peripheral vestibular patients and all scores for the patients with unknown causes, all combinations of diagnostic groups and sensitivity measures are better for the VVIT than for the standard tests (gain of torsion in the dark, asymmetry of torsion in the dark, left caloric response, right caloric response, asymmetry in caloric responses). (Using the standard reference range for the VVIT; when using the unbiased reference range, VVIT performance is even better). The specificity of the standard test for our control population is not known, but this is not important, as the VVIT specificity is 1.00 .

Correlations between complaints and test outcomes, however, may be equally poor

Table 136. Sersitivities of the standard lests and of the VWTT, using standard reference ranges. The sensitivity has bert computed in three ways: the best sifigle result per subject was used ("best") no distinction was made berween test resulth per

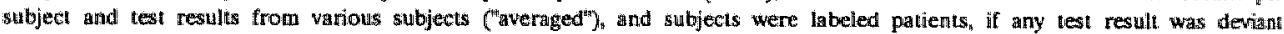

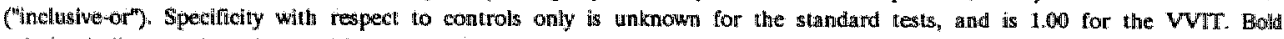
printing indicates wilues that are thigher than those of the other test.

\begin{tabular}{|c|c|c|c|c|c|c|}
\hline & \multicolumn{3}{|c|}{ sitandlard tests } & \multicolumn{3}{|c|}{ WVIT } \\
\hline & best & aweraged & incl. or & best & averaged & incl or \\
\hline 1. Mentere & 0.50 & 0.15 & 0.50 & 100 & 051 & 11.00 \\
\hline 2. compensated peripheral & 0.86 & 0.46 & 0.86 & 0.75 & 0.34 & 0.88 \\
\hline 3. uncompensated periplieral & 0.54 & 0.22 & 0.69 & 0.92 & 0.35 & 1.00 \\
\hline 4. whiphash & 0.44 & 0.16 & 0.44 & 100 & 0.51 & 1.00 \\
\hline 6. $\mathrm{BPPD}$ & 0.00 & 0.00 & 0.00 & LeO & 0.42 & 1,00 \\
\hline 7. unknown causes & 0.67 & 0.35 & 0.67 & 0.50 & 0.30 & 0.50 \\
\hline
\end{tabular}


for the VVIT and the standard tests. Due to the limited time available, this impression has not yet been substantiated by analyses. 
160 


\section{Appendices.}

\subsection{Sum-of-sines pseudo-random stimuli.}

Summing sines of different frequencies makes the signal appear random for short lengths of time, but ultimately the signal will repeat itself. If the period of a sum-ofsines signal equals the length of a data segment, and if the studied system is nonlinear (as all practical systems are, especially biological ones), then the estimates of the transfer function and of the coherence function will be biased (Maki, 1986). Due to the system's nonlinearities, the bias in the transfer function occurs at frequencies that are multiples of the input frequencies. So by evaluating the frequency spectrum only at frequencies at which is stimulated and by having input frequencies that are no multiples of each other, unbiased estimates of gain and phase shift may be obtained. However, the system's nonlinearities not only produce harmonic distortion, but also generate intermodulation distortion (Maki, 1988). So as an additional requirement, none of the input frequencies should be a multiple of the other's sums and differences. The three frequencies of the chair movement $(0.16,0.41$, and $1.15 \mathrm{~Hz})$ and the one of the visual target movement $(0.53 \mathrm{~Hz})$ together satisfy these constraints almost,

Table 14-1. Stimulus frequencies are harmonically unrelatted to each other and to their pairwise sums (except one: the lourth frequency is almosi twice the sum of the first two frequencies) and difierences. Tabulated are the ratio's (highest value)/(lowest value).

\begin{tabular}{|c|c|c|c|c|c|c|}
\hline & & & \multicolumn{4}{|c|}{ stimulus frequency } \\
\hline & & & freq 1 & freq 2 & freq 3 & freq 4 \\
\hline & & & 0.16 & 0.4 .1 & 0.53 & 1.15 \\
\hline \multirow[t]{4}{*}{ stimulus frequency } & freq 1 & 0.16 & & & & \\
\hline & freq 2 & 0.41 & 2.6 & . & & \\
\hline & freg 3 & 0.53 & 3.3 & 1.3 & & : \\
\hline & freq 4 & 1.15 & 7.2 & 28 & 22 & \\
\hline \multirow[t]{6}{*}{ difference } & $|\mathbf{2}-\mathbb{1} 1|$ & 0.25 & 1.6 & 1.6 & 2 & 4.6 \\
\hline & $|\mathbf{3}-||1|$ & 0,37 & 23 & 1.1 & 1.4 & 3.1 \\
\hline & $|14 \cdot \| 1|$ & 0.58 & 6.2 & 24 & 1.9 & 1.2 \\
\hline & $|\mathbf{S}-| 2 \mid$ & 0.12 & 13 & 3.4 & 4.4 & 96 \\
\hline & $|14=12|$ & 0.74 & 4.6 & 1.8 & 1.4 & 1.6 \\
\hline & $\| \mathbb{C}-13 \mid$ & 0.62 & 3.9 & 1.5 & 12 & 1.9 \\
\hline \multirow[t]{6}{*}{ sumen } & $\mathrm{R}+\mathrm{IH}$ & 0.57 & 3.6 & 1,4 & $\mathbb{1 1}$ & 20 \\
\hline & $\mathrm{f}+\mathrm{I}$ & 0.69 & 4.3 & 11.7 & 1.3 & 1.7 \\
\hline & $(4)+\mathbf{n}$ & 1.31 & 8.2 & 3.2 & 2.5 & 1.1 \\
\hline & $B+2$ & 0.94 & 5.9 & 2.3 & 1.8 & 1.2 \\
\hline & $14+2$ & 1.56 & 9.8 & 3.8 & 29 & 1.4 \\
\hline & $f 4+8$ & 1.68 & 10.5 & 4.1 & 32 & 1.5 \\
\hline
\end{tabular}


as shown in table 14-1. Only one combination is harmonically related (the fourth frequency is almost twice the sum of the first two frequencies).

\subsection{Viewing a nearby target.}

Viewing a target which is not at infinity, alters the ideal gain from unity to ome slightly greater. Further, the viewing distance varies when the head rotates while the target is earth-fixed, and/or the target moves along an other trajectory than the circle centered at the eye. With the viewing distance, the vergence angle varies, and this might influence the VOR (Hine \& Thorn, 1987).

\section{Gain}

For the following argument a rotating head in combination with an earth-fixed target is equivalent to an earth-fixed head in combination with a target moving along a circular path. Because the latter is easier to analyse, that situation is depicted in figure 14-1. A target movement to the right, viewed by the left eye, is given here, which is equivalent to an ipsilateral head movement while viewing an earth-fixed target. Note that the distances are here not drawn to scale (in order to distribute the lines and symbols more evenly over the available space). Perfect compensation would require the eye to move over an angle $\gamma$ when the target has moved over an angle $\phi:$

$$
\left.\begin{array}{l}
\gamma=\alpha-\beta \\
\alpha=\operatorname{arctg} \frac{d_{v}}{d_{l}} \\
\beta=\operatorname{arctg} \frac{l_{1}}{l_{2}} \\
l_{1}=d_{i} \cdot \cos \phi-d_{r} \\
l_{2}=d_{i} \cdot \sin \phi+d_{l}
\end{array}\right\}-\gamma=\operatorname{arctg} \frac{d_{v}}{d_{l}}-\operatorname{arctg} \frac{d_{t} \cdot \cos \phi-d_{r}}{d_{t} \cdot \sin \phi+d_{l}}
$$

(see figure 14-1 for the legends).

For an ipsilateral target movement (contralateral head movement), one simply considers the right eye by substituting $-d_{1}$ for $d_{1}$ :

$$
\gamma=\pi-\operatorname{arctg} \frac{d_{v}}{d_{l}}-\operatorname{arctg} \frac{d_{t} \cdot \cos \phi-d_{r}}{d_{t} \cdot \sin \phi-d_{l}}
$$




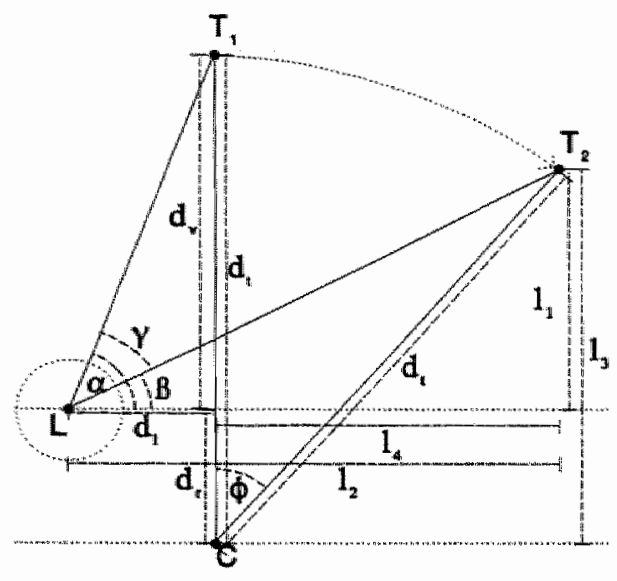

Fig 14 Contralateral tanget movement (equivaleni to ipsilfateral head movemient). When the target moves from $T_{1}$ to $T_{2}$ ower an with $C$ as the center of rotation, then the left eye L should rotate over an angle \% $\mathrm{d}_{\mathrm{w}}=$ viewing distance (plane of eyes' centers to target), $d_{t}=$ target distance (center of headtarget rotation to target), $d_{1}$ laterall distance (eye ${ }^{2} s$ center: to sagital plane), $d_{p}$ rostral distance (plane of centers to center of head/angel rotation), $b_{1}$ to $l_{4}=$ accessory lines denoting various distances..

Some results are given in table 14-2. As a rule of thumb, the gain increase is the reciprocal of $10 \%$ of the viewing distance in $\mathrm{cm}$.

Timble 14-2. Calculated results of ideal compensatory eye movements for several viewing distances and head rotations.

\begin{tabular}{|c|c|c|c|c|c|}
\hline \multicolumn{2}{|c|}{$\begin{array}{l}\text { plane of eyes' centers to center of } \\
\text { rotation }=10 \mathrm{~cm} \\
\text { inter pupillary distance }=6.6 \mathrm{~cm}\end{array}$} & \multicolumn{2}{|c|}{$\begin{array}{l}\text { magnitude of ideal compensalory } \\
\text { eye rotations (deg) }\end{array}$} & \multirow[t]{2}{*}{ mean ideal gain } & \multirow[t]{2}{*}{$\begin{array}{l}\text { right/heft } \\
\text { asymmetry }\end{array}$} \\
\hline $\begin{array}{l}\text { Wiewing distance } \\
\text { (crill) }\end{array}$ & $\begin{array}{l}\text { head ratation } \\
\text { (deg) }\end{array}$ & left eye & right eye & & \\
\hline \multirow[t]{3}{*}{100} & 5 & 5.50 & 5.48 & 1.10 & 0.996 \\
\hline & 10 & 11.02 & 10.94 & 1.10 & 0.993 \\
\hline & 20 & 2207 & 21.78 & 1.10 & 0.9877 \\
\hline \multirow[t]{3}{*}{150} & 5 & 5.34 & 5.32 & 1,07 & 0.996 \\
\hline & 10 & 10.68 & 10.63 & 1.07 & 0.995 \\
\hline & 20 & 21.38 & 21.20 & 1.06 & 0.992 \\
\hline \multirow[t]{3}{*}{200} & 5 & 5.25 & 5.24 & 1.05 & 0.998 \\
\hline & 10 & 10.51 & 10.48 & 1.05 & 0.997 \\
\hline & 20 & 21.04 & 20.91 & 1.05 & 0.994 \\
\hline 300 & 10 & 10.34 & 10.32 & 1.03 & 0.998 \\
\hline 400 & 10 & 10.26 & 10.24 & 1.02 & 0.998 \\
\hline 500 & 10 & 10.20 & 1019 & 1.02 & 0.999 \\
\hline
\end{tabular}




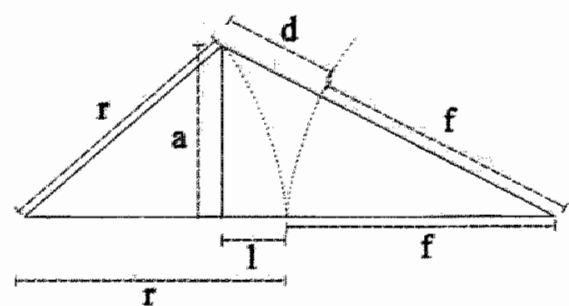

Fig 14-2 Increase of viexing distance when looking at extreme position of visual target. $d=$ increase, $t=$ fration distance, $r=$ screen tadins, $a=$ amplitude of target movement, $1=$ accessory line.

\section{Vergence angle}

The tv-screen used for presenting the visual stimulus has a curvature opposite to the one needed for maintaining a constant fixation distance (figure 14-2, note that the amplitude of the visual stimulus has been grossly exaggerated). Seen from the chair"s center, the increase $d$ in fixation distance is maximal when the target is extreme left or right, and is given by:

$$
\begin{aligned}
& \left.\begin{array}{c}
(f+d)^{2}=a^{2}+\left(f+b^{2}\right. \\
l=r-\sqrt{r^{2}-a^{2}}
\end{array}\right\}- \\
& d=-f+\sqrt{r^{2}+(r+f)^{2}-2 \cdot(r+f) \cdot \sqrt{r^{2}-a^{2}}}
\end{aligned}
$$

(see figure 14-2 for the legends).

Assuming a screen radius and a viewing distance of $100 \mathrm{~cm}$ each, and an amplitude of target movement of $15 \mathrm{~cm}$, results in an increase of fixation distance of $2.2 \mathrm{~cm}$, L.e., an increase of $2.2 \%$

\subsection{Digital differentiation.}

Positional data was digitally differentiated without introducing a phase shift, by:

$$
\begin{aligned}
& x_{t}^{\prime}=\frac{x_{t+n}-x_{t-n}}{2 n \cdot T_{p}} \\
& x_{t} \quad \text { value, sampled at time } t \\
& \text { n number of left or right neighbors in window; } \\
& \text { window width }=2 \mathrm{n}+1 \\
& T_{p} \text { sample period; } T_{p}=t_{i}-t_{i-1} \\
& t \text { point in time }
\end{aligned}
$$


This is equivalent to differencing neighboring samples:

$$
x_{t}^{*}=\frac{x_{t+1}-x_{t}}{T_{p}}
$$

followed by smoothing by taking the running average with unit weights:

$$
\begin{aligned}
x_{t}^{\prime} & =\frac{x_{t+n-1}^{\prime \prime}+x_{t+n-2}^{\prime n}+\cdots+x_{t}^{\prime \prime}+\cdots+x_{t-n+1}^{\prime \prime}+x_{t-n}^{\prime \prime}}{2 n} \\
& =\frac{x_{t+n}-x_{t-n}}{2 n \cdot T_{p}}
\end{aligned}
$$

Approximating differentiation by a digital difference filter changes the amplitudes in a frequency-dependent way, because the filter's transfer function is the sinc function (Hamming, 1983, p 47). Quite probably, median filtering is even more frequency dependent, but, being a non-linear filter, no transfer function is available. So, rather than correct for the lesser effect only, no correction at all is made. This does not give problems, because no frequency-specific gains are compared.

\subsection{MANOVA.}

Multivariate analysis of variance (MANOVA) tests whether observed differences in the mean results of independently defined groups are completely due to chance. Because MANOVA is done by using properties of Normal (Gaussian) distributions, the following requirements have to be met:

1. All groups should consist of random samples.

2. The parent distributions of the combinations of the dependent variables should be multivariate Normal for all groups. (And so, by necessity, the parent distributions of the single dependent variables also have to be Normal for all groups. The reverse, however, is not necessarily true: two Normal distributions may form a nonNormal bivariate distribution).

3. The multivariate parent distributions should have equal variances and equal covariances for all groups (because those parameters are pooled).

Because univariate analyses of variance (ANOVA's) have more statistical power, an additional de facto requirement is that the dependent variables should be (partly) correlated (or, put the other way around: if conditions for an ANOVA are met, but 
the dependent variables are correlated, then a MANOVA is required).

A Normal distribution is a mathematical abstraction, to which very few actual populations conform (few populations have values ranging from minus to plus infinity!). And so the MANOVA requirements are not strict, but allow some deviations, depending on which statistic is being used. The one known as "Pillai's trace" (Norušis, 1986, p B-126):

$$
\tau=\sum_{i=1}^{s} \frac{1}{1+\lambda_{i}} \begin{aligned}
& \text { index variable } \\
& \lambda_{1} \quad \begin{array}{l}
i^{\text {th }} \text { eigenvalue of the product of the hypothesis sums-of-squares and } \\
\text { cross-products matrix with the inverse of the error sums-of-squares }
\end{array} \\
& \begin{array}{l}
\text { and cross - products matrix } \\
\tau
\end{array}
\end{aligned}
$$

is the most robust statistic in the SPSS software, and only this one is used in this study.

For the first condition (random samples), no tests are available to check fulfillment.

The second condition that the distributions have to be multivariate Normal, can beforehand be tested only partly, namely whether the constituting univariate distributions are Normal. If so, their Normal probability plots should show a straight line, and their detrended Normal probability plots should show random variations around zero. Figures 14-3 $a$ and $b$ are examples of a Normal distribution, while figures 14-4 $a$ and
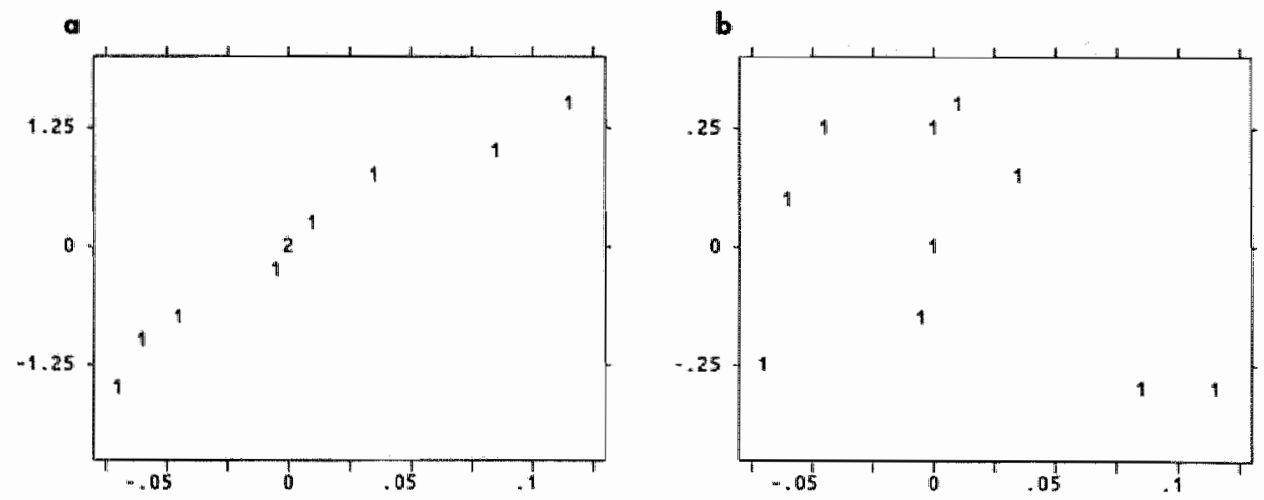

Fig. 14-3 Evaluation of univariate Normality of coherence contrast responses of the whiplash patients (group 4) at the chail. delivered frequency of $0.41 \mathrm{~Hz}$ a. Normal probability plot. b. delrended Normal probability plot. Both indicate a quite: Normal dititibution. 

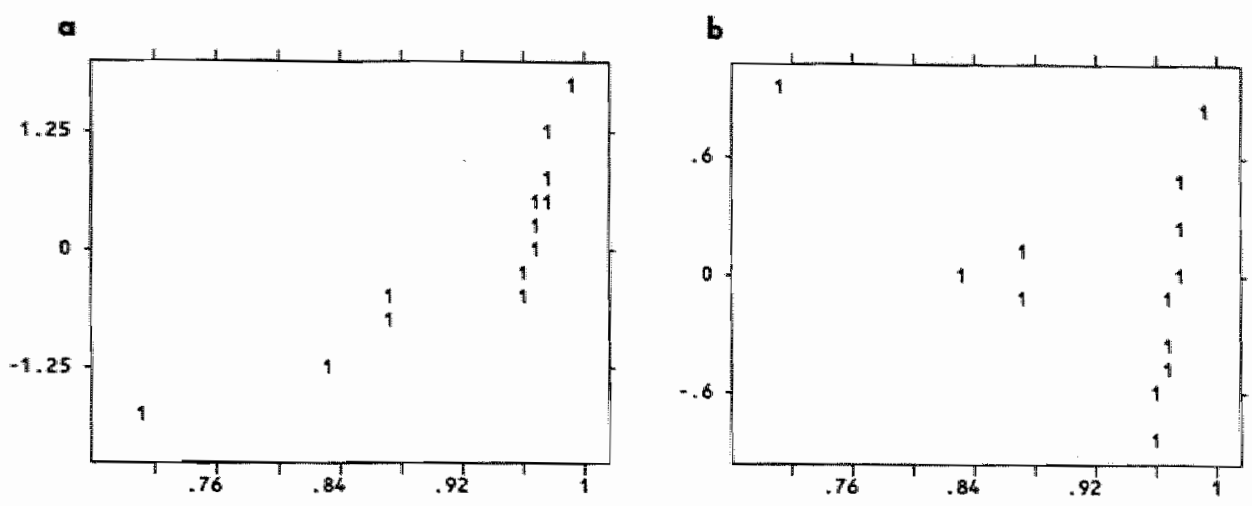

FTy 14-4. Evaluation of univariate Nomality of mtWWT coherence responses of patients with nol comptetely compensated peripheral vestibular dysfunctions (group 3) at the chair-delivered requency of $0.16 \mathrm{~Hz}$ a. Normal probability pliot. b. detrendied Normal probability pliot. Both indicate a serious deviation from Normality.

$b$ show a non-Normal one. After the analysis, the condition can be checked by evaluating the distributions of the residuals (differences between observed and expected values); figure $10-7 \mathrm{f}$ is an example of a Normal residuals' distribution, indicating the bivariate distribution has been Normal, while figure 10-8f shows a markedly non-Normal distribution due to an outlier. For data having no more than three independent variables, the distributions can easily be checked visually (figures $6-4,7-4$, and 10-9).

The third condition of equal variances and equal covariances is tested by assessing the homogeneity of the dispersion (= variance-covariance) matrices. This can be done in a multivariate way by Box's $M$ test, which is based on the determinants of the dispersion matrices in each cell (= group with fixed independent variables) and on the determinant of the pooled dispersion matrix (Norušis, 1986, p B-116). Unfortunately, Box's $M$ test responds to even mild non-Normalities, and furthermore, it can

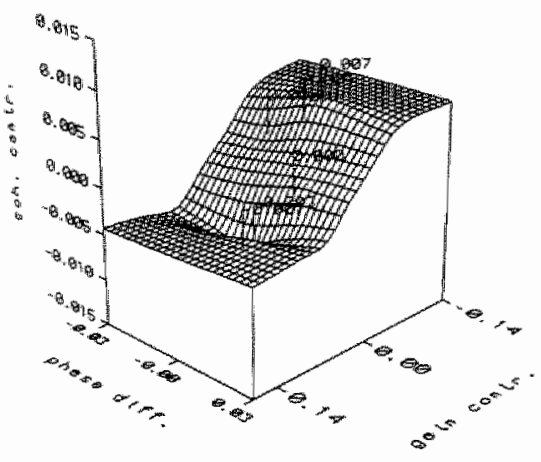

Fig 14-5. Multiwariate plot of frequency-averaged values of 6 normall subjects The univariatie libitits enclose the $95 \%$ standard reference ranges. The values are averaged over frequency, becaluse it is not possible to depict in a convential diagram this correlation as well as that of the three variabitu gain contrast, phase shif difference, and coherence contrast (see however, Geiser et. al. 1993, for multivariate visualization), (gain contrast (Lablie 5-1) and phase shift difference (table 6-1) are independent from frequency). Gain contrast oortelates with phase shif! difference, white coherence contrash correlates with gain contrast, but not with phase shift difference. 

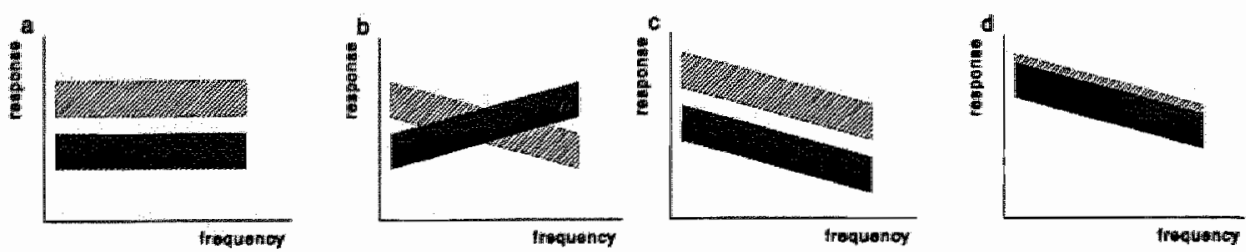

Fig 146. Vartous cluster forms of hypothetical frequency responss of two diagnostically defined groups (different hatchimg) illistrate the interpretation of the MANOWA results from table 1. a. only diagnosis affects the responises significanity: b. thie effect of diagnosis and frecuency on the responses consist solely af their interaction. c both diagnosis and frequency affect the responses significantly, but they do not interact. d only freguency affects the responses significantly.

not be computed when some cells are empty, thus making it a test of limited value for this study. Plotting cell variances and cell standard deviations against cell means allow visual assessment of violations of the requirement for equal variances and equal covariances.

Finally, whether the dependent variables are correlated, can be seen from the correlation matrix. Bartlett's test of sphericity tests the null hypothesis that the variables are completely independent, by assessing how close to zero the determinant of the within-cells correlation matrix is (Norušis, 1986, p B-110). According to this criterium, almost none of analyses in this study was allowed to be done univariate. Figures 5-2 and 6-4 give fine examples of fully correlated responses (for the three chair-delivered frequencies), and figure 14-5 shows how of the three variables gain contrast, phase shift difference and coherence contrast, two correlate with each other, while a third correlates with only one of them but not with the other.

A multivariate analysis tests from the effect of the highest interaction down to the single effects. Human interpretation is often the other way around: single effects are studied, and an interaction is understood as an extra effect on top of the summation of single effects. Figure 14-6 schematically illustrates how hypothetical MANOVA results (table 14-3) of the frequency-dependent responses of two diagnostically defined groups can be interpreted. In general, parallel lines in such graphs indicate

Tible 14-3. Hypotheticil MANOVA results of responses (dependent variable) by the indlependent variables "diagnosis" (having no possible values) and "frequency (contimuous), as depicted in figure 3.

\begin{tabular}{|c||c|c|c|}
\hline figure part & $\begin{array}{l}\text { diagnosis } \\
\text { (after interaction) }\end{array}$ & $\begin{array}{l}\text { interaction of } \\
\text { diagnosis and frequency }\end{array}$ & $\begin{array}{l}\text { frequency } \\
\text { (after interaction) }\end{array}$ \\
\hline significant & significant \\
\hline$b$ & significant & significant \\
\hline$c$ & & significant \\
\hline$d$ & & & \\
\hline
\end{tabular}


the absence of interaction of the plotted variables, and non-parallel lines indicate interaction. 


\section{Abbreviations}

$\gamma$
$\delta$
$\epsilon$
$\lambda$
$\varphi$
$\varphi_{\mu D}$
$\omega$
AD
ADC
ARS
AS
ASCII
BPPD
c
$\mathrm{C}_{\alpha}, C_{\mathrm{j} \cdot a}$

CCW

cftVVI

CW

d

DA

DAC

$\mathrm{DC}$

deg/s

deg

dVOR

eftVAT

eftVOR

eftVVI

ENG

EOG

f

$\mathrm{f}_{e}$

FFT

$\mathrm{Hz}$

coherence

difference in value

error value

parameter ( $\underline{I}$ unbiasing factor, 2 Box-Cox Normalizing correction)

phase shift

phase shift, due to analog-to-digital converter

circular frequency $(=2 \pi f)$

1 right ear, $\underline{2}$ analog-to-digital

analog-to-digital converter

angular rate sensor

left ear

American Standard Code for Information Interchange

benign paroxysmal positioning dizziness

complex number

end points of reference range having a probability $\alpha$ to include a new, independent measurement value

counter-clockwise

visuo-vestibular interaction, with a chair-fixed visual target (= fixation suppression)

clockwise

complex number

digital-to-analog

digital-to-analog converter

direct current

degrees per second

degree

vestibulo-ocular reflex in the dark

vestibular auto-rotation test, with an earth-fixed visual target

vestibulo-ocular reflex, with an earth-fixed visual target (= visually enhanced VOR = eftVVI)

visuo-vestibular interaction, with an earth-fixed visual target (= eftVOR = visually enhanced $\mathrm{VOR}$ )

electronystagmography ( $=\mathrm{EOG}$ )

electro-oculography (=ENG)

frequency

cut-off frequency of filter

fast Fourier transform

Hertz (cycles per second) 


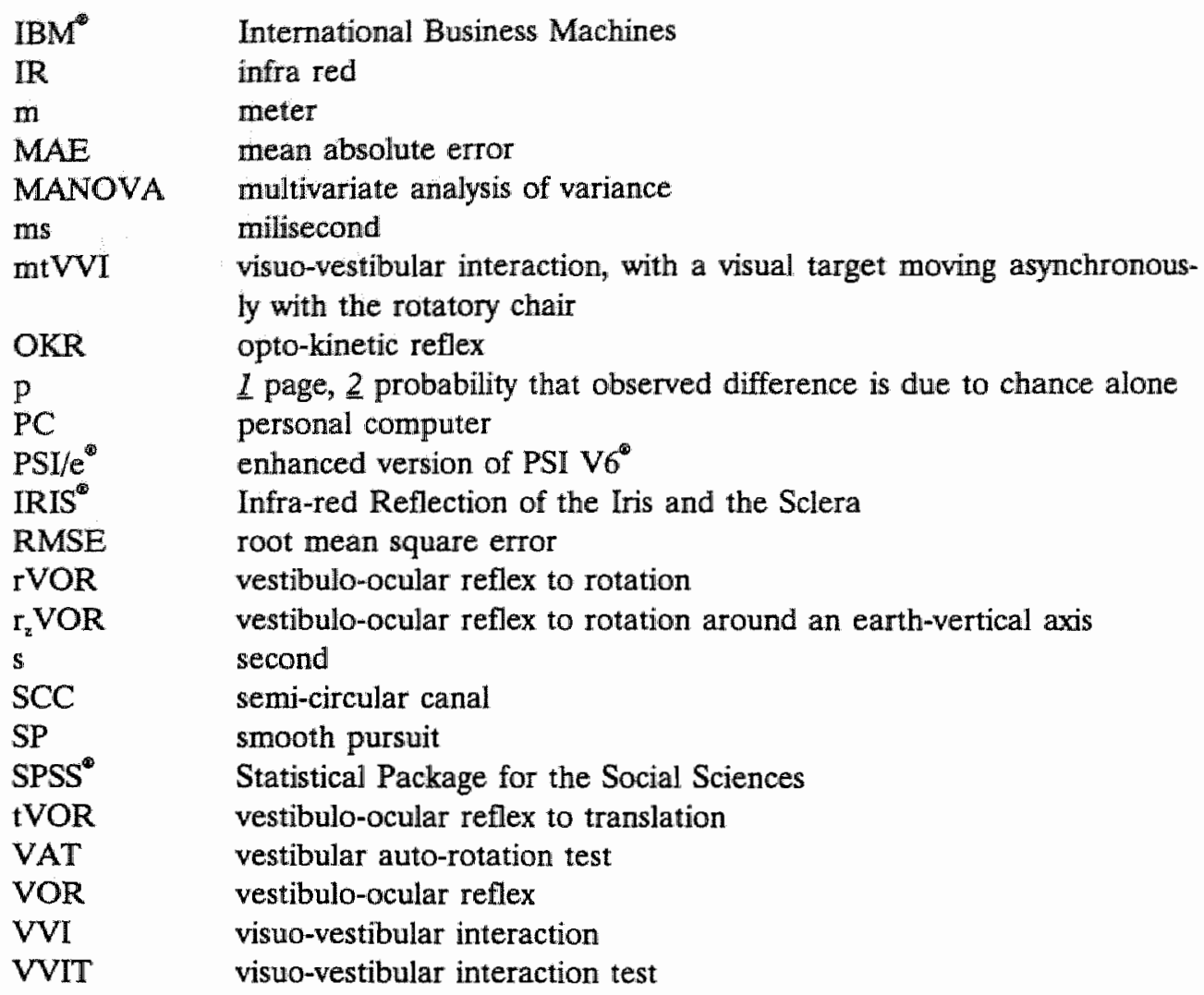




\section{References}

Albert A, Harris EK (1987) Multivariate interpretation of clinical laboratory data. Marcel Dekker, New York, 51-56.

Bahill AT, Iandolo MJ, Troost BT (1980) Smooth pursuit eye movements in response to unpredictable target wave forms. Vision Research 20 923-931.

Baloh RW, Hess K, Honrubia V, Yee, RD (1984) Low and high frequency sinusoidal rotational testing in patients with peripheral vestibular lesions. Acta Otolaryngol (Stockh) Suppl 406 189-193.

Barnes GR, Benson AJ, Prior ARJ (1978) Visual vestibular interaction in the control of eye movement. Aviat Space Environ Med 49 557-564.

Barnes GR, Crombie JW, Edge A (1985) The effects of ethanol on visualvestibular interaction during active and passive head movements. Aviat Space Environ Med 56 695-701.

Barnes GR, Donnelly SF, Eason RD (1987) Predictive velocity estimation in the pursuit reflex response to pseudo-random and step displacement stimuli in man. Joumal Physiol (London) 389 111-136.

Barnes GR, Eason RD (1988) Effects of visual and non-visual mechanisms on the vestibulo-ocular reflex during pseudo-random head movements in man. Joumal Physiol (London) 395 338-400.

Barnes GR, Ruddock CS (1989) Factors effecting the predictability of pseudorandom motion stimuli in the pursuit reflex of man. Joumal Physiol (London) 408 $137-165$.

Barnes GR, Asselman PT (1991) The mechanism of prediction in human smooth pursuit eye movements. Journal Physiol (London) 439 439-461.

Barnes GR (1993) Visual-vestibular interaction in the control of head and eye movement: the role of visual feedback and predictive mechanisms. Progress Neurobiol 41 435-472.

Barr CC, Schultheis LW, Robinson DA (1976) Voluntary, non-visual control of the human vestibulo-ocular reflex. Acta Otolanryngal (Stockh) 81 365-375. 
Bendat JS, Piersol AG (1971) Random data: analysis and measurement procedures. Willey-Interscience, New York.

Benson AJ (1970) Interactions between semicircular canals and gravireceptors. In Recent advances in aerospace medicine (ed Busby DE) D Reidel, Dordrecht, 249

Benson AJ, Barnes GR (1978) Vision during angular oscillation: the dynamic interaction of visual and vestibular mechanisms. Aviat Space Environ Med 49340 345.

Bloomberg J, Melvill Jones G, Segal B (1991a) Adaptive plasticity in the gaze stabilizing synergy of slow and saccadic eye movements. Exp Brain Res 84 (1) 3546.

Bloomberg J, Melvill Jones G, Segal B (1991b) Adaptive modification of vestibularly perceived rotation. Exp Brain Res 84 (1) 47-56.

Bock $O$ (1982) Non-linear interaction of the vestibular and the eye tracking system in man. Exp Brain Res 47 461-464.

Brown BW, Hollander M (1977) Statistics (a biomedical introduction). John Wiley \& Sons, New York, ISBN 0-471-11240-2.

Carpenter RHS (1988) Movement of the eyes. Pion, London, ISBN 0-85086-109-8.

Claremont CA (1931) The psychology of sea sickness. Psyche 11 86-90.

Collewijn H, Martin AJ, Steinman RM (1983) Compensatory eye movements during active and passive head movements: fast adaptation to changes in head magnification. J Physiol (Londen) 340 259-286.

Cullen KE, Belton T, McCrea RA (1991) A non-visual mechanism for voluntary cancellation of the vestibulo-ocular reflex. Exp Brain Res 83 237-252.

Dallas PJ, Jones RW (1963) Learning behaviour of the eye fixation control system. IEEE Trans Auto Control AC-8 218-227.

Demer JL (1981) The variable gain element of the vestibulo-ocular reflex is common to the optokinetic system of the cat. Brain Research 229 1-13. 
Engelken EJ, Stevens KW (1990) A new approach to the analysis of nystagmus: an application for order statistic filters. Aviat Space Environ Med 61 859-864.

Fernández C, Goldberg JM (1971) Physiology of peripheral neurons innervating semicircular canals of the squirrel monkey. II. Response to sinusoidal stimulation and dynamics of peripheral vestibular system. J Neurophysiol 34 661-675.

Furman JM, O'Leary DPO, Wolfe JW (1982) Dynamic range of the frequency response of the horizontal vestibulo-ocular reflex of the alert rhesus monkey. Acta Otolaryngol (Stockh) 93 81-91.

Furst EJ, Goldberg J, Jenkins HA (1987) Voluntary modification of the rotatory induced vestibulo-ocular reflex by fixating imaginary targets. Acta Otolanryngol (Stockh) 103 232-240.

Galiana HL (1985) Commissural vestibular nuclear coupling: a powerful putative site for producing adaptive change in: Reviews of oculomotor research (volume 1) Adaptive mechanisms in gaze control (editors: Berthoz A, Melvill Jones G) Elsevier, Amsterdam, ISBN 0-444-80483-8 327-339.

Geiser FO, Golt C, Kung L, Justice JD, Brown BL (1993) Chromatographic Method Verification by Means of 3-Dimensional, Multivariate Visualization. $J$ Chromatography 631 (1-2) 1-13.

Goebel JA, Fortin M, Paige GD (1991) Headshake versus whole-body rotation testing of the vestibulo-ocular reflex. Laryngoscope 101 695-698.

Goldberg JM, Fernández C (1971a) Physiology of peripheral neurons innervating semicircular canals of the squirrel monkey. I. Resting discharge and response to constant angular accelerations. $J$ Neurophysiol 34 635-660.

Goldberg JM, Fernández C (1971b) Physiology of peripheral neurons innervating semicircular canals of the squirrel monkey. III. Variations among units in their discharge properties. J Neurophysiol 34 676-684.

Hamid MA (1990) Clinical value of sinusoidal harmonic acceleration test results; site of lesion and side of lesion. Neurologic Clinics 8 (2) 287-295.

Hamming RW (1983) Digital filters. Prentice-Hall, Englewood Cliffs, New Yersey. ISBN 0-13-212506-4. 
Hine T, Thorn F (1987) Compensatory eye movements during active head rotation for near targets: effects of imagination, rapid head oscillation and vergence. Vision Research 27 1639-1657.

Hodgson RM, Bailey DG, Naylor MJ, Ng ALM, McNeill SJ (1985) Properties, implementations and applications of rank filters. Image Vision Comp 3 (1) 3-14.

Honrubia V, Downey WL, Mitchell DP, Ward PH (1968) Experimental studies on optokinetic nystagmus. II. Normal humans. Acta Otolaryngol 65 441-448.

Honrubia V, Katz RD, Strelioff D, Ward PH (1971) Computer analysis of induced vestibular nystagmus rotary stimulation of normal cats. Ann Otol-RhinolLaryngol Suppl 382 7-24.

Honrubia V, Jenkins HA, Baloh RW, Lau CGY (1982) Evaluation of rotatory vestibular tests in peripheral labyrinthine lesions. In Clinical approaches to the patient with dizziness. (ed Honrubia V, Brazier MAB) Academic Press, New York, 57-80.

Honrubia V, Khalili R, Baloh RW (1992) Optokinetic and vestibular interactions with smooth pursuit (psychophysical responses). Ann New York Acad Sci 656739 746, (also in Acta Otolaryngol (Stockh) 112 163-169).

Howard IP (1986) chapter 18 The perception of posture, self motion, and the visual vertical in: Handbook of perception and human performance Volume I (sensory processes and perception) John Wiley and Sons, New York, ISBN 0-47188544-4 (two-volume set: ISBN 0-471-82956-0)

Huebner WP, Leigh RJ, Seidman SH, Thomas CW, Billian C, DiScenna AD, Del'Osso LF (1992) Experimental tests of a superposition hypothesis to explain the relationship between the vestibular reflex and smooth pursuit during horizontal combined eye-head tracking in humans. I Neurophysiol 68 (5) 1775 1792.

Hydén D, Istl YE, Schwarz DWF (1982) Human visuovestibular interaction as a basis for quantitative clinical diagnostics. Acta Otolanngol (Stockh) 94 53-60.

Juhola M, Jäntti V, Pyykkö, Magnusson M, Schalén L, Åkesson M (1985) Detection of saccadic eye movements using a non-recursive adaptive digita] filter. Comput Methods Programs Biomed 21 81-88. 
Kasteel-van Linge A, Maas AJJ (1990) Quantification of visuo-vestibular interaction up to $5.0 \mathrm{~Hz}$ in normal subjects. Acta Otolaryngol (Stockh) 110 18-24.

Keller EL (1978) Gain of the vestibulo-ocular reflex in monkey at high rotational frequencies. Vision Res 18 311-315.

Larsby B, Hydén D, Ödkvist LM (1984) Gain and phase characteristics of compensatory eye movements in light and darkness. Acta Otolaryngol (Stockh) 97223 232.

Laurutis VP, Robinson DA (1986) The vestibulo-ocular reflex during human saccadic eye movements. I Physiol (London) 373 209-233.

Leigh RJ, Zee DS (1983) The neurology of eye movement. FA Davis Company, Philadelphia, ISBN 0-8036-5524-X.

Louie AW, Kimm J (1976) The response of VIIIth nerve fibres to horizontal sinusoidal oscillation in the alert monkey. Exp Brain Res 24 447-457.

Magenes G, Schmid R, Ventre J (1990) The role of smooth pursuit in suppression of post-rotational nystagmus. Aviar Space Environ Med 61 (2) 117-124.

Maki EB (1986) Interpretation of the coherence function when using pseudorandom inputs to identify nonlinear systems. IEEE Trans Biomed Eng BME-33(8) 775-779.

Maki EB (1988) Addendum to "Interpretation of the coherence function when using pseudo-random inputs to identify noninear systems". IEEE Trans Biomed Eng BME-35(6) 279.

McKinley PA, Peterson BW (1985) Voluntary modulation of the vestibulo-ocular reflex in humans and its relation to smooth pursuit. Exp Brain Res 60 454-464.

Miles FA, Busettini C (1992) Ocular compensation for self-motion (visual mechanisms). Ann New York Acad Sci 656 220-232.

Mishkin S, Melvill Jones G (1966) Predominant direction of gaze during slow head rotation. Aerospace Medicine 37 897-900. 
Mittelstaed H (1992) Somatic versus vestibular gravity reception in man. Amn New York Acad Sci 656 124-139

Moller C, White V, Ödkvist LM (1990) Plasticity of compensatory eye movements in rotatory tests. II The effect of voluntary, visual, imaginary, auditory and proprioceptive mechanisms. Acta Otolaryngol (Stockh) 109 168-178.

Norušis MJ (1986) SSPSS/PC+ for the IBM PC/XT/AT (Advanced Statistics) SPSS inc., Chicago, ISBN 0-918469-15-5.

O'Leary DP, Davis LL, Kitsigianis GA (1988) Analysis of vestibulo-ocular reflex using sweep frequency active head movements. Adv Otol Rhinol Laryngol 41 179183.

O'Leary DP, Davis LL (1990) Vestibular autorotation testing of Meniere's disease. Oto-Lanyngol Head Neck Surg 103 66-71.

Ödkvist LM, Thell J, Larsby B (1988) A comparison between smooth pursuit and visual suppression. Adv Oto-Rhino-Laryng 41 109-115.

Owen Black F, Wall III C (1984) Electronystagmographical Computerization in: Otoneurology (ed. WJ Oosterveld) John Wiley and Sons, New York, ISBN 0-47190441-4 1-22.

Precht W, Dieringer N (1985) Neuronal events paralleling functional recovery (compensation) following peripheral vestibular lesions in: Reviews of oculomotor research (volume 1) Adaptive mechanisms in gaze control (editors: Berthoz A, Melvill Jones G) Elsevier, Amsterdam, ISBN 0-444-80483-8 251-268.

Press WH, Flannery BP, Teukolsky SA, Vetterling WT (1988) Numerical recipes in $C$ (the art of scientific computing). Cambridge University Press, Cambridge, ISBN 0-52135465-X.

Reason JT (1970) Motion sickness: a special case of sensory rearangement. $A d v$ Sci 26 386-393.

Reason JT (1978) Motion sickness adaptation: a neural mismatch model. J Roy Soc Med 71 819-829. 
Royston P, Matthews JNS (1991) Estimation of reference ranges from normal samples. Statistics in Medicine $10691-695$.

Sackett DL, Haynes RB, Tugwell P (1985) Clinical epidemiology (a basic science for clinical medicine). Little, Brown and Co, Boston, ISBN 0-316-76595-3.

Shimazu H, Precht W (1966) Inhibition of central vestibular neurons from the contralateral labyrinth and its mediating pathway. J Nevurophysiol 29 467-492.

Sokal RR, Rohlf FJ (1981) Biometry (the principles and practice of statistics in biological research). WH Freeman and Company, New York, ISBN 0-7167-1254-7.

Stark L, Vossius G, Young LR (1962) Predictive control of eye tracking movements. IEEE Trans Human Factors Electron HFE-3 52-57.

Steinbach M (1976) Pursuing the perceptual rather than the retinal stimulus. Vision Research 16 1371-1376.

Tukey JW (1977) Exploratory data analysis. Addison-Wesley, Reading, Massachusets.

Wall III C, Black FO, Hunt E (1984) Effects of age, sex and stimulus parameters upon vestibulo-ocular responses to sinusoidal rotation. Acta Otolaryngol (Stockh) $98 \quad 270-278$.

Welch PD (1978) in Modem spectrum analysis (ed. Childer DG) IEEE Press, New York, cited in Press et al. (1988).

Westheimer G (1986) chapter 4 The eye as an optical instrument in: Handbook of perception and human performance Volume I (sensory processes and perception) John Wiley and Sons, New York, ISBN 0-471-88544-4 (two-volume set: ISBN 0471-82956-0)

Wijvekate ML (1972) Verklarende statistiek. Aula/Het Spectrum, Utrecht. (In Dutch).

Wilson VJ, Melvill Jones G (1979) Mammalian vestibular physiology. Plenum Press, New York, ISBN 0-306-40134-7. 
Wolfe JW, Engelken EJ, Olson JE (1982) Low-frequency harmonic acceleration in the evaluation of patients with peripheral labyrinthine disorders in: Nystagmus and vertigo (clinical approaches to the patient with dizziness) (editors: Honrubia V, Brazier MA) Academic Press, New York, ISBN 0-12-355080-7 95-105.

Yasui S, Young L (1975) Perceived visual motion as effective stimulus to pursuit eye movement system. Science 190 906-908.

Young LR, Henn VS (1974) Selective habituation of vestibular nystagmus by visual stimulation. Acta Otolaryng 77 159-166.

Yu M, Wu L, Wu D (1990) Mode] parameters of the smooth pursuit eye movement system with electrooculogram. Doc Ophthalmologica 76 37-46. 


\section{Samenvatting}

Een vestibulaire functietest (VVIT genaamd: Visuo-Vestibulaire Interactie Test) wordt gepresenteerd, waarbij een visueel doel asynchroon met een pseudo-random oscillerende draaistoel beweegt.

De resultaten van deze test laten een betere classificatie toe van de hier onderzochte patiënten dan de resultaten van de gebruikte standaardtesten (enkelvoudige sinusoidale oscillatie in het donker, calorisatie). De specificiteit met betrekking tot enkel normalen, is 1.00 voor de zes gebruikte controlepersonen, en de sensitiviteit is hoger dan dat van de gebruikte standaardtesten voor Meniere-patiënten, centraal slecht gecompenseerde perifeer-vestibulaire patiënten, whiplash-patiënten, en patiënten met benigne paroxysmale positioneringsduizeligheid (BPPD), maar niet voor centraal goed gecompenseerde perifeer-vestibulaire patiënten en patiënten voor wie de oorzaak van hun klachten onbekend is. Whiplash-patiènten, Meniere-patiënten en BPPD-patiënten zijn de groepen voor wie de VVIT de beste combinaties heeft van sensitiviteit en specificiteit zowel ten opzichte van andere patiëntengroepen als ten opzichte van controles.

Zes normalen en 64 patiënten zijn onderzocht, en van de laatsten bleven er 51 over voor analyse.

Aanzienlijke moeite is gestoken in het vinden en corrigeren van meetfouten, hetgeen tamelijk gedetailleerd wordt besproken.

Signaalverwerking omvatte het gebruik van rangorde-filters om snelle componenten te verwijderen en om oogknipper-artefacten af te zwakken. Het mediaanfilter werd veralgemeniseerd tot een "variabel percentielfilter" om nystagmoide oogbewegingssignalen te kunnen verwerken. Het werd tevens toegepast op stimulus-geordende (en daardoor tijdgeklutste) signalen om met lineaire regressie toch van pseudo-random signalen de versterking te kunnen bepalen zonder toevlucht te hoeven nemen tot frequentie-analyse, waardoor het mogelijk was om de versterking voor links- en rechtsgerichte bewegingen te vergelijken.

Voor signaalanalyse werd echter voornamelijk frequentie-analyse gebruikt. Versterking, faseverschuiving en coherentie werden in het frequentiedomein berekent.

De resultaten werden statistisch geanalyseerd met verschillende vormen van multivariate variantie-analyse (MANOVA's), en door de sensitiviteit en specificiteit te berekenen met gebruik van zowel standaard als zuivere referentiebereiken. 


\section{Dankwoord / Acknowledgements}

Mijn promotor, Prof.Em. dr. E.H.M.A. Marres wil ik bedanken voor het vertrouwen dat hij in me heeft gesteld, voor zijn zorg voor de mogelijkheden tot het doen van wetenschappelijk onderzoek en voor de volle vrijheid die hij me heeft gelaten.

Co-promotor, dr. H. Kingma, beste Herman, de wijze waarop jij enthousiasme om nieuwe onderzoekswegen in te gaan paart met een kritische instelling ten opzichte van methodiek en verwerking, dwingt alleen al bewondering af. Maar bovendien combineer je dat met een fijne stijl van management en met commercieel inzicht. Deze vier zaken hebben de stormachtige ontwikkeling van de vestibulaire afdeling binnen de vakgroep Keel-, Neus- en Oorheelkunde te Maastricht mogelijk gemaakt en $i k$ ben blij dat het hier beschreven onderzoek daar een onderdeel van heeft mogen vormen. Temeer daar dit alles plaatsvond in een tijd van toenemende lastenafwenteling ("bezuiniging").

Verder vormen de door jou gestelde diagnoses van de vestibulaire patiênten één van de pilaren van deze studie.

Dr. G.R. Barnes, the patience with which you explained aspects of visuo-vestibular interaction during a break at the Durham conference, and with which you answered all my fax-sent questions, made it a pleasant task to correct parts of the draft version of this thesis.

Dr. J. Patijn, beste Jaap, we danken je voor het vertrouwen waarmee je whiplashpatiënten van de Stichting Manueel Centrum Eindhoven doorverwees naar de vestibulaire afdeling voor visuo-vestibulaire diagnostiek en voor het stimulerende brainstormen over functiestoornissen.

Dr. P.L.M. Huygen, beste Patrick, jouw gedegen beoordeling van en kritische kanttekeningen bij het ontwerp van m'n voordracht voor de Báràny society in Praag hebben zeker ook doorgewerkt in dit proefschrift.

Dr. H. Schouten, beste Huub, de snelheid waarmee je direct antwoord gaf op vragen over statistiek die op willekeurige tijdstippen op de dag telefonisch werden gesteld, heeft de vaart gehouden in de verwerking van de resultaten.

Marlies Dolmans, jouw flexibiliteit maakte het mogelijk dat mijn onderzoek verweven werd met de patiëntenzorg, waar je als ENG-assistente mee belast bent. En je aanhoudende interesse dwong me tot het helder proberen te verwoorden van de doelstellingen, resultaten en conclusies. 
Ing. Iwan de Jong en ing. Ron Jonge, jullie ondersteuning op het gebied van software en hardware schiep een experimenteeromgeving die maar al te gemakkelijk als vanzelfsprekend kan worden ervaren.

Gerard Houben en Guido Zonneveld, het gezamenlijk bekijken van elkaars problemen op het gebied van signaalverwerking, tekstverwerking en grafische weergave maakte het altijd aanwezige conflict tussen wat de wetware wenst en wat de hard- en software toelaten, tot een bron van vermaak en voldoening.

Mirjam Bonink en Ellis van Lieshout, jullie inzet bij de eerste serie metingen maakte het mogelijk dat we al snel idee kregen hoe zinvolle meetresultaten konden worden verkregen. Jullie stages vielen in de periode van uitproberen van technieken. Ik besef hoe sneu het is dat we hebben moeten concluderen dat de toen gebruikte methode onjuist was, waardoor er hier in dit proefschrift niets van terug te vinden is. Maar toch is die periode waardevol geweest omdat die ons de gelegenheid bood om de keuze van een meettechniek te baseren op ervaring.

Marianne Simons, ook jouw stage betrof het minder glorieuze deel van wetenschapsbeoefening, namelijk het opsporen van fouten bij het meten en bij de data-analyse. Meer produkten van onze samenwerking zijn belangrijk geweest dan die paar jouw bekende grafieken die hier zijn opgenomen.

Frank van Aelen, jij hebt laten zien dat in een later stadium van onderzoek zelfs een korte stage tot een goed afgerond werkstuk kan leiden. Jouw programmaatje om de door Marianne handmatig uitgevoerde analyse te automatiseren vergemakkelijkte vervolgonderzoek aanmerkelijk. Het nut ervan moge blijken uit het feit dat een andere groep het nu ook gebruikt.

Alle niet-genoemde leden van de KNO-vakgroep, met wie het samen werken en samenwerken een genoegen was.

De patiënten, zonder wie dit onderzoek niet mogelijk zou zijn geweest.

Traditiegetrouw wordt in een hoofdstuk als dit de partner het laatst vermeld, maar voor mij komt ze op de eerste plaats. Lieve Lin, jouw zelfopoffering en jouw toewijding hebben the het mogelijk gemaakt om zo lang geobsedeerd bezig te zijn geweest. Terima kasih, sayang saya! Dyan en Sander, bedankt voor jullie geduld; papa zall voortaan vaker met jullie spelen. 


\section{Curriculum Vitae}

De schrijver van dit proefschrift werd geboren op 4 juni 1955 te Utrecht. Na het behalen van de diploma's voor ULO-B (1971), HAVO (1973) en Atheneum (1974) werd een studie Biologie aan de Rijksuniversiteit Utrecht begonnen. In 1978 werd het kandidaatsexamen behaald voor de richting Moleculaire Celbiologie (judicium: "met genoegen"). Aansluitend werd de doctoraalfase doorlopen, waarbij de belangstelling behalve naar de bij de gekozen onderwerpen (virus mRNA, kernmorfometrie, beeldinformatica) behorende cursussen en werkzaamheden ook uitging naar andere gebieden. Aanvankelijk in de richting van Microbiologie en Stralingshygiene, maar later meer naar de Mathematische Biologie. Enige op free-lance basis geschreven populairwetenschappelijke artikeltjes werden gepubliceerd op de wetenschapspagina van het dagblad Trouw. Op 27 mei 1987 werd deze fase afgesloten met het behalen wan het doctoraaldiploma (oude stijl; judicium: "met genoegen").

Het AIO-contract van 1 april 1988 tot 1 april 1992 bij de Rijksuniversiteit Limburg werd vanwege de verhuizing van het Academisch Ziekenhuis Maastricht, waar hij bij de Vestibulaire afdeling van de vakgroep Keel-, Neus- en Oorheelkunde gestationeerd was, verlengd tot 1 jamuari 1993. Sindsdien is hij werkzoekend; gedurende de eerste 9 maanden is dit proefschrift afgerond en is op free-lance basis statistische ondersteuning gegeven aan de Audiologische afdeling van dezelfde vakgroep.

Hij is gehuwd sinds 1990 en heeft een dochter en een zoon. 


\section{Errata}

p. 12: fig. 2-4: delaying block $\mathrm{e}^{\text {st }}$ should follow, not lead, gain block $\mathrm{K}_{\mathrm{v}}$.

p. 25: fig. 3-12: "0.42" in legend should be "0.41".

p. 32: fig. 4-2: figure has disappeared, please insert the one given below.

p. 48: "Thorne" should be "Thorn"

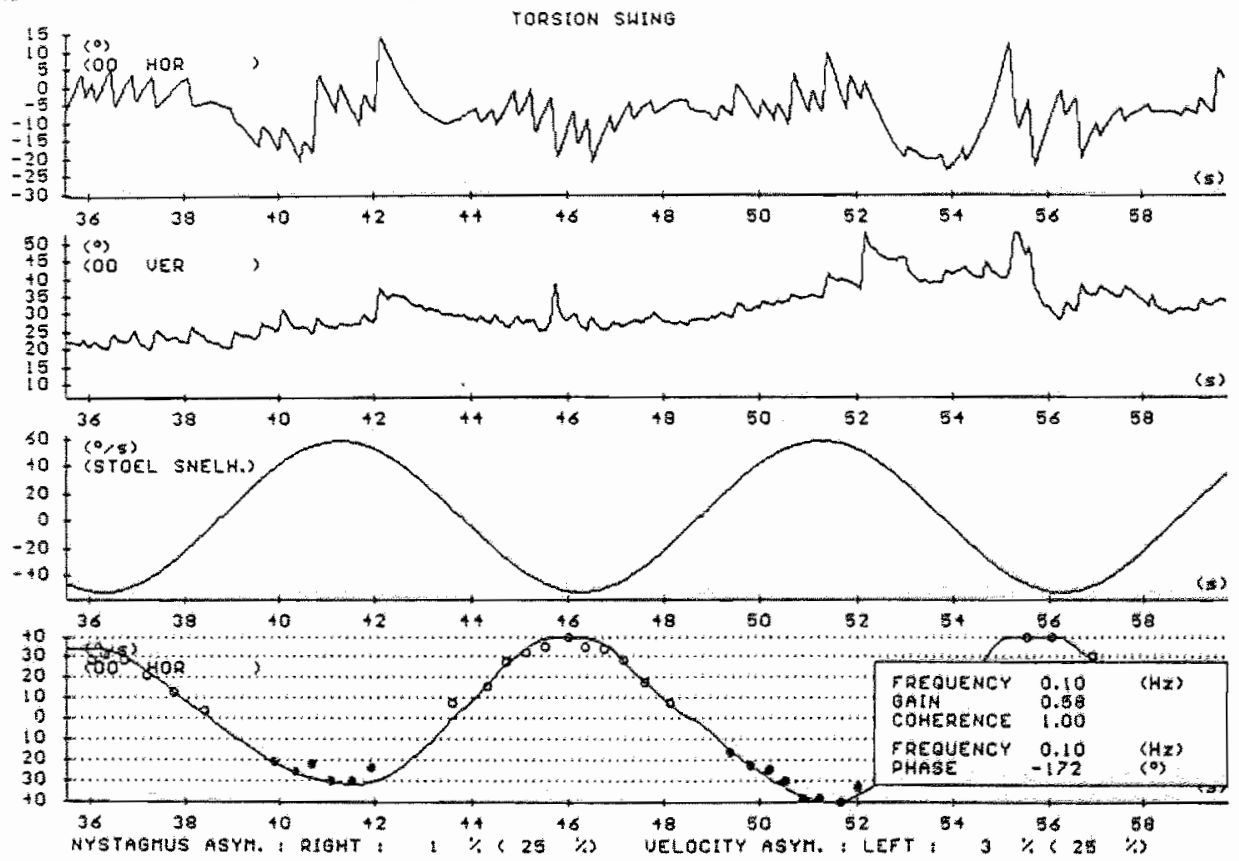

Fig. 4-2. 